RPP-6251

Revision 0

\title{
Dynamic Object Oriented Requirements System (DOORS), System Test Plan
}

Prepared for the U.S. Department of Energy Assistant Secretary for Environmental Management

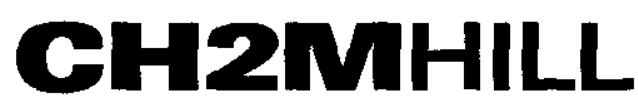

Hanford Group, Inc.

Richland, Washington

Contractor for the U.S. Department of Energy

Office of River Protection under Contract DE-AC06-99RL14047

Approved for Public Release 


\section{INFORMATION CLEARANCE FORM}

\begin{tabular}{ll|l}
\hline \multicolumn{2}{|c|}{ A. Information Category } & B. Document Number RPP-6251 \\
$\square$ Abstract & $\square$ Journal Article & C. Title \\
$\square$ Summary & $\square$ Internet & Dynamic Object Oriented Requirements System (DOORS), System Test \\
$\square$ Visual Aid & $\square$ Software & Plan \\
$\square$ Full Paper & $\square$ Report & \\
$\square$ Other & \\
\cline { 3 - 3 } & & D. Internet Address \\
\hline
\end{tabular}

E. Required Information

1. Is,document potentially Classified? $\triangle$ No $\square$ Yes (MANDATORY)

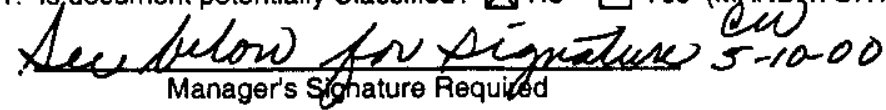

a. New or Novel (Patentable) Subject Matter? $\quad{ }_{\text {No }} \square$ Yes

If "Yes", Disclosure No.:

b. Information Received in Confidence, Such as Proprietary and/or Inventions?

If Yes $\square$ No $\square$ Yes Classified ADC Signature Required

$\square$ No $\square$ Yes If "Yes", Aftix Appropriate Legends/Notices.

c. Copyrights? $\square$ No $\square$ Yes If "Yes", Attach Permission.

2. Internal Review Required? $\square$ No $\square$ Yes If Yes, Document Signatures Below

d. Trademarks? $\square$ No $\bigotimes$ Yes If "Yes", Identify in Document.

Counsel

5. Is information requiring submission to OSTI? $\square$ No $\square$ Yes

Program

If Yes UCand $B \& R-$

3. References in the Information are Applied Technology $\bigotimes_{\text {No }} \square$ Yes 6. Release Level? \ Public $\square$ Limited

Export Controlled Information 凶No $\square$ Yes

7. Charge Code $112003 / \mathrm{AOONO} H M L M O 441$

F. Complete for a Journal Article

1. Title of Journal .

G. Complete for a Presentation

1. Title for Conference or Meeting

2. Group Sponsoring

3. Date of Conference

4. City/State

5. Will Information be Published in Proceedings? $\square$ No $\square$ Yes

6. Will Material be Handed Out? $\square$ No $\square$ Yes

H. Author/Requestor

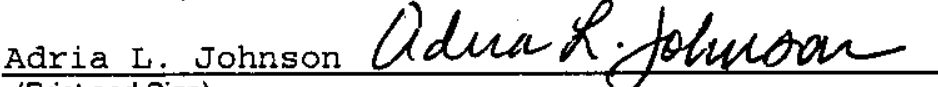
(Print and Sign)

Responsible Manager

Ronald L. Nelson (Print and Sign)
I. Reviewers
Yes Print
General Counsel
$\bigotimes \quad$ Steven D. Brumley

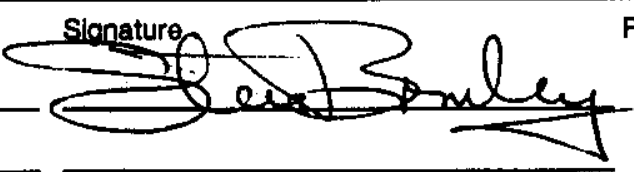
Office of External Affairs
DOEsTit CRP
$\triangle$ Jerlinda D. (Linda) Banks
Other

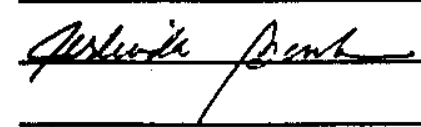
Y Y N

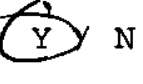
$\mathrm{Y} / \mathrm{N}$
Y.DN
$\mathrm{Y} / \mathrm{N}$
$Y / N$
Other

Public $Y / N$ (If $N$, complete J)

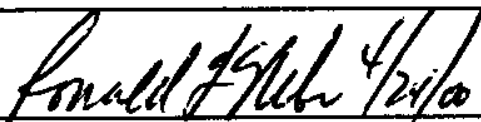

J. If Information Includes Sensitive Information and is not to be released to the Public indicate category below.

$\square$ Applied Technology $\square$ Protected CRADA

$\square$ Personal/Private $\square$ Export Controlled

$\square$ Proprietary $\square$ Procurement-Sensitive

$\square$ Business-Sensitive $\square$ Patentable

$\square$ Predecisional $\square$ Other (Specify)

$\square$ UCNI

K. If Additional Comments, Please Attach Separate Sheet 
RPP-6251

Revision 0

\section{Dynamic Object Oriented Requirements System (DOORS), System Test Plan}

A. L. Johnson
Lockheed Martin Services, Incorporated

Date Published

April 2000

Prepared for the U.S. Department of Energy

Assistant Secretary for Environmental Management

\section{CH2MHILL \\ Hanford Group, Inc.}

P. O. Box 1500

Richland, Washington

Contractor for the U.S. Department of Energy

Office of River Protection under Contract DE-AC06-99RL14047 
LEGAL DISCLADIER

This report was prepared as an acocurt of work sponsored by an agency of the United States Governmert. Neither the United States Government nor any agexcy thereof, nor any of their employees, nor any of their contractors, subcortrations or their employees, makes any warrarty, express or implied, or assumes any legat liability or responsibility for the accuracy, completeness, of any third party's use or the results of such use of any information, apparatus, product, or process disclosed, or represents that its use would not infinge privately owned dights. Reference herein to any specific commercial product, process, or service by trade name, trademark, manufacturer, oc otherwise, does not necessanily constiture $\alpha$ imply its endorsement, recommendation, $\alpha$ favoring by the United States Governmere or any agency thereof or its coneractors or subcontractors. The view/s and opinions of authors expressed herein do nox necessarily state or reflect those of the United States Government or any agency thereot.

This report has been reproduced from the beat available copy.

Pitiod in to Uaited States of Aberien

DISCLM-2.CHP (1-91) 


\section{RELEASE AUTHORIZATION}

Document Number:

Document Title:
RPP-6251, Rev. 0

Dynamic Object Oriented Requirements System (DOORS), System Test Plan

This document, reviewed in accordance with DOE Order 241.1, "Scientific and Technical Information Management," and DOE G 241.1-1, "Guide to the Management of Scientific and Technical Information," does not contain classified or sensitive unclassified information and is:

\section{APPROVED FOR PUBLIC RELEASE}

Christain

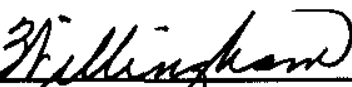

C. Willingham

$5 / 10 / 00$

Lockheed Martin Services, Inc.

Document Control//nformation Clearance

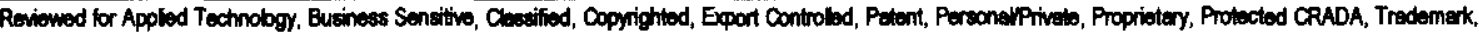
Unclassified Controlled Nuclear Informetion.

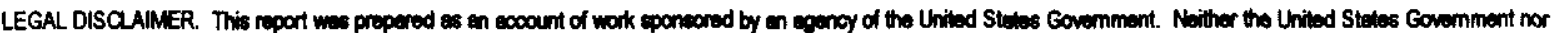
any agency thereof, not any of their employees, nor any of their contractors, scbcontructors or their employees, mekes any werrenty, express or inpled, or asaumes any begal

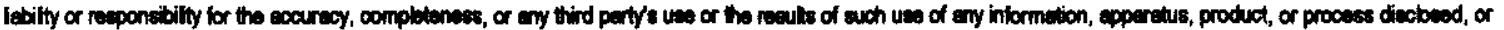

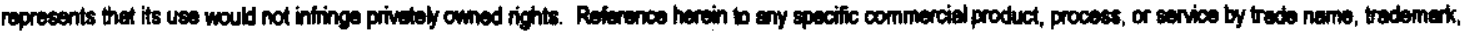
menufacturer, or otherwise, does not necesserily constitute or imply its endorsement, recommendetion, or favoring by the Unitad States Covemment or any agency thereof or its contrectors or subcontractors. The views end opinions of authors expressed heroin do not necesserily state or reflect those of the Unitod States Govemment or any agency thereof. This report hes been reprocuced from the best avaibble copy. Pinted in the United Stetes of America. 
RPP-6251 REV. 0

\section{TEST PLAN APPROVALS}

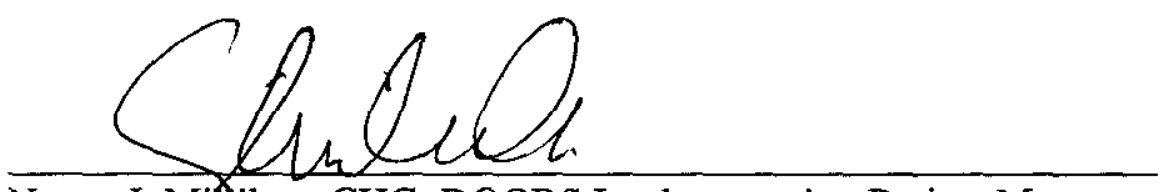

Nancy J. Milliken, CHG, DOORS Implementation Project Manager

Aduin L. phrson

Adria L. Johnson, LMSI, SD\&I, DOORS Project Lead

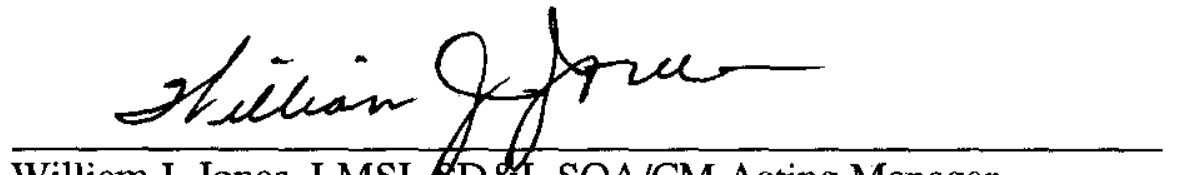

William J. Jones, LMSI, OD\&I, SQA/CM Acting Manager
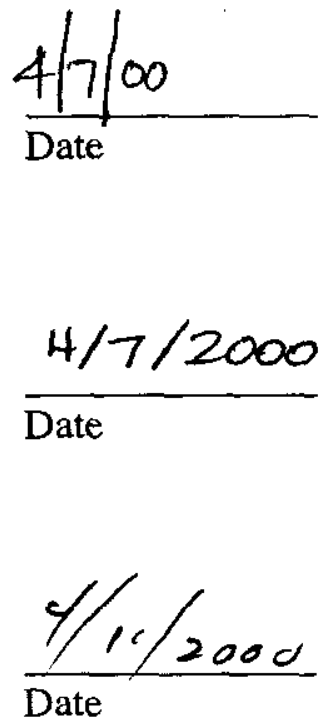


\section{TABLE OF CONTENTS}

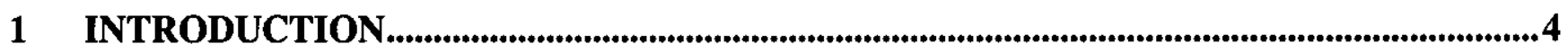

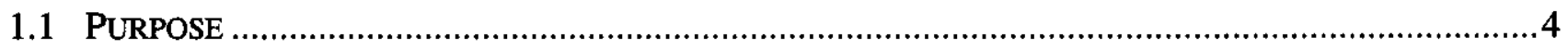

1.2 SCOPE . . . .

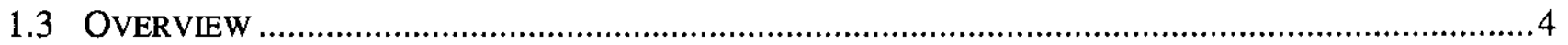

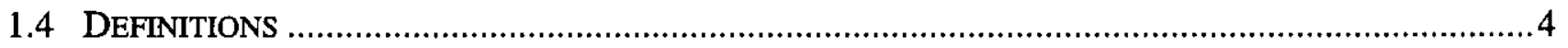

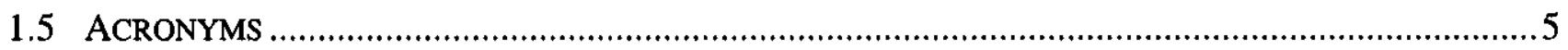

2 TEST PLAN

2.1 TEST ITEMS

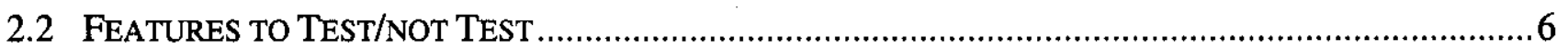

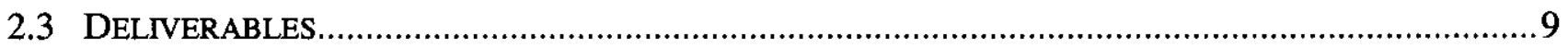

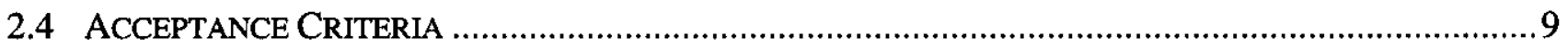

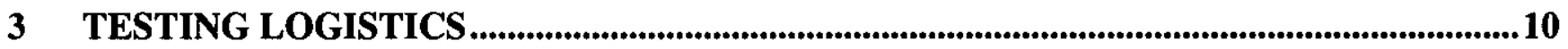

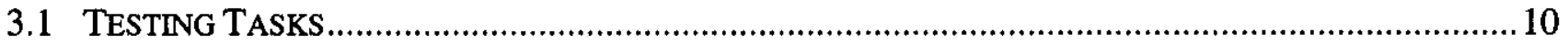

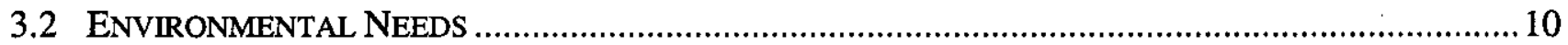

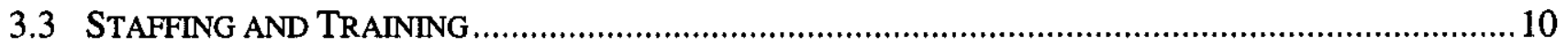

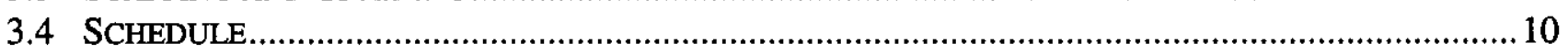

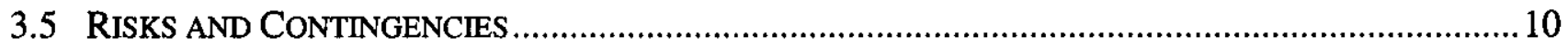

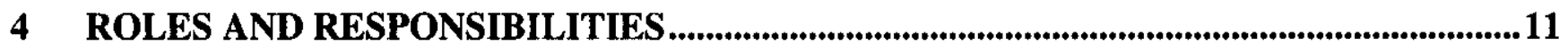

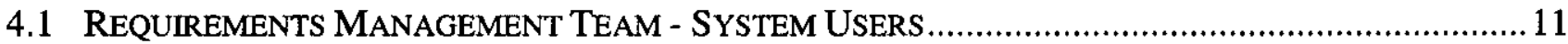

4.2 LOCKHEED MARTIN SERVICES, INC. - DOORS TECHNICAL TEAM..........................................11

4.3 PROJECT MANAGEMENT \& LEAD RESPONSIBILITIES ............................................................. 11

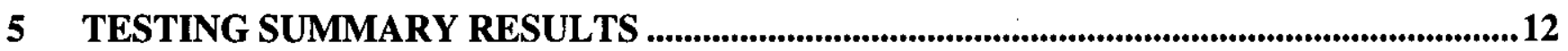

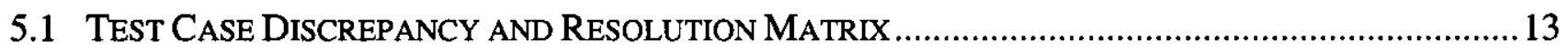

6 CUSTOMER ACCEPTANCE..................................................................................................15

7 APPENDIX A - REQUIREMENTS TRACEABILITY MATRIX …….......................................16

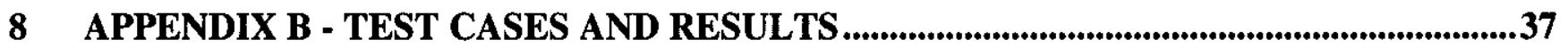

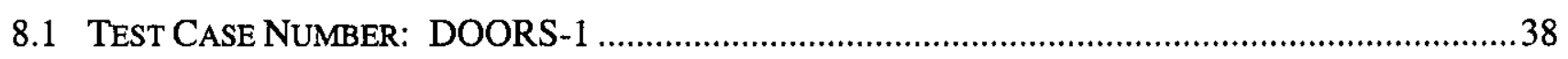

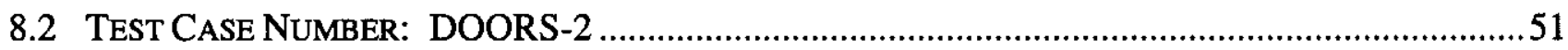

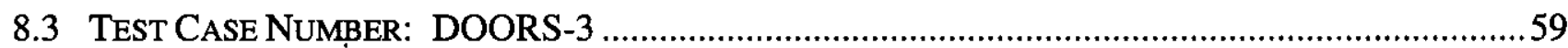

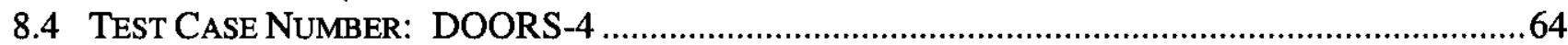

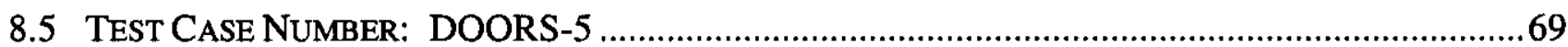

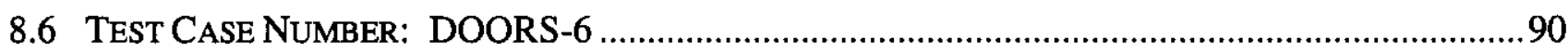

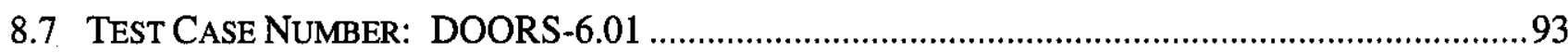

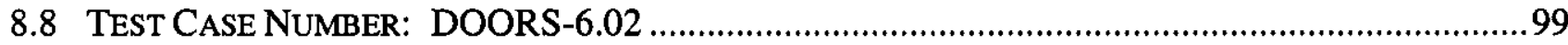

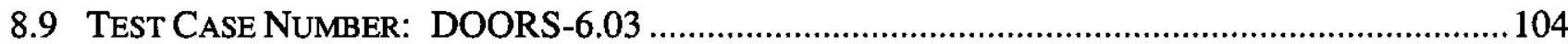

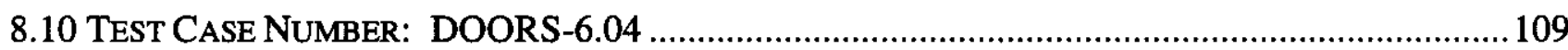

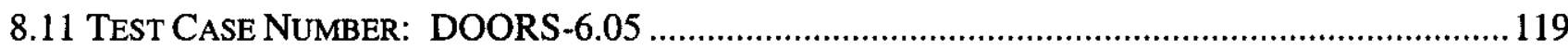

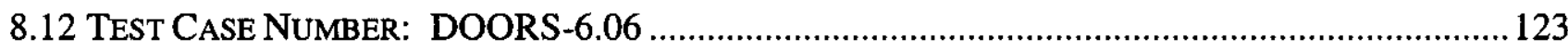




\section{INTRODUCTION}

\subsection{Purpose}

The U. S. Department of Energy, Office of River Protection (ORP) will use the Dynamic Object Oriented Requirements System (DOORS) as a tool to assist in identifying, capturing, and maintaining the necessary and sufficient set of requirements for accomplishing the ORP mission. By managing requirements as one integrated set, the ORP will be able to carry out its mission more efficiently and effectively.

DOORS is a Commercial-Off-The-Shelf (COTS) requirements management tool. The tool has not been customized for the use of the PIO, at this time.

\subsection{Scope}

This test plan will be used as the primary medium for communicating acceptance testing criteria. It will include the testing scenarios created to test the requirements listed in RPP-5865, Office of River Protection, Project Integration Office, Requirements Management System, Requirements Specification. This test plan will also address other system testing needs, as determined by the DOORS technical team.

This test plan is limited to the use of the DOORS tool, no data will be verified or tested. Testing will be performed to demonstrate the high level ability for the tool to meet the requirements. It does not apply to manual requirements management business processes defined by the user.

\subsection{Overview}

The ORP Project Integration Office (PIO) has been tasked with the identification, capture, and maintenance of requirements imposed upon the River Protection Project (RPP). The PIO

Requirements Management team has identified an initial set of source documents that will be the first to be entered into DOORS. User defined attributes will be assigned to each of the objects created within the source document modules.

\subsection{Definitions}

Project - The highest level of distinction within DOORS. Each project is a unique database that has its own independent set of users who can access information contained within it.

Modules - All information contained in DOORS is stored in Modules. DOORS module classes include formal, descriptive, and link.

Formal Module: A DOORS module containing structured sets of similar information.

Descriptive Module: A DOORS module containing unstructured source information.

Link Module: A DOORS module containing and managing relationships between other data elements. 
Objects - A DOORS Object is the basic building block of every DOORS module, and typically contains a requirement. Objects can be created deleted, edited, copied, and moved. Each object has a unique identifier that cannot be changed.

Attributes - All modules, objects, and links possess Attributes, both system defined and user defined. System defined attributes include a unique identifier, creation date and when the object was last modified. Any number of user defined attributes can be created.

History - All changes made to modules, objects, and attributes are recorded in module history. The module history records who made the change, when, and what the change was.

\subsection{Acronyms}

$\begin{array}{ll}\text { BNFL } & \text { British Nuclear Fuels, Limited } \\ \text { CHG } & \text { CH2M Hill Hanford Group } \\ \text { CIO } & \text { Chief Information Officer } \\ \text { COTS } & \text { Commercial Off-The-Shelf } \\ \text { CP } & \text { Change Proposal } \\ \text { DOE } & \text { U. S. Department of Energy } \\ \text { DOORS } & \text { Dynamic Object Oriented Requirements System } \\ \text { DXL } & \text { DOORS Extension Language } \\ \text { HLAN } & \text { Hanford Local Area Network } \\ \text { LMSI } & \text { Lockheed Martin Services, Incorporated } \\ \text { NT } & \text { New Technology } \\ \text { ORP } & \text { U. S. Department of Energy, Office of River Protection } \\ \text { OS } & \text { Operating System } \\ \text { PHMC } & \text { Project Hanford Management Contract } \\ \text { PIO } & \text { Project Integration Office } \\ \text { PNNL } & \text { Pacific Northwest National Laboratory } \\ \text { PRRB } & \text { Production Readiness Review Board } \\ \text { QSS } & \text { Quality Systems and Software, Incorporated } \\ \text { RL } & \text { U. S. Department of Energy, Richland Operations Office } \\ \text { RPP } & \text { River Protection Project } \\ \text { SQL } & \text { Standard Query Language }\end{array}$


RPP-6251 REV. 0

\section{TEST PLAN}

\subsection{Test Items}

A variation of the Requirements Traceability Matrix provides the description for the testing of customer requirements. Refer to Appendix A. The matrix was derived from RPP-5865, Office of River Protection, Project Integration Office, Requirements Management System, Requirements Specification.

Additional testing will be performed on infrastructure, network, and server test cases, as determined by the LMSI technical team.

Test cases may provide explicit test steps for testing a requirement but where such details are not provided it is expected that a knowledgeable tester will use his or her knowledge of DOORS and of the requirements to appropriately test a requirement.

The LMSI DOORS technical team will perform all test cases. The DOORS users will be invited to participate in the testing. During user testing, members of the LMSI technical team will be available for providing additional information about interpreting the makeup and intent of specific test cases.

\subsection{Features to Test/not Test}

Every requirement listed in the Requirements Traceability Matrix was evaluated for testing criteria. DOORS is a COTS product and not all of the available functionality will be used by the PIO. This test plan focuses on the functionality needed to fulfill the user-defined requirements. Requirements that will be tested are sorted into related groups and will be tested in test cases of the following areas:

- Change Proposal System

- User Access

- Filtering, Sorting, and Reporting

- Project, Module, and Attribute Creation and Manipulation

- DOORSNet

The following requirements do not refer to the use of the tool or do not contain enough specific information to warrant testing:

\begin{tabular}{|l|l|l|}
\hline No. & \multicolumn{1}{|c|}{ Requirement } & \multicolumn{1}{c|}{ Resolution } \\
\hline 5 & $\begin{array}{l}\text { Their querying and reporting will be } \\
\text { infrequent. Any electronic tool must be } \\
\text { intuitive in order to keep support costs to a } \\
\text { minimum. }\end{array}$ & $\begin{array}{l}\text { General use of DOORS will be via } \\
\text { DOORSNet, which requires minimal } \\
\text { introduction for use. }\end{array}$ \\
\hline 10 & $\begin{array}{l}\text { Will identify potential new requirements and } \\
\text { make recommendations to ORP } \\
\text { management and a requirements change } \\
\text { board. }\end{array}$ & $\begin{array}{l}\text { Identification of new requirements is } \\
\text { developed through human interface. }\end{array}$ \\
\hline 11 & $\begin{array}{l}\text { Will make recommendations to ORP } \\
\text { management and a requirements change } \\
\text { board. }\end{array}$ & $\begin{array}{l}\text { Recommendations are made through a } \\
\text { human interface. }\end{array}$ \\
\hline
\end{tabular}




\begin{tabular}{|c|c|c|}
\hline No. & Requirement & Resolution \\
\hline 16 & $\begin{array}{l}\text { Will identify potential new interface } \\
\text { requirements and make recommendations to } \\
\text { ORP management and a requirements } \\
\text { change board. }\end{array}$ & $\begin{array}{l}\text { Tdentification of new requirements is } \\
\text { developed through human interface. }\end{array}$ \\
\hline 17 & $\begin{array}{l}\text { Will make recommendations to ORP } \\
\text { management and a requirements change } \\
\text { board. }\end{array}$ & $\begin{array}{l}\text { Recommendations are made through human } \\
\text { interface. }\end{array}$ \\
\hline 23 & $\begin{array}{l}\text { They will be very infrequent users of the } \\
\text { ORP-PIO Requirements management } \\
\text { Database. }\end{array}$ & $\begin{array}{l}\text { General use of DOORS will be via } \\
\text { DOORSNet, which requires minimal } \\
\text { introduction for use. }\end{array}$ \\
\hline 24 & No requirements defined. & $\mathrm{N} / \mathrm{A}$ \\
\hline 25 & $\begin{array}{l}\text { Will also oversee the change control process } \\
\text { for requirements. }\end{array}$ & $\begin{array}{l}\text { Oversight is performed through a human } \\
\text { interface. }\end{array}$ \\
\hline 27 & $\begin{array}{l}\text { Will sometimes query the requirements } \\
\text { database. }\end{array}$ & $\begin{array}{l}\text { General use of DOORS will be via } \\
\text { DOORSNet, which requires minimal } \\
\text { introduction for use. }\end{array}$ \\
\hline 32 & $\begin{array}{l}\text { The ORP Requirements Management } \\
\text { System must be able to contain all } \\
\text { requirements as a single integrated set. }\end{array}$ & $\begin{array}{l}\text { The ORP Requirements will be located in a } \\
\text { single project. }\end{array}$ \\
\hline 33 & $\begin{array}{l}\text { There will be electronic access to this } \\
\text { integrated set of ORP Requirements }\end{array}$ & $\begin{array}{l}\text { The ORP Requirements project can be } \\
\text { accessed electronically directly through } \\
\text { DOORS or through the web front-end } \\
\text { DOORSNet. }\end{array}$ \\
\hline 37 & $\begin{array}{l}\text { While the requirement data may be copied to } \\
\text { relational databases for reporting purposes, } \\
\text { the primary database will be the only place } \\
\text { where requirements can be changed. }\end{array}$ & $\begin{array}{l}\text { Changes to requirements will only be made } \\
\text { within the DOORS database. Data feeds } \\
\text { from relational databases will be } \\
\text { procedurally prohibited. }\end{array}$ \\
\hline 45 & $\begin{array}{l}\text { The system shall be capable of generating } \\
\text { reports at specific times while unattended } \\
\text { (batch reporting). }\end{array}$ & $\begin{array}{l}\text { DOORS Extension Language (DXL) can be } \\
\text { used to generate reports. Batch reporting } \\
\text { can be setup for nighttime processing. }\end{array}$ \\
\hline 50 & $\begin{array}{l}\text { The system must provide export capability } \\
\text { of all requirements data including } \\
\text { hierarchical relationships, links between } \\
\text { requirements, and user-defined attributes to } \\
\text { the relational databases (such as Access, } \\
\text { SQL Server, and Oracle). }\end{array}$ & $\begin{array}{l}\text { DOORS provides Export to SQL } \\
\text { functionality. This functionality will not be } \\
\text { exercised unless specifically requested. }\end{array}$ \\
\hline 59 & $\begin{array}{l}\text { The system must have the capability to store } \\
\text { an Approval Authority Matrix that executes } \\
\text { the threshold criteria for approving a change. } \\
\text { For each authorization level, the matrix may } \\
\text { have selected attributes based on risk; scope, } \\
\text { schedule, and cost to determine change the } \\
\text { criteria or threshold. By using varying } \\
\text { change classes, assurance is provided that } \\
\text { the processing, review, and disposition of } \\
\text { changes to requirements are consistent with } \\
\text { the significance of the change and the }\end{array}$ & $\begin{array}{l}\text { DOORS can store the Approval Authority } \\
\text { Matrix. DOORS structure allows change } \\
\text { class tracking, but does not control the } \\
\text { actual changes. }\end{array}$ \\
\hline
\end{tabular}




\begin{tabular}{|c|c|c|}
\hline No. & Requirement & Resolution \\
\hline & approvers(s). & \\
\hline 73 & $\begin{array}{l}\text { Impact analysis assessment requires the } \\
\text { judgment and technical analysis of experts. } \\
\text { It generally entails determining the cost and } \\
\text { schedule impacts of changes to work scope. } \\
\text { The PIO will be dependent on the } \\
\text { contractors to perform much of the impact } \\
\text { analysis. }\end{array}$ & $\begin{array}{l}\text { Impact analysis is performed through human } \\
\text { interface. }\end{array}$ \\
\hline 88 & $\begin{array}{l}\text { Ad hoc reporting must not require the } \\
\text { services of a computer programmer. }\end{array}$ & $\begin{array}{l}\text { DOORS provides reporting capabilities that } \\
\text { allow the user to filter and define the view to } \\
\text { be reported on. }\end{array}$ \\
\hline 89 & $\begin{array}{l}\text { The occasional user who wants to be able to } \\
\text { view subsets of requirements must not } \\
\text { require any training. }\end{array}$ & $\begin{array}{l}\text { General use of DOORS will be via } \\
\text { DOORSNet, which requires minimal } \\
\text { introduction for use. }\end{array}$ \\
\hline 90 & $\begin{array}{l}\text { Training for data editing shall take less than } \\
3 \text { days. }\end{array}$ & $\begin{array}{l}\text { "Applying DOORS" training takes two days } \\
\text { to complete. }\end{array}$ \\
\hline 92 & $\begin{array}{l}\text { The system must provide data backup and } \\
\text { restore capabilities. }\end{array}$ & $\begin{array}{l}\text { DOORS provides the ability to archive and } \\
\text { restore modules and projects which can be } \\
\text { used for backup purposes. Backup of } \\
\text { DOORS data will be performed through } \\
\text { network server back ups. }\end{array}$ \\
\hline 100 & $\begin{array}{l}\text { View only access to the requirements } \\
\text { database will be available to all those } \\
\text { involved in requirements management. }\end{array}$ & $\begin{array}{l}\text { General use of DOORS will be via } \\
\text { DOORSNet, which requires minimal } \\
\text { introduction for use. }\end{array}$ \\
\hline 101 & $\begin{array}{l}\text { This view only access must be provided at } \\
\text { no cost to the viewer from their workstation. }\end{array}$ & $\begin{array}{l}\text { General use of DOORS will be via } \\
\text { DOORSNet, which is accessed through the } \\
\text { user's internet browser. }\end{array}$ \\
\hline 102 & $\begin{array}{l}\text { No more than } 1 \text { hour of training will be } \\
\text { required. }\end{array}$ & $\begin{array}{l}\text { General use of DOORS will be via } \\
\text { DOORSNet, which requires minimal } \\
\text { introduction for use. }\end{array}$ \\
\hline 105 & No training must be necessary. & $\begin{array}{l}\text { General use of DOORS will be via } \\
\text { DOORSNet, which requires minimal } \\
\text { introduction for use. }\end{array}$ \\
\hline 109 & $\begin{array}{l}\text { No more than } 2 \text { days of training will be } \\
\text { necessary }\end{array}$ & $\begin{array}{l}\text { "Applying DOORS" training is requires two } \\
\text { days to complete. }\end{array}$ \\
\hline 111 & $\begin{array}{l}\text { No more than } 2 \text { days of training will be } \\
\text { necessary. }\end{array}$ & $\begin{array}{l}\text { "Applying DOORS" training is requires two } \\
\text { days to complete. Two hour overview } \\
\text { sessions will be provided for the } \\
\text { DOORSNet users. }\end{array}$ \\
\hline 112 & $\begin{array}{l}\text { The system must be available } 10 \text { hours per } \\
\text { day, } 7 \text { days a week from } 7 \text { a.m. }-5 \text { p.m. }\end{array}$ & $\begin{array}{l}\text { DOORS servers are available during core } \\
\text { business hours } 7 \text { a.m. }-5 \text { p.m. standard work } \\
\text { days. }\end{array}$ \\
\hline 113 & $\begin{array}{l}\text { The software must execute in a client server } \\
\text { or web environment. }\end{array}$ & $\begin{array}{l}\text { DOORS executes in a client server } \\
\text { environment. DOORSNet executes in a web } \\
\text { environment. }\end{array}$ \\
\hline$\Pi 14$ & $\begin{array}{l}\text { The client portion of the tool must be } \\
\text { compatible with Windows } 95 / 98 / \mathrm{NT} \text {. }\end{array}$ & $\begin{array}{l}\text { DOORS client is compatible with Windows } \\
95 / 98 / \mathrm{NT} \text {. }\end{array}$ \\
\hline
\end{tabular}




\begin{tabular}{|l|l|l|}
\hline No. & \multicolumn{1}{|c|}{ Requirement } & \multicolumn{1}{|c|}{ Resolution } \\
\hline 115 & $\begin{array}{l}\text { The server portion of the tool must run in an } \\
\text { NT environment }\end{array}$ & $\begin{array}{l}\text { DOORS server portion runs in an NT } \\
\text { environment. }\end{array}$ \\
\hline 117 & $\begin{array}{l}\text { All data must be portable to the following } \\
\text { relational databases: Access, SQL Server, } \\
\text { and Oracle. }\end{array}$ & $\begin{array}{l}\text { DOORS provides Export to SQL } \\
\text { functionality. This functionality will not be } \\
\text { exercised unless specifically requested. }\end{array}$ \\
\hline 118 & $\begin{array}{l}\text { The Network license policy must be } \\
\text { acceptable to the Chief Information Officer. }\end{array}$ & $\begin{array}{l}\text { RPP CIO approves the Network licensing } \\
\text { policy for DOORS. }\end{array}$ \\
\hline 119 & $\begin{array}{l}\text { Software updates must be released and } \\
\text { implemented with less than 4 hours of user } \\
\text { downtime. }\end{array}$ & $\begin{array}{l}\text { Software updates will be implemented in } \\
\text { less than 4 hours of user downtime. }\end{array}$ \\
\hline 120 & $\begin{array}{l}\text { Vendor must allow reproduction of training } \\
\text { materials for general user training. }\end{array}$ & $\begin{array}{l}\text { lf needed, an arrangement will be made with } \\
\text { the vendor, QSS. }\end{array}$ \\
\hline 124 & The software must be Y2K Compliant. & DOORS \& DOORSNet are Y2K compliant \\
\hline 125 & The software must be customizable. & $\begin{array}{l}\text { DOORS is customizable with the DOORS } \\
\text { Extension Language (DXL). }\end{array}$ \\
\hline
\end{tabular}

\subsection{Deliverables}

This test plan including associated test cases and results will be provided as the deliverable for acceptance testing. Customer acceptance signatures will be included in this test plan.

\subsection{Acceptance Criteria}

Test results should meet resolution of requirements completely. In any other instance, test cases and results will be reviewed with the customer. Acceptance of test plan, cases, results, and exceptions will be indicated through signatures within this plan. 


\section{TESTING LOGISTICS}

\subsection{Testing Tasks}

In preparation for the performance of test cases included in this plan, testing will be scheduled, a testing location will be confirmed, testing materials will be prepared, and communication of details will be made to all testing parties.

Data conversion will not be required to perform the test cases. Input will be performed manually. In the case of import testing, standard electronic files will be made available to all testers.

\subsection{Environmental Needs}

The following environmental needs have been identified for this test plan:

\begin{tabular}{|c|c|}
\hline Personnel & $\begin{array}{l}\text { LMSI - All of the technical team will participate in the testing } \\
\text { PIO - Requirements Management team members will be invited to } \\
\text { participate in the testing }\end{array}$ \\
\hline Facilities & A single location will be arranged for testing. \\
\hline Equipment & Several workstations will be available for testing. \\
\hline Time Allocation & Two complete days will be reserved for testing. \\
\hline
\end{tabular}

\subsection{Staffing and Training}

All of the LMSI DOORS technical team will participate in the testing. The Requirements Management team members will be invited to participate in the testing. All testers will be expected to have attended DOORS training or at least have a familiarity of the application.

\subsection{Schedule}

The following are the DOORS implementation testing milestones.

Test Plan Draft Review

Test Plan Approval

Perform Test Cases

Acceptance Sign-off
March 31, 2000

April 7, 2000

April 10, 2000 - April 13, 2000

April 13, 2000

\subsection{Risks and Contingencies}

No high-level risks are identified for the testing. 


\section{ROLES AND RESPONSIBILITIES}

\subsection{Requirements Management Team - System Users}

Users will be invited to perform the test cases that test those requirements that they directly utilize during the course of their normal user activities. They will not normally conduct acceptance testing of those requirements that occur behind the scenes, such as automated feed processes.

\subsection{Lockheed Martin Services, Inc. - DOORS Technical Team}

LMSI staff will perform all test cases. The LMSI staff will also be responsible for testing items that occur behind the scenes and which are not visible to the user. Examples would be licensing allocation, server functions, or automated batch processes that are not user options in the application.

\subsection{Project Management \& Lead Responsibilities}

Projects Managers and Leads from both LMSI SD\&I and the PIO Requirements Management Team are expected to review test results and provide guidance, direction, and suggestions for improving the testing process. Their acceptance signatures will be included within this test plan. 
RPP-6251 REV. 0

\section{TESTING SUMMARY RESULTS}

Testing summary results for the PIO DOORS implementation project were positive. Although no users elected to perform testing, LMSI DOORS technical staff performed each test case at least twice.

Testing participants included:

- Adria Johnson, LMSI

- Jennifer Kuklinski, LMSI

- Steve McConnell, LMSI

- Rick Strickland, LMSI

- Joyce Caldwell, LMSI

The following anomalies discovered during testing do not seriously affect system use. The issues be investigated and resolved.

\begin{tabular}{|c|l|l|}
\hline Test Case \# & \multicolumn{1}{|c|}{ Issue } & \multicolumn{1}{c|}{ Workaround } \\
\hline DOORS-6.04 & $\begin{array}{l}\text { DOORS Change Proposal (CP) Notify } \\
\text { Message }\end{array}$ & $\begin{array}{l}\text { CP Review Manager can reset } \\
\text { CP preferences }\end{array}$ \\
\hline DOORS-6.06 & DOORSNet Connection Error Message & $\begin{array}{l}\text { User can select "back" and } \\
\text { reload web page }\end{array}$ \\
\hline
\end{tabular}

These anomalies and other testing issues that do not affect the system are described in detail in Section 5.1, Test Case Discrepancy and Resolution Matrix. 


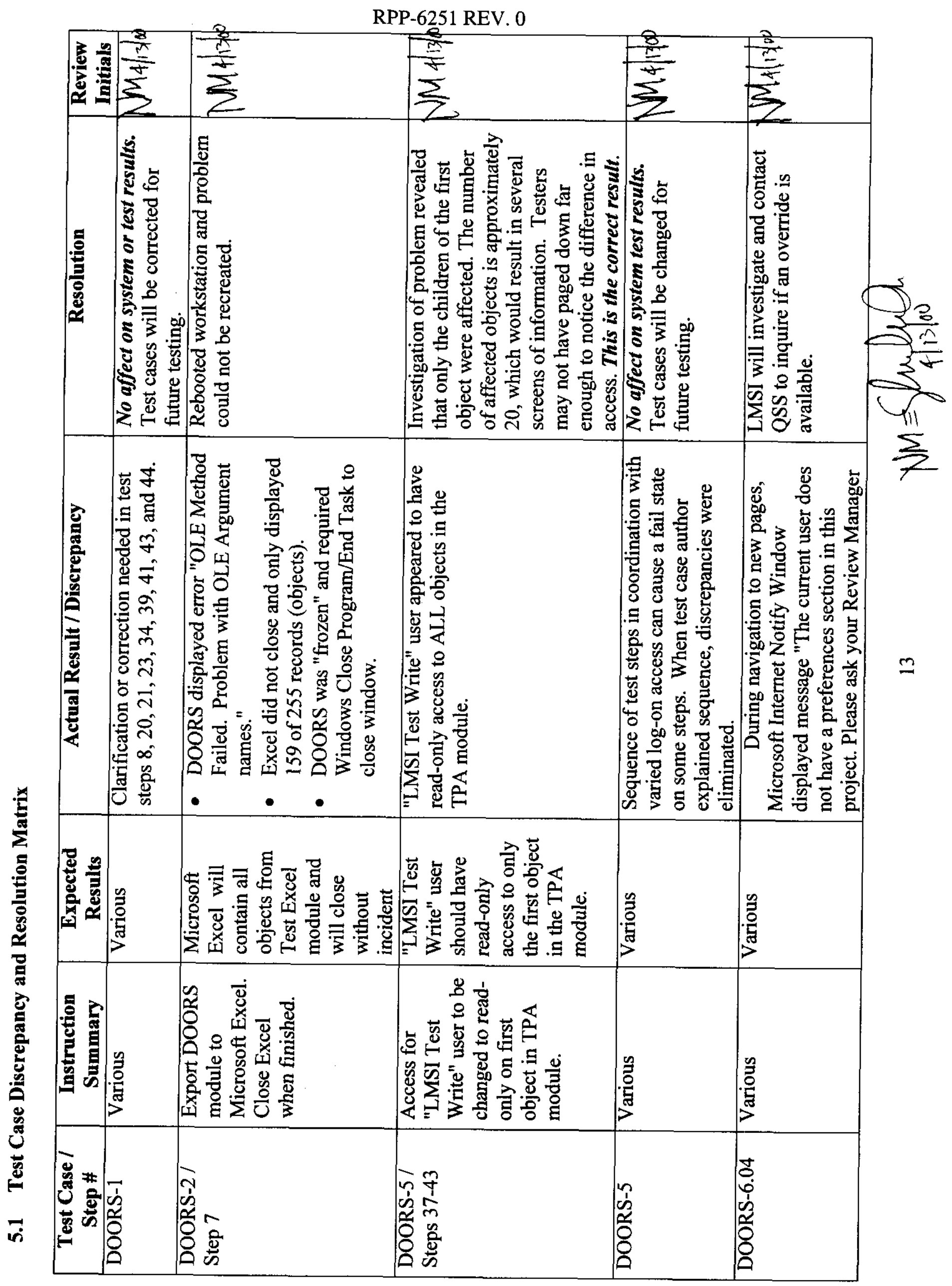


RPP-6251 REV. 0

\begin{tabular}{|c|c|c|}
\hline 苞 & & $\sum \frac{8}{2}$ \\
\hline & & 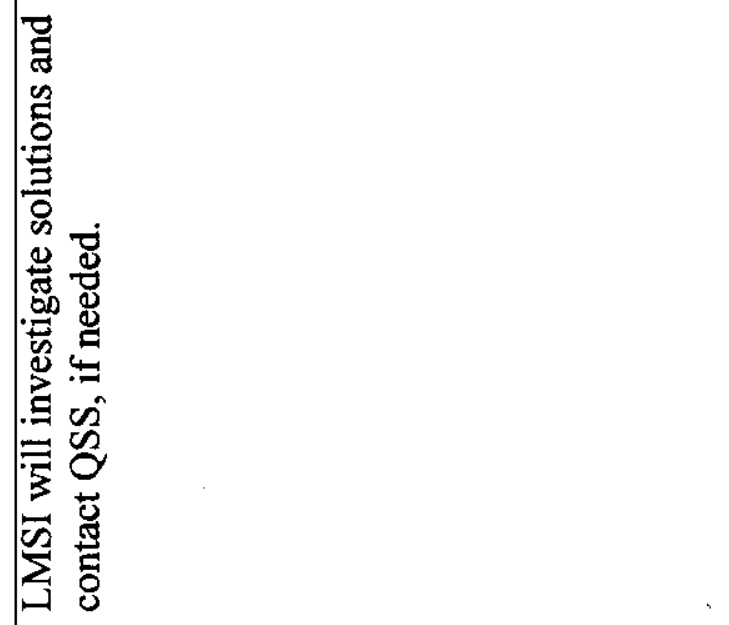 \\
\hline 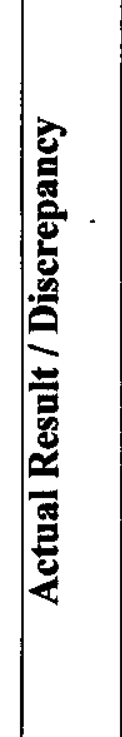 & 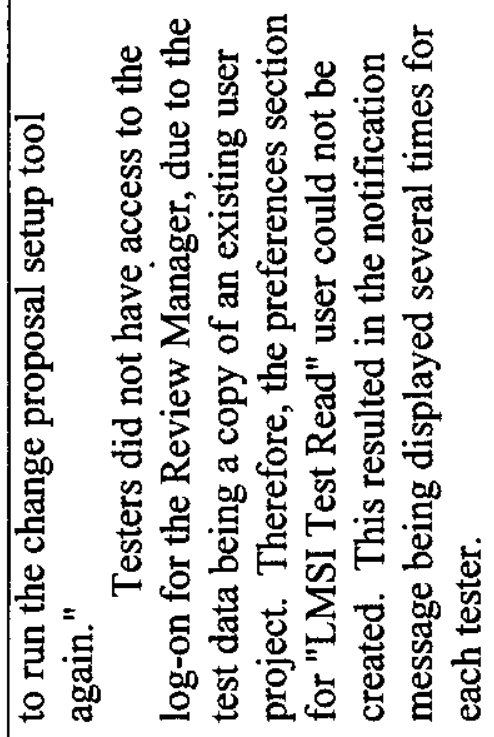 & 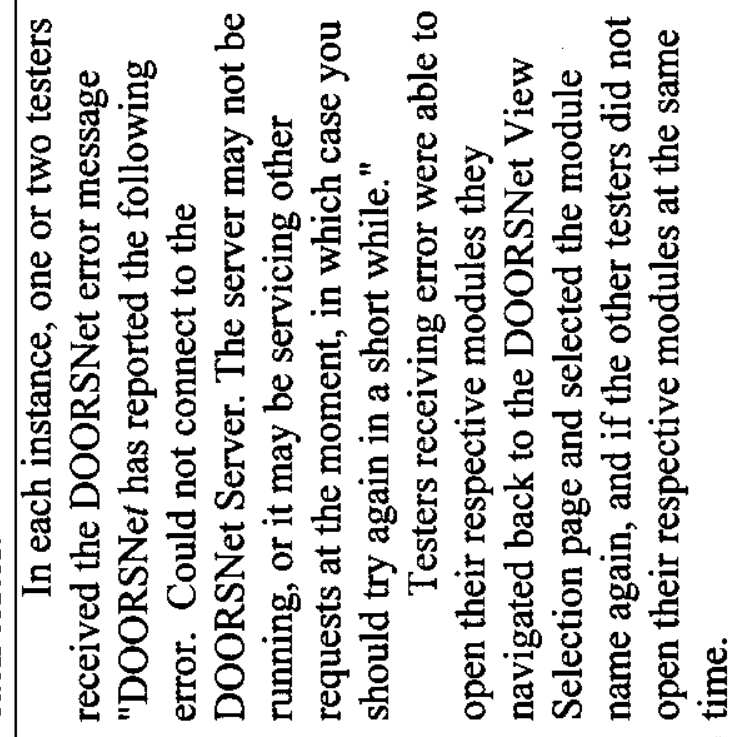 \\
\hline 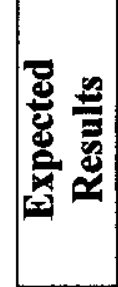 & & 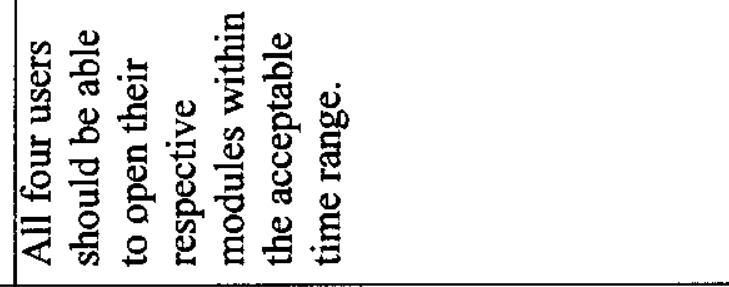 \\
\hline 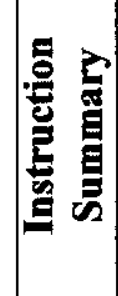 & & 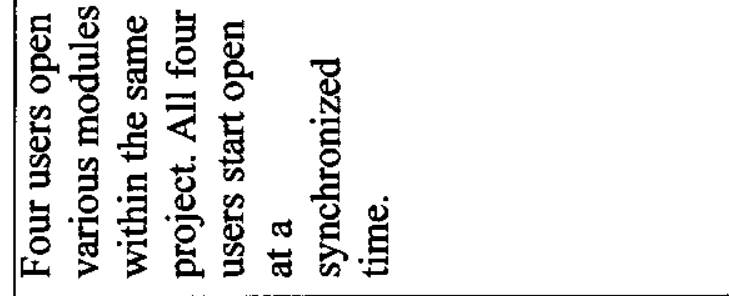 \\
\hline 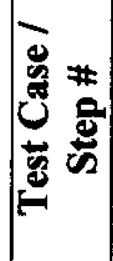 & & 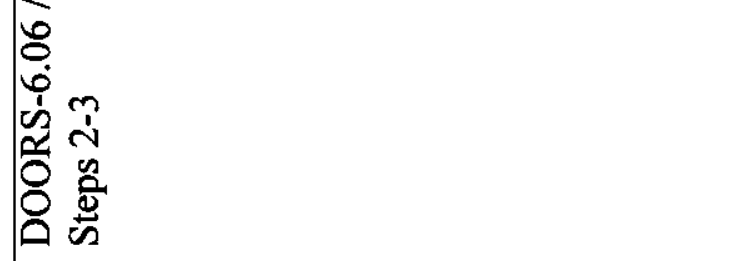 \\
\hline
\end{tabular}

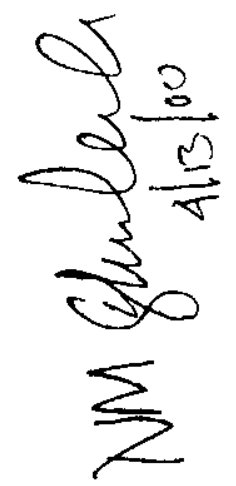




\section{CUSTOMER ACCEPTANCE}

The Dynamic Object Oriented Requirements System (DOORS) has been satisfactorily tested by LMSI personnel and/or PIO test representatives. This system is accepted by the PIO as having achieved the requirements for which Lockheed Martin Services, Inc., Richland, implemented it.

\section{Customer Acceptance}

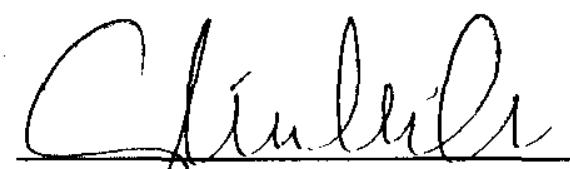

Nancy J / 1 illiken, CHG, PIO DOORS Implementation Project Manager
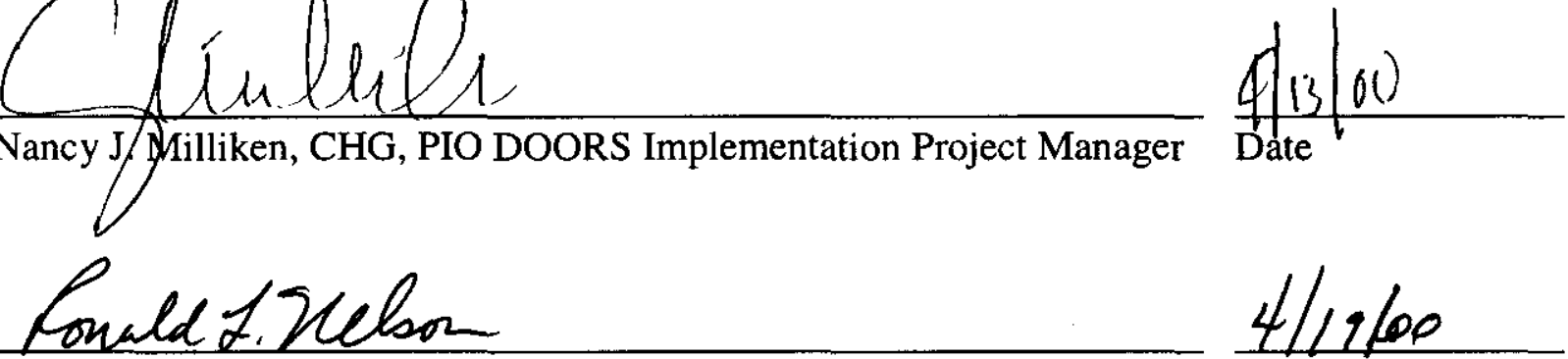

Ronald L. Nelson, CHG, PIO Chief Information Officer

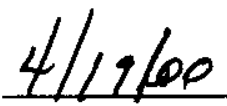

Date

\section{Performing Organization}

Tene 7u. Ettro

Terri M. Lutter, LMSI/SD\&I, Program Manager $\frac{4 \cdot 17 \cdot 00}{\text { Date }}$

Gduin R. Johuson

Adria L. Johnson, LMSI/SD\&I, Project Lead
$4-17-00$

Date 
RPP-6251 REV. 0

\begin{tabular}{|c|c|c|c|c|c|c|c|}
\hline 产 & 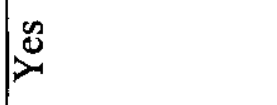 & $x_{2}^{\infty}$ & $\ddot{2}$ & $\ddot{d}$ & $\ddot{\infty}$ & $\stackrel{\infty}{\infty}$ & $\ddot{\Delta}$ \\
\hline & 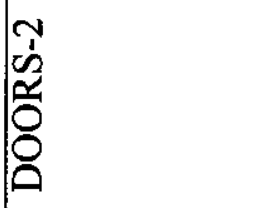 & 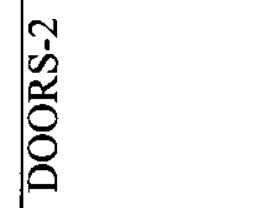 & 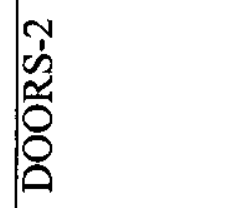 & 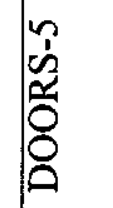 & $\mathbb{z}$ & 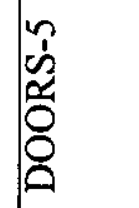 & 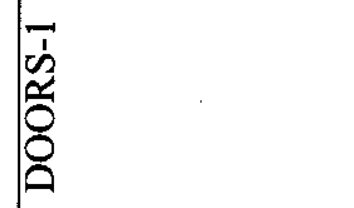 \\
\hline 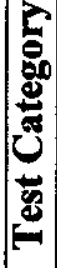 & 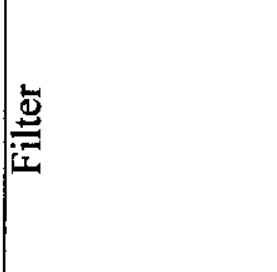 & 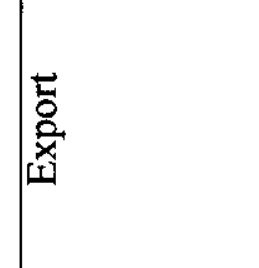 & 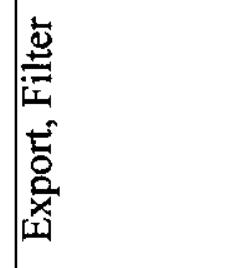 & 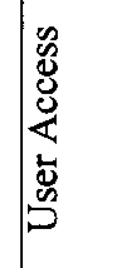 & 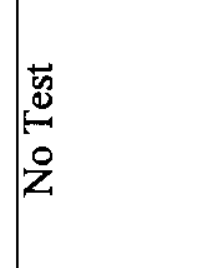 & 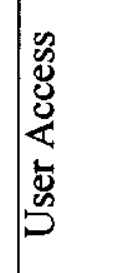 & 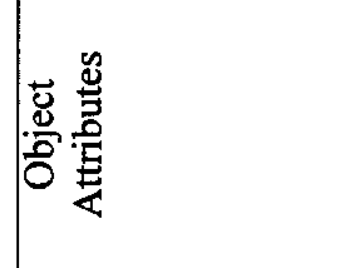 \\
\hline & 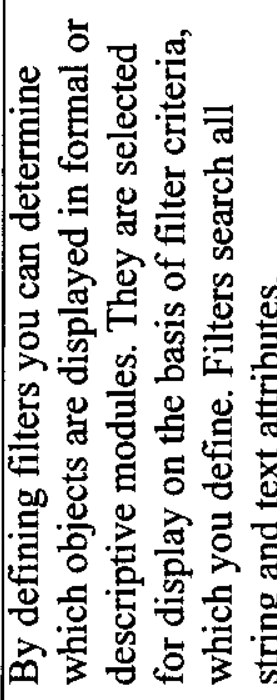 & 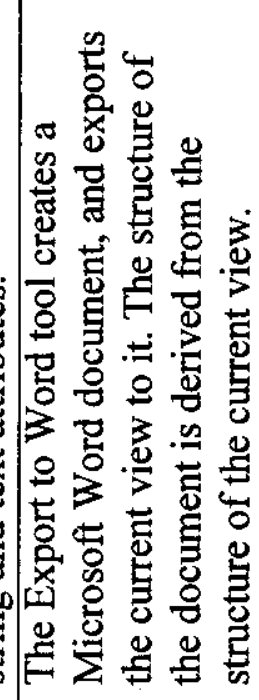 & 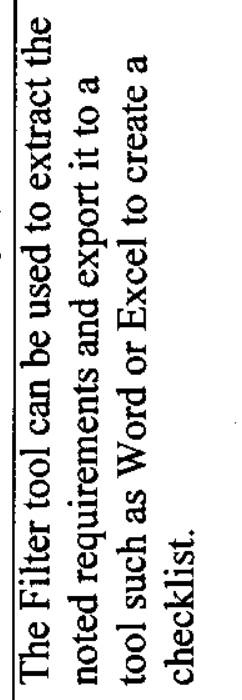 & 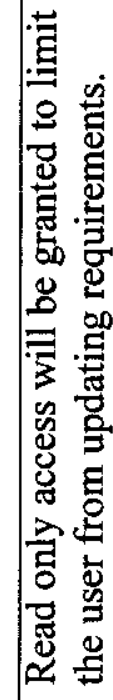 & 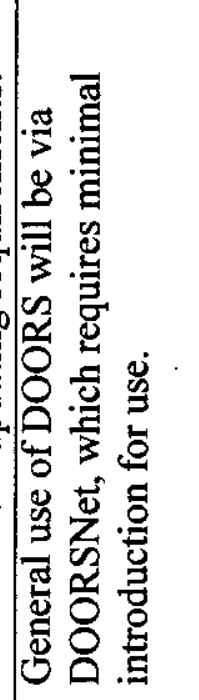 & 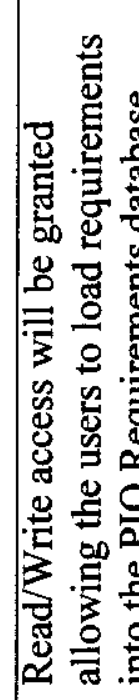 & 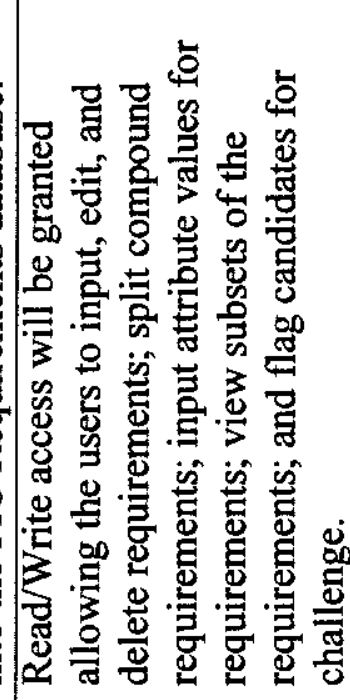 \\
\hline 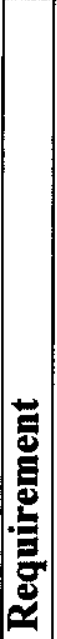 & 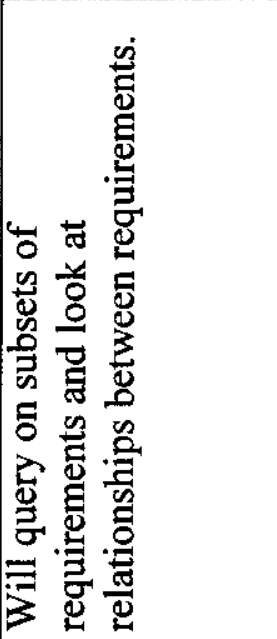 & 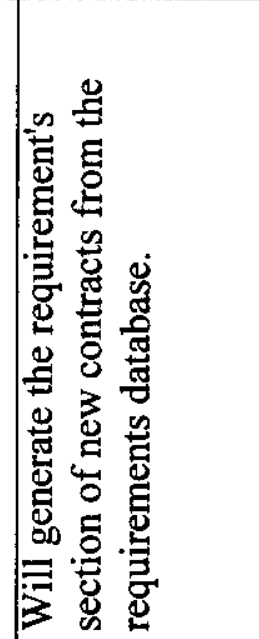 & 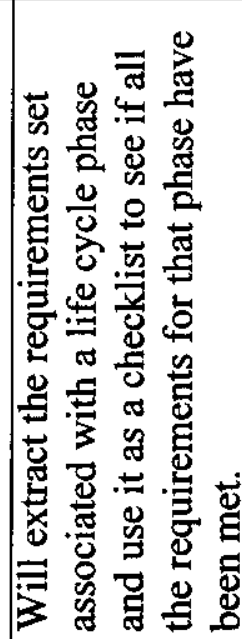 & 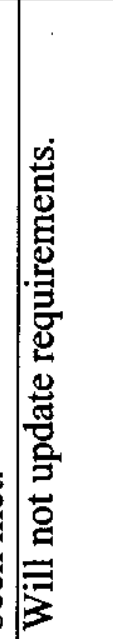 & 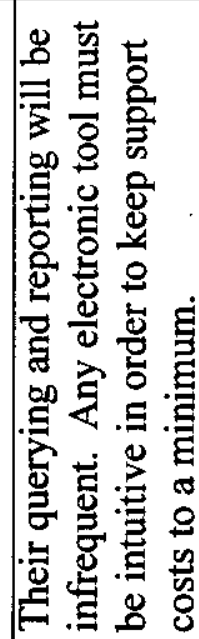 & 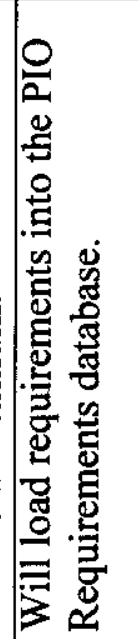 & 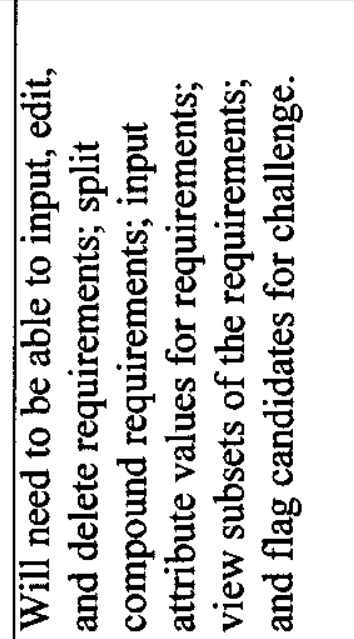 \\
\hline & 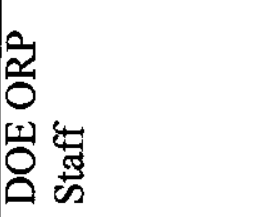 & 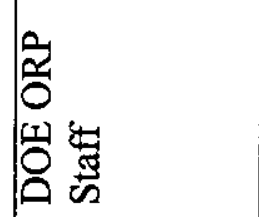 & 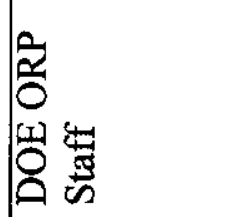 & 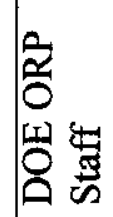 & 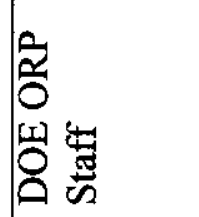 & 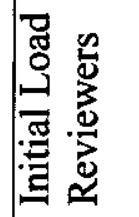 & 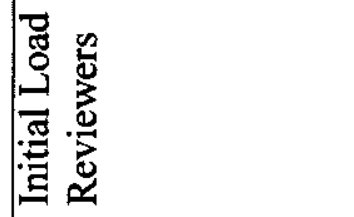 \\
\hline$\dot{z}$ & & $N$ & $m$ & $\nabla$ & in & 0 & $r$ \\
\hline
\end{tabular}


RPP-6251 REV. 0

\begin{tabular}{|c|c|c|c|c|c|c|c|c|}
\hline 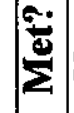 & 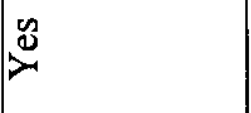 & $\stackrel{\Perp}{>}$ & 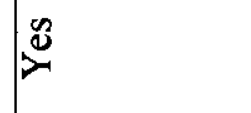 & $\stackrel{8}{\not}$ & $\stackrel{\infty}{2}$ & $\stackrel{\infty}{\infty}$ & $\not{\infty}$ & $\ddot{2}$ \\
\hline & & 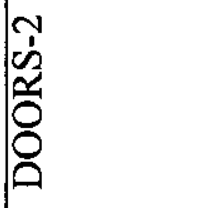 & $\mathbb{z}$ & $\varangle$ & 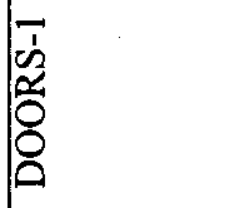 & 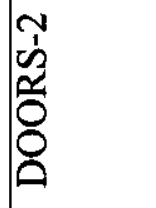 & 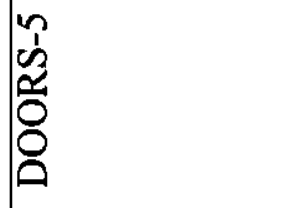 & 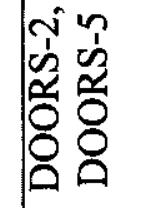 \\
\hline 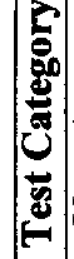 & 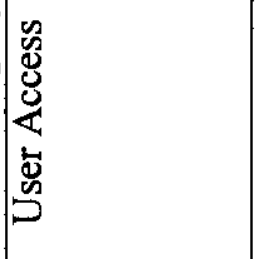 & 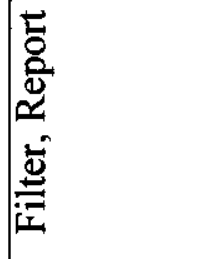 & 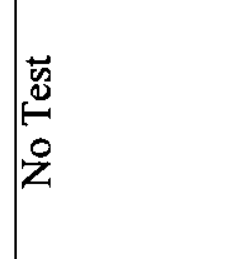 & 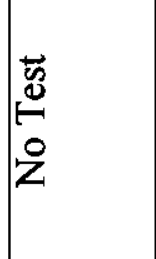 & 总 & 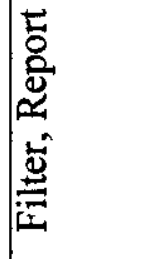 & 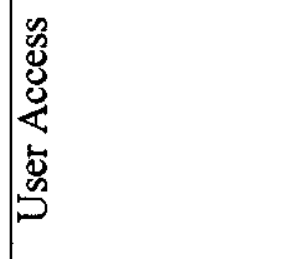 & 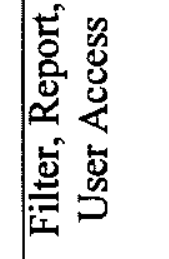 \\
\hline & 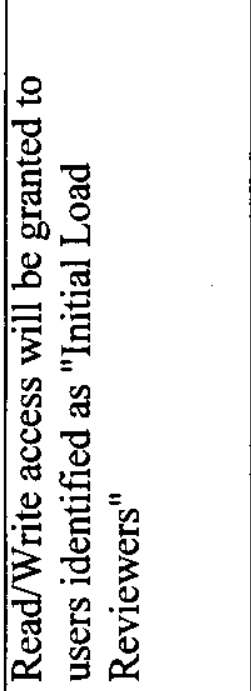 & 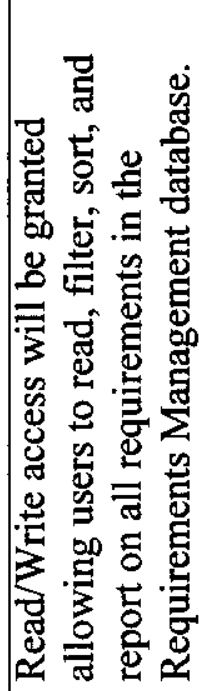 & 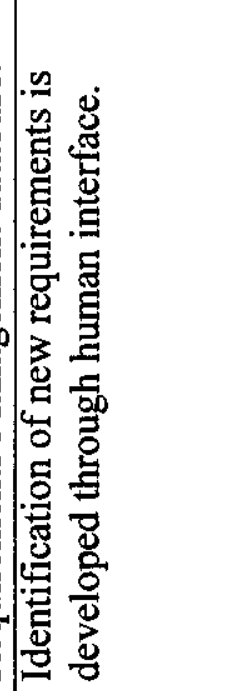 & 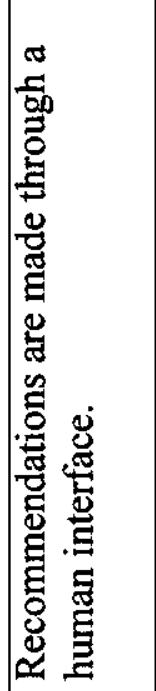 & 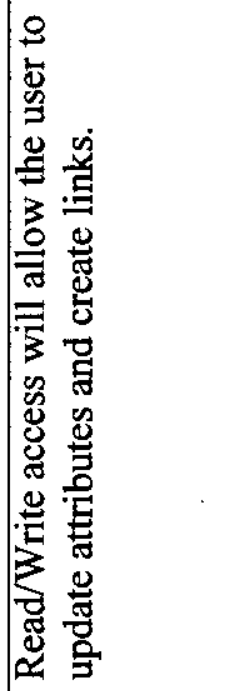 & 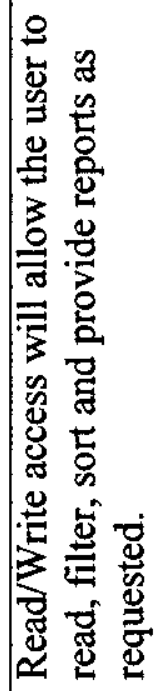 & 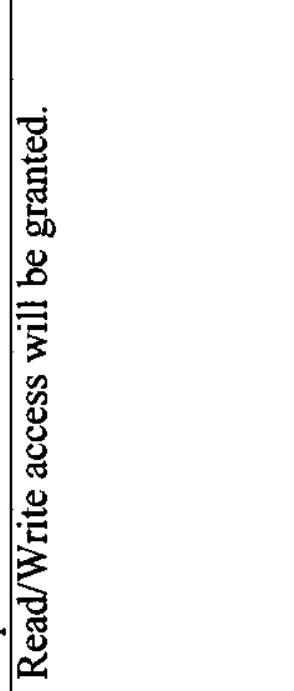 & 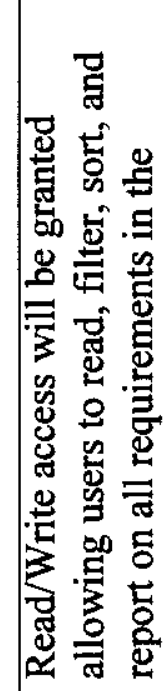 \\
\hline $\mid$ & 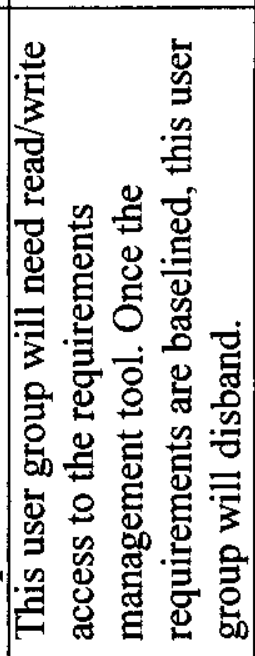 & 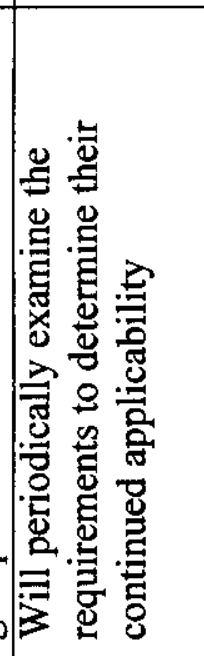 & 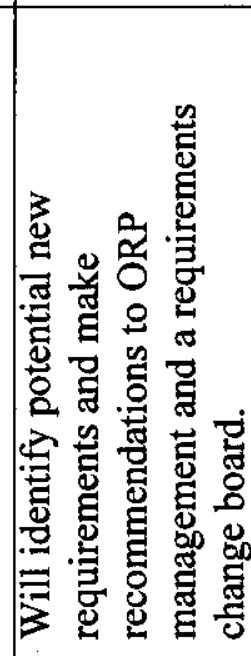 & 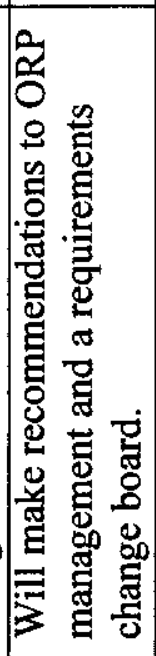 & 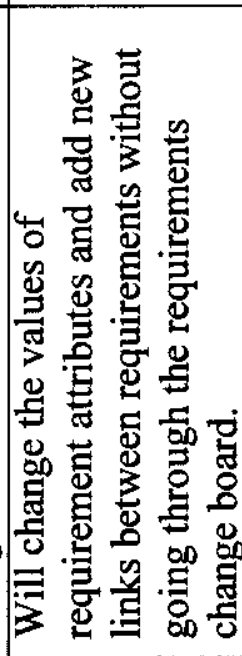 & 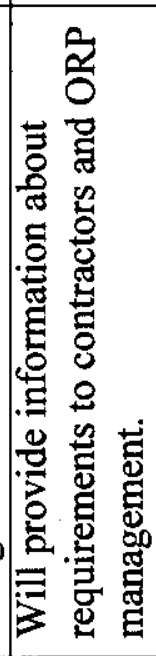 & 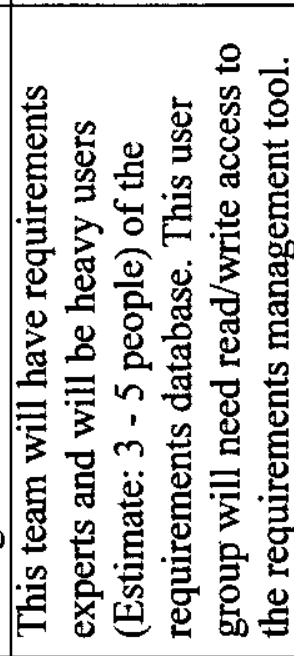 & 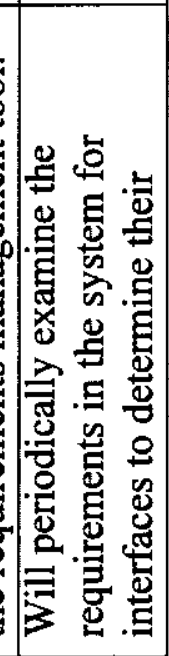 \\
\hline 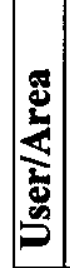 & 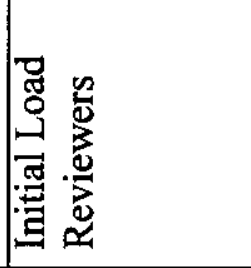 & 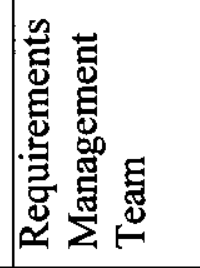 & 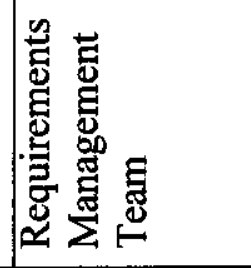 & 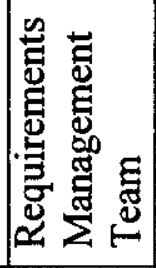 & 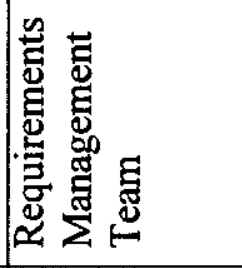 & 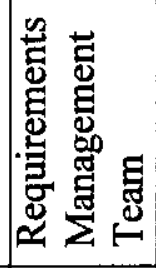 & 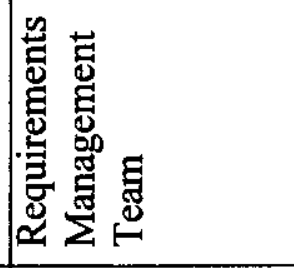 & 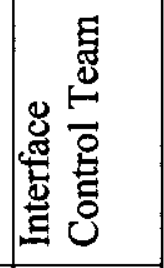 \\
\hline $\mid \mathbf{z}$ & $\infty$ & a & $\Theta$ & $\equiv$ & $\approx$ & $\approx$ & \pm & $\approx$ \\
\hline
\end{tabular}


RPP-6251 REV. 0

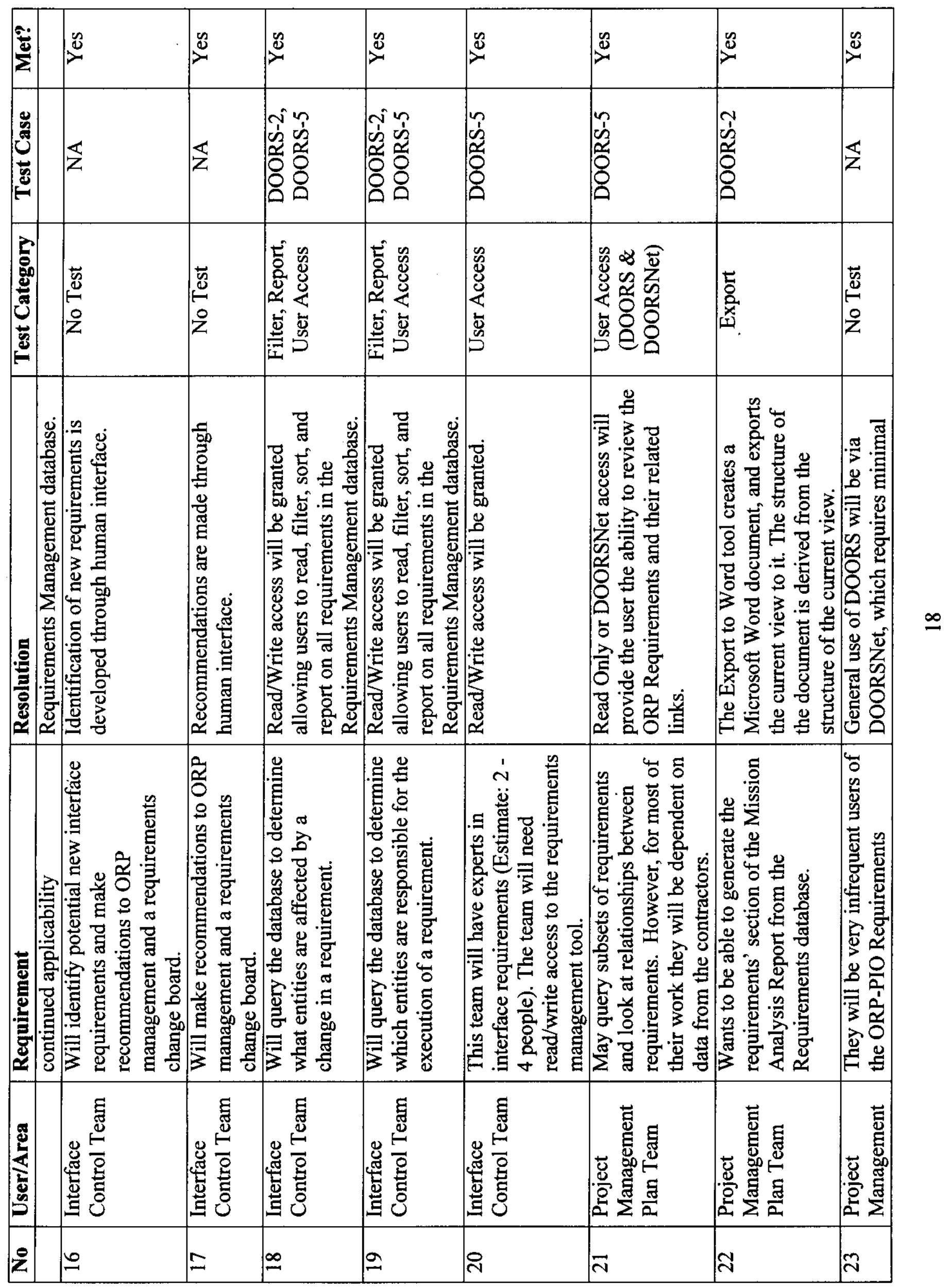


RPP-6251 REV. 0

\begin{tabular}{|c|c|c|c|c|c|c|c|}
\hline $\mid$ & $\ddot{\infty}$ & 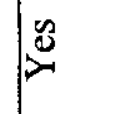 & $\nu^{\infty}$ & 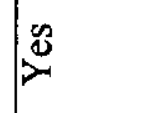 & $\nu^{\infty}$ & $\underset{\infty}{2}$ & $\stackrel{\mathscr{d}}{2}$ \\
\hline 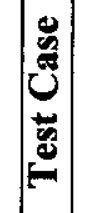 & $\mathbb{z}$ & 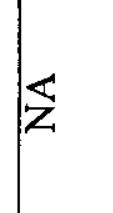 & $\begin{array}{l}n \\
\dot{n} \\
\tilde{\alpha} \\
0 \\
0 \\
0\end{array}$ & $\mathbb{z}$ & 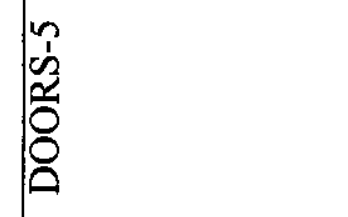 & 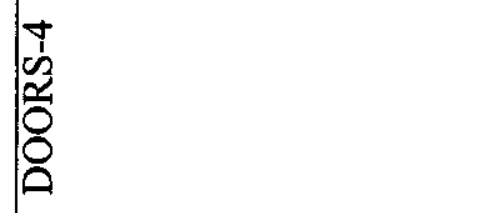 & 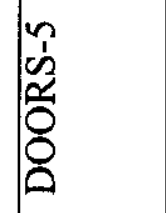 \\
\hline 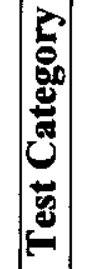 & 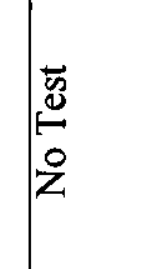 & 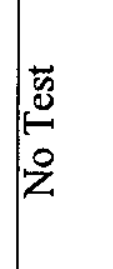 & 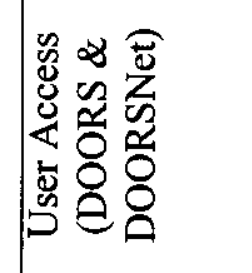 & 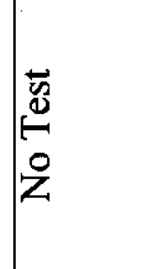 & 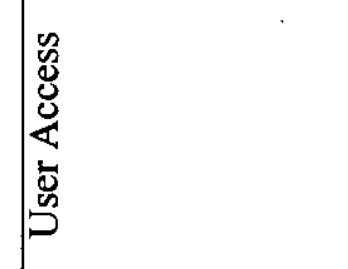 & 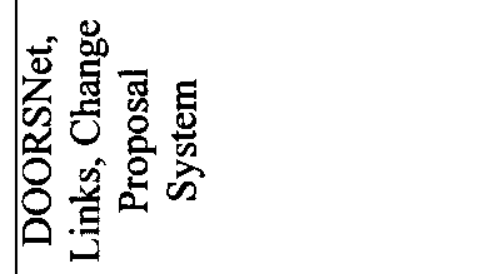 & 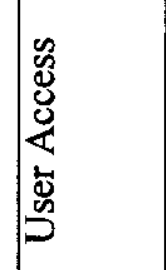 \\
\hline 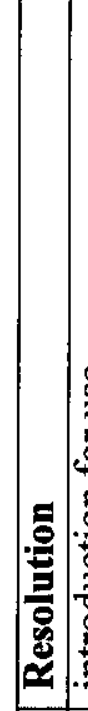 & 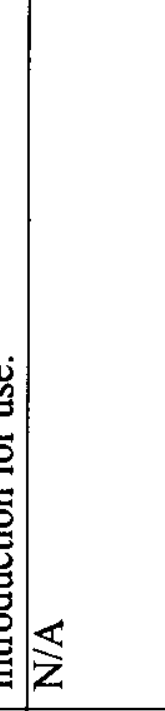 & 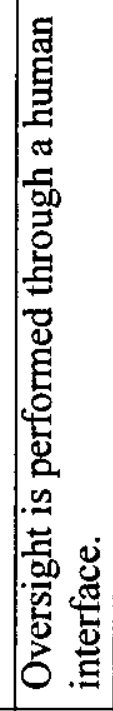 & 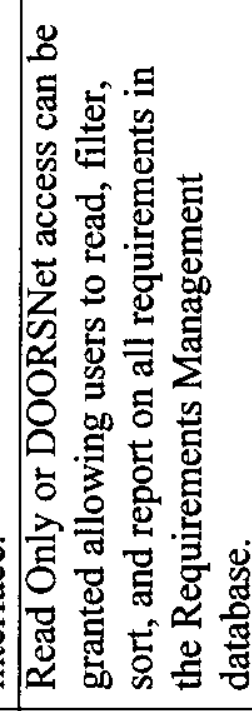 & 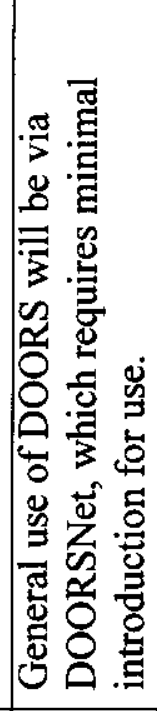 & 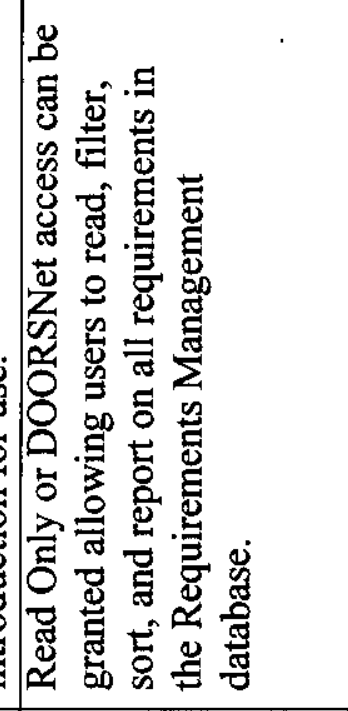 & 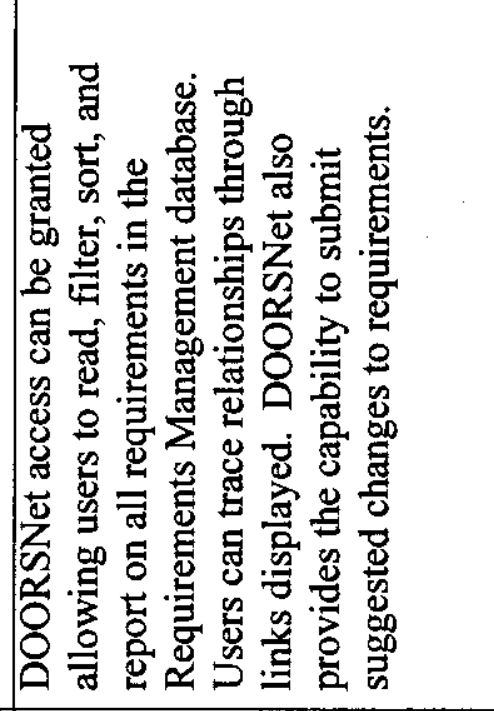 & 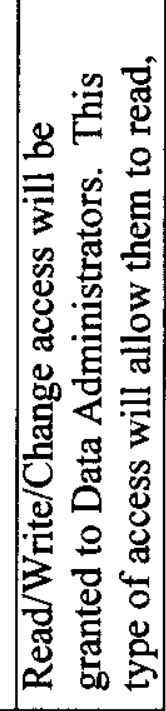 \\
\hline 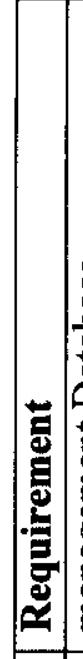 & 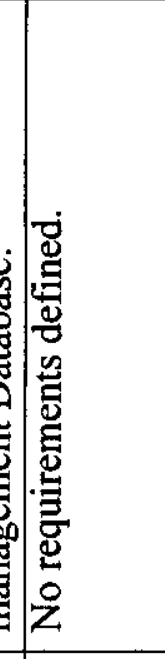 & 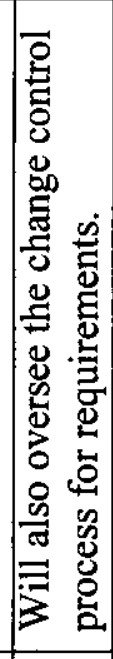 & 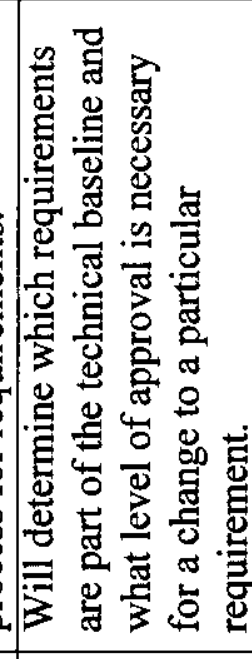 & 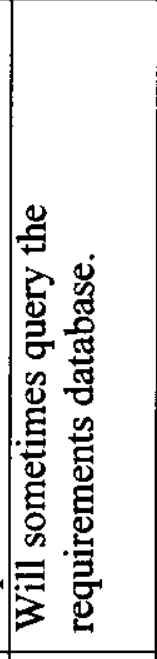 & 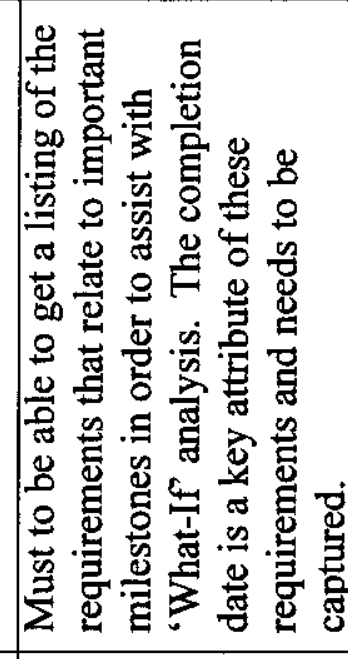 & 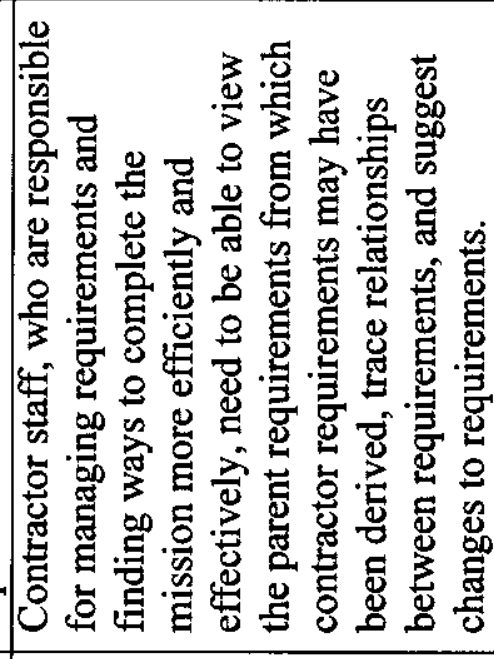 & 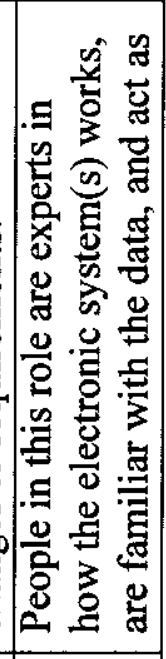 \\
\hline 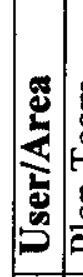 & 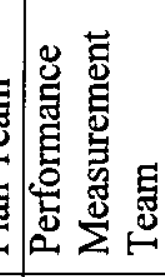 & 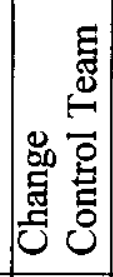 & 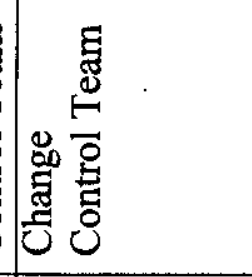 & 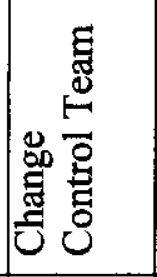 & 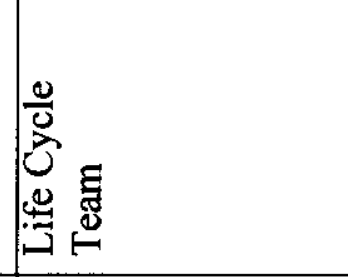 & 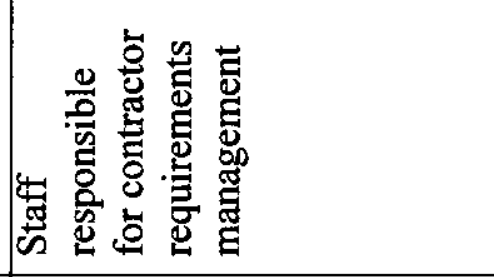 & 总 \\
\hline i & 志 & $\approx$ & $\stackrel{\infty}{*}$ & $\hat{\mathrm{N}}$ & $\underset{N}{\infty}$ & 尺े & 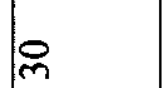 \\
\hline
\end{tabular}


RPP-6251 REV. 0

\begin{tabular}{|c|c|c|c|c|c|c|}
\hline 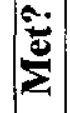 & & $\stackrel{\infty}{\infty}$ & 2 & $\ddot{\nu}$ & $\stackrel{\infty}{\infty}$ & $\nu^{\infty}$ \\
\hline $\mid$ & & $\begin{array}{l}n \\
\hat{n} \\
\tilde{z} \\
\delta \\
\delta\end{array}$ & $\mathbb{z}$ & 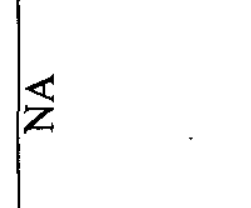 & 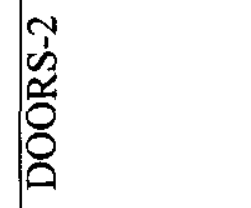 & 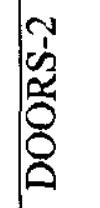 \\
\hline 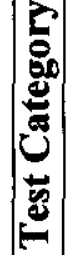 & & 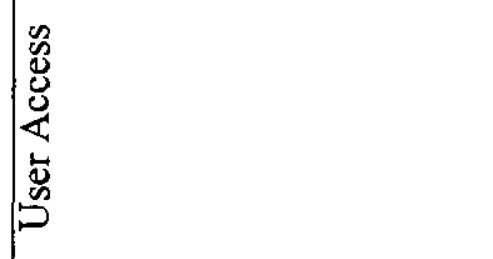 & 葡 & 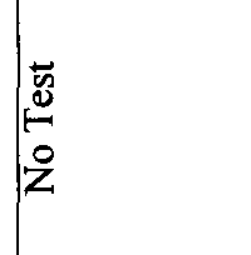 & 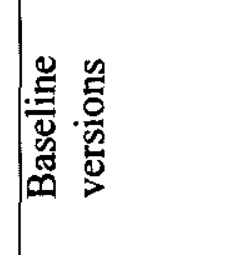 & 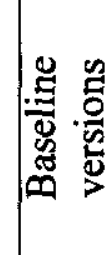 \\
\hline 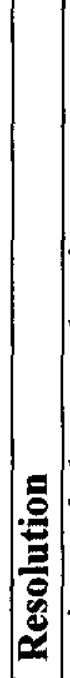 & 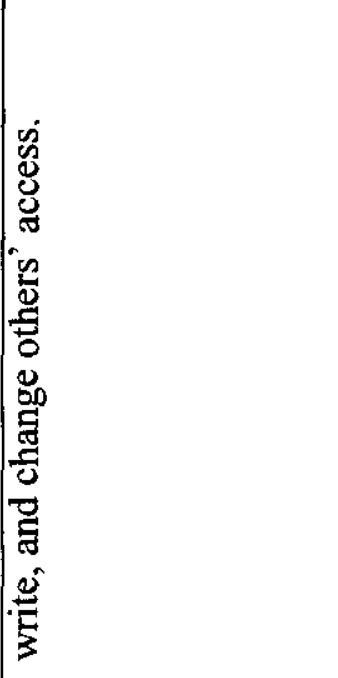 & 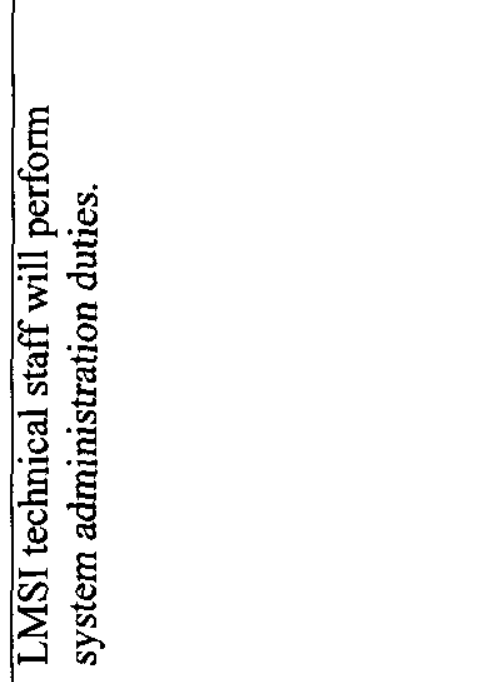 & 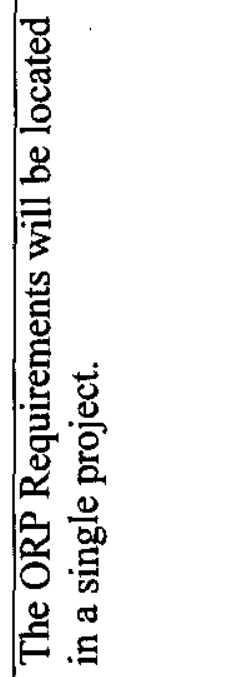 & 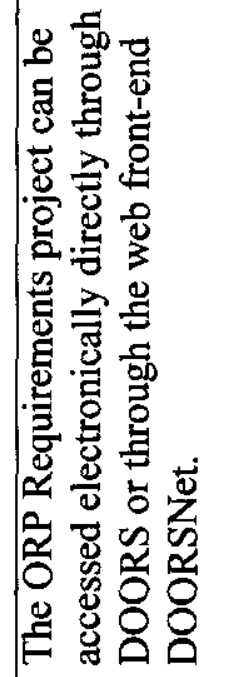 & 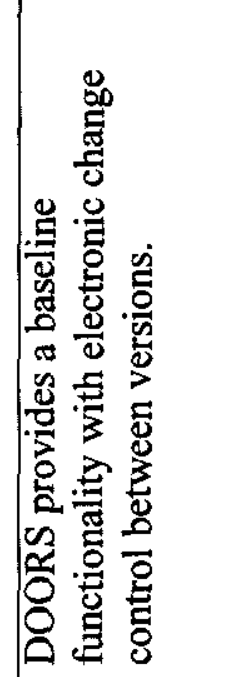 & 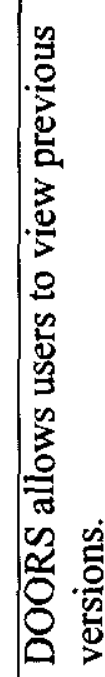 \\
\hline 萢 & 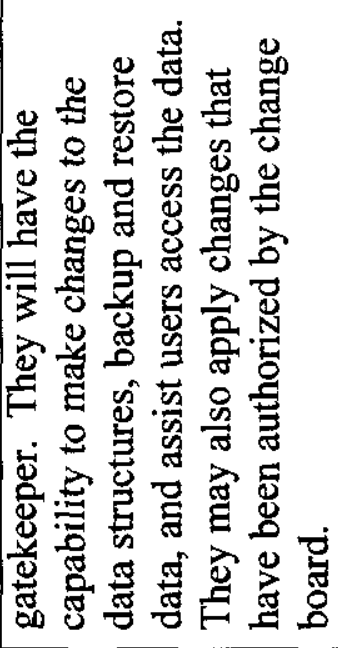 & 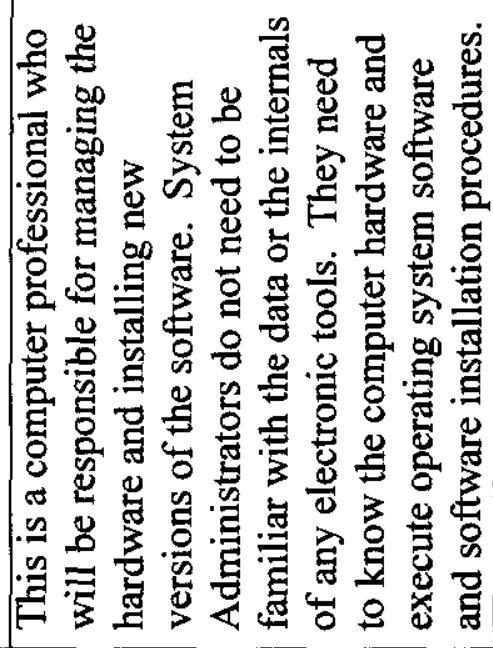 & 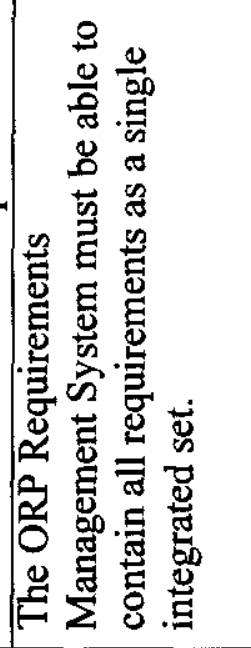 & 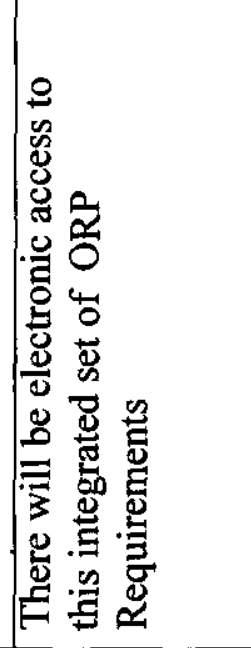 & 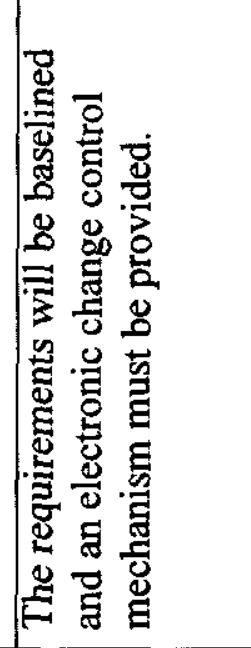 & 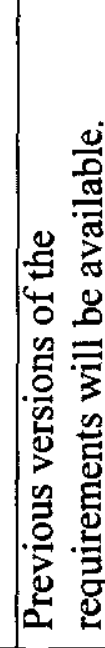 \\
\hline 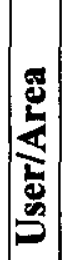 & & | & 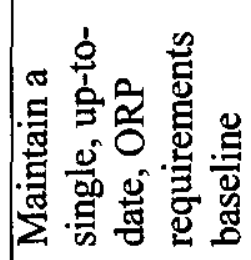 & 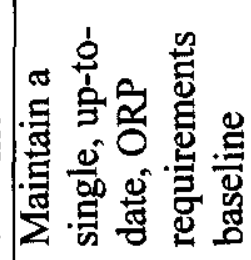 & 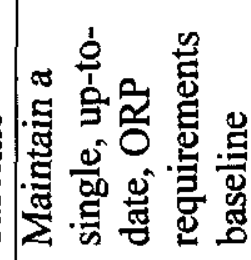 & 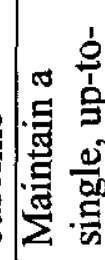 \\
\hline $\mathbf{z}$ & & $\vec{m}$ & $\tilde{N}$ & $m$ & মি & $m$ \\
\hline
\end{tabular}


RPP-6251 REV. 0

\begin{tabular}{|c|c|c|c|c|c|c|c|}
\hline | & & $\infty^{\infty}$ & $\sum^{\infty}$ & \rangle$^{\infty}$ & $\underset{\mathscr{e}}{\not}$ & $y^{\infty}$ & $\overbrace{}^{\infty}$ \\
\hline 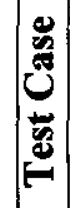 & & 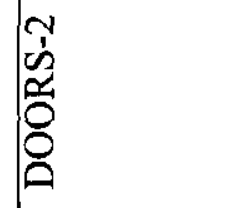 & $\mathbb{Z}$ & 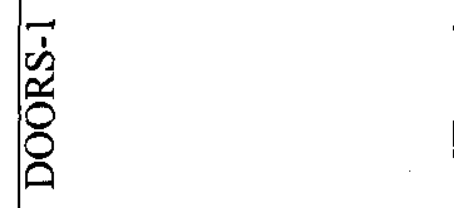 & 管 & 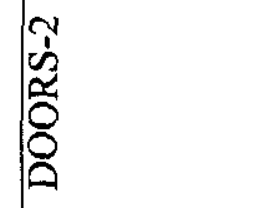 & $\begin{array}{l}\tilde{r} \\
\tilde{2} \\
\tilde{z} \\
\delta \\
0\end{array}$ \\
\hline 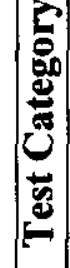 & & 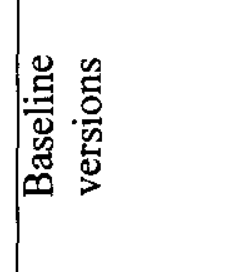 & 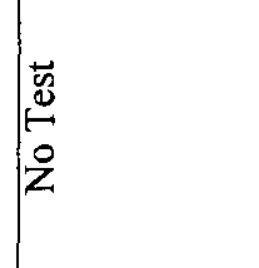 & 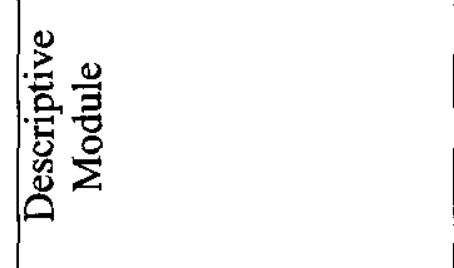 & 总 & 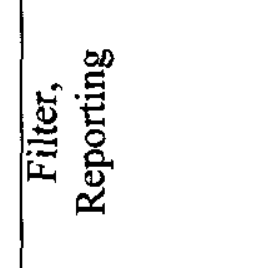 & 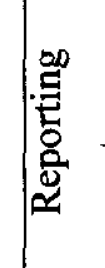 \\
\hline 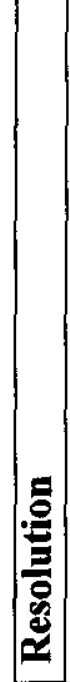 & & 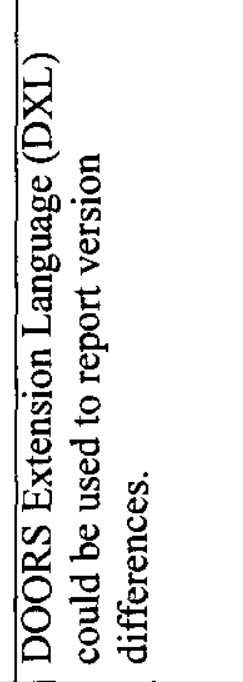 & 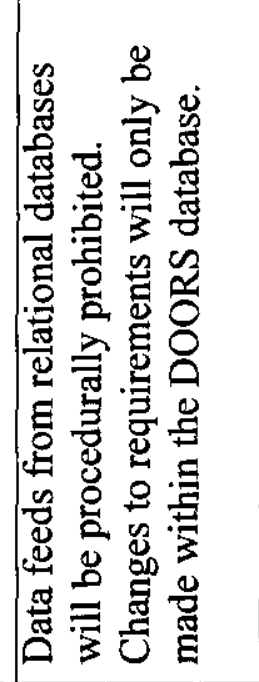 & 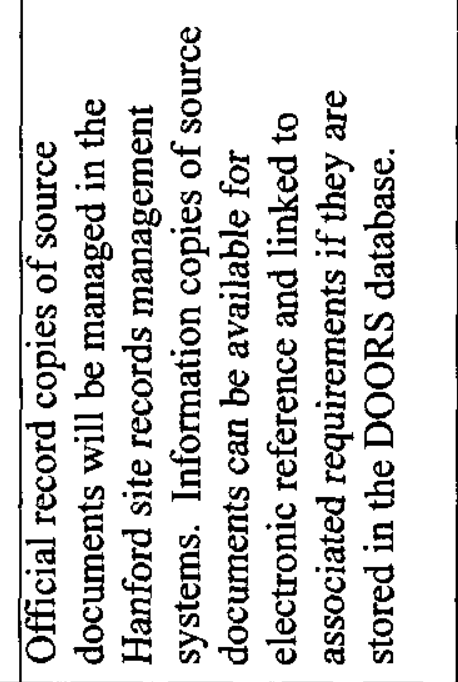 & 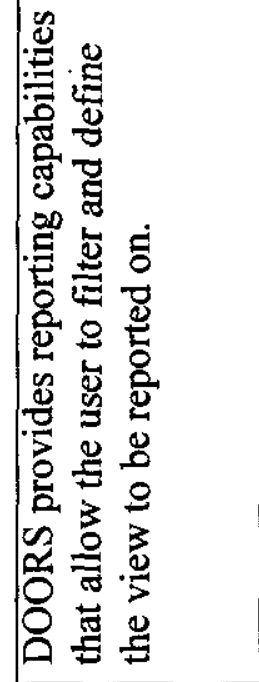 & 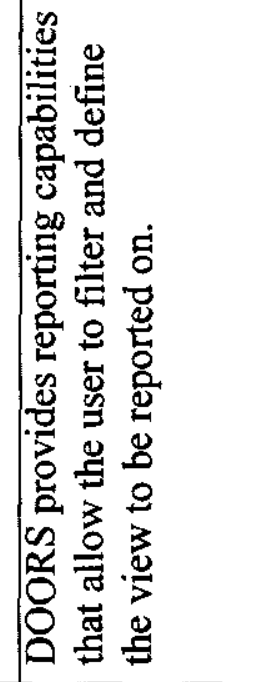 & 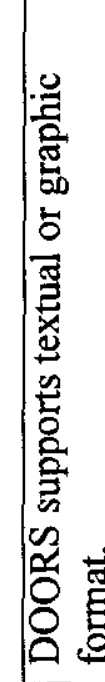 \\
\hline 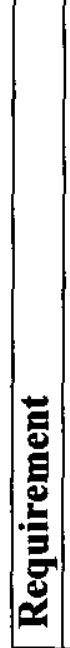 & & 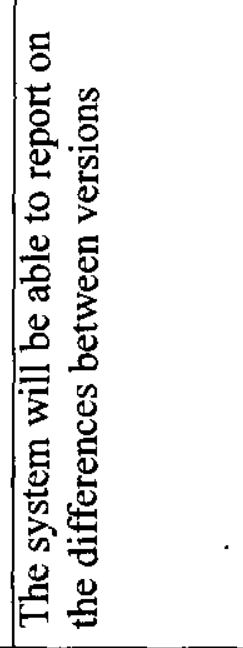 & 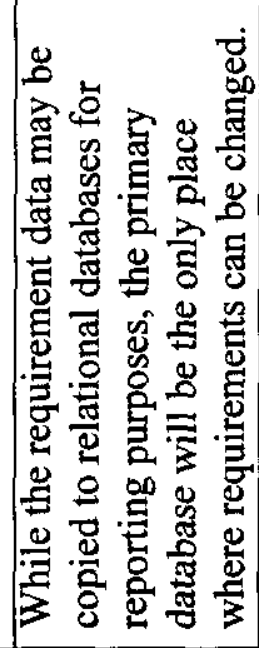 & 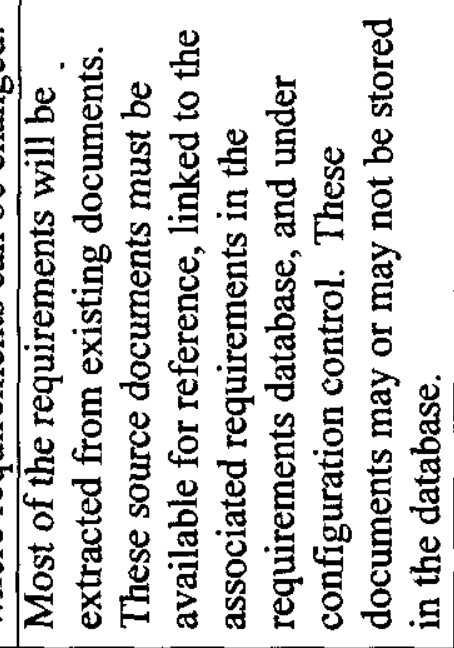 & 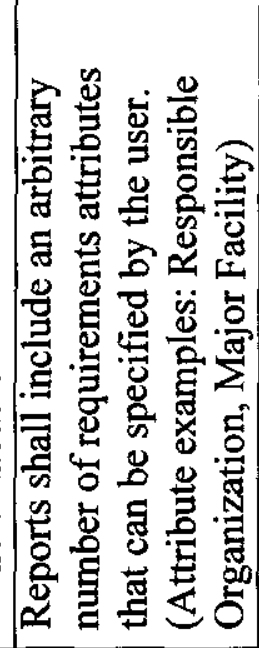 & 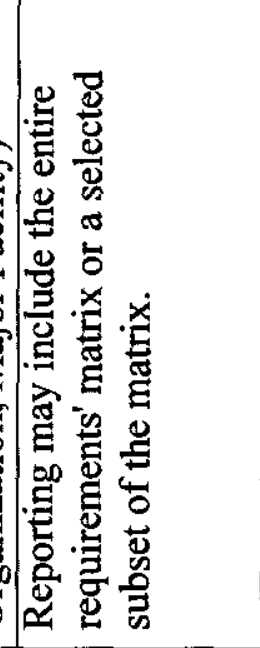 & 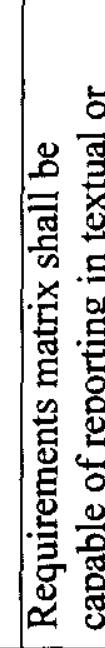 \\
\hline 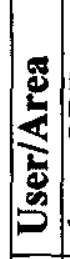 & 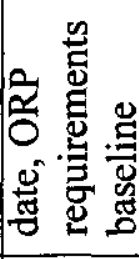 & 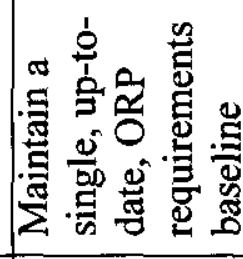 & 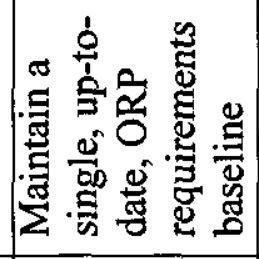 & 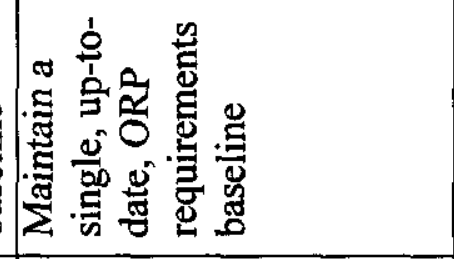 & 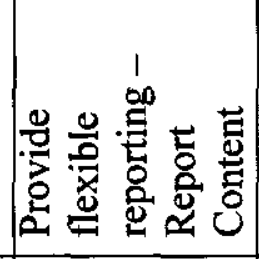 & 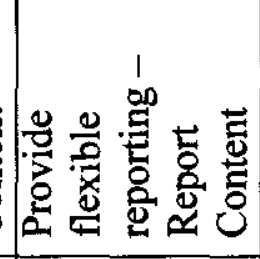 & $\frac{\pi}{0}$ \\
\hline$\stackrel{\theta}{\mathbf{z}}$ & & יָ & $\hat{\mathrm{m}}$ & $\underset{m}{\infty}$ & Im & Po & $F$ \\
\hline
\end{tabular}


RPP-6251 REV. 0

\begin{tabular}{|c|c|c|c|c|c|c|}
\hline $\mid$ & & $\stackrel{\infty}{\infty}$ & $\stackrel{s}{\infty}$ & $y_{2}$ & $\nu_{\nu}^{\infty}$ & $\ddot{\infty}$ \\
\hline 惑 & & 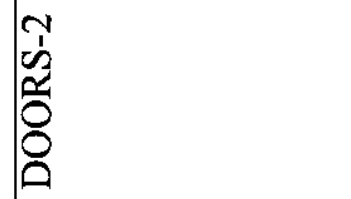 & 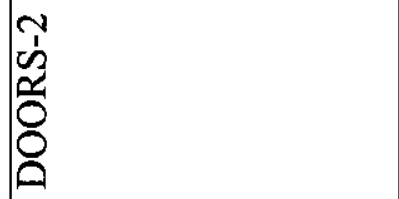 & 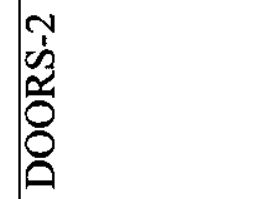 & $\mathbb{z}$ & 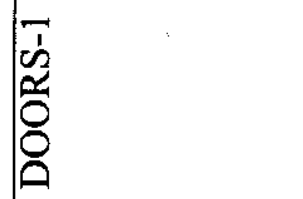 \\
\hline 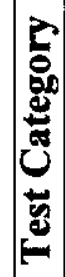 & & 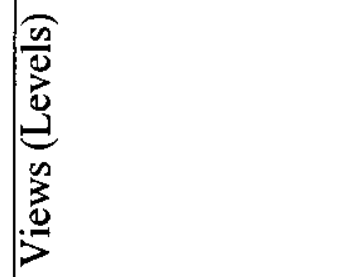 & $\begin{array}{l}\text { 동 } \\
\text { 足 } \\
\text { x }\end{array}$ & 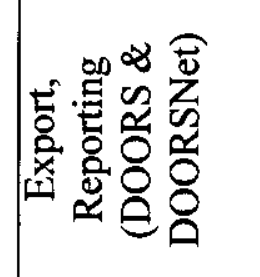 & 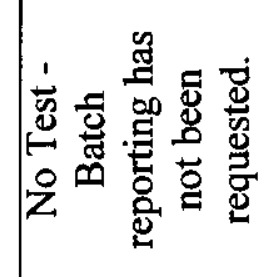 & 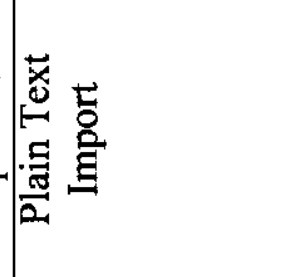 \\
\hline 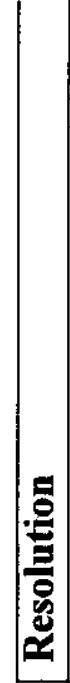 & & 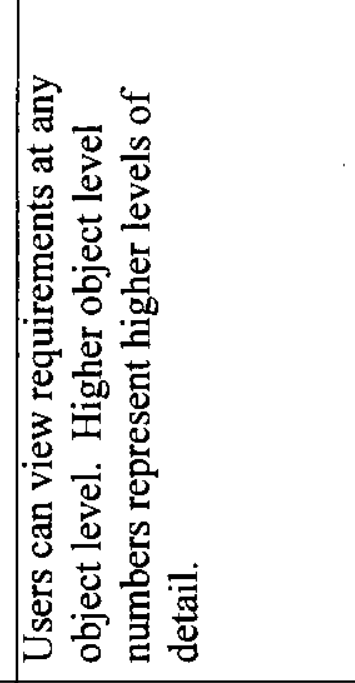 & 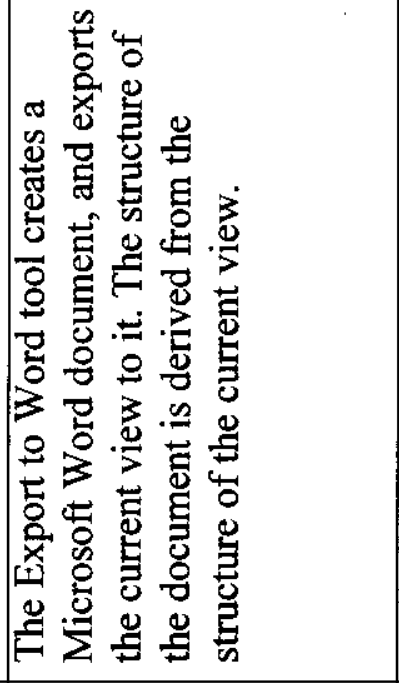 & 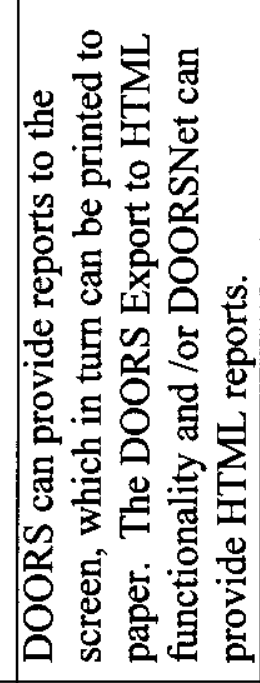 & 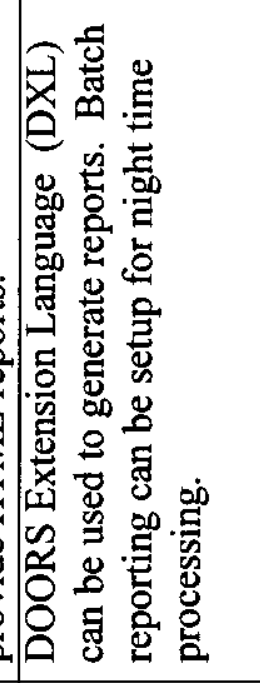 & 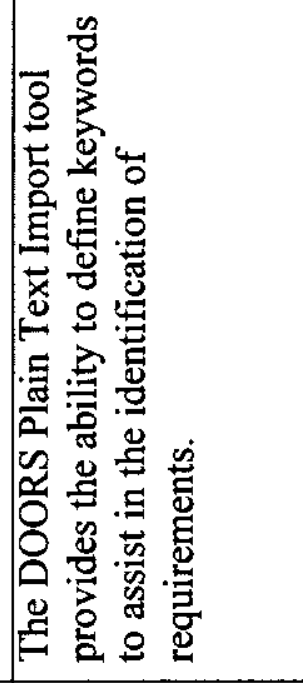 \\
\hline 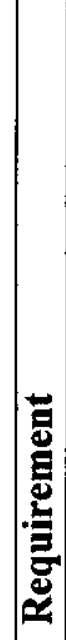 & 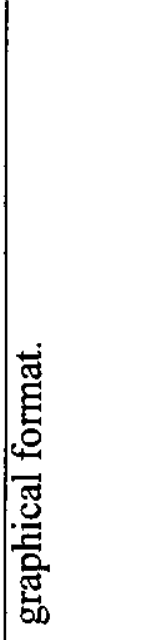 & 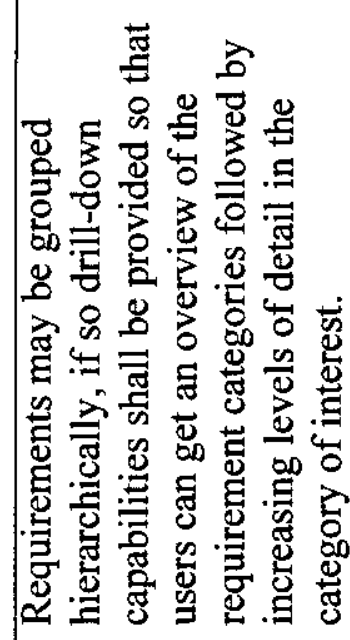 & 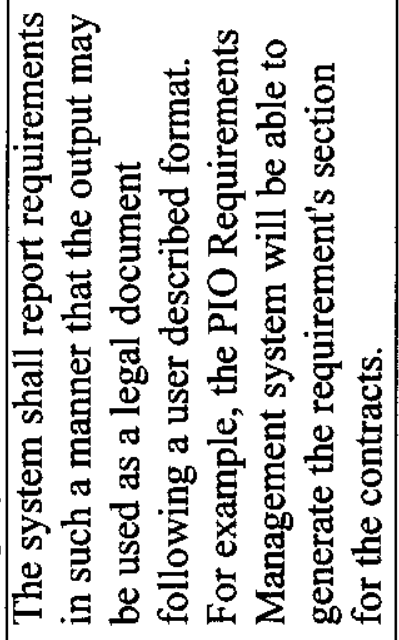 & 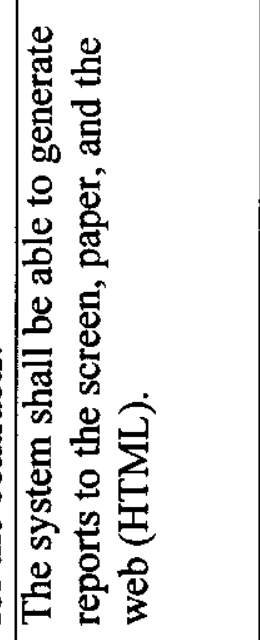 & 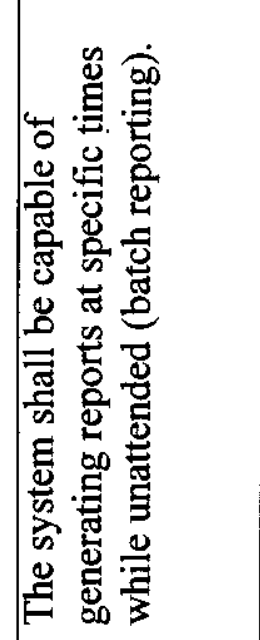 & 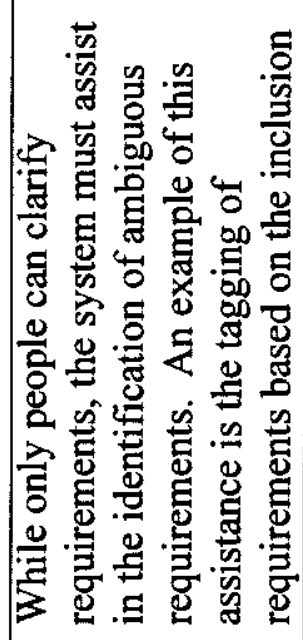 \\
\hline 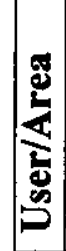 & 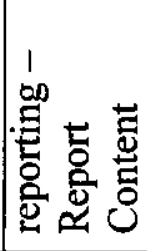 & 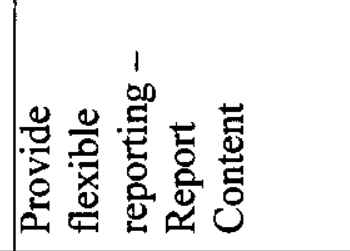 & 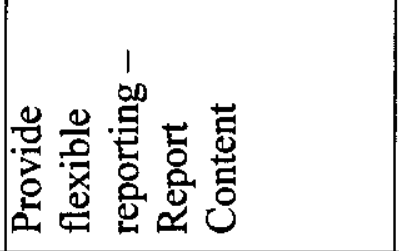 & 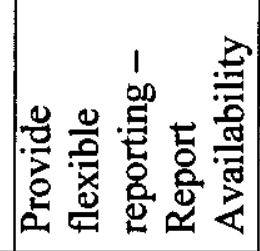 & 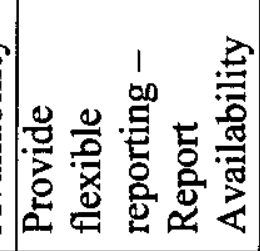 & 总 \\
\hline $\mathbf{z}$ & & $\mathcal{F}$ & $F$ & 扵 & $\mathscr{f}$ & I \\
\hline
\end{tabular}


RPP-6251 REV. 0

\begin{tabular}{|c|c|c|c|c|c|c|c|}
\hline 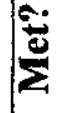 & & $\stackrel{\infty}{\infty}$ & $\ddot{\infty}$ & $\stackrel{\infty}{\varnothing}$ & $\stackrel{\infty}{\infty}$ & 2 & $x^{\infty}$ \\
\hline 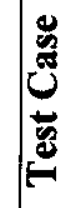 & & 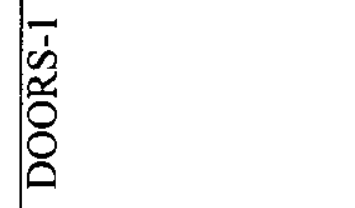 & 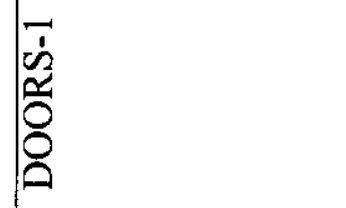 & 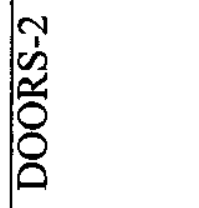 & $\mathbb{Z}$ & 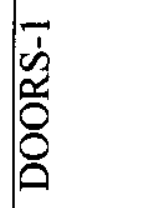 & 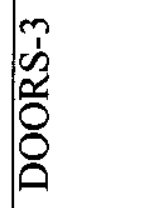 \\
\hline 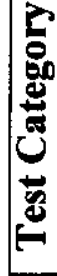 & & 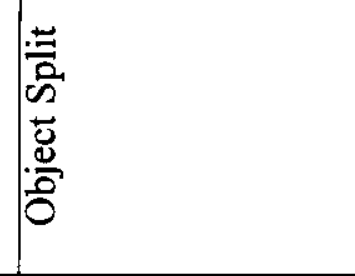 & 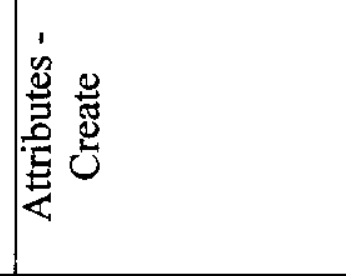 & 鹿 & 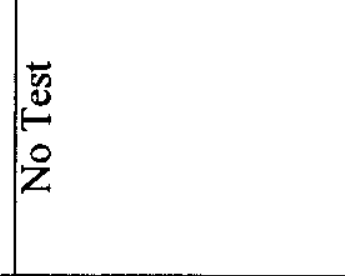 & 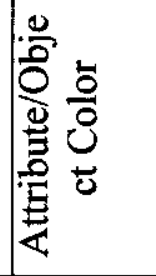 & 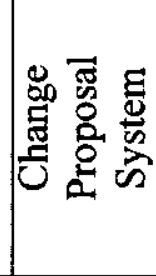 \\
\hline 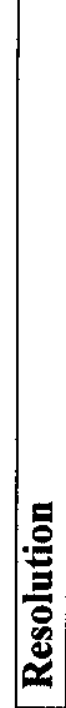 & & 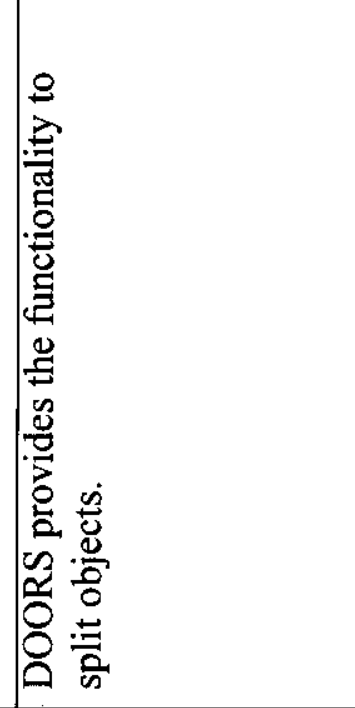 & 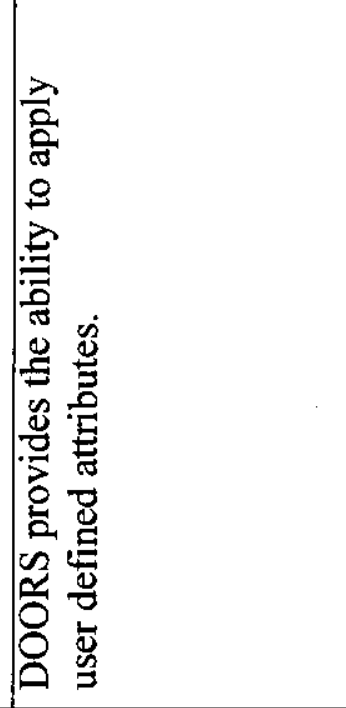 & 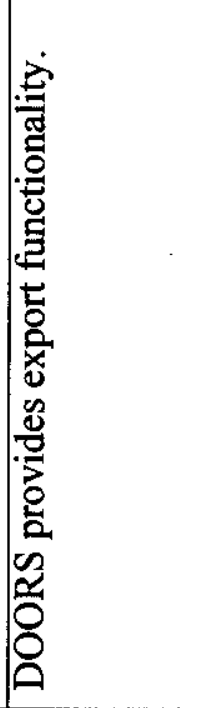 & 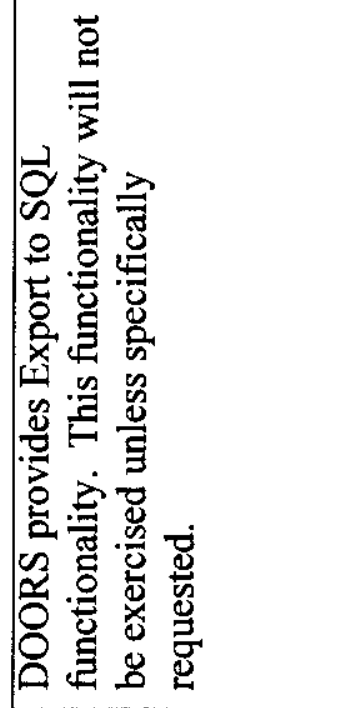 & 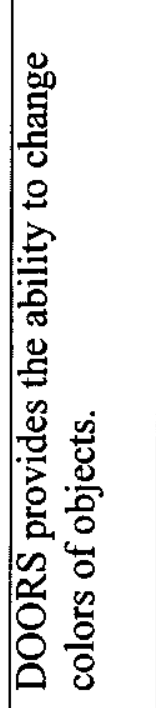 & 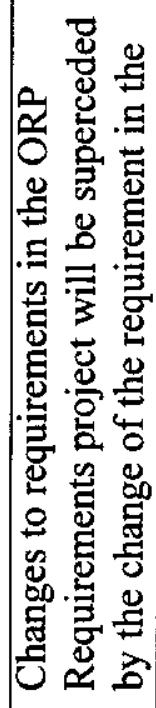 \\
\hline 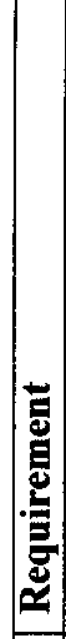 & 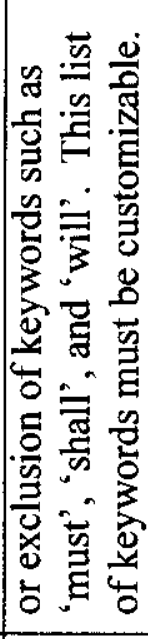 & 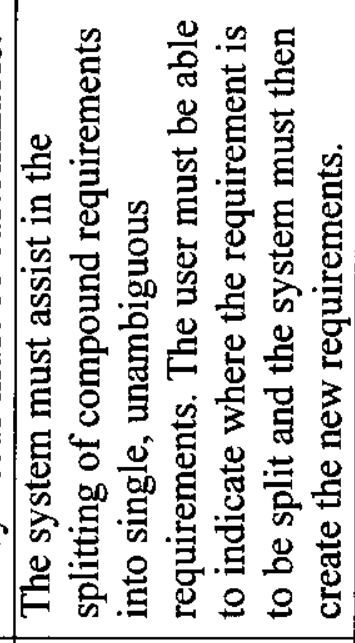 & 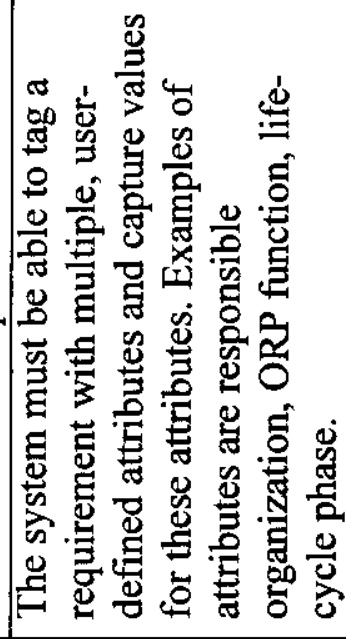 & 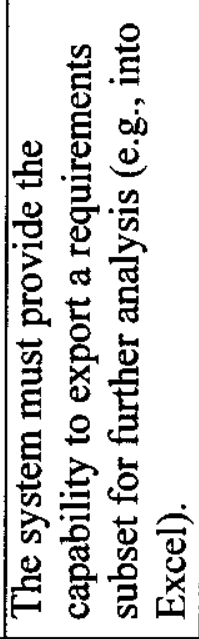 & 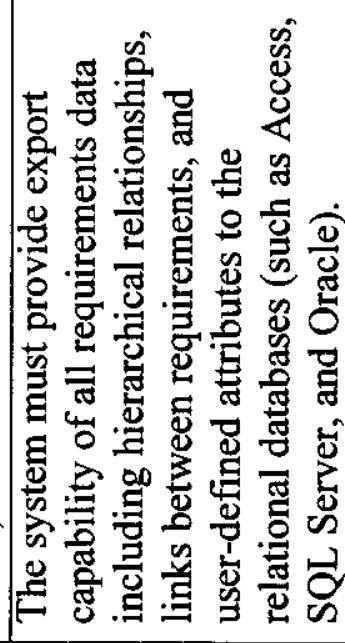 & 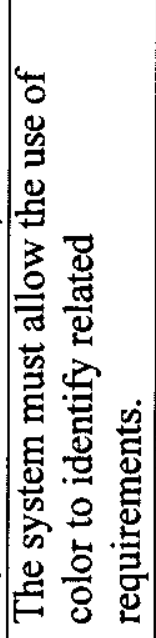 & 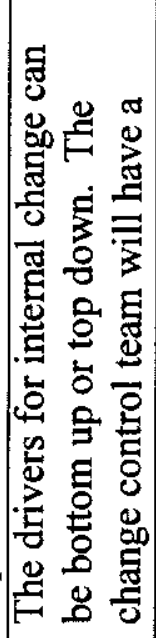 \\
\hline 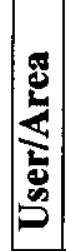 & & 竞 & 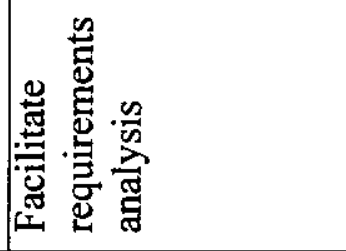 & 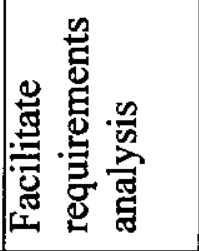 & 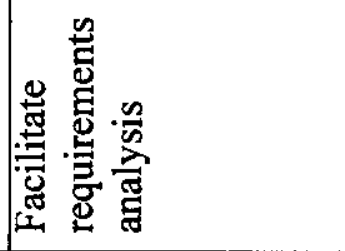 & 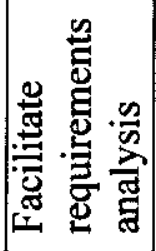 & 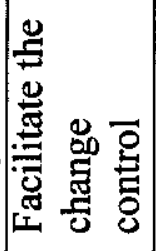 \\
\hline $\mathbf{z}$ & & F & $\$ \infty$ & 朵 & in & $\sqrt{n}$ & in \\
\hline
\end{tabular}


RPP-6251 REV. 0

\begin{tabular}{|c|c|c|c|c|c|}
\hline $\mid$ & & $x^{\infty}$ & $\nu^{\infty}$ & $\stackrel{e}{\infty}$ & $\nu^{\infty}$ \\
\hline 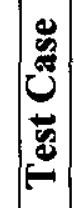 & & $\begin{array}{l}n \\
\tilde{2} \\
\tilde{2} \\
0 \\
0 \\
0\end{array}$ & $\begin{array}{l}\tilde{y} \\
\tilde{n} \\
\tilde{\delta} \\
\delta \\
\delta \\
0\end{array}$ & $\begin{array}{l}\hat{n} \\
\hat{2} \\
\delta \\
\delta \\
\delta\end{array}$ & $\begin{array}{l}\tilde{y} \\
\tilde{\alpha} \\
\delta \\
\delta \\
\delta\end{array}$ \\
\hline & & 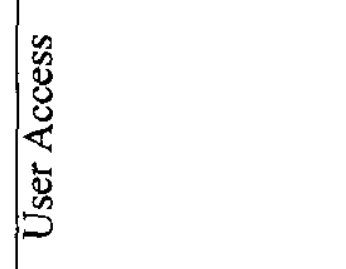 & 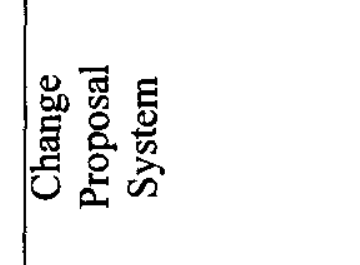 & 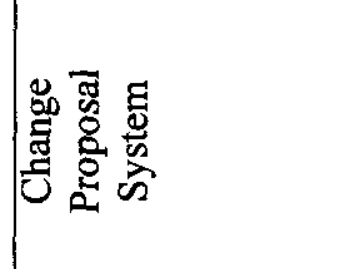 & 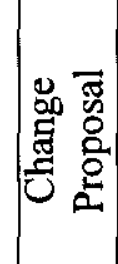 \\
\hline & 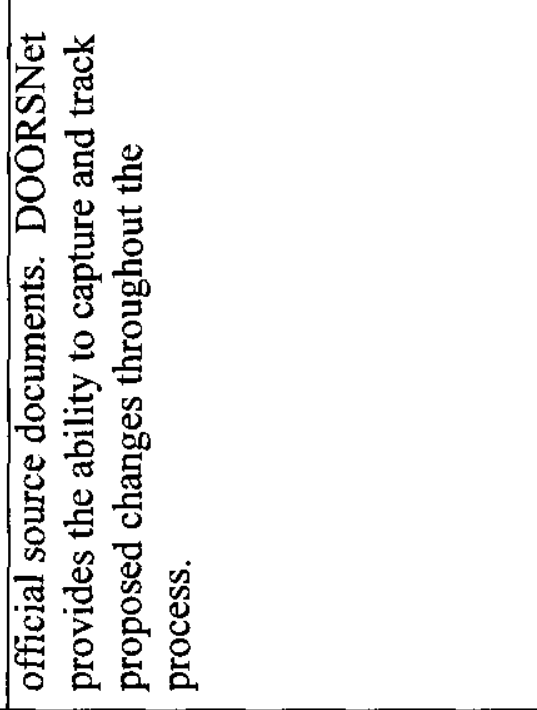 & 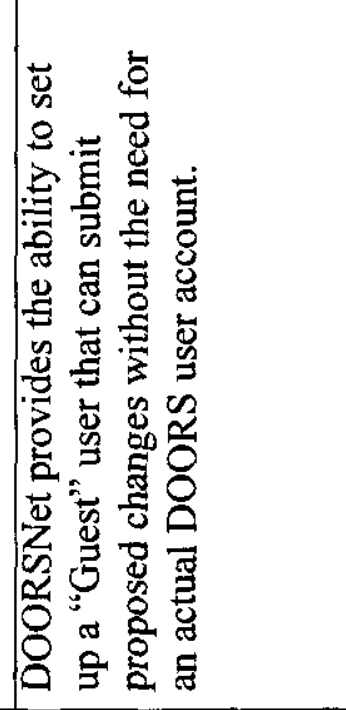 & 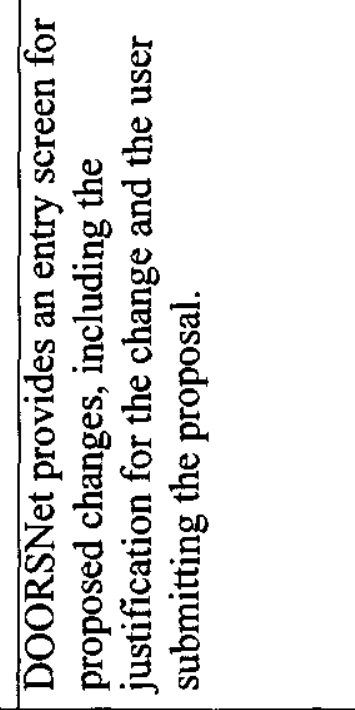 & 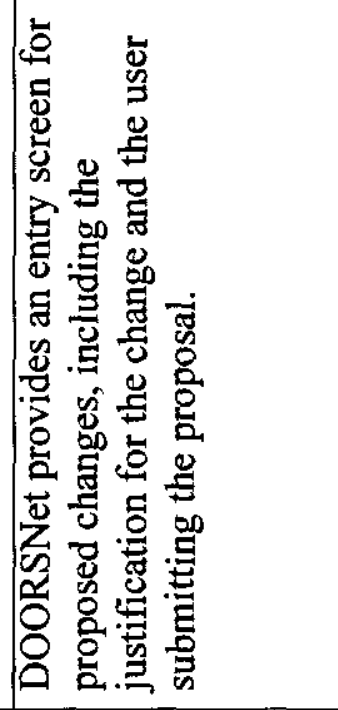 & 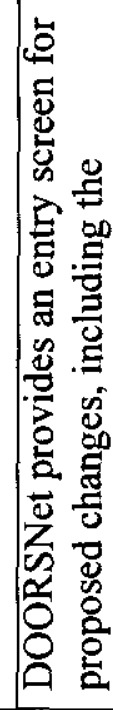 \\
\hline : & 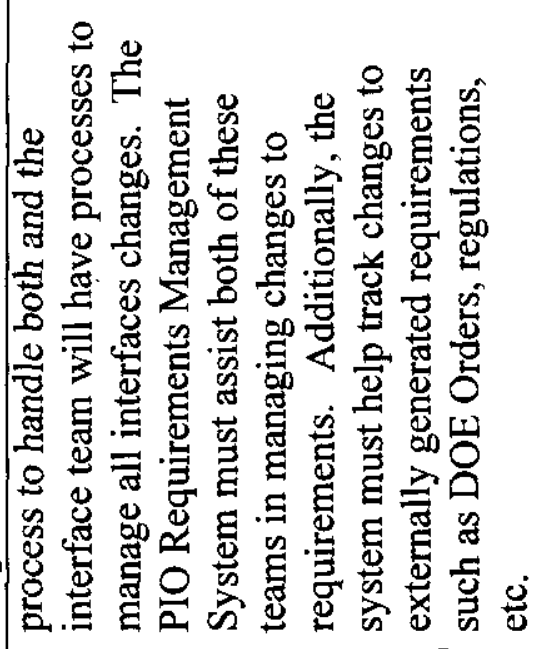 & 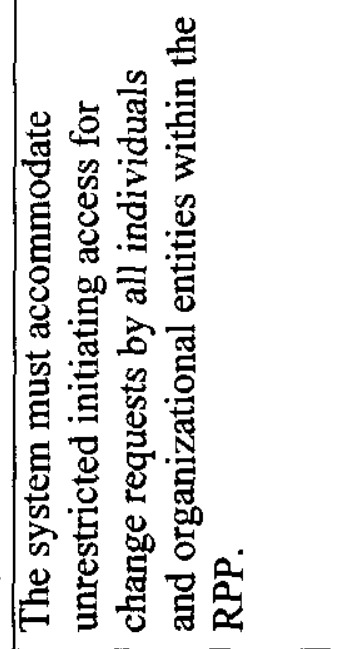 & 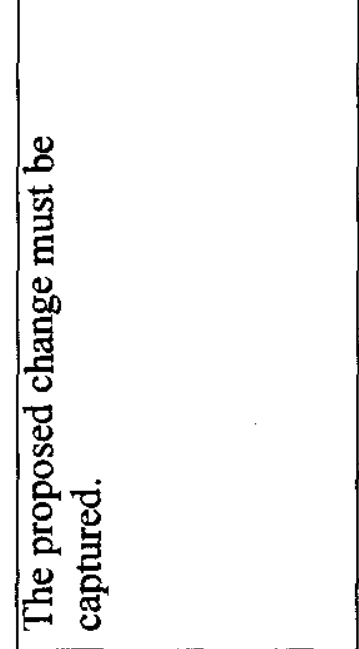 & 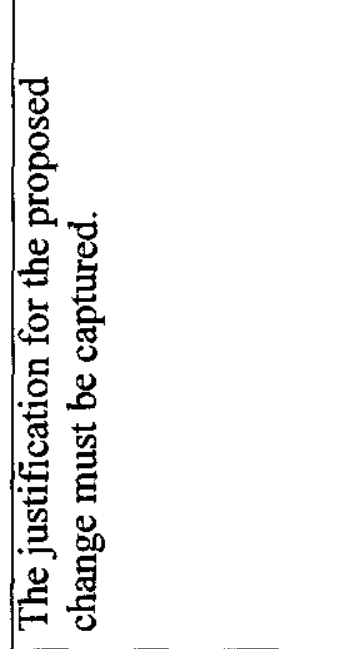 & 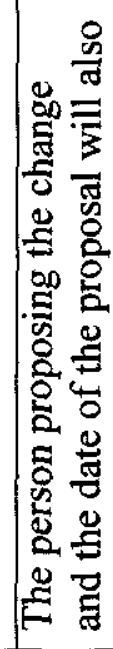 \\
\hline 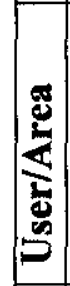 & 萢 & 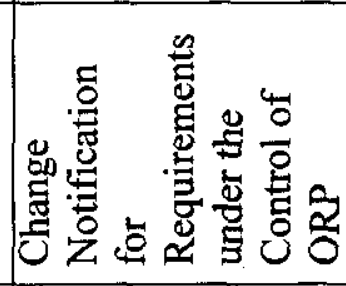 & 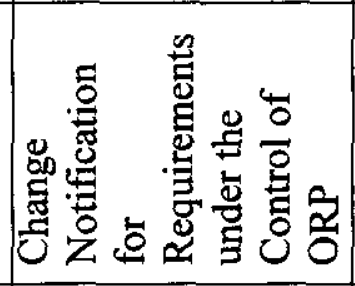 & 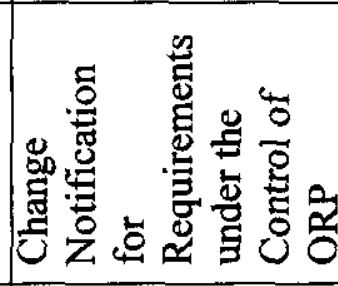 & 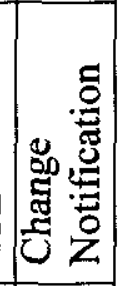 \\
\hline 2 & & in & 借 & $\approx$ & in \\
\hline
\end{tabular}


RPP-6251 REV. 0

\begin{tabular}{|c|c|c|c|c|}
\hline $\mid$ & & $\stackrel{\infty}{\nu}$ & $\sum_{\nu}^{\infty}$ & $\ddot{\infty}$ \\
\hline 惫 & & 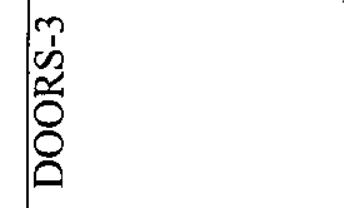 & 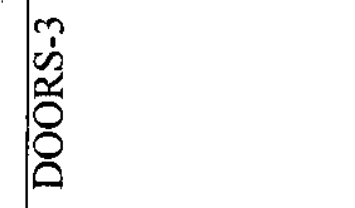 & $\mathbb{z}$ \\
\hline 空 & 焉 & 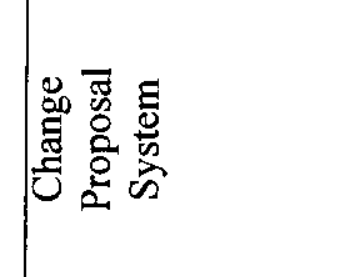 & 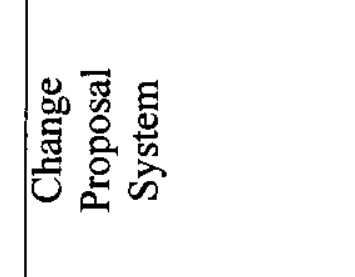 & 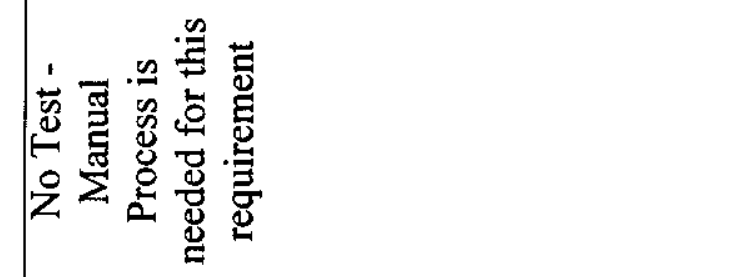 \\
\hline & 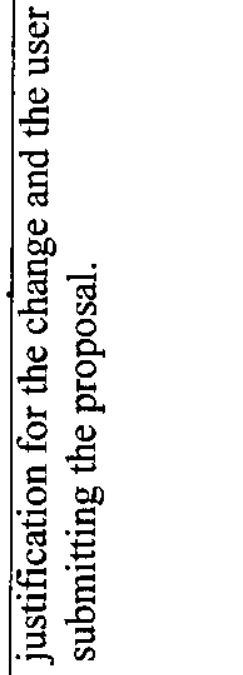 & 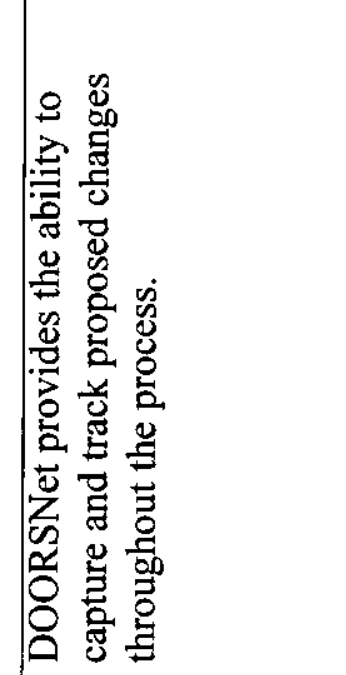 & 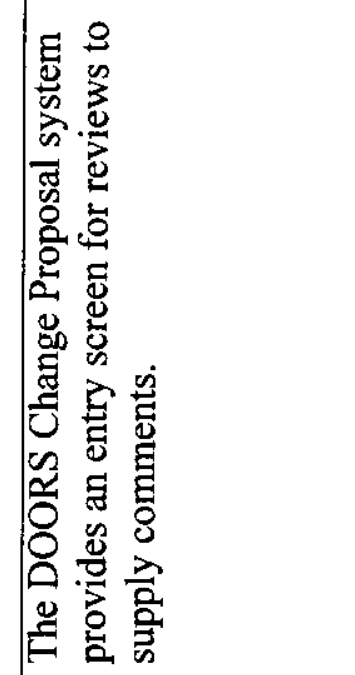 & 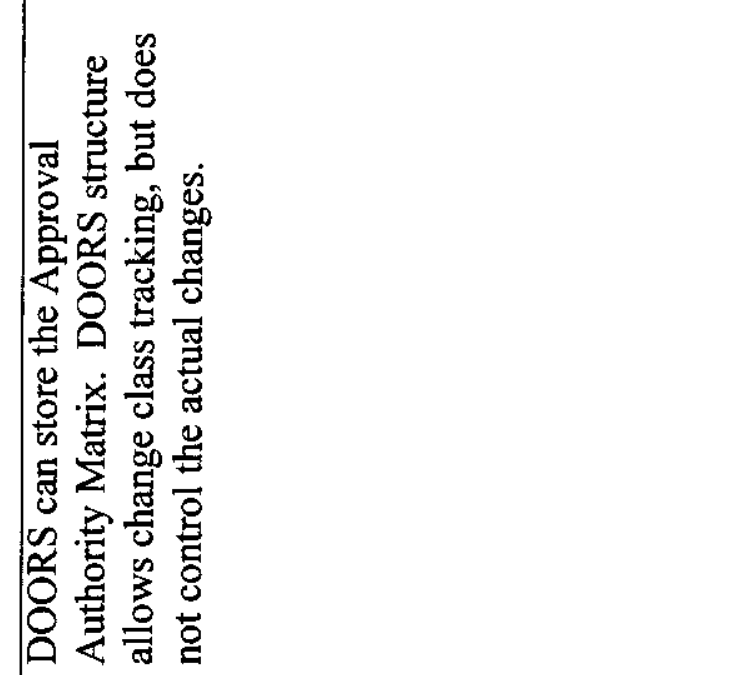 \\
\hline 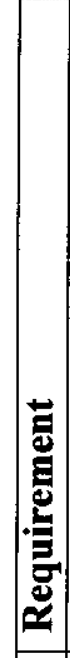 & & 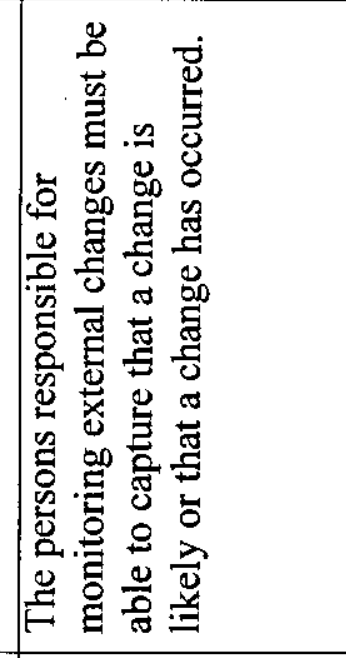 & 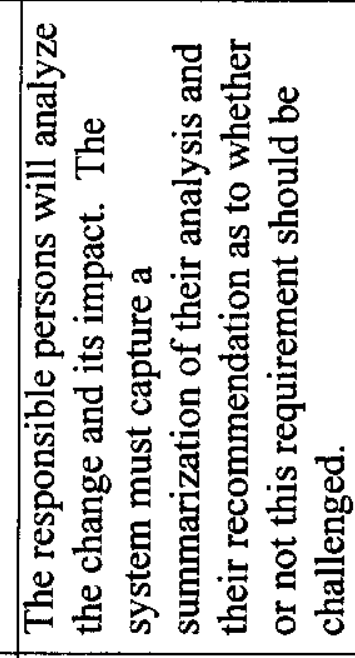 & 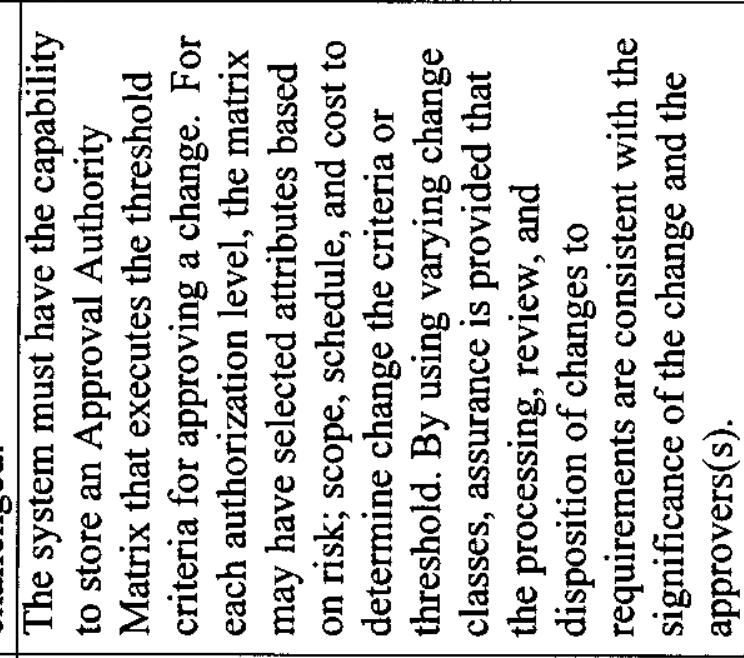 \\
\hline 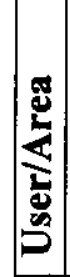 & 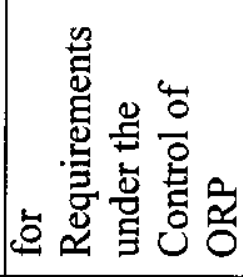 & 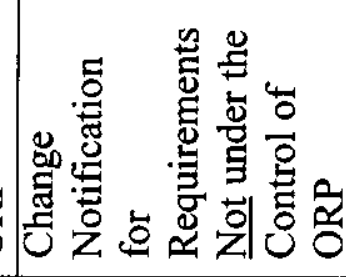 & 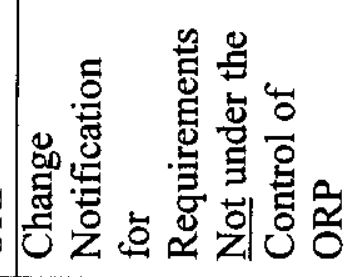 & 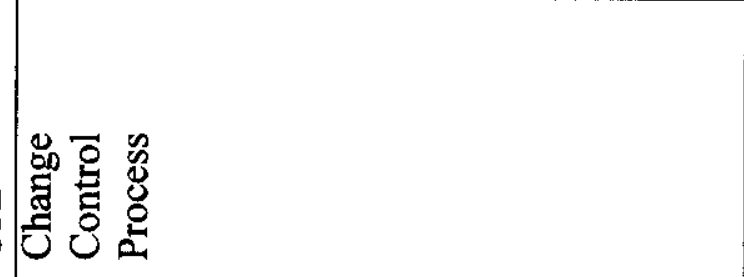 \\
\hline$\dot{z}$ & & in & $\infty$ & in \\
\hline
\end{tabular}


RPP-6251 REV. 0

\begin{tabular}{|c|c|c|c|c|c|c|}
\hline 害 & $\stackrel{\infty}{\infty}$ & $\stackrel{0}{\nu}$ & zo & $\frac{\infty}{2}$ & $\stackrel{\infty}{\infty}$ & 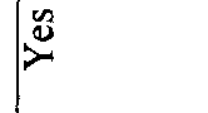 \\
\hline & 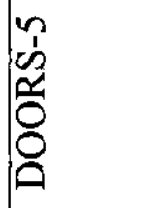 & 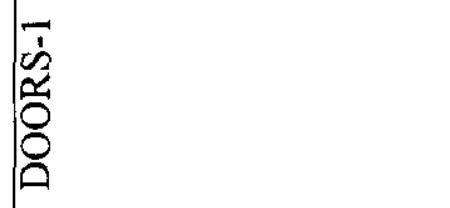 & 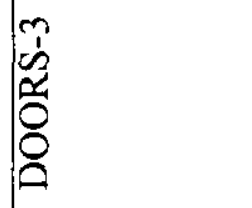 & $\begin{array}{l}\tilde{N} \\
\tilde{2} \\
\delta \\
\delta \\
\delta \\
\delta\end{array}$ & $\begin{array}{l}\hat{n} \\
\hat{n} \\
\tilde{z} \\
\delta \\
\delta \\
0\end{array}$ & 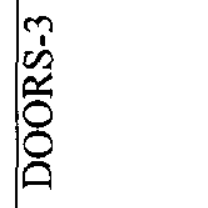 \\
\hline & 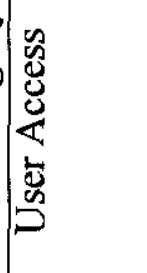 & 曾 & 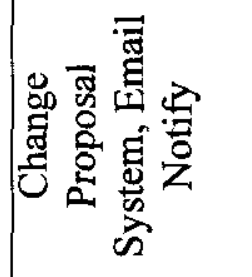 & 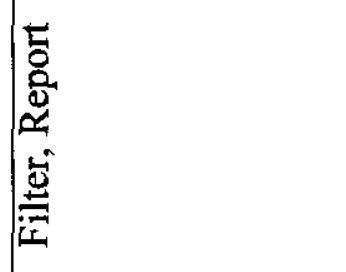 & 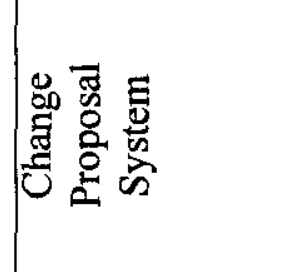 & 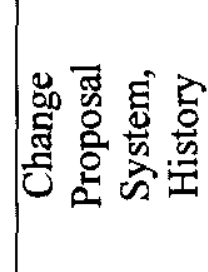 \\
\hline & 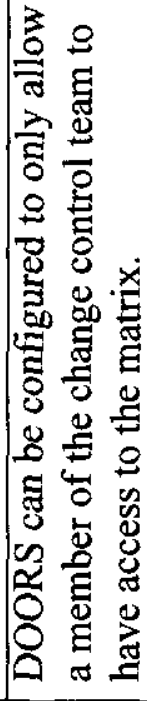 & 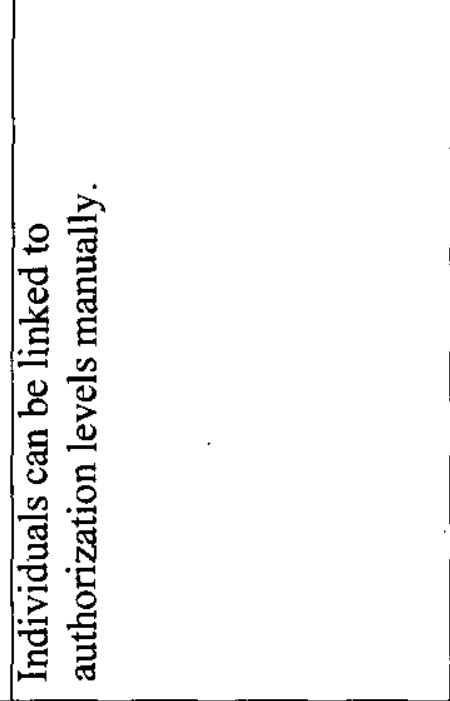 & 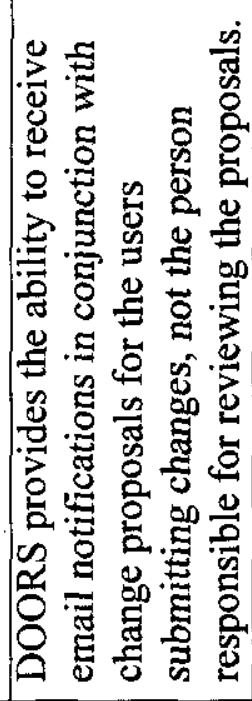 & 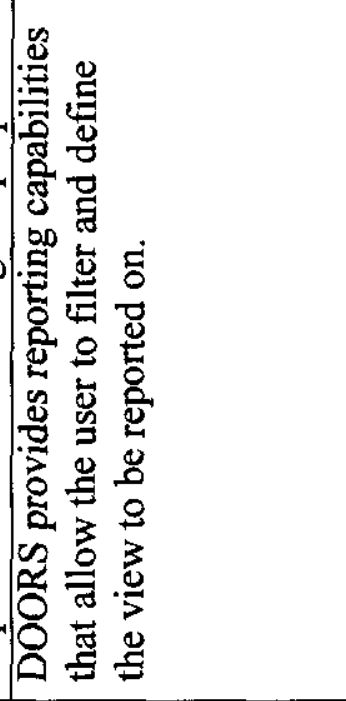 & 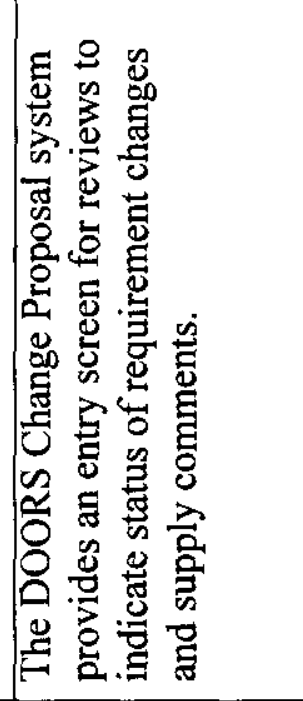 & 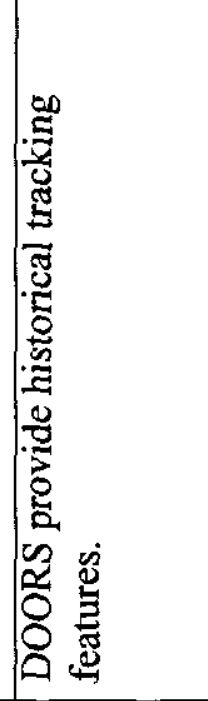 \\
\hline 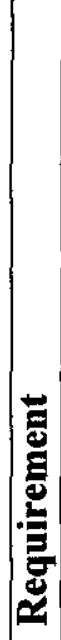 & 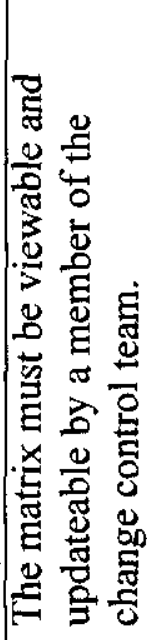 & 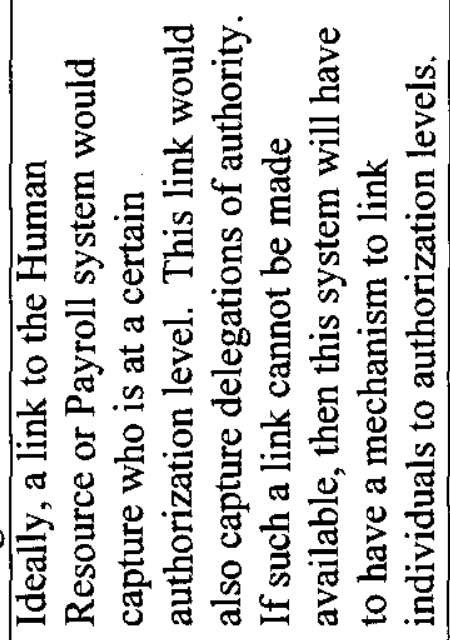 & 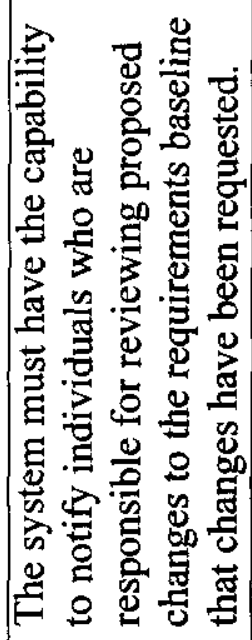 & 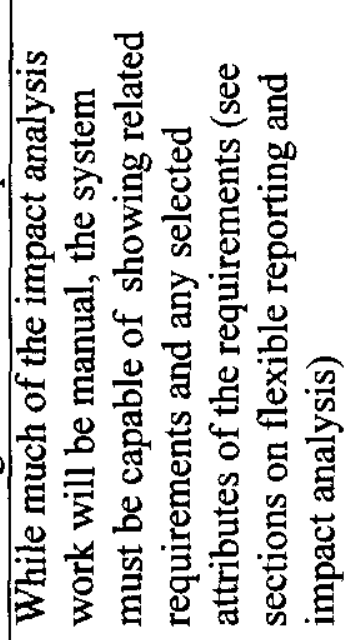 & 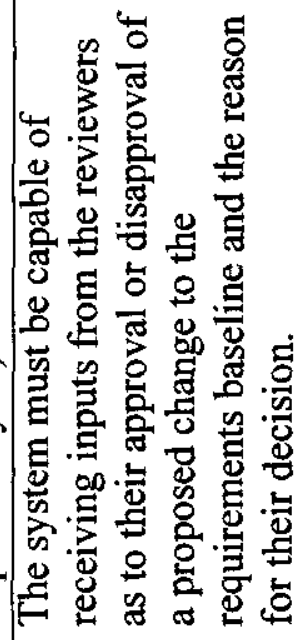 & 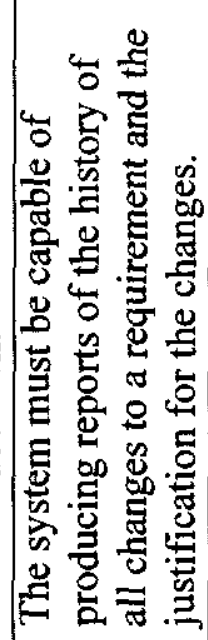 \\
\hline 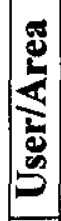 & 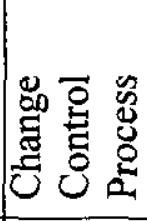 & 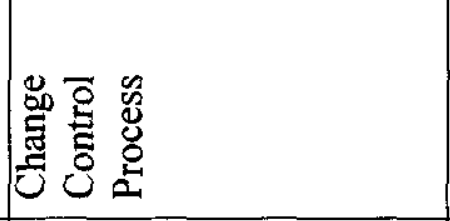 & 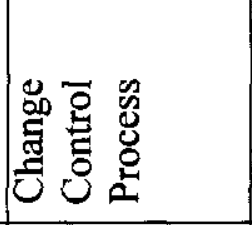 & 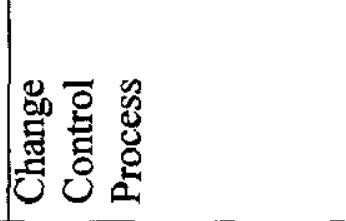 & 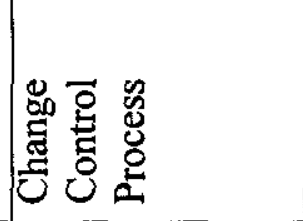 & 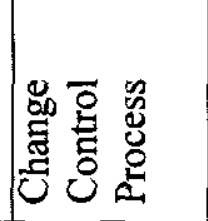 \\
\hline $\mathbf{z}$ & & $\sqrt{6}$ & $\widehat{్}$ & 10 & 估 & 10 \\
\hline
\end{tabular}


RPP-6251 REV. 0

\begin{tabular}{|c|c|c|c|c|c|c|c|c|}
\hline 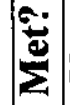 & $\stackrel{\mathscr{s}}{\nu}$ & $\stackrel{\otimes}{\infty}$ & $\ddot{\infty}$ & $\sum^{\infty}$ & $\stackrel{s}{\nu}$ & 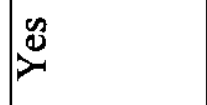 & $\mathscr{\infty}_{-1}^{\infty}$ & $\stackrel{\infty}{\infty}$ \\
\hline & & 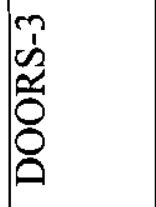 & 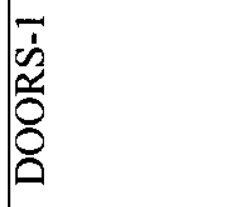 & 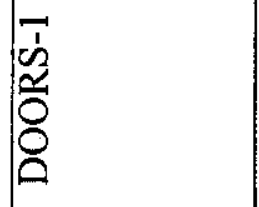 & $\begin{array}{l}\overline{1} \\
\ddot{2} \\
8 \\
8 \\
0\end{array}$ & 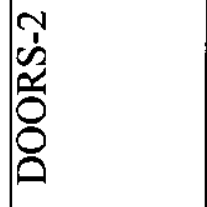 & 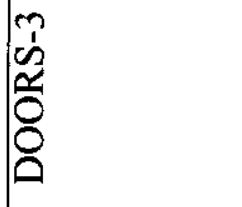 & $\mathbb{Z}$ \\
\hline & 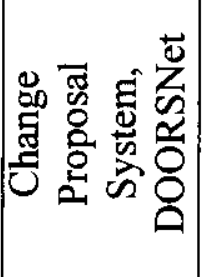 & 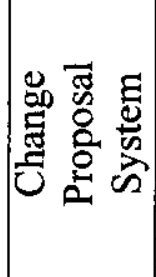 & 总 & 考 & 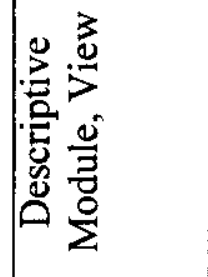 & 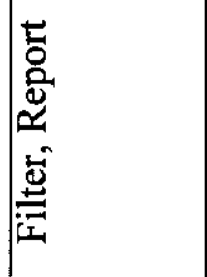 & 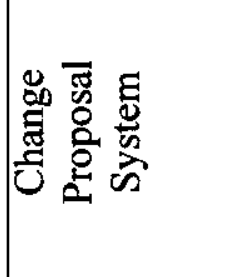 & 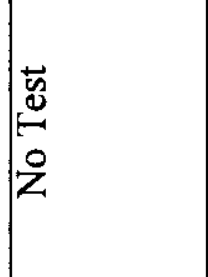 \\
\hline 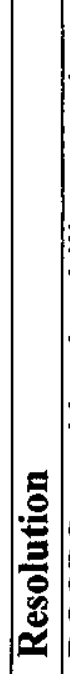 & 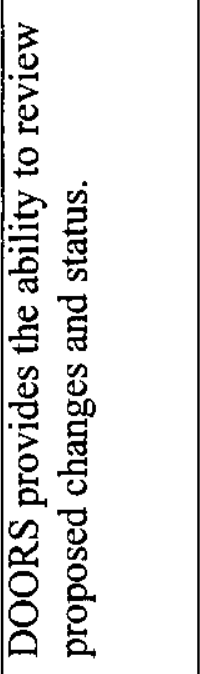 & 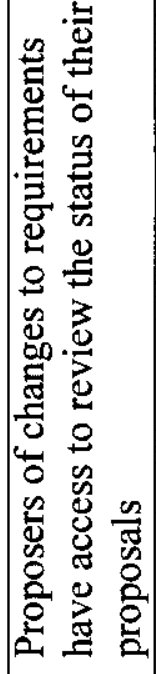 & 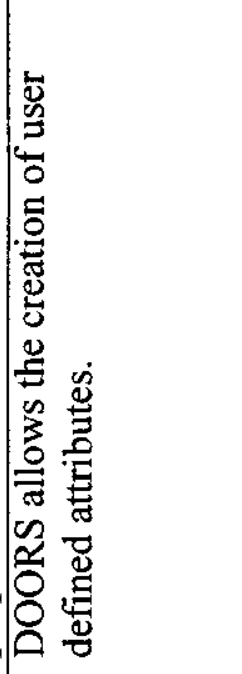 & 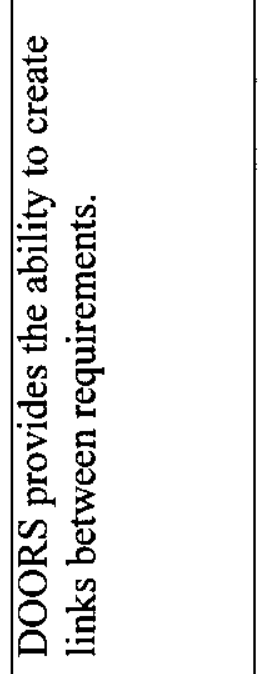 & 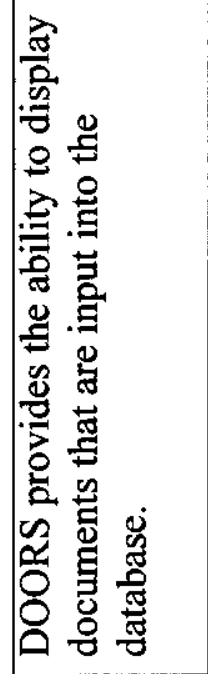 & 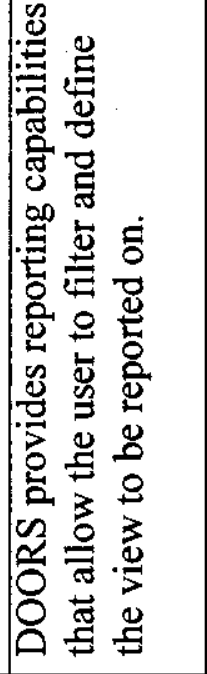 & 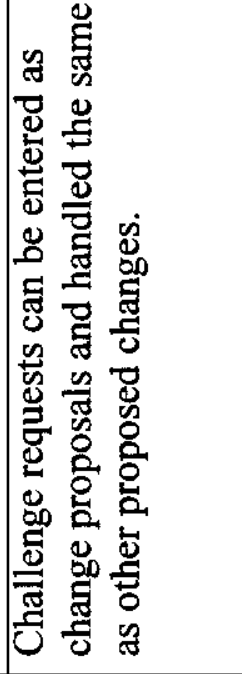 & 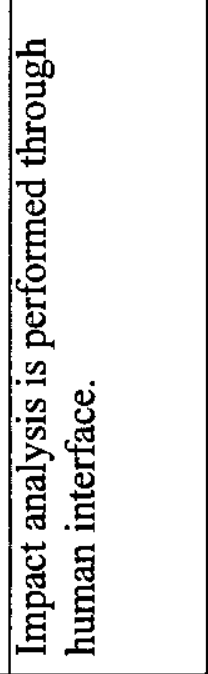 \\
\hline $\mid$ & 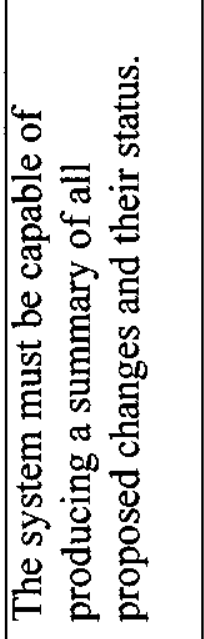 & 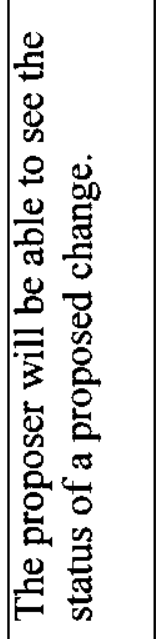 & 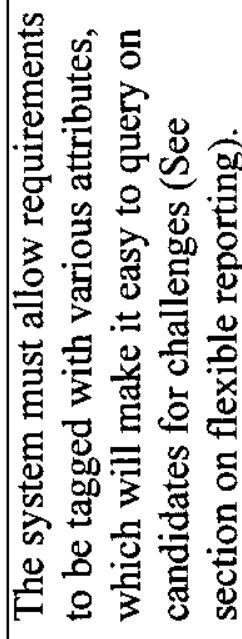 & 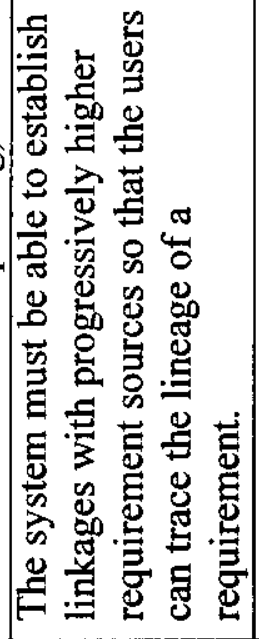 & 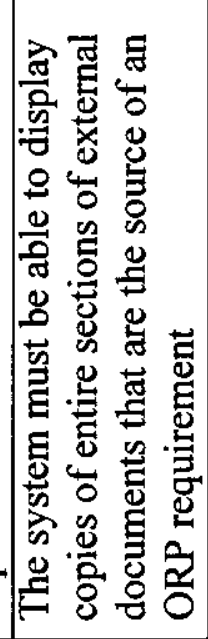 & 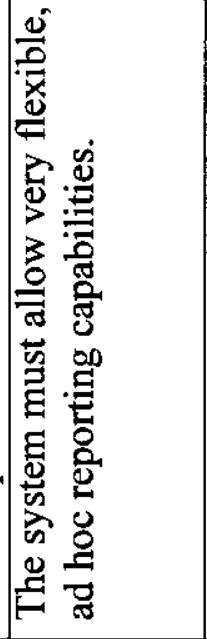 & 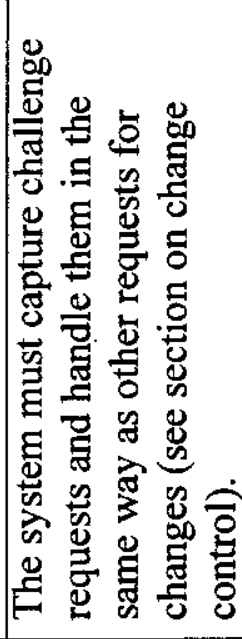 & 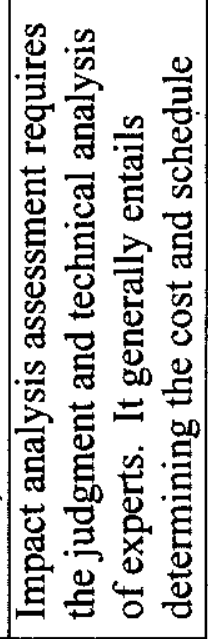 \\
\hline 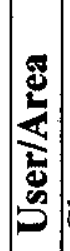 & 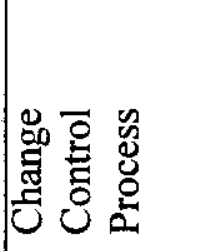 & 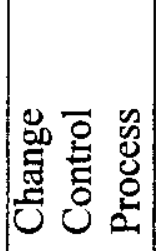 & 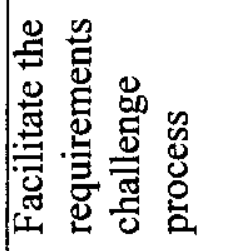 & 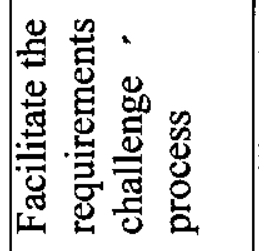 & 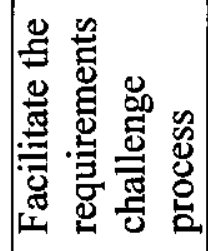 & 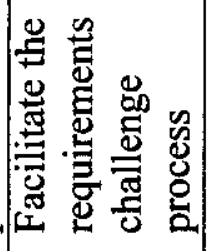 & 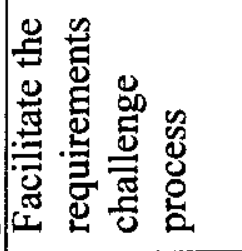 & 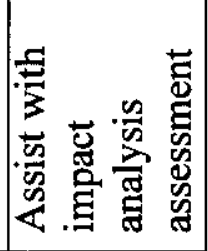 \\
\hline 2 & 8 & $\widehat{6}$ & 100 & 80 & $R$ & $F$ & $\mathbb{N}$ & 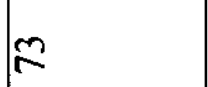 \\
\hline
\end{tabular}


RPP-6251 REV. 0

\begin{tabular}{|c|c|c|c|c|c|c|c|c|}
\hline 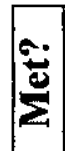 & & $\sum_{\substack{\infty \\
\infty}}^{\infty}$ & $\int_{x}^{\infty}$ & $x_{2}^{\infty}$ & $\stackrel{\infty}{\infty}$ & $y^{y}$ & $\overbrace{}^{\infty}$ & $\rightleftharpoons$ \\
\hline$\left|\begin{array}{|c}\mathscr{2} \\
\tilde{z} \\
0\end{array}\right|$ & & 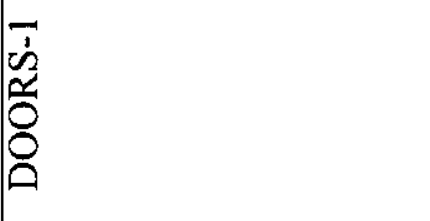 & $\begin{array}{l}\vec{i} \\
\hat{\tilde{O}} \\
8 \\
0\end{array}$ & 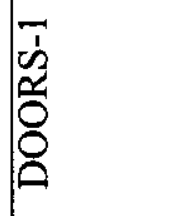 & 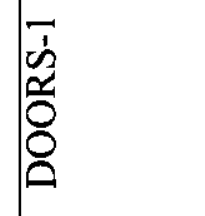 & 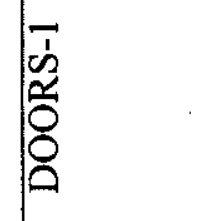 & \begin{tabular}{l}
1 \\
$\tilde{\omega}$ \\
\hdashline \\
0 \\
0 \\
\hdashline
\end{tabular} & 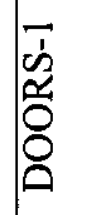 \\
\hline 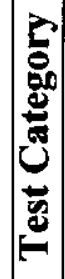 & & 弟 & 道 & 鸢 & 曾 & 当 & 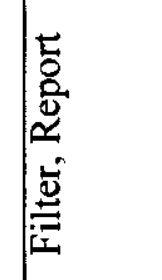 & 营 \\
\hline 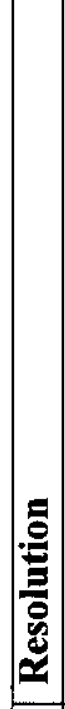 & & 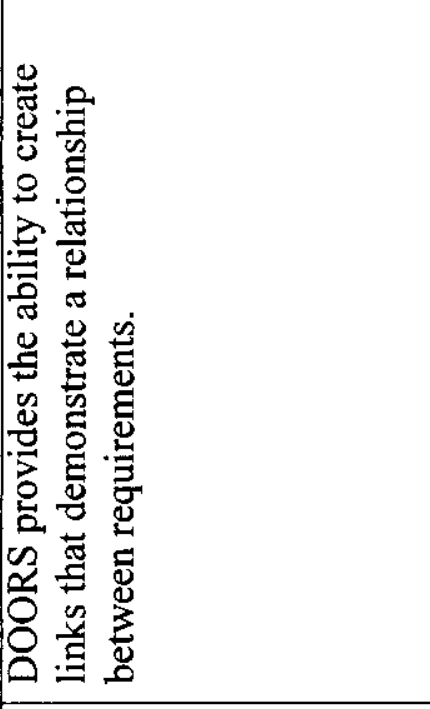 & 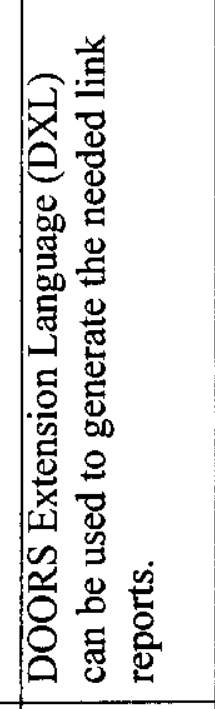 & 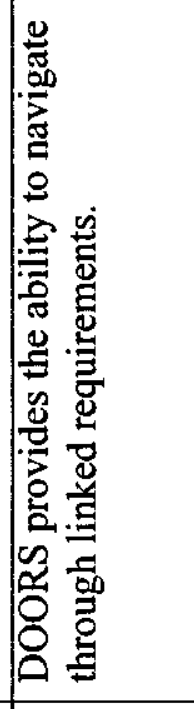 & 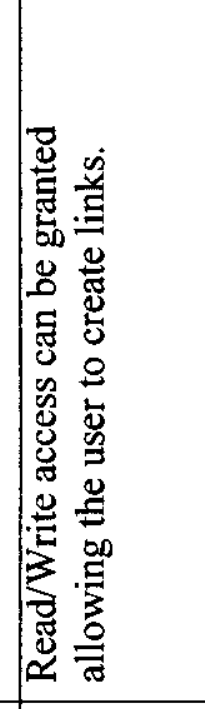 & 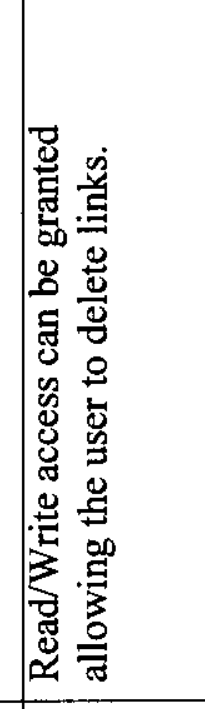 & 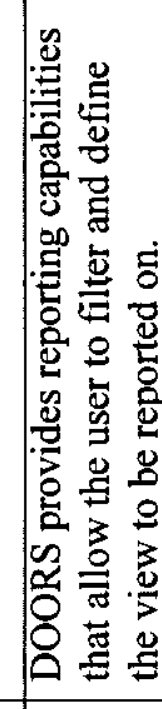 & 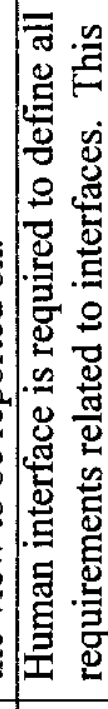 \\
\hline : & 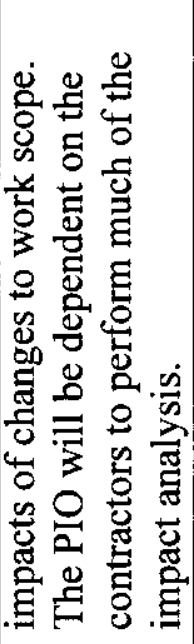 & 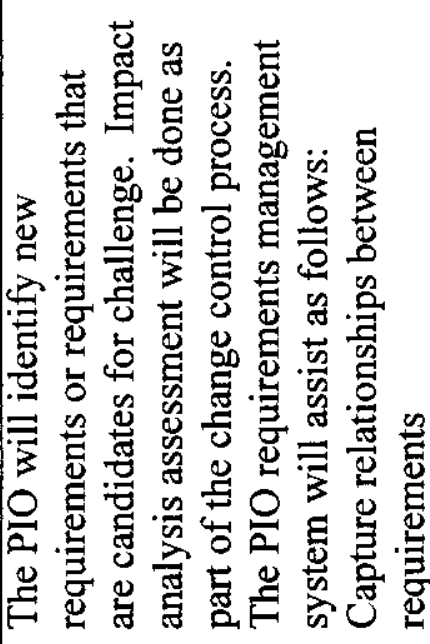 & 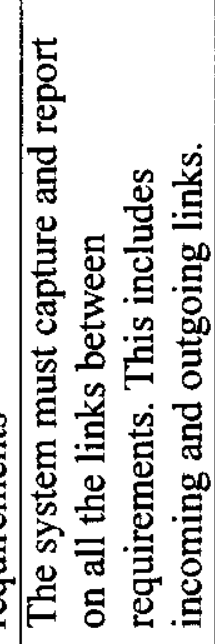 & 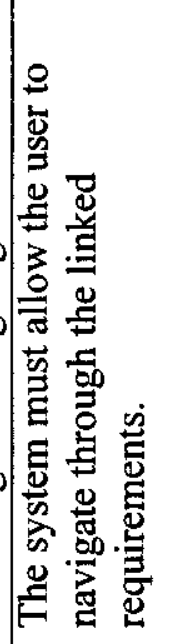 & 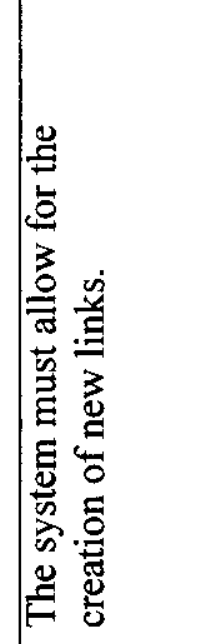 & 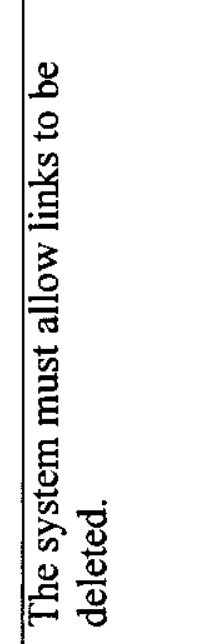 & 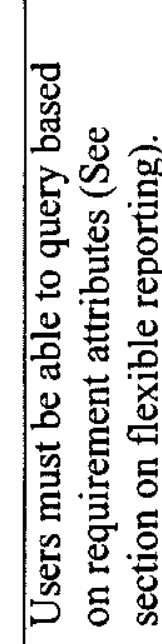 & 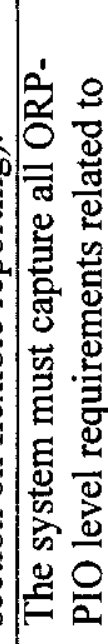 \\
\hline פ্ّ & & 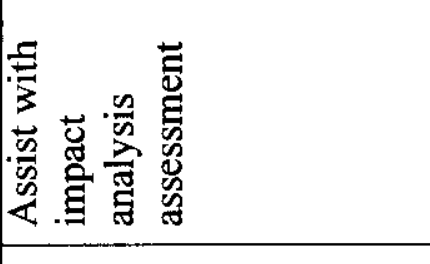 & 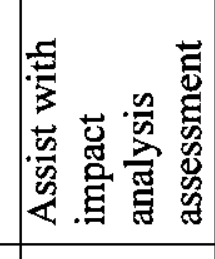 & 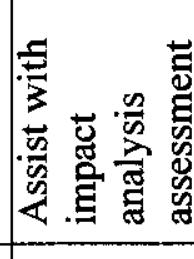 & 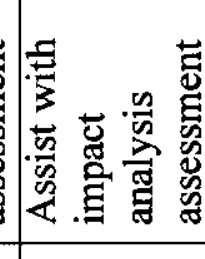 & 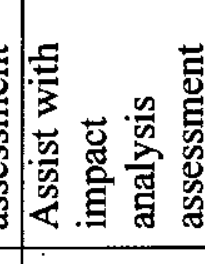 & 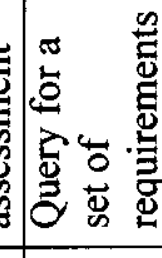 & 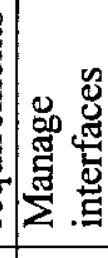 \\
\hline है & & \pm & $\approx$ & 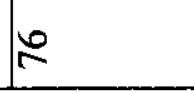 & $E$ & $\infty$ & 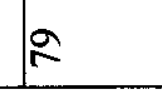 & 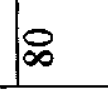 \\
\hline
\end{tabular}


RPP-6251 REV. 0

\begin{tabular}{|c|c|c|c|c|c|c|c|}
\hline $\mid$ & & $\underset{\nu}{\infty}$ & $\underset{\nu}{\infty}$ & $\underbrace{}_{\nu}$ & $\stackrel{\infty}{\infty}$ & 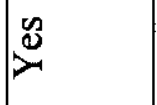 & 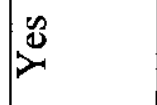 \\
\hline 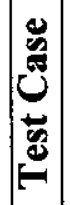 & & 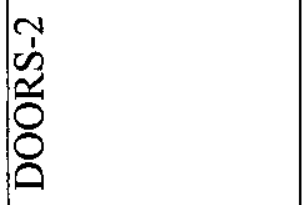 & 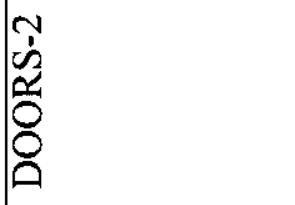 & 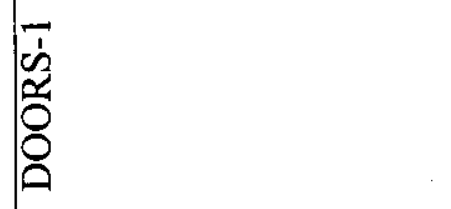 & 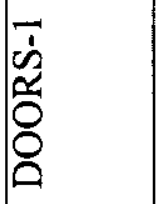 & 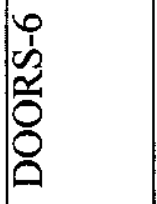 & 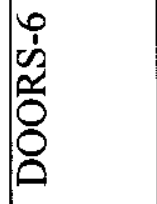 \\
\hline 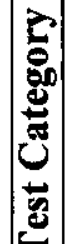 & & 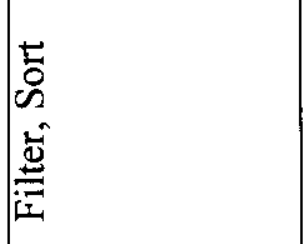 & 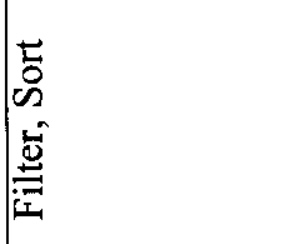 & 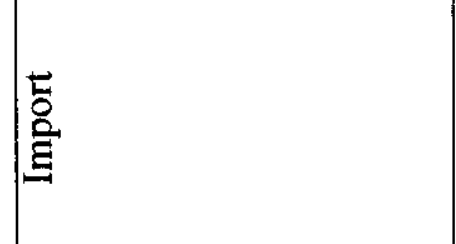 & 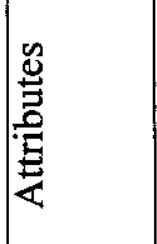 & 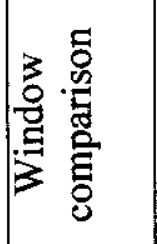 & 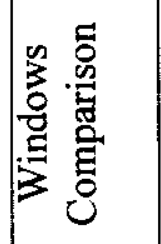 \\
\hline בี & 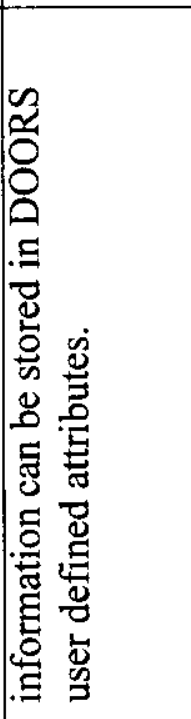 & 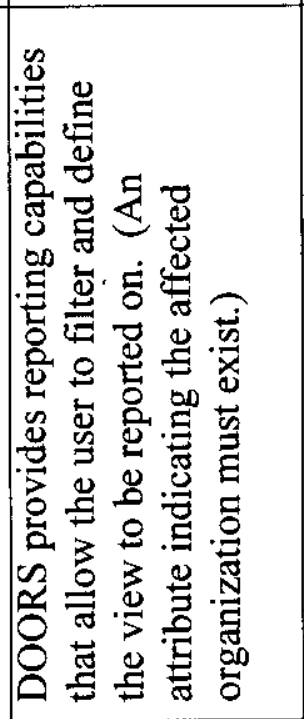 & 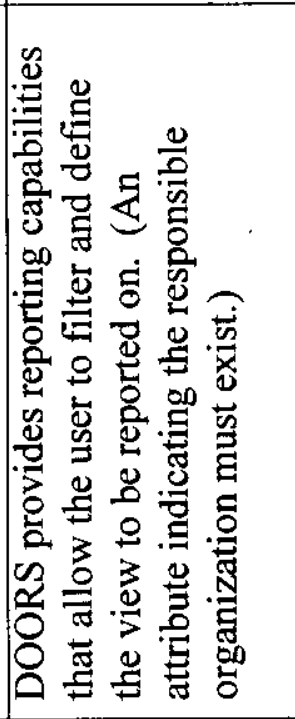 & 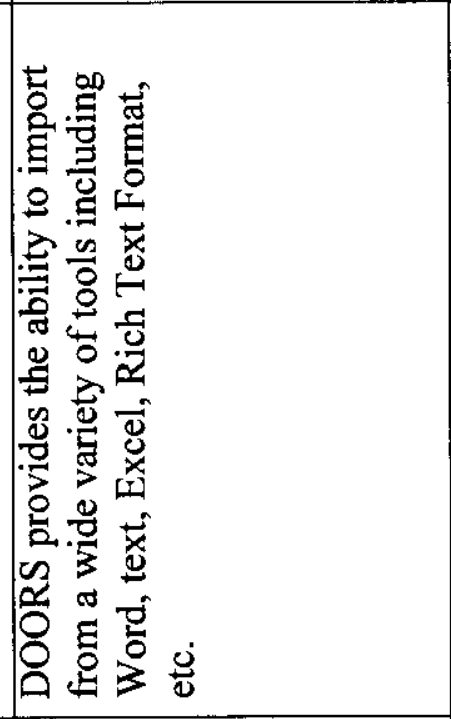 & 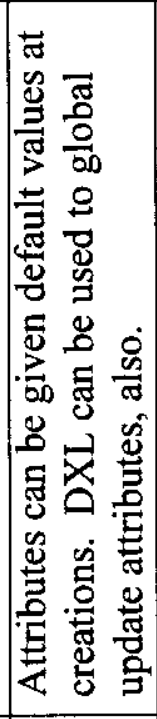 & 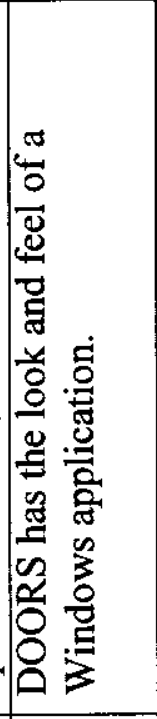 & 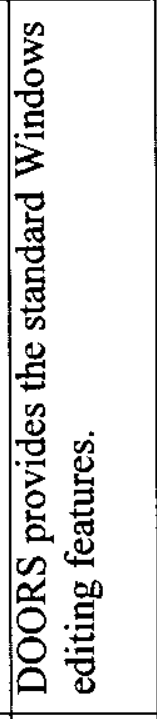 \\
\hline 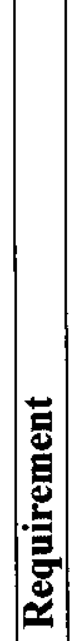 & & 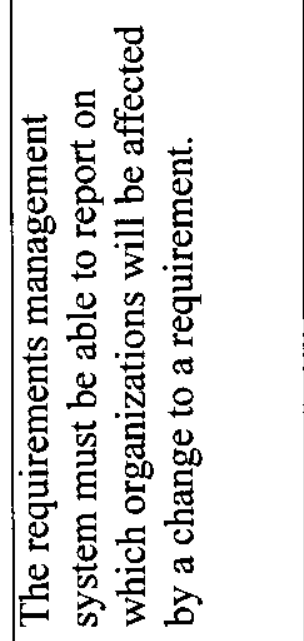 & 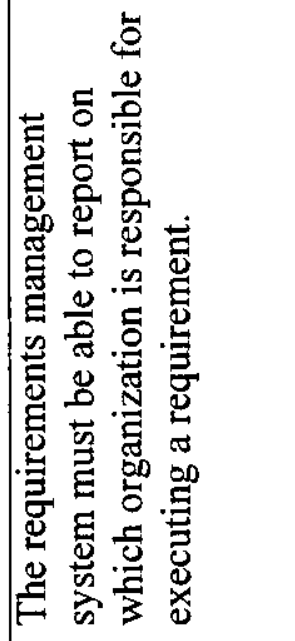 & 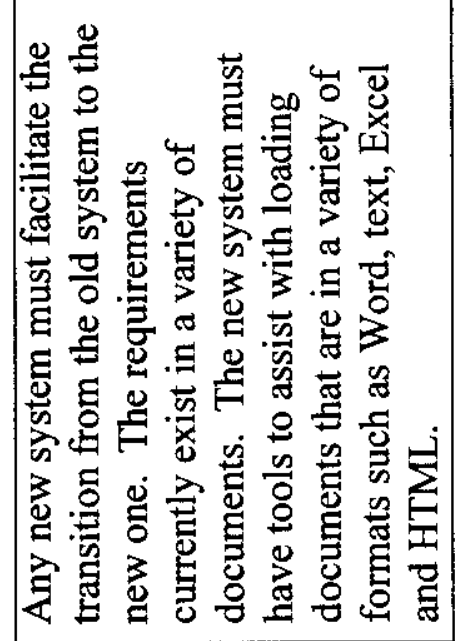 & 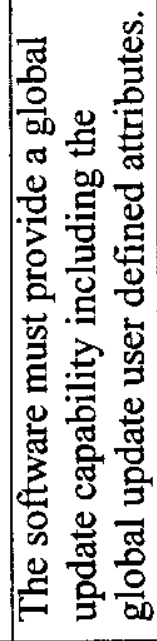 & 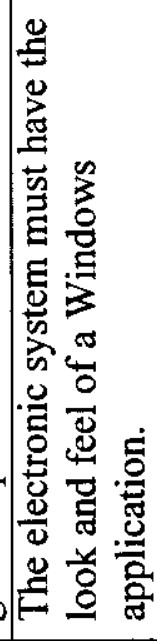 & 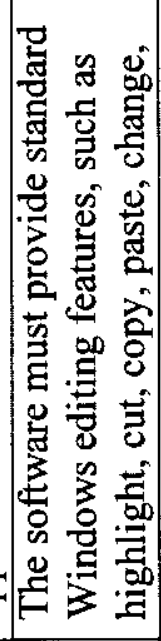 \\
\hline 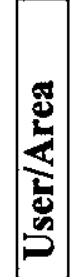 & 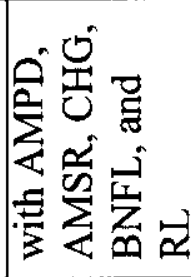 & 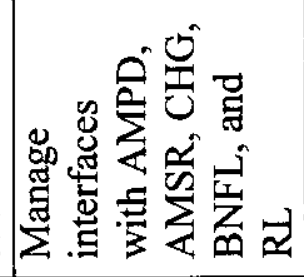 & 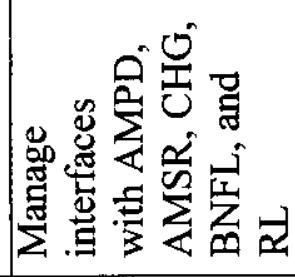 & 莺 & 宽 & 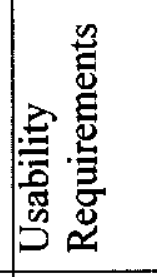 & 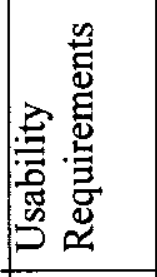 \\
\hline $\mathbf{z}$ & & $\vec{\infty}$ & $\approx$ & $\infty$ & Æ & $\infty$ & $\infty$ \\
\hline
\end{tabular}


RPP-6251 REV. 0

\begin{tabular}{|c|c|c|c|c|c|c|c|c|c|}
\hline$\sum_{\tilde{\Sigma}}^{\tilde{e}}$ & 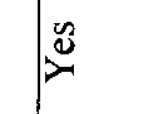 & $\nu^{\infty}$ & $\nu^{\infty}$ & $\overbrace{}^{\infty}$ & $\nu^{\infty}$ & 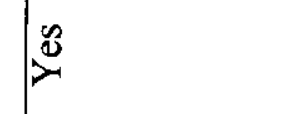 & $\stackrel{\otimes}{\nu}$ & $\overbrace{\nu}^{\infty}$ & 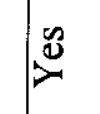 \\
\hline 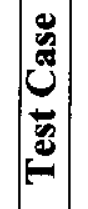 & 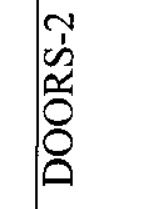 & $\mathbb{z}$ & $\mathbb{Z}$ & $\mathbb{z}$ & 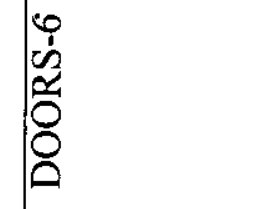 & $\mathbb{z}$ & 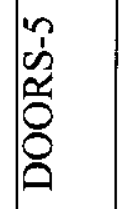 & $\begin{array}{l}n \\
\dot{2} \\
\tilde{2} \\
0 \\
\delta \\
0\end{array}$ & $\begin{array}{l}n \\
\dot{\hat{n}} \\
\tilde{2} \\
\delta \\
0\end{array}$ \\
\hline 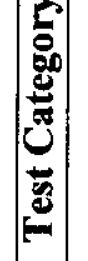 & 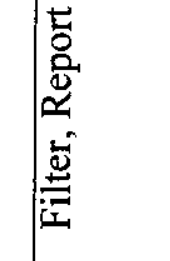 & $\mid \begin{array}{l}\overline{0} \\
\stackrel{0}{0} \\
0 \\
z\end{array}$ & 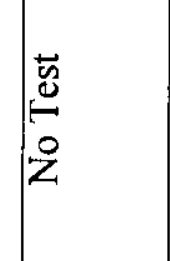 & 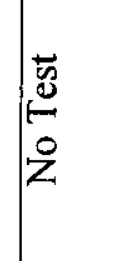 & 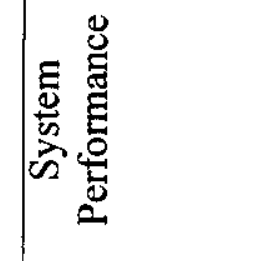 & 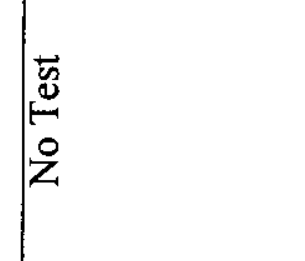 & 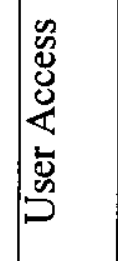 & 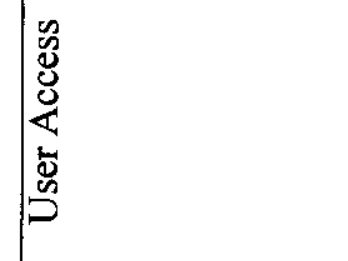 & 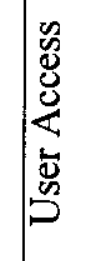 \\
\hline 茑 & 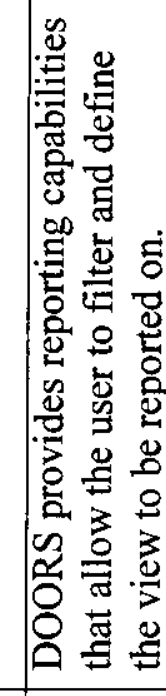 & 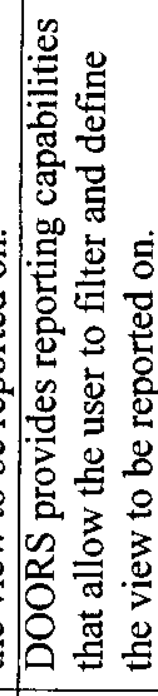 & 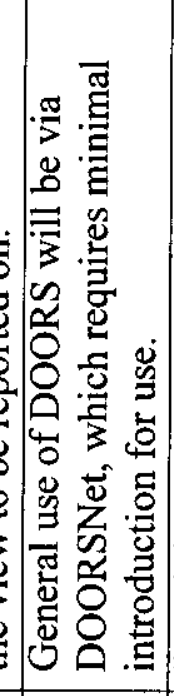 & 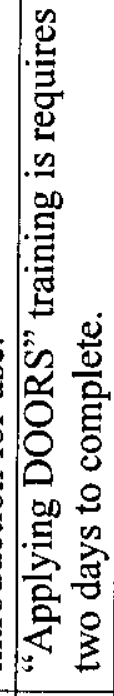 & 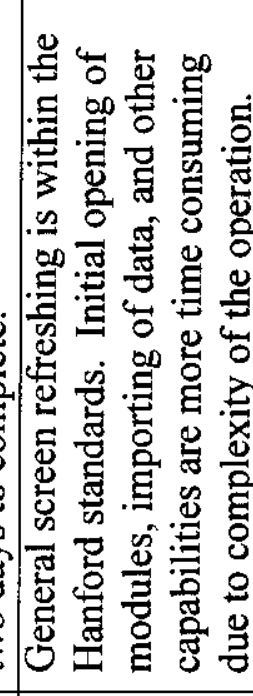 & 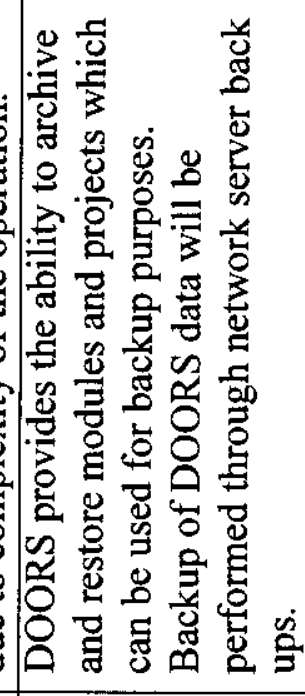 & 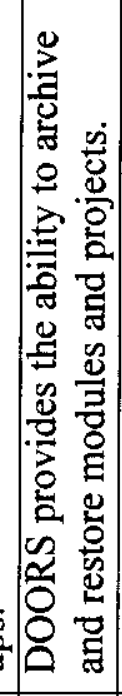 & 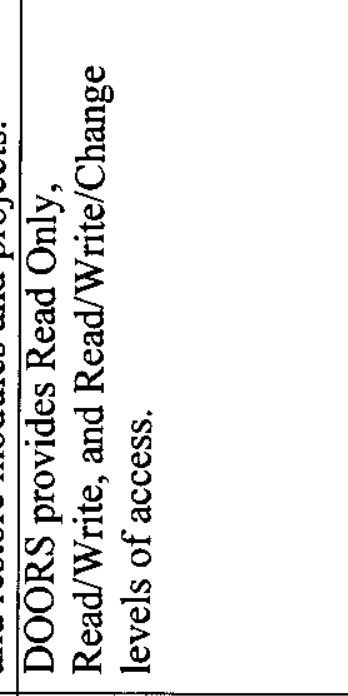 & 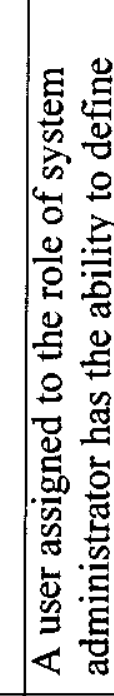 \\
\hline 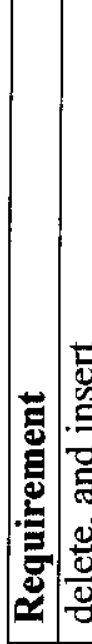 & 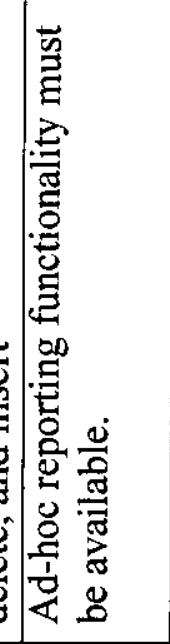 & 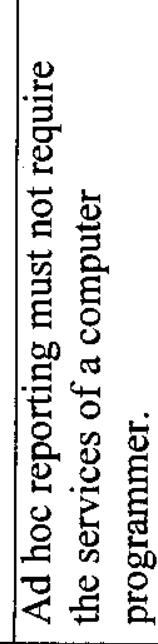 & 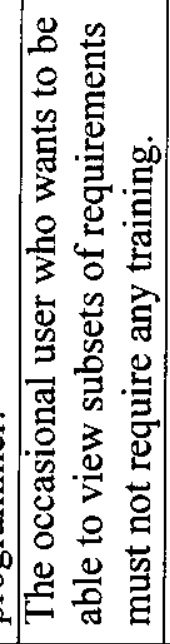 & 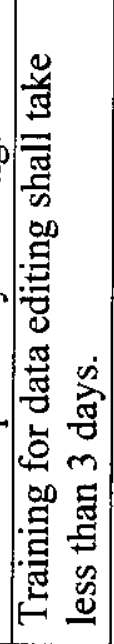 & 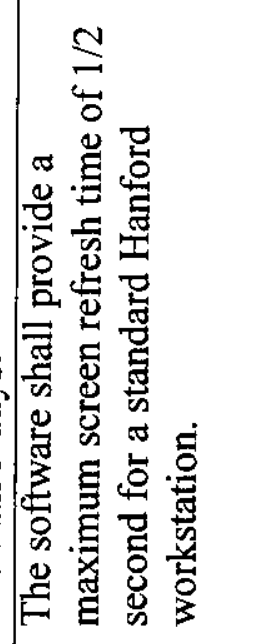 & 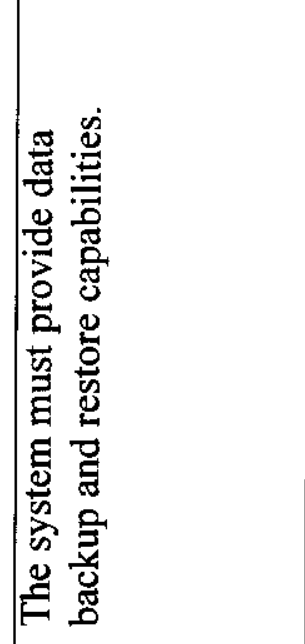 & 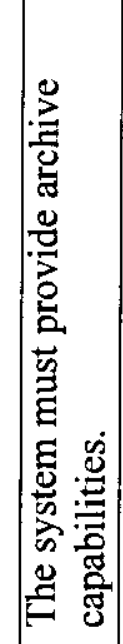 & 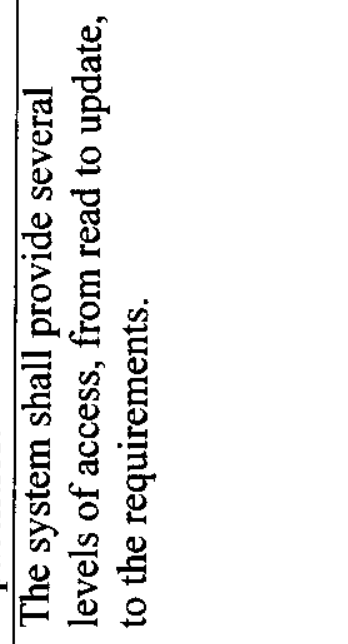 & 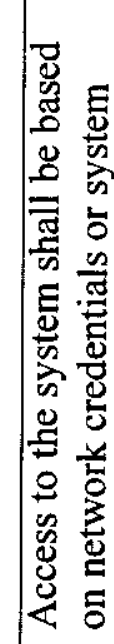 \\
\hline 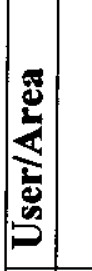 & 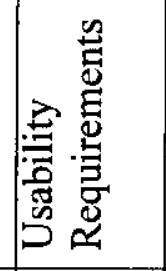 & 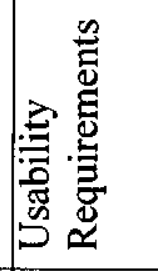 & 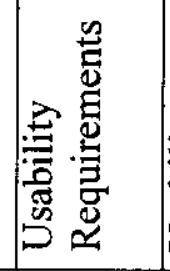 & 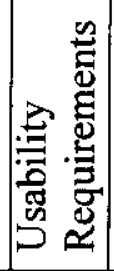 & 总 & 苞 & 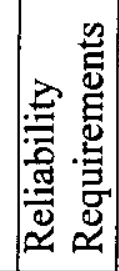 & 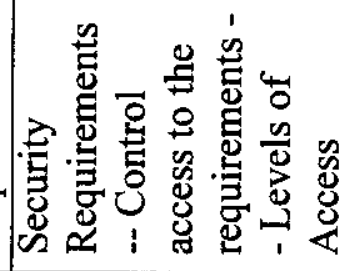 & 空 \\
\hline & $\infty$ & $\infty$ & $\infty$ & హু & $\bar{\sigma}$ & $\sigma$ & אa & 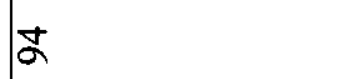 & $\alpha$ \\
\hline
\end{tabular}


RPP-6251 REV. 0

\begin{tabular}{|c|c|c|c|c|c|c|}
\hline$\sum_{\bar{z}}^{\tilde{E}}$ & & 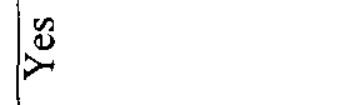 & $\stackrel{\infty}{2}$ & $\stackrel{\infty}{2}$ & $\underbrace{\infty}$ & $\stackrel{\infty}{\infty}$ \\
\hline 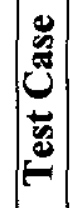 & & $\begin{array}{l}n \\
\tilde{2} \\
\tilde{\tilde{n}} \\
\delta \\
\delta\end{array}$ & $\begin{array}{l}\tilde{n} \\
\hat{n} \\
\tilde{2} \\
0 \\
0\end{array}$ & 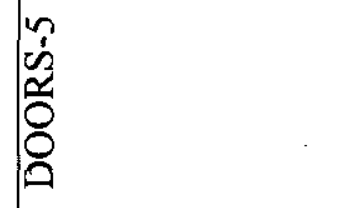 & 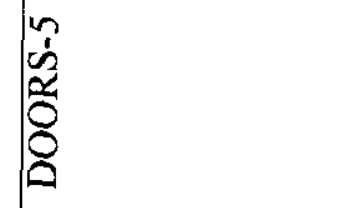 & $\mathbb{z}$ \\
\hline 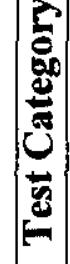 & & 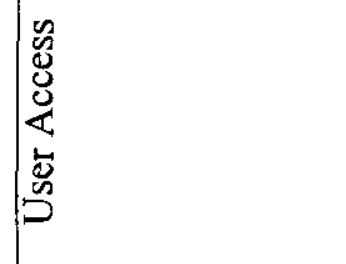 & 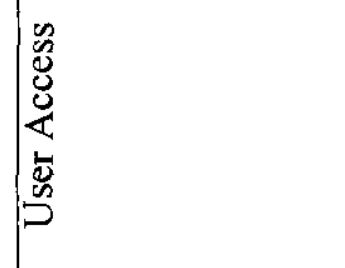 & 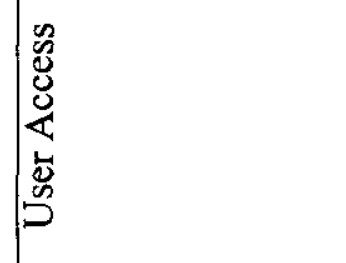 & 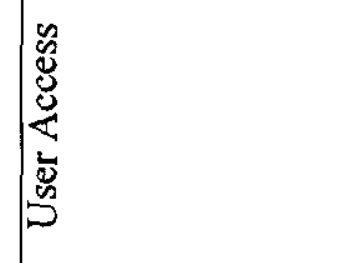 & 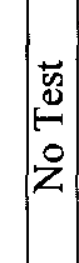 \\
\hline & 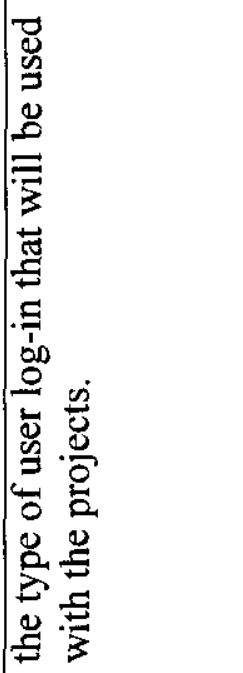 & 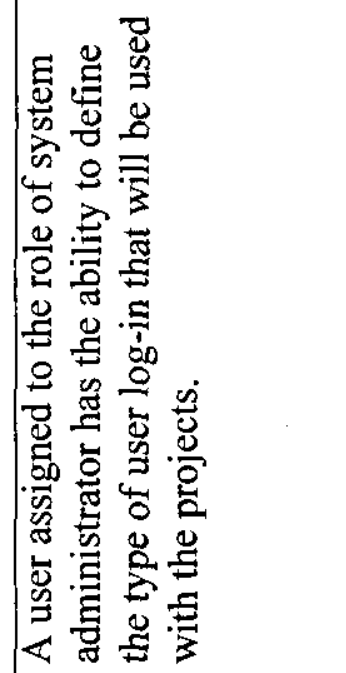 & 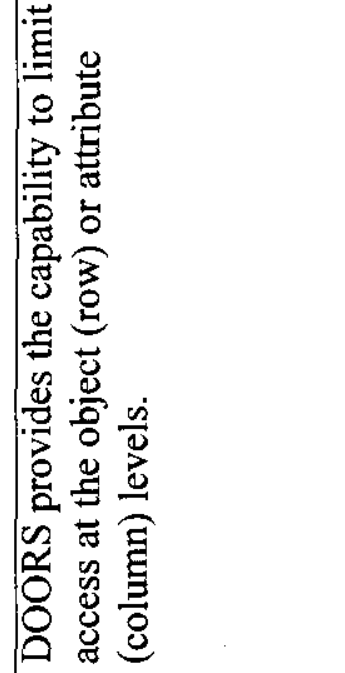 & 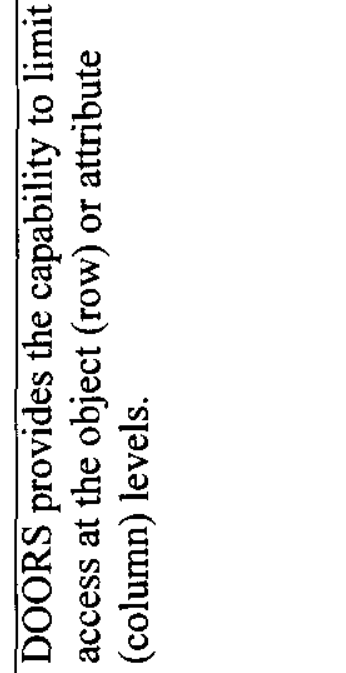 & 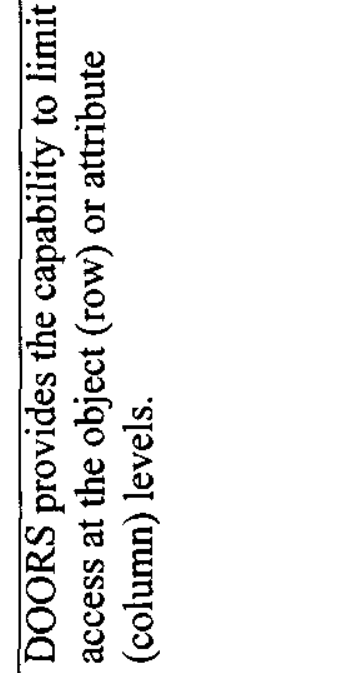 & 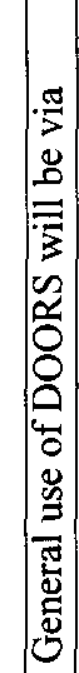 \\
\hline 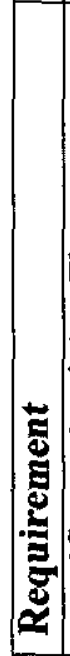 & 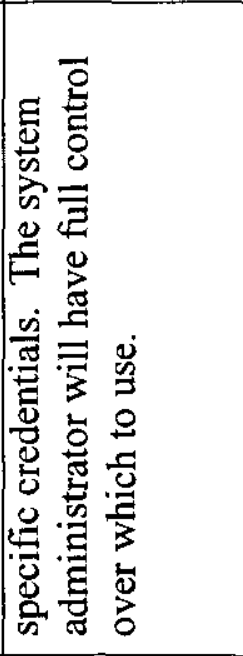 & 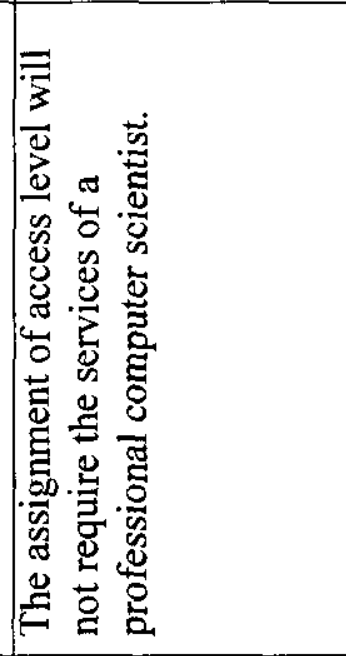 & 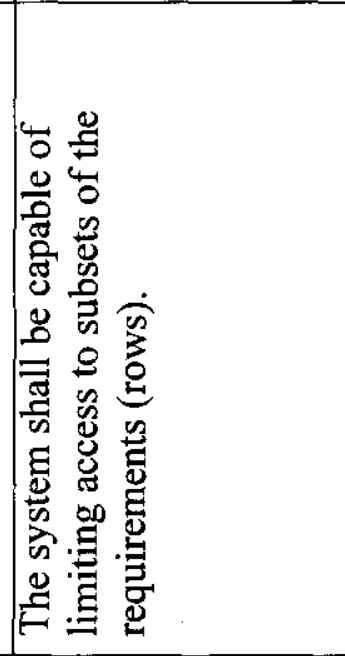 & 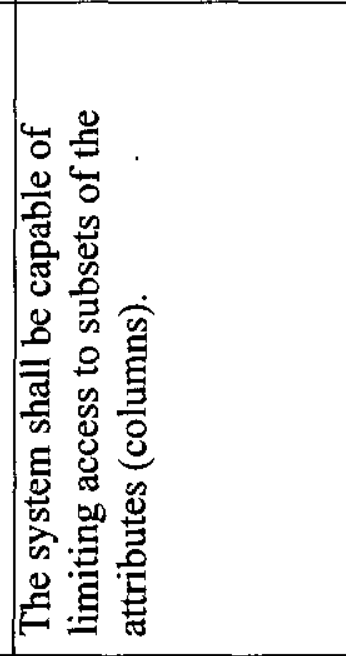 & 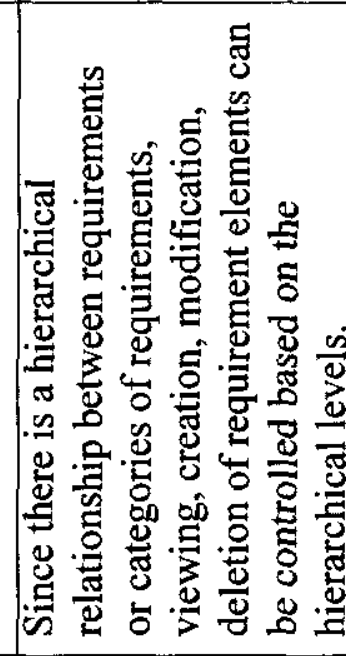 & 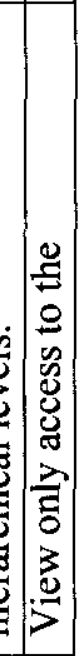 \\
\hline 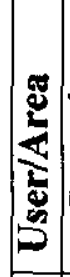 & 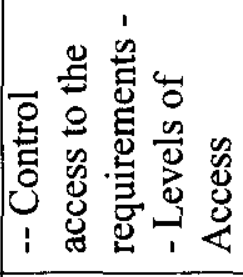 & 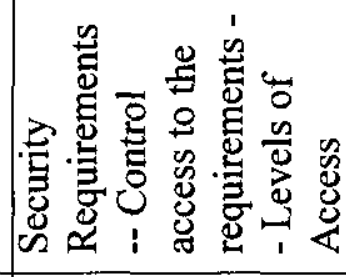 & 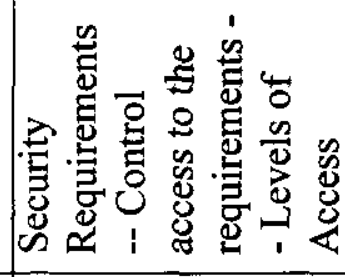 & 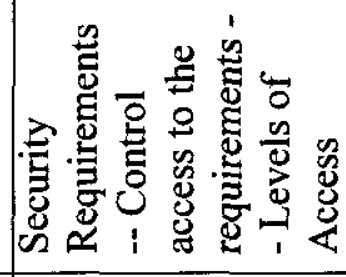 & 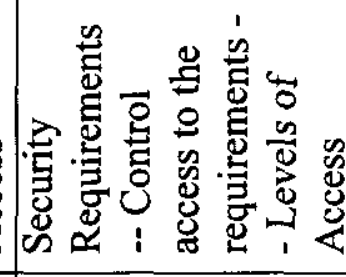 & : \\
\hline 2 & & 12 & î́. & 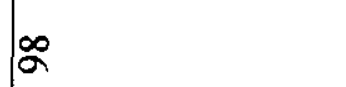 & 2 & $\Xi$ \\
\hline
\end{tabular}


RPP-6251 REV. 0

\begin{tabular}{|c|c|c|c|c|c|}
\hline ثै. & & $D^{\infty}$ & $y^{y}$ & $\stackrel{\infty}{2}$ & $\stackrel{\infty}{\varnothing}$ \\
\hline 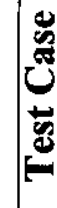 & & $\varangle$ & $\mathbb{z}$ & 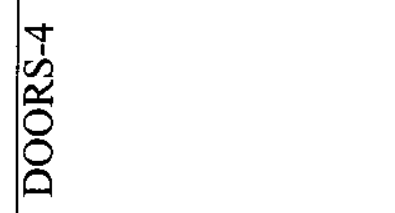 & 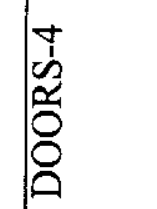 \\
\hline 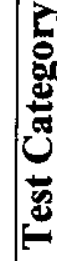 & & 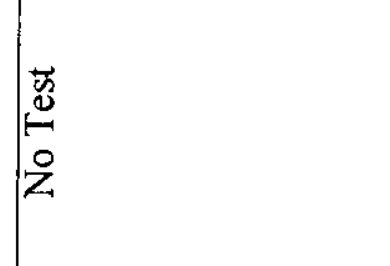 & 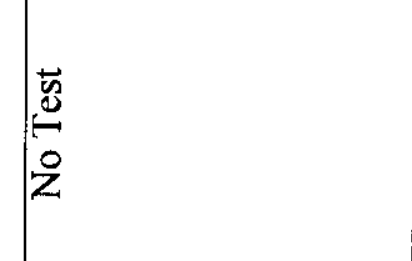 & 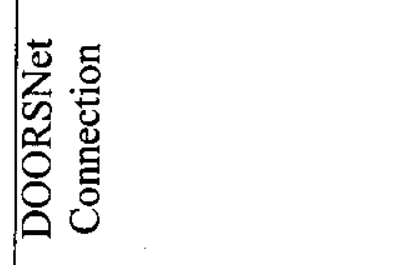 & 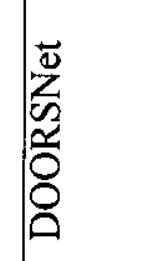 \\
\hline छ & 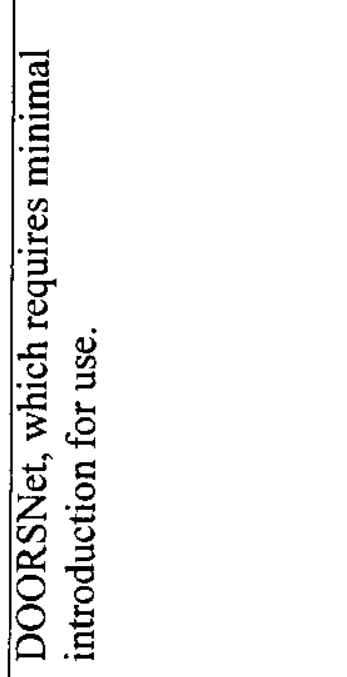 & 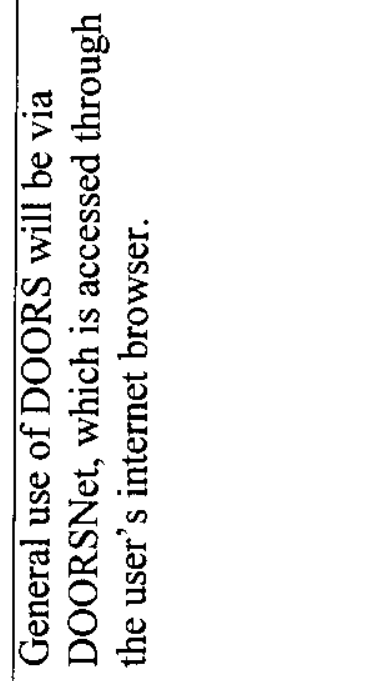 & 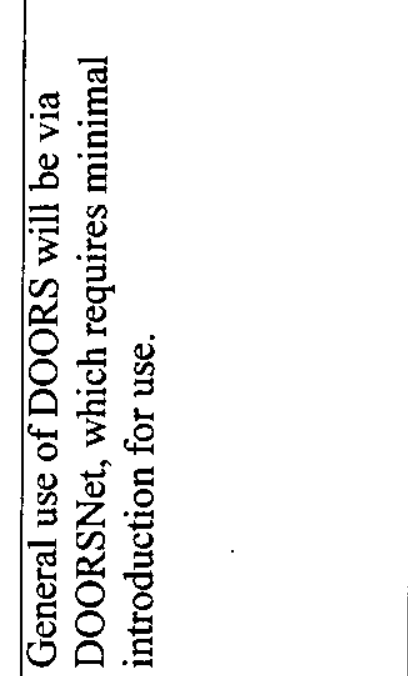 & 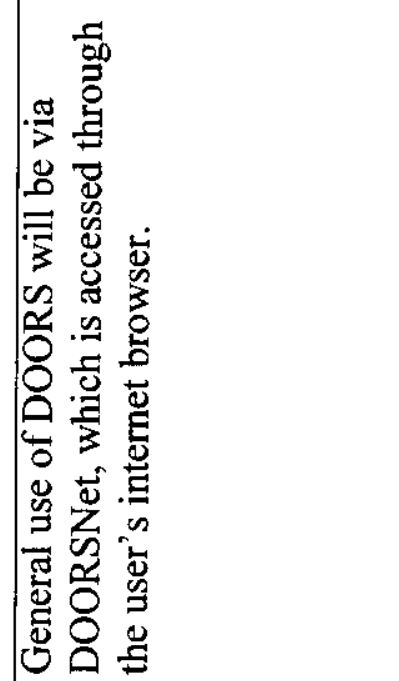 & 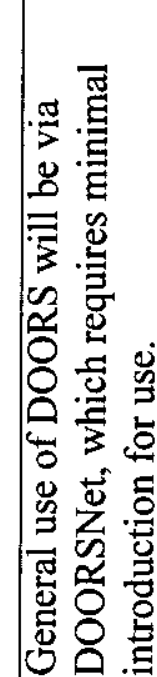 \\
\hline & 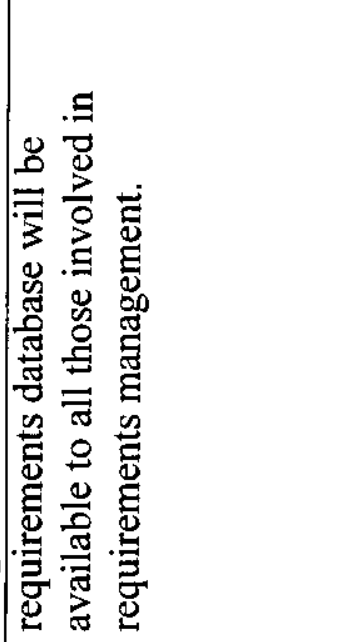 & 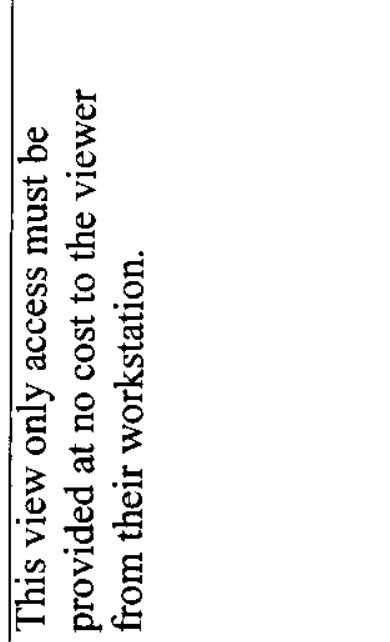 & 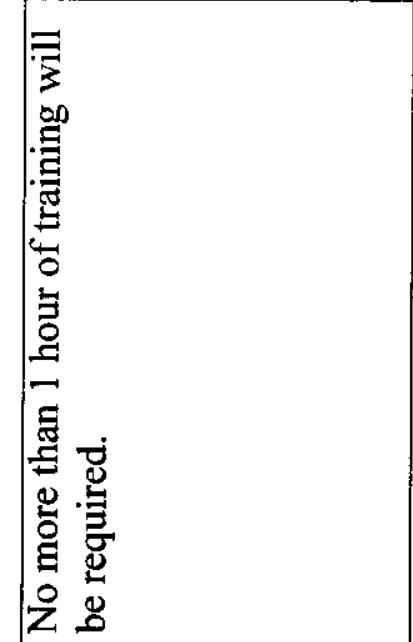 & 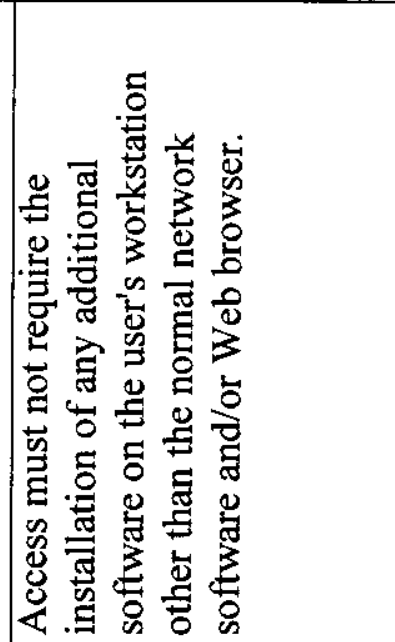 & 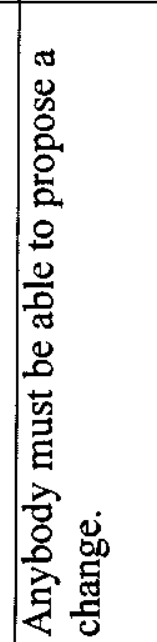 \\
\hline & 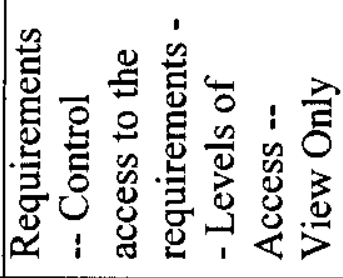 & 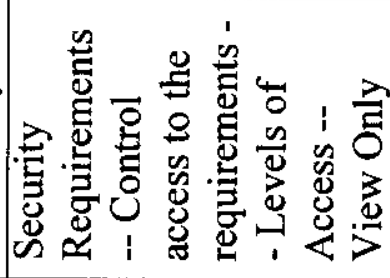 & 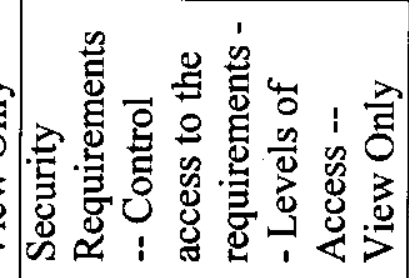 & 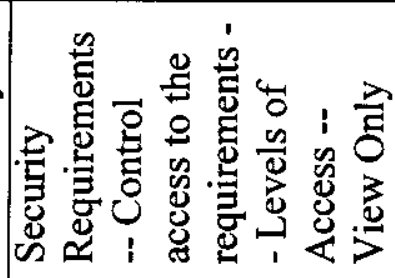 & 总 \\
\hline$z$ & & 흐 & SO & $\hat{\varrho}$ & $\underset{\Xi}{\mathbb{O}}$ \\
\hline
\end{tabular}


RPP-6251 REV. 0

\begin{tabular}{|c|c|c|c|c|c|}
\hline $\mid$ & & $\frac{n}{2}$ & $\stackrel{0}{2}$ & $\stackrel{8}{2}$ & $\ddot{\infty}$ \\
\hline 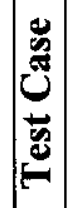 & & $\mathbb{Z}$ & 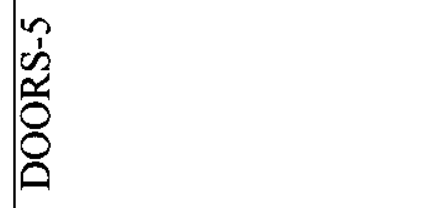 & 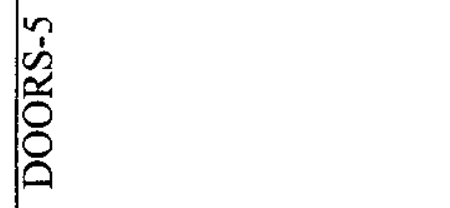 & 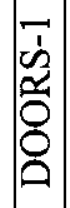 \\
\hline 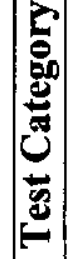 & & 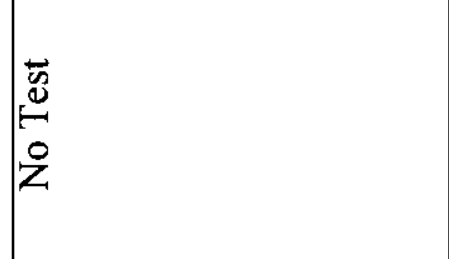 & 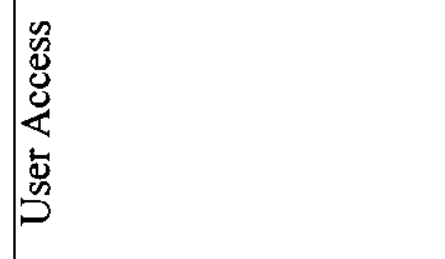 & 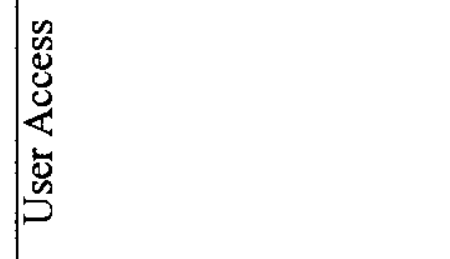 & $\underline{\underline{y}}$ \\
\hline 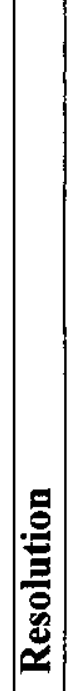 & & 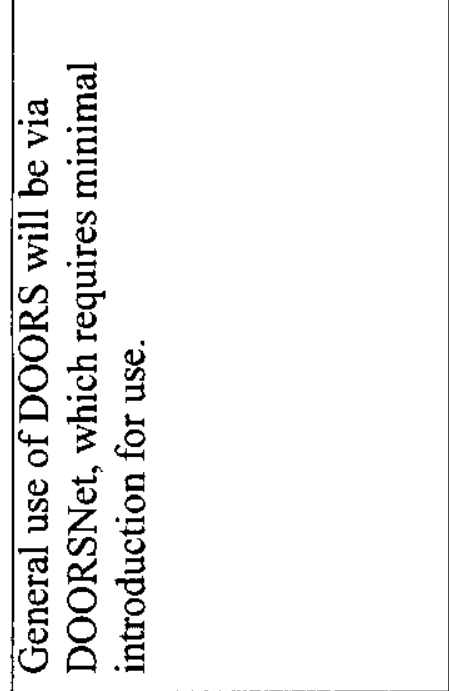 & 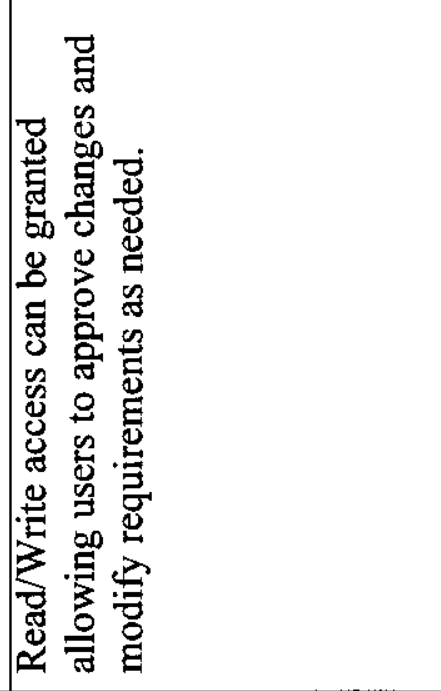 & 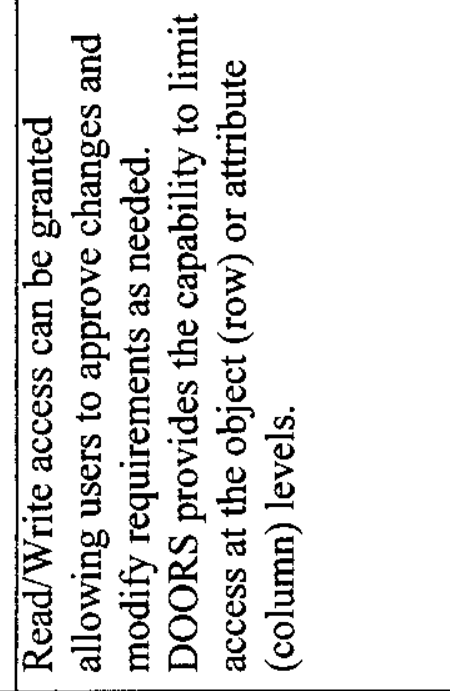 & 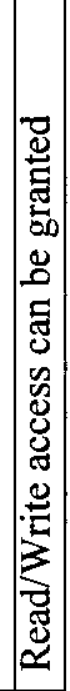 \\
\hline 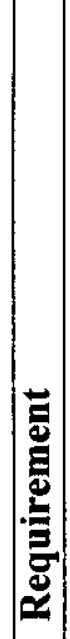 & & 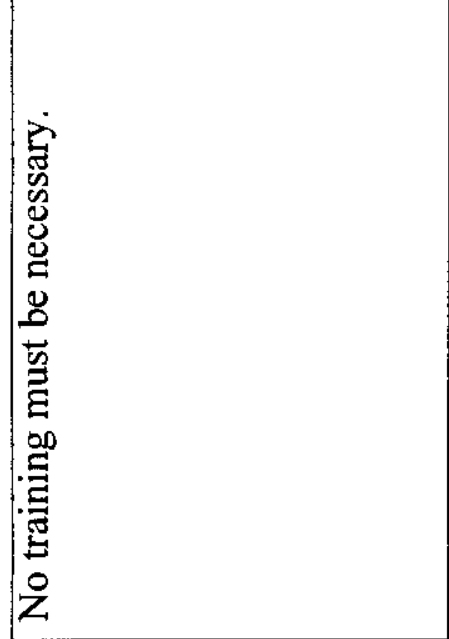 & 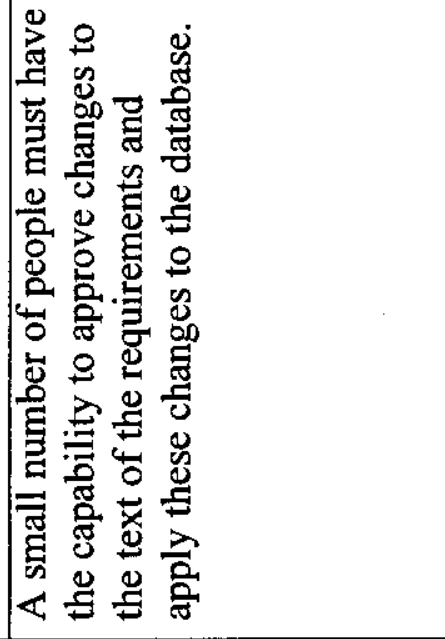 & 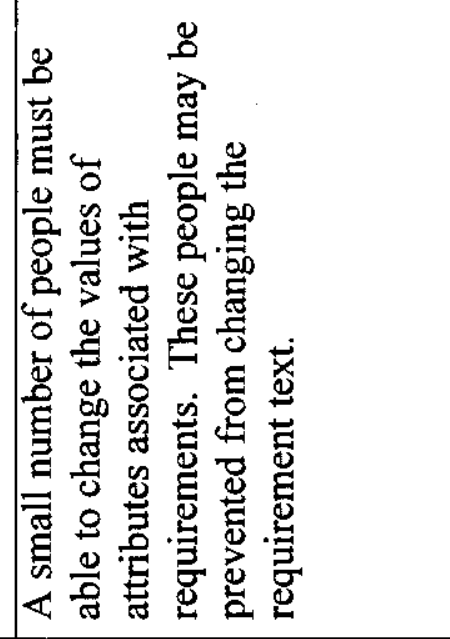 & 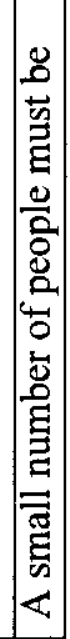 \\
\hline 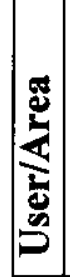 & 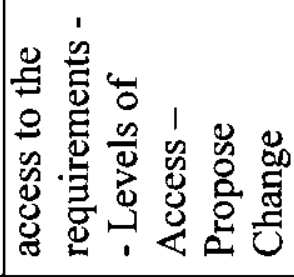 & 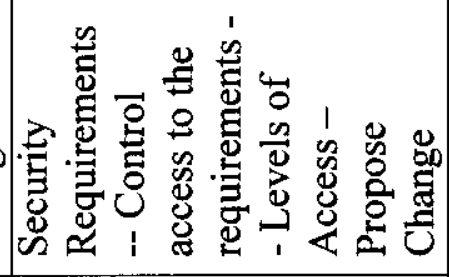 & 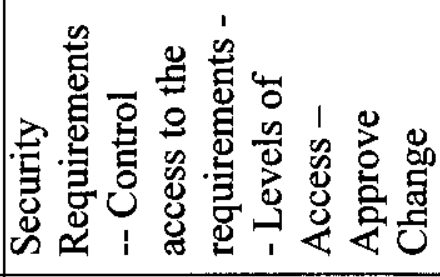 & 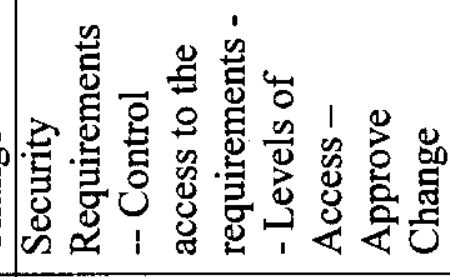 & 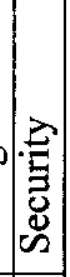 \\
\hline 2 & & $\cong$ & 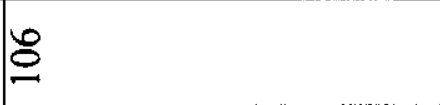 & $\hat{\varrho}$ & $\stackrel{\infty}{0}$ \\
\hline
\end{tabular}


RPP-6251 REV. 0

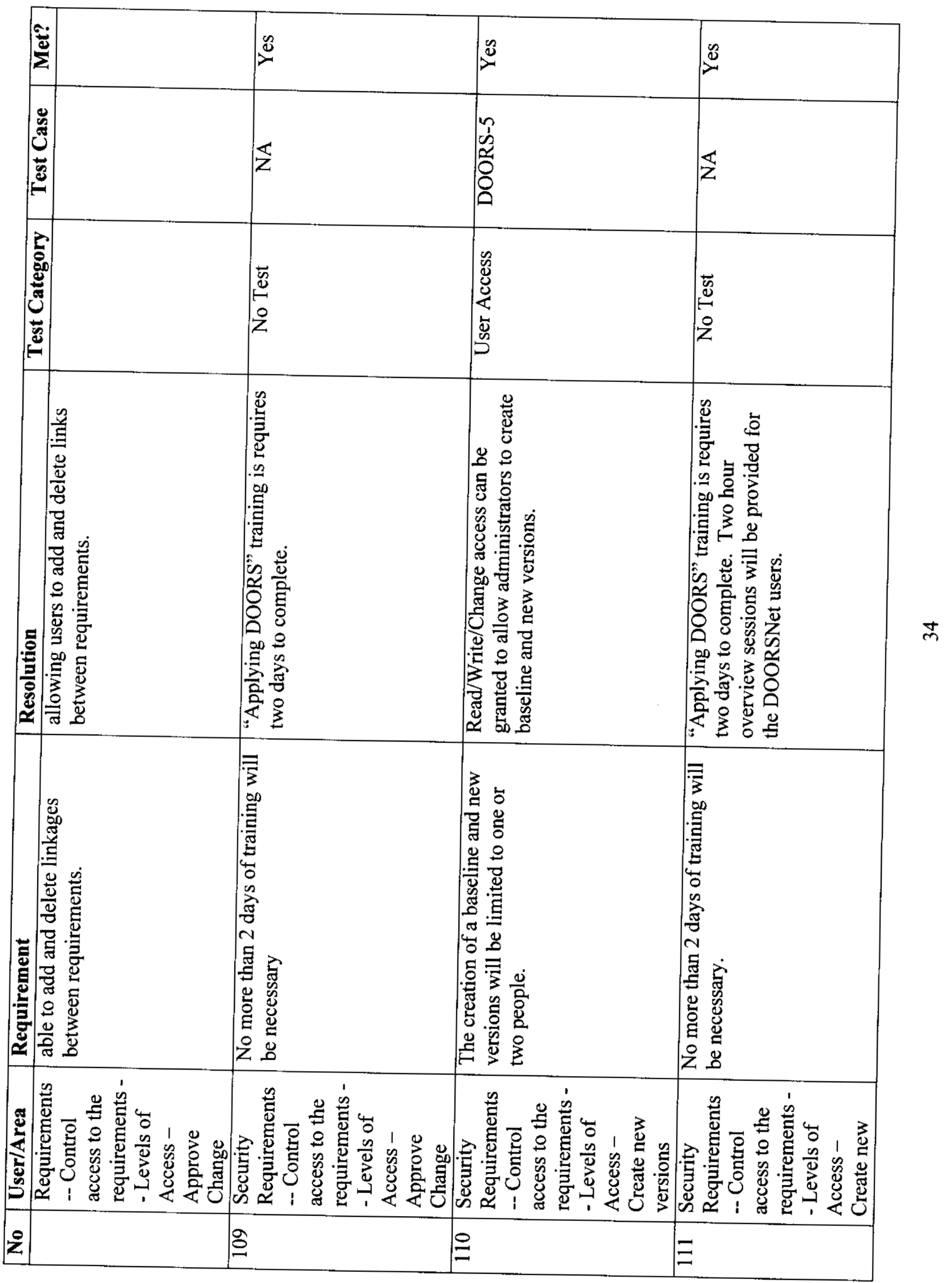


RPP-6251 REV. 0

\begin{tabular}{|c|c|c|c|c|c|c|c|c|c|c|c|}
\hline & $x^{2}$ & $\stackrel{\infty}{\rightleftharpoons}$ & $\underset{\nu}{*}$ & $\underset{\nu}{\infty}$ & $\mathscr{\infty}_{\nu}$ & $\stackrel{\infty}{\infty}$ & $\underset{z}{\infty}$ & $\overbrace{}^{\infty}$ & $\stackrel{0}{\nu}$ & $\nu^{\infty}$ & $\nu^{\infty}$ \\
\hline & $\mathbb{Z}$ & $\mathbb{z}$ & 层 & $\overleftrightarrow{z}$ & 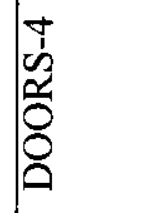 & 文 & $\mathbb{z}$ & $\ll$ & $\mathbb{z}$ & $\begin{array}{l}0 \\
0 \\
0 \\
0 \\
0 \\
0 \\
0 \\
0\end{array}$ & 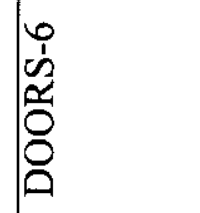 \\
\hline & $\begin{array}{l}\tilde{y} \\
\stackrel{y}{0} \\
0 \\
z\end{array}$ & 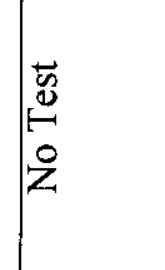 & $\mid \begin{array}{l}\vec{n} \\
\stackrel{0}{0} \\
0 \\
z\end{array}$ & $\begin{array}{l}\tilde{3} \\
\stackrel{3}{8} \\
0 \\
z\end{array}$ & 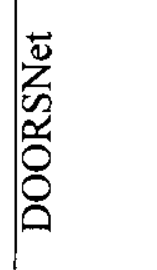 & 苟 & 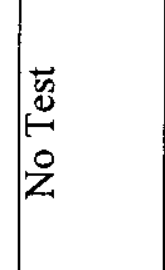 & $\begin{array}{l}\text { 荀 } \\
\frac{0}{0} \\
0 \\
z\end{array}$ & 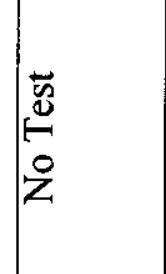 & 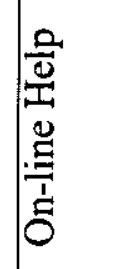 & 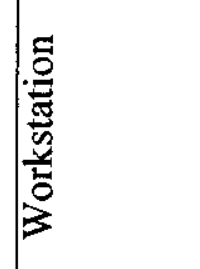 \\
\hline & 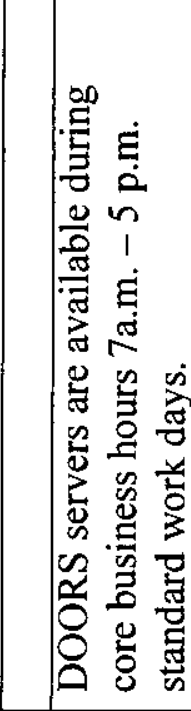 & 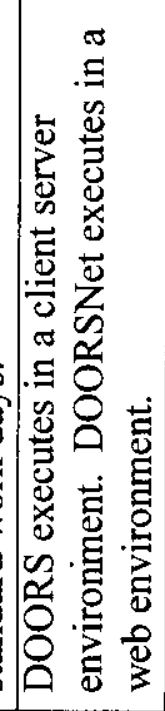 & 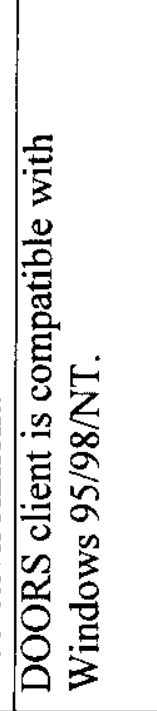 & 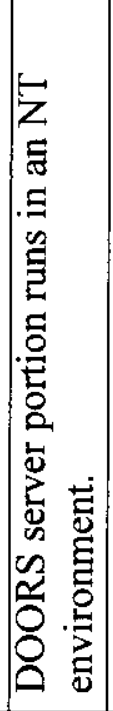 & 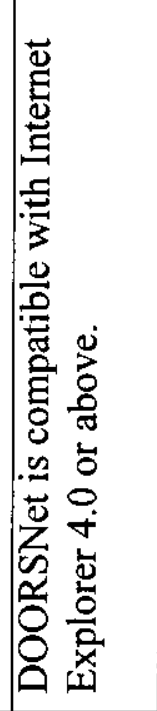 & 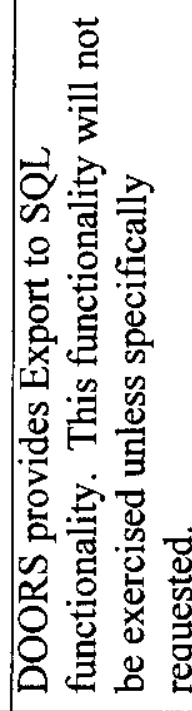 & 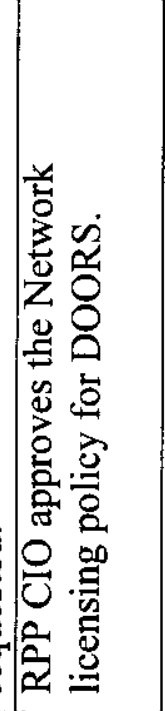 & 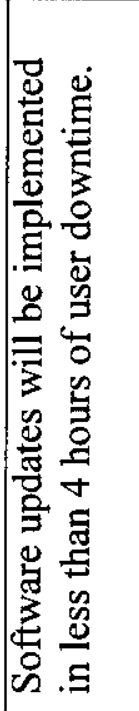 & 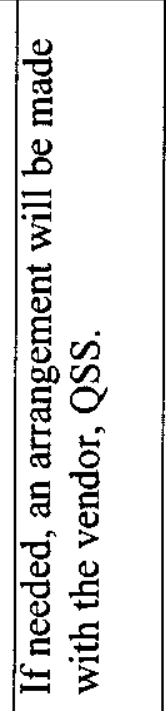 & 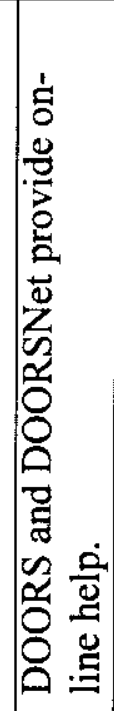 & 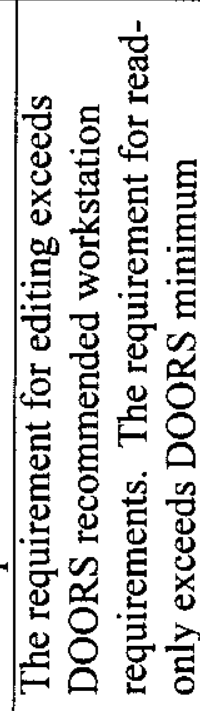 \\
\hline 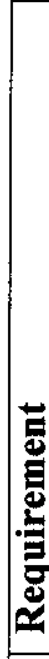 & 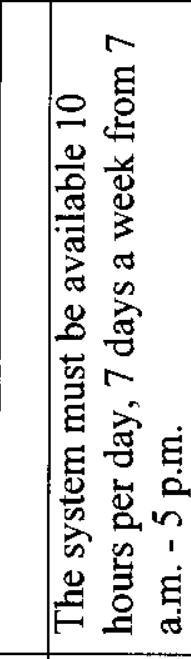 & 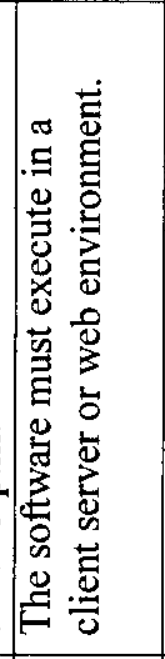 & 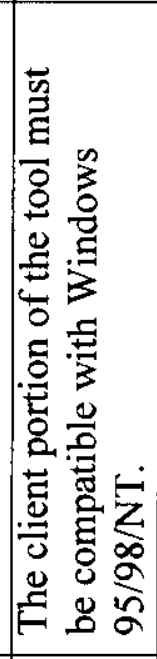 & 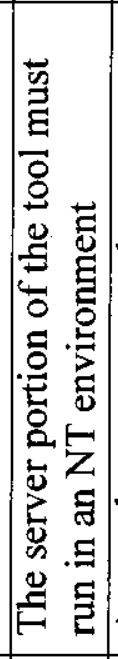 & 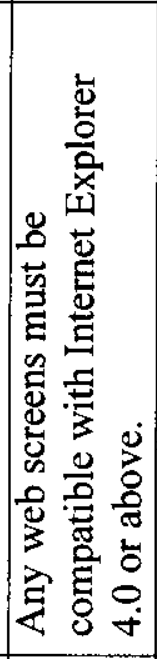 & 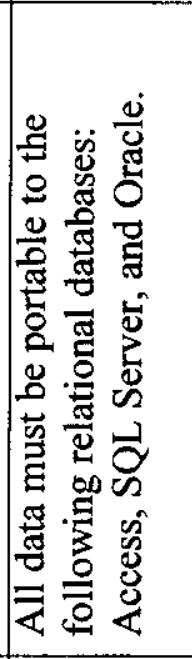 & 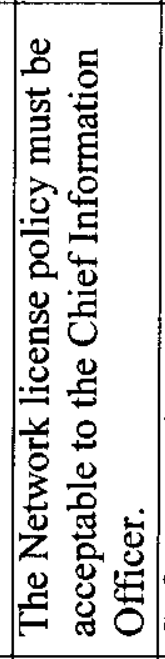 & 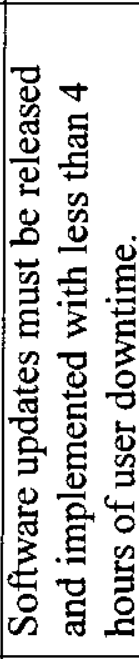 & 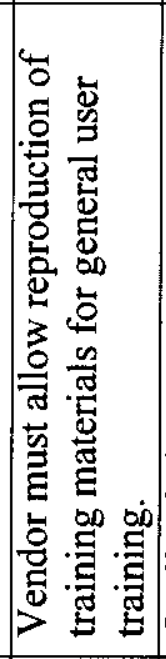 & 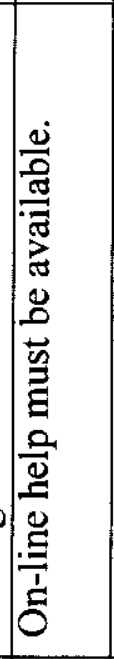 & 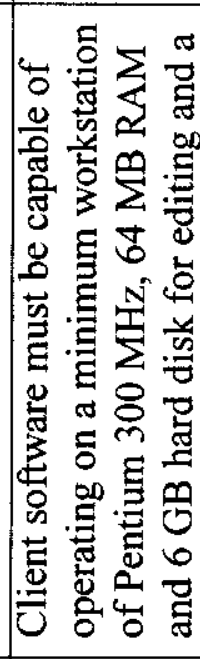 \\
\hline & 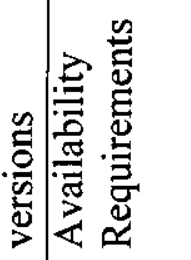 & 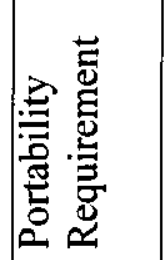 & 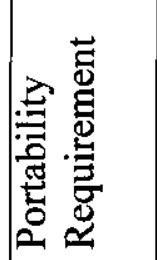 & 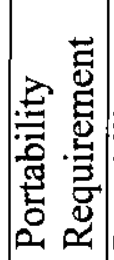 & 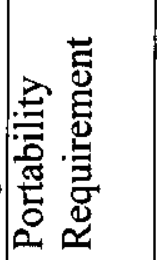 & 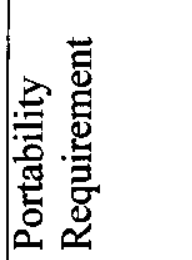 & 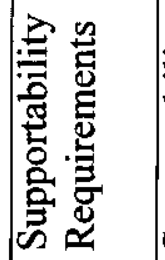 & 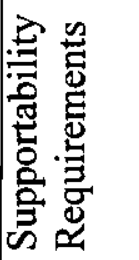 & 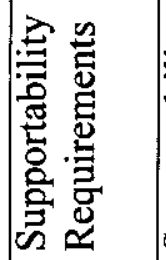 & 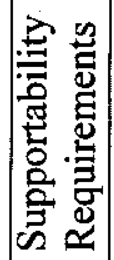 & 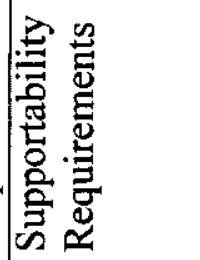 \\
\hline & $\cong$ & $\cong$ & $\Xi$ & 1- & 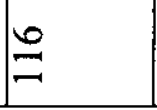 & - & & $\stackrel{\partial}{=}$ & & $\approx$ & -2 \\
\hline
\end{tabular}


RPP-6251 REV. 0

\begin{tabular}{|c|c|c|c|c|c|}
\hline$\sum^{5}$ & & $\stackrel{\infty}{\infty}$ & $\infty$ & $\stackrel{\infty}{2}$ & $\stackrel{\nu}{\nu}$ \\
\hline 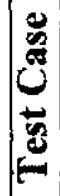 & & 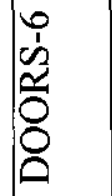 & $\mathbb{Z}$ & $\overleftrightarrow{Z}$ & 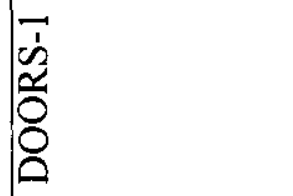 \\
\hline 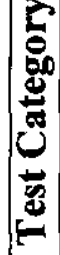 & & 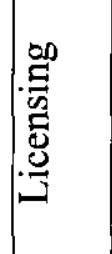 & 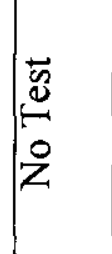 & 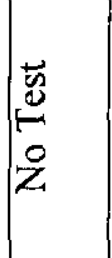 & 㒸 \\
\hline 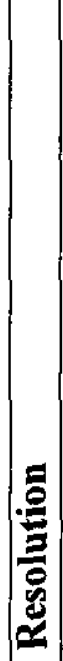 & 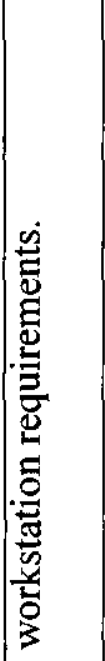 & 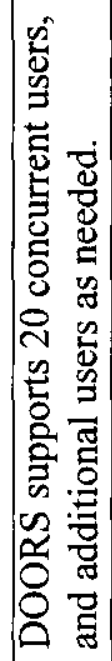 & 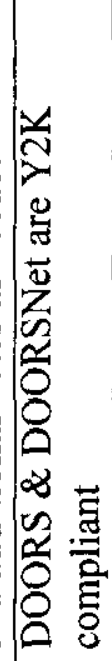 & 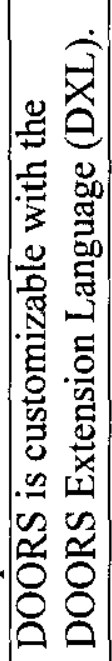 & 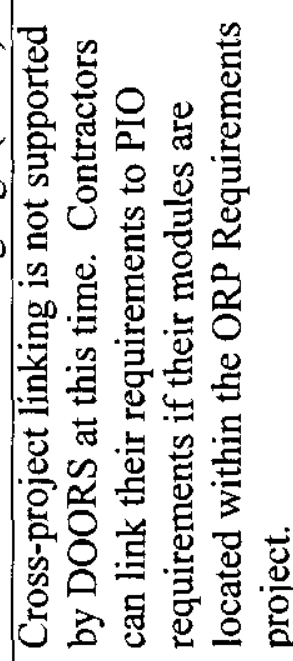 \\
\hline 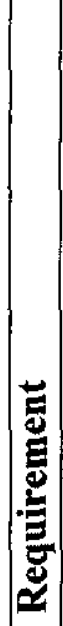 & 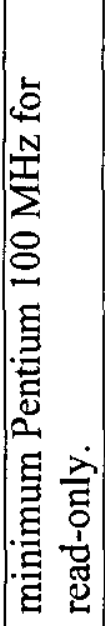 & 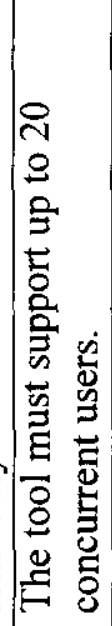 & 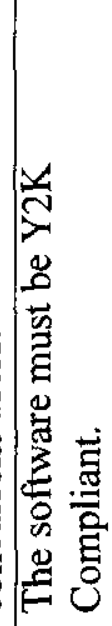 & 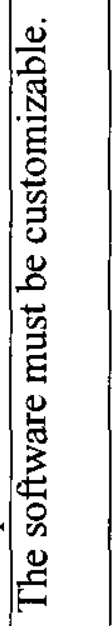 & 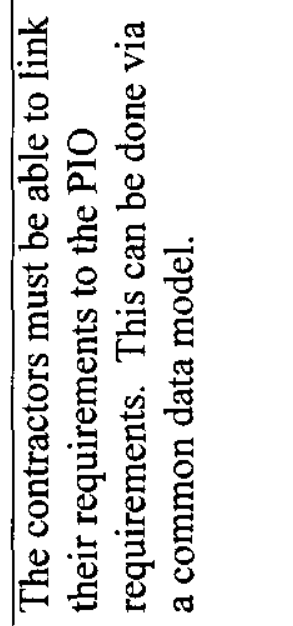 \\
\hline 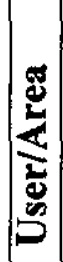 & & 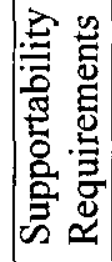 & 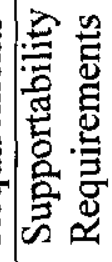 & 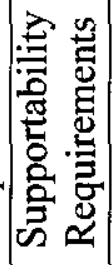 & 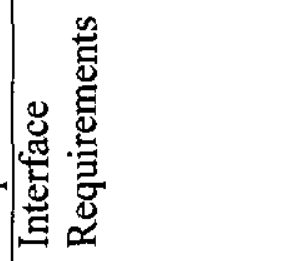 \\
\hline $\mathbf{Z}$ & & 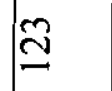 & $\stackrel{+}{\sim}$ & $\cong$ & $\stackrel{\sim}{\stackrel{2}{2}}$ \\
\hline
\end{tabular}


RPP-6251 REV. 0

\section{APPENDIX B - TEST CASES AND RESULTS}

Completed test cases with results will be inserted into the test plan, at this point, after all tests have been performed. The following test cases will be performed.

\begin{tabular}{|l|l|}
\hline \multicolumn{1}{|c|}{ Test Case Number } & \multicolumn{1}{|c|}{ Test Case Subject Area } \\
\hline DOORS-1 & DOORS General Use (Create Project, Module, Attribute, Link, Import) \\
\hline DOORS-2 & DOORS General Use (Views, Filters, Sort, Reporting, Export, Baseline, History) \\
\hline DOORS-3 & DOORS Change Proposal System \\
\hline DOORS-4 & DOORSNet General Use \\
\hline DOORS-5 & DOORS User Access \\
\hline DOORS-6 & DOORS and DOORSNet System Testing (Broken down into sub-tests) \\
& 6.01 - DOORS PC Client General System Test \\
& $6.02-$ DOORSNet Client General System Test \\
& 6.03 - DOORS PC Manual Single User Performance Test \\
& $6.04-$ DOORSNet Manual Single User Performance Test \\
& 6.05 - DOORS PC Manual Multiple Users Performance Test \\
& 6.06 - DOORSNet Manual Multiple Users Performance Test \\
\hline
\end{tabular}




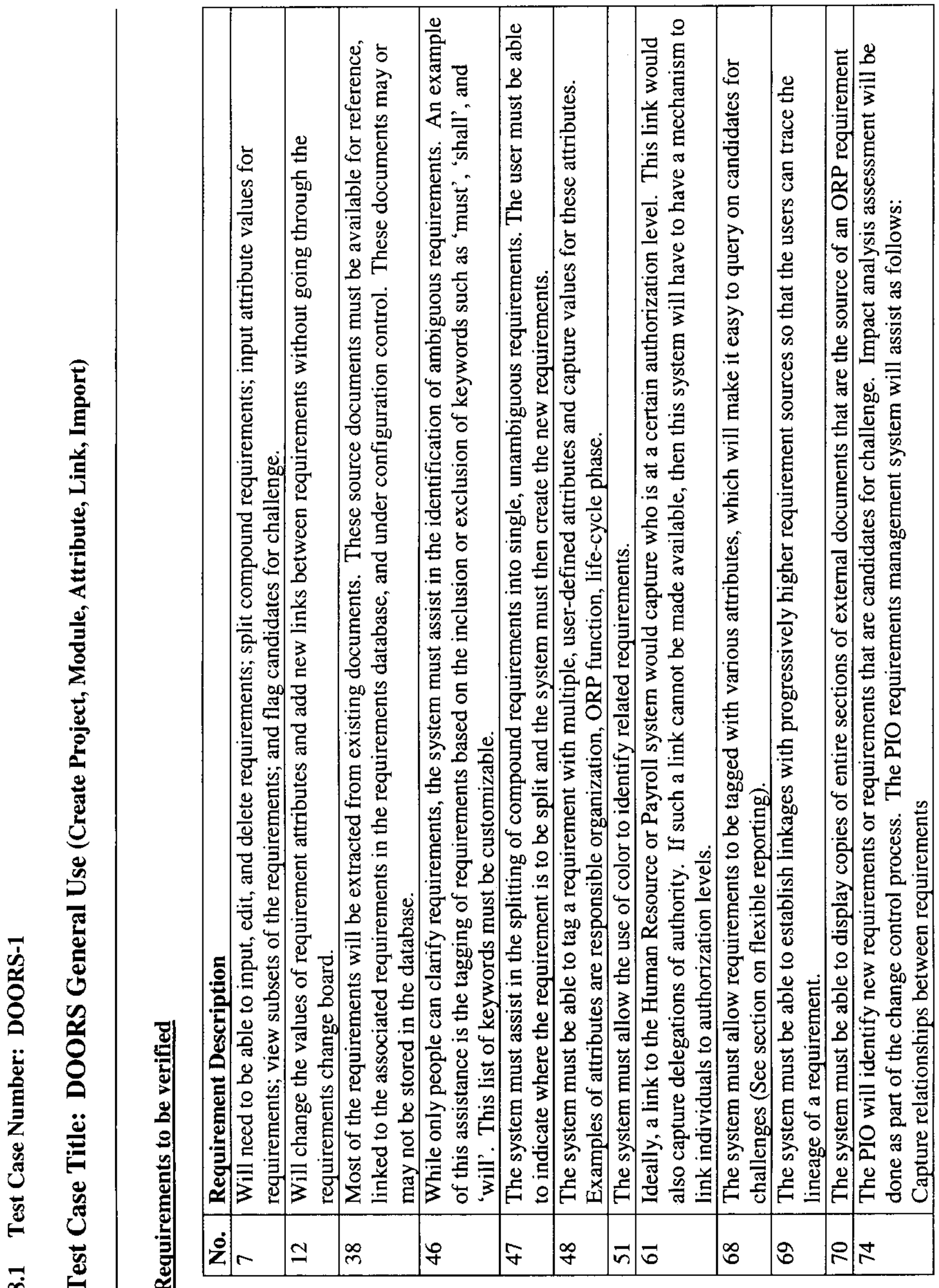



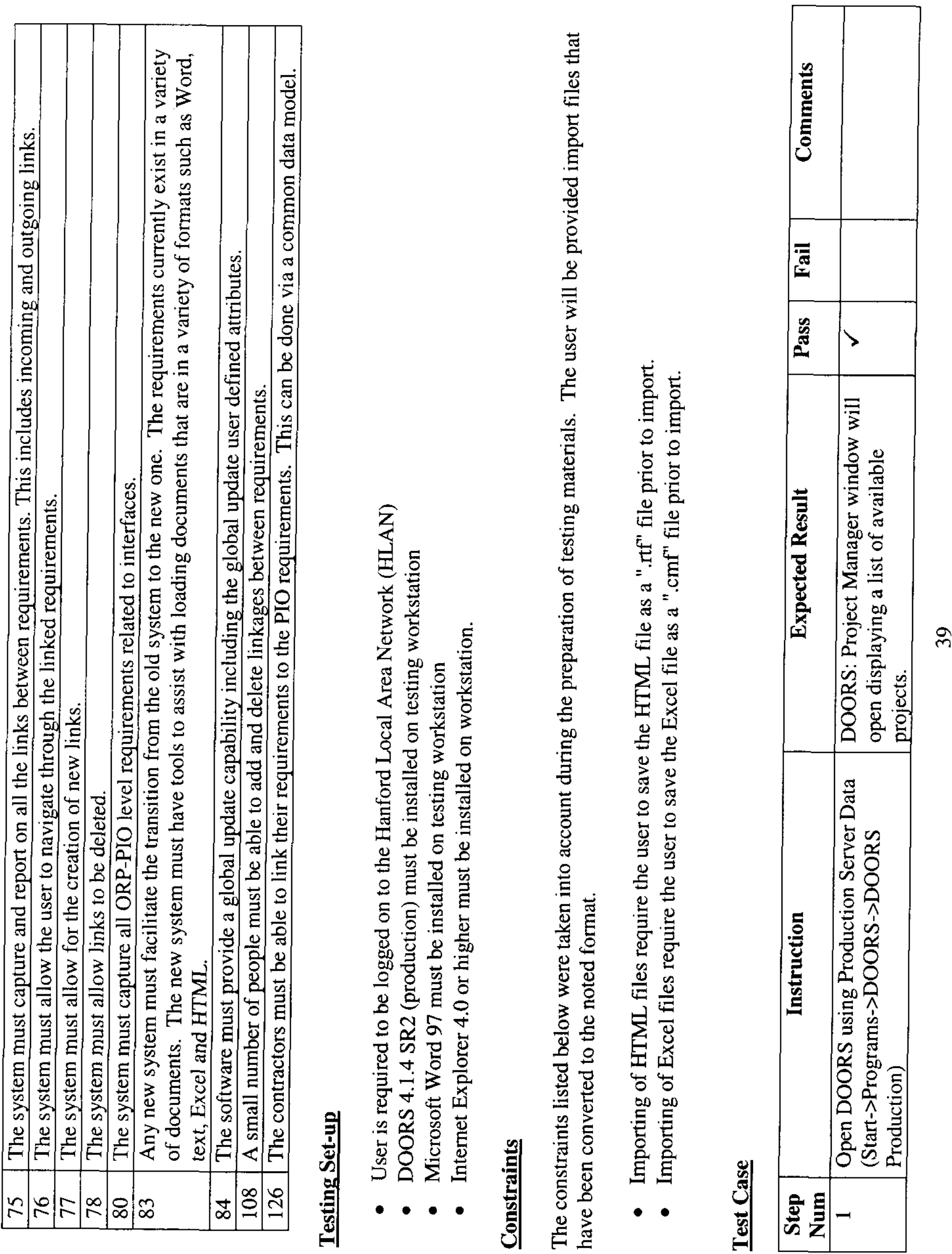


\section{RPP-6251 REV. 0}

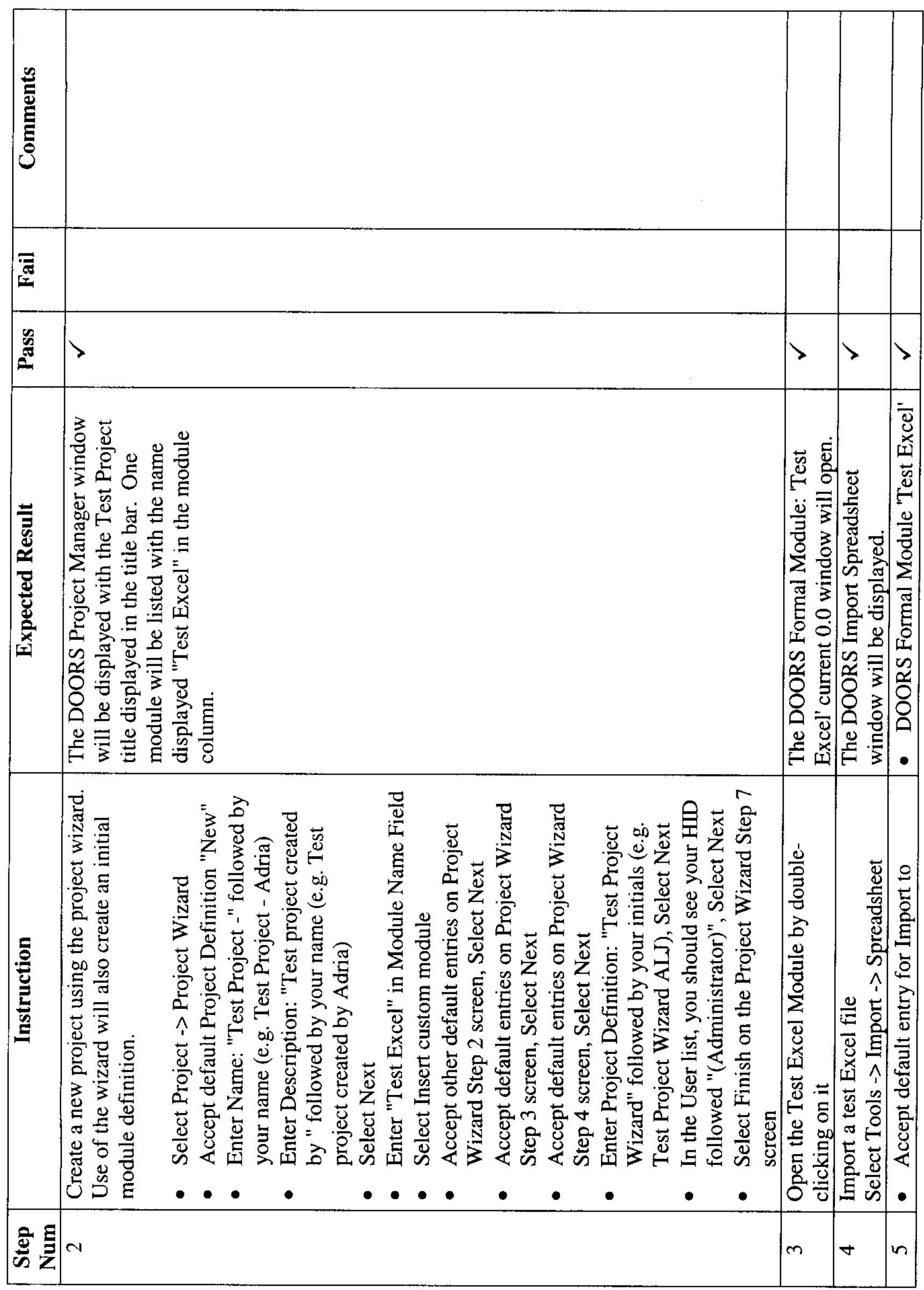




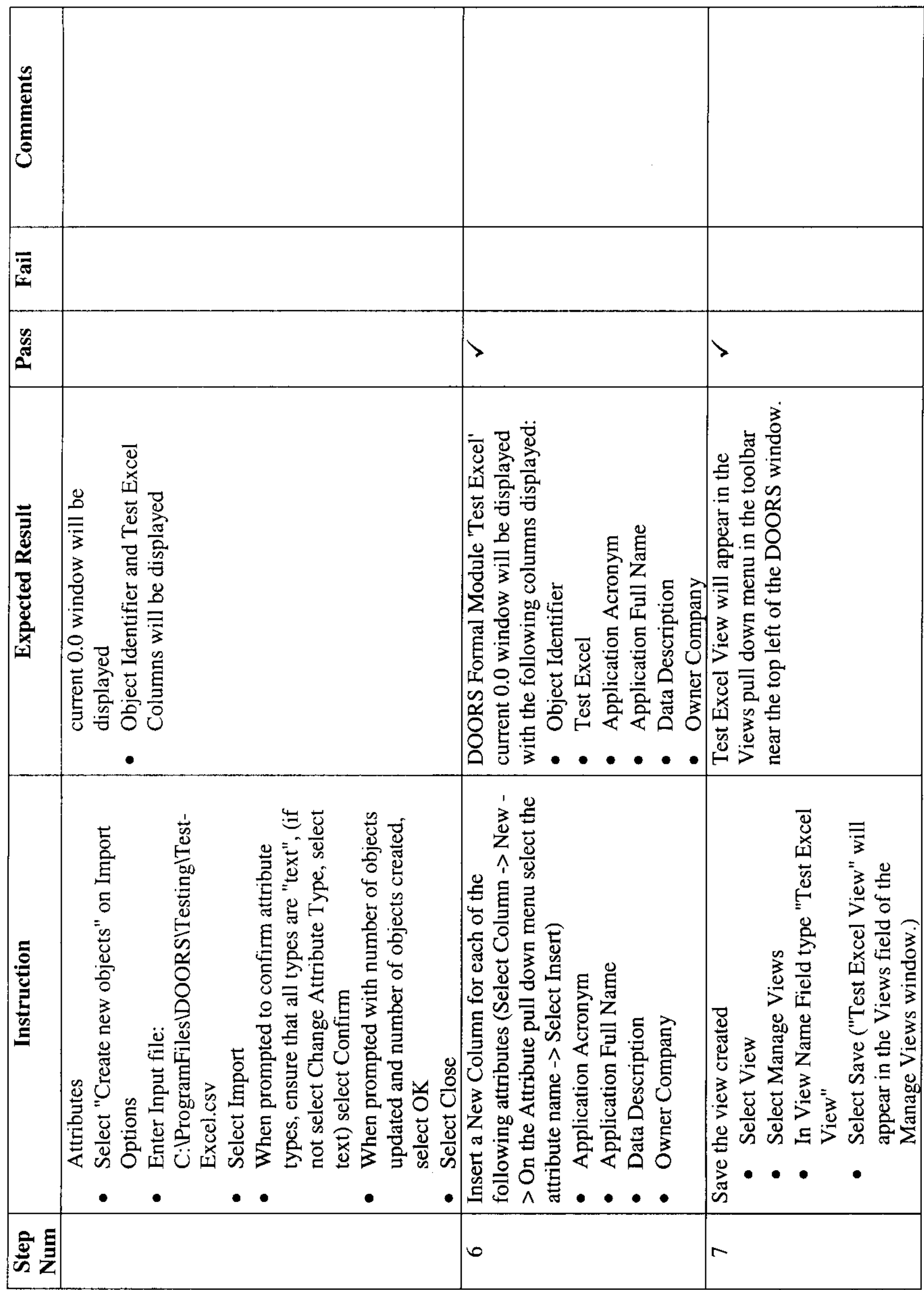


RPP-6251 REV. 0

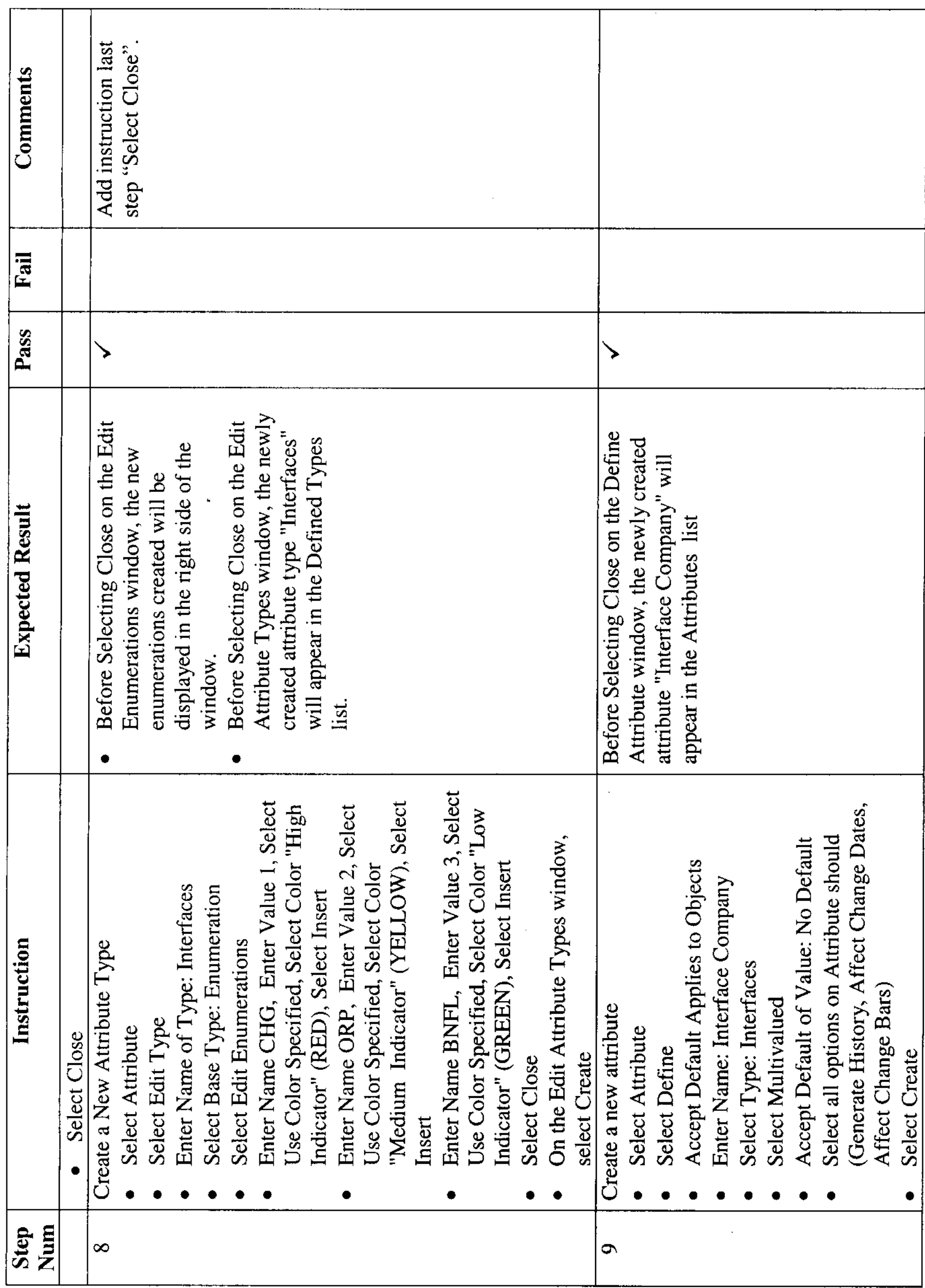


RPP-6251 REV. 0

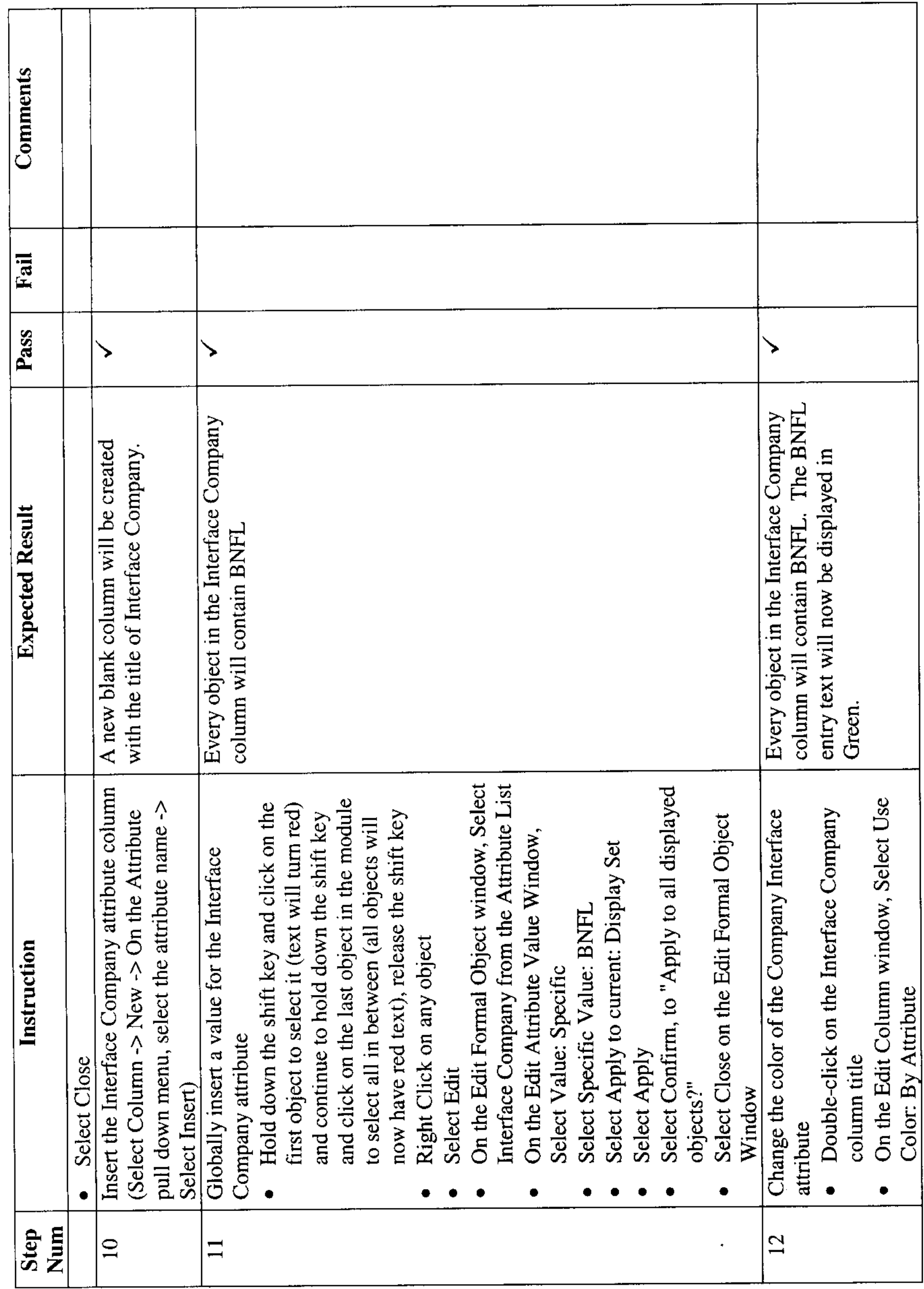


RPP-6251 REV. 0

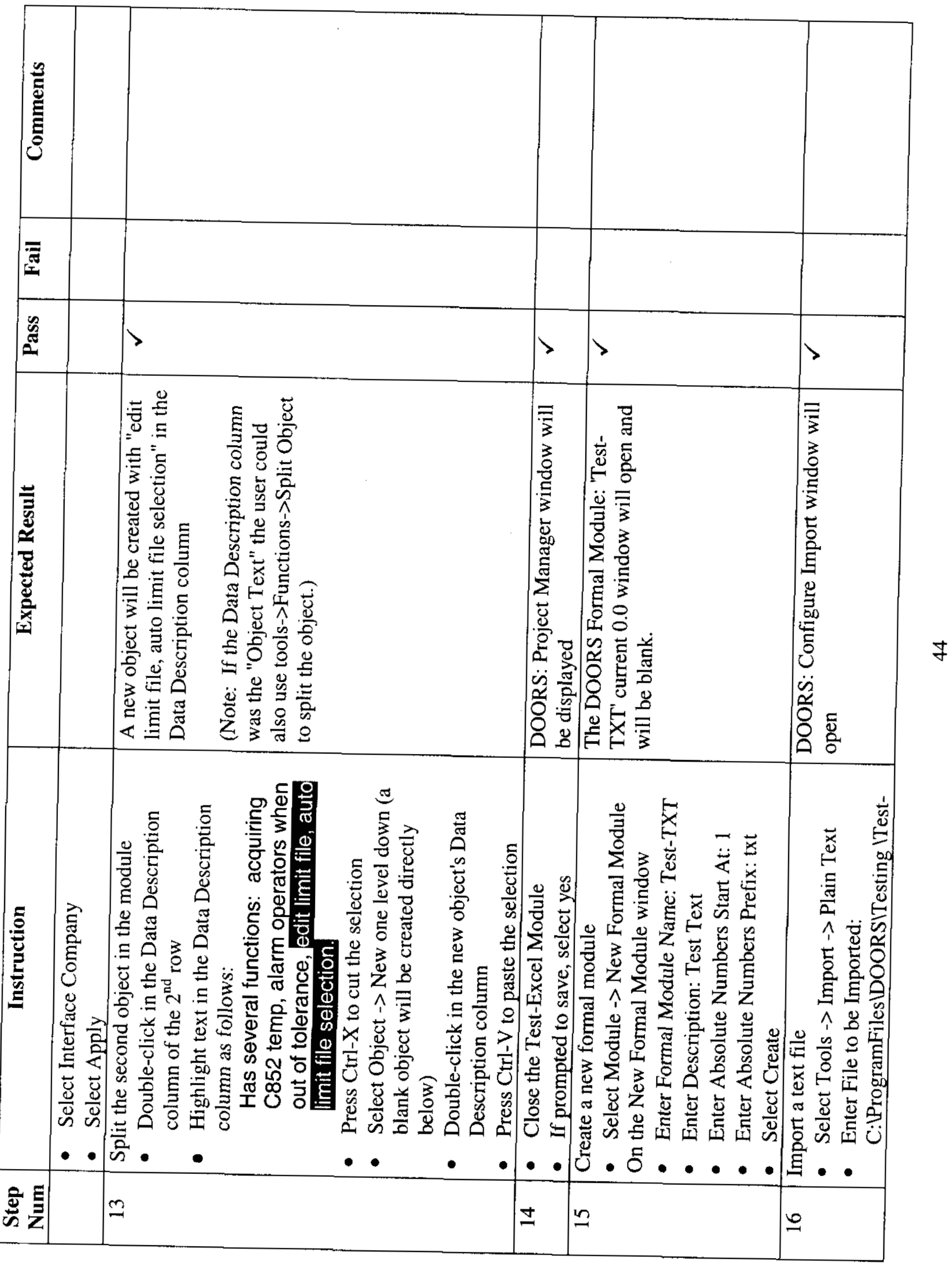


RPP-6251 REV. 0

\begin{tabular}{|c|c|c|c|c|c|c|}
\hline & & & & & 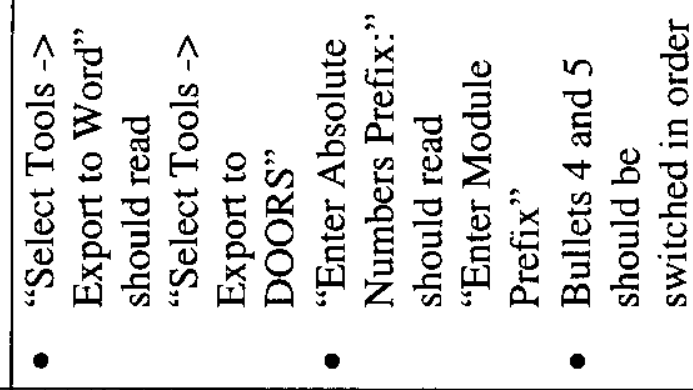 & 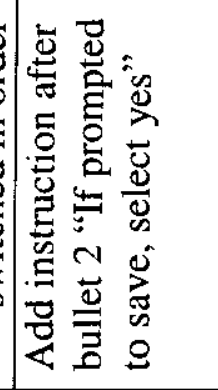 \\
\hline $\bar{\sigma}$ & & & & & & \\
\hline 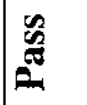 & & |\rangle & $>$ & $>$ & $>$ & $>$ \\
\hline 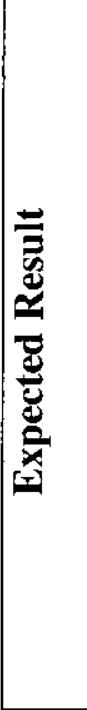 & & 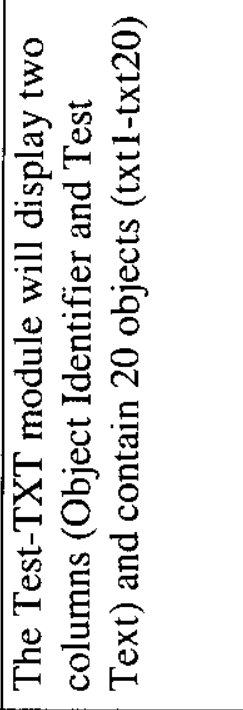 & 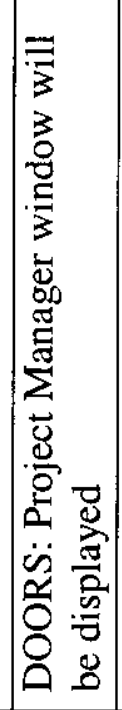 & 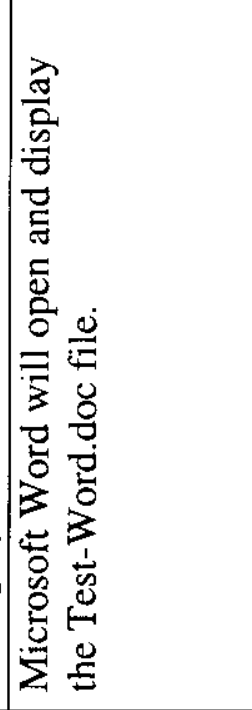 & 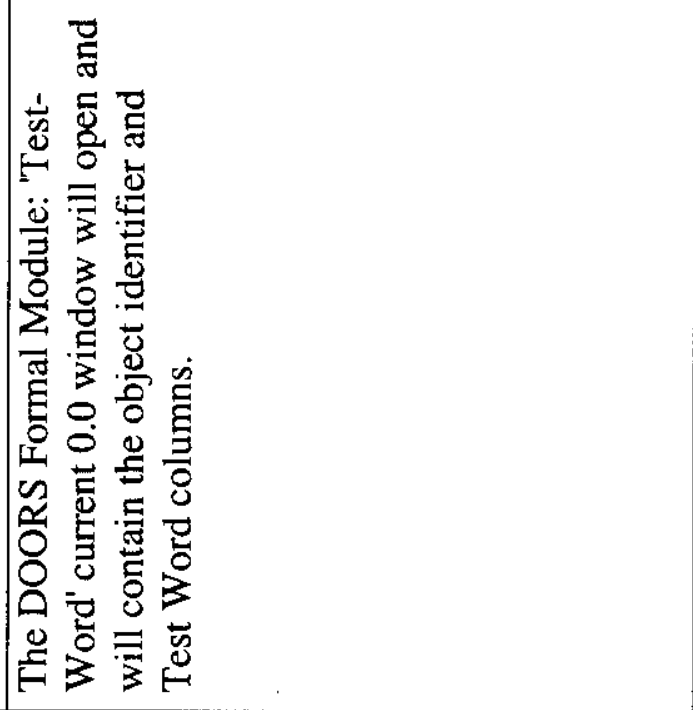 & 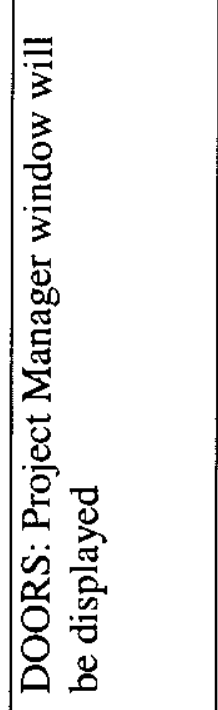 \\
\hline 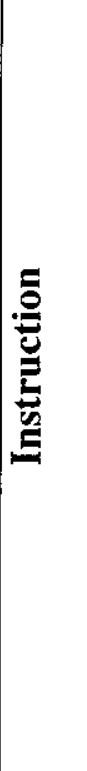 & 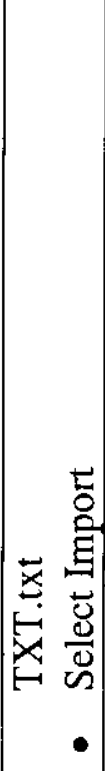 & 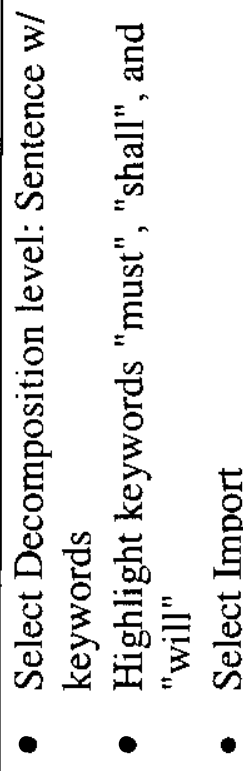 & 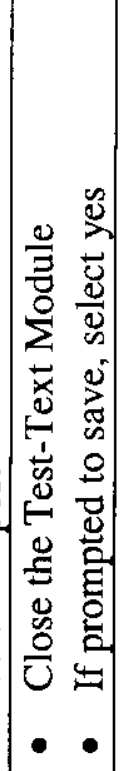 & 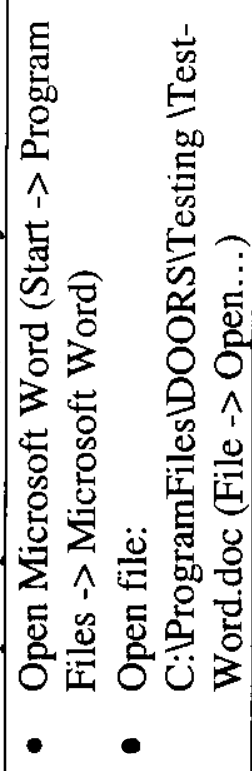 & 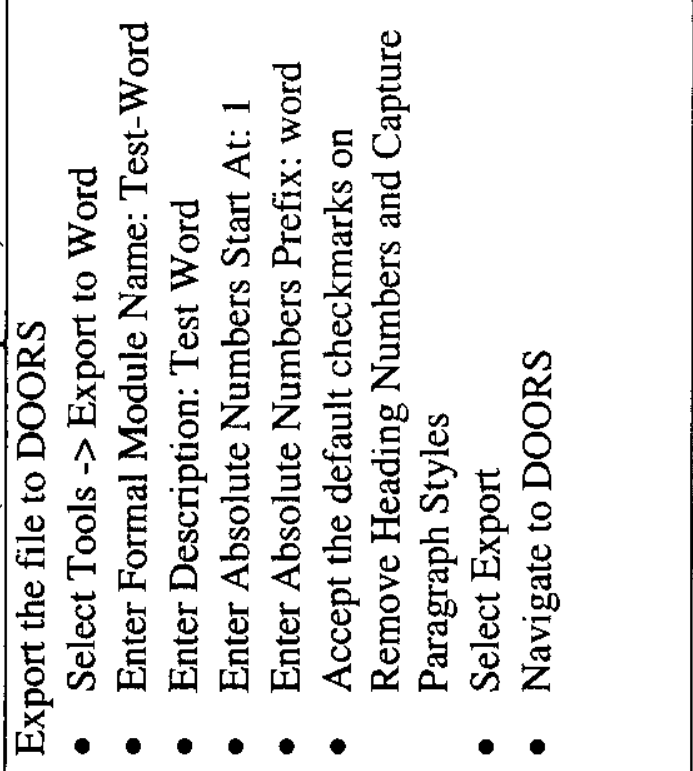 & 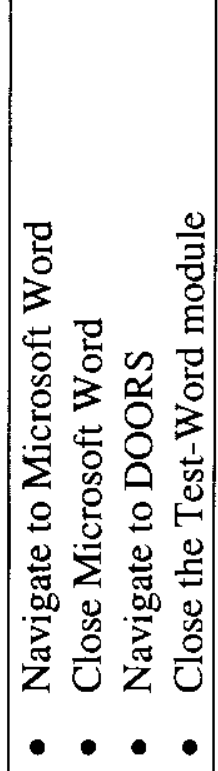 \\
\hline के & & $\Xi$ & $\stackrel{\infty}{=}$ & 2 & গু & $\vec{\sim}$ \\
\hline
\end{tabular}


RPP-6251 REV. 0

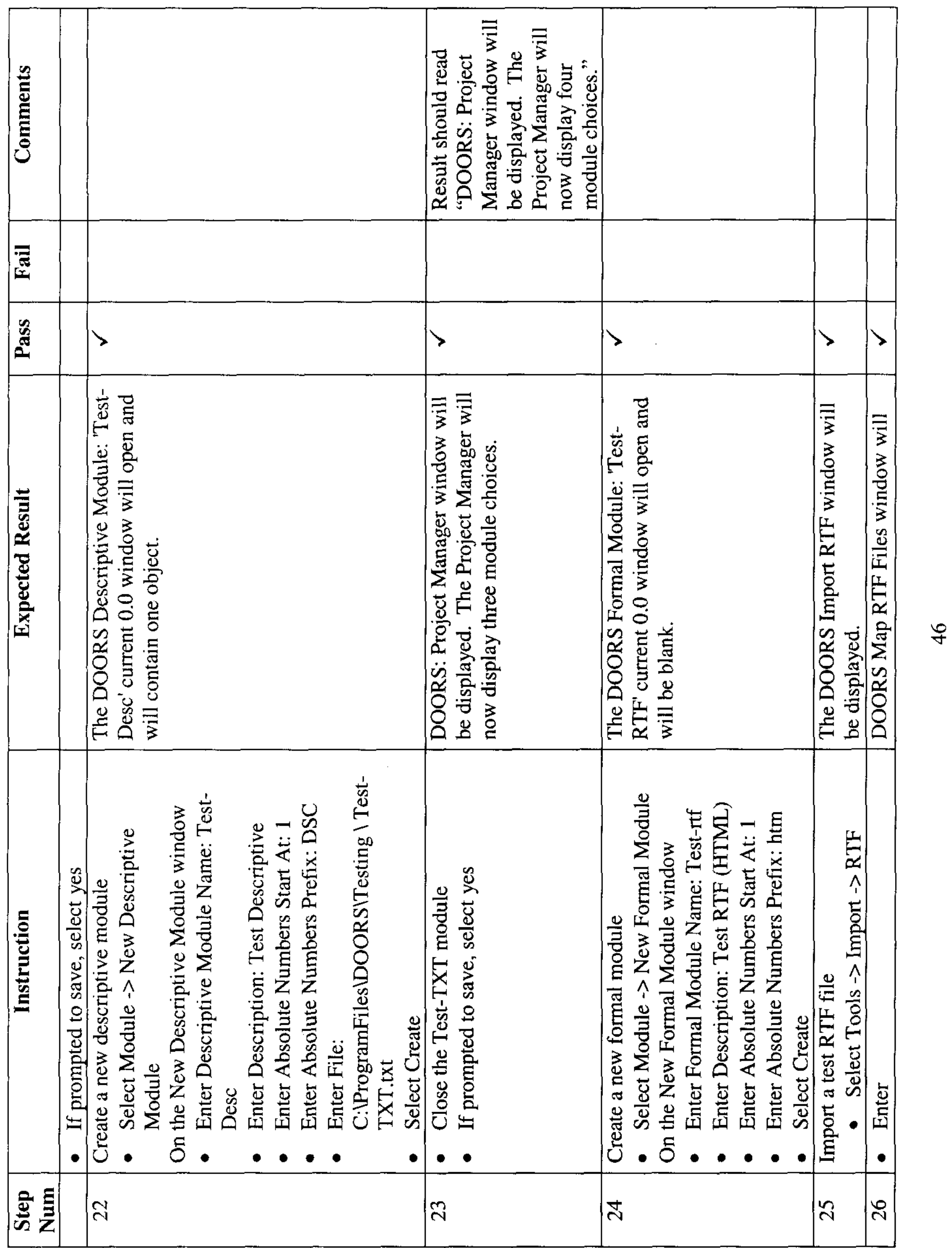


RPP-6251 REV. 0

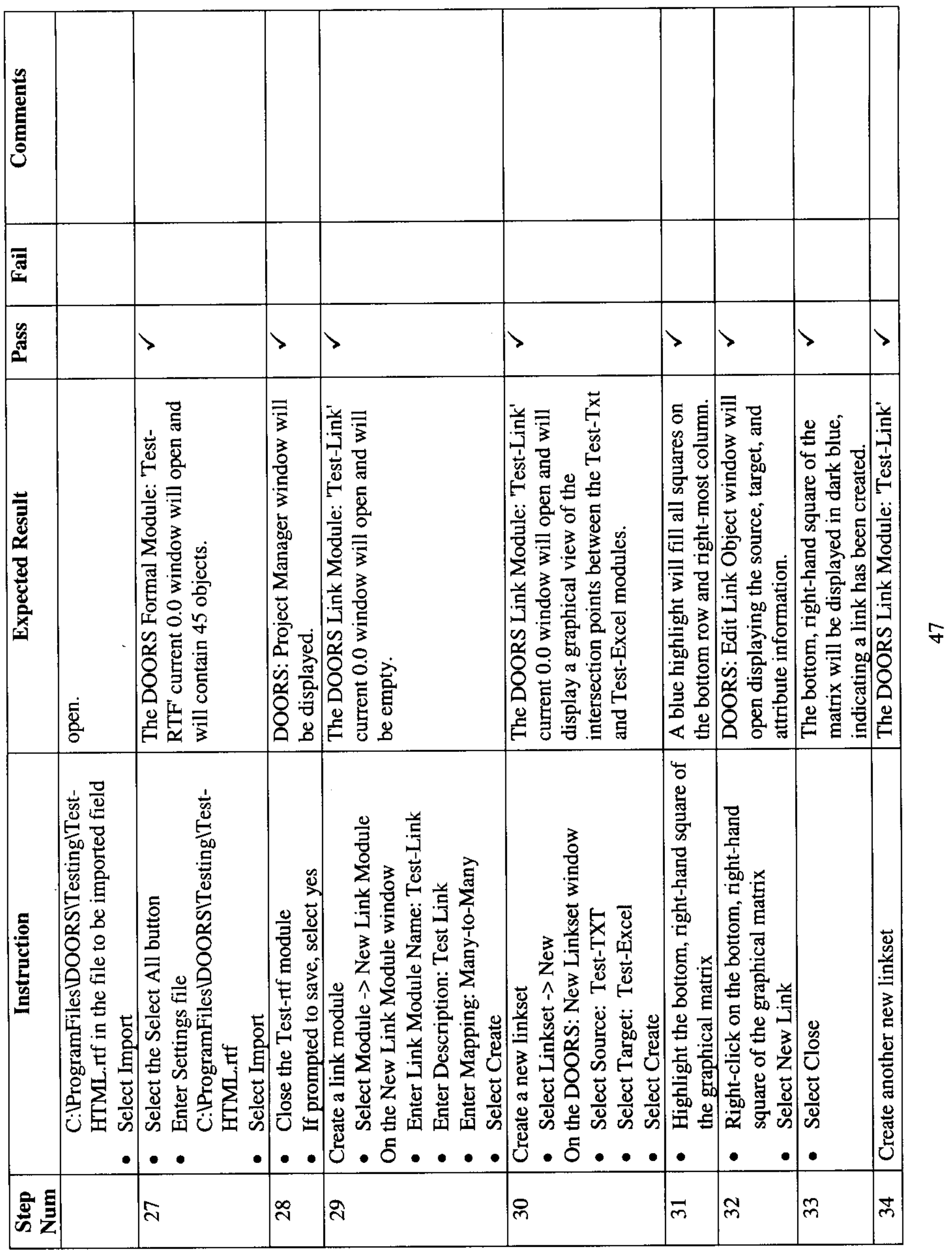


RPP-6251 REV. 0

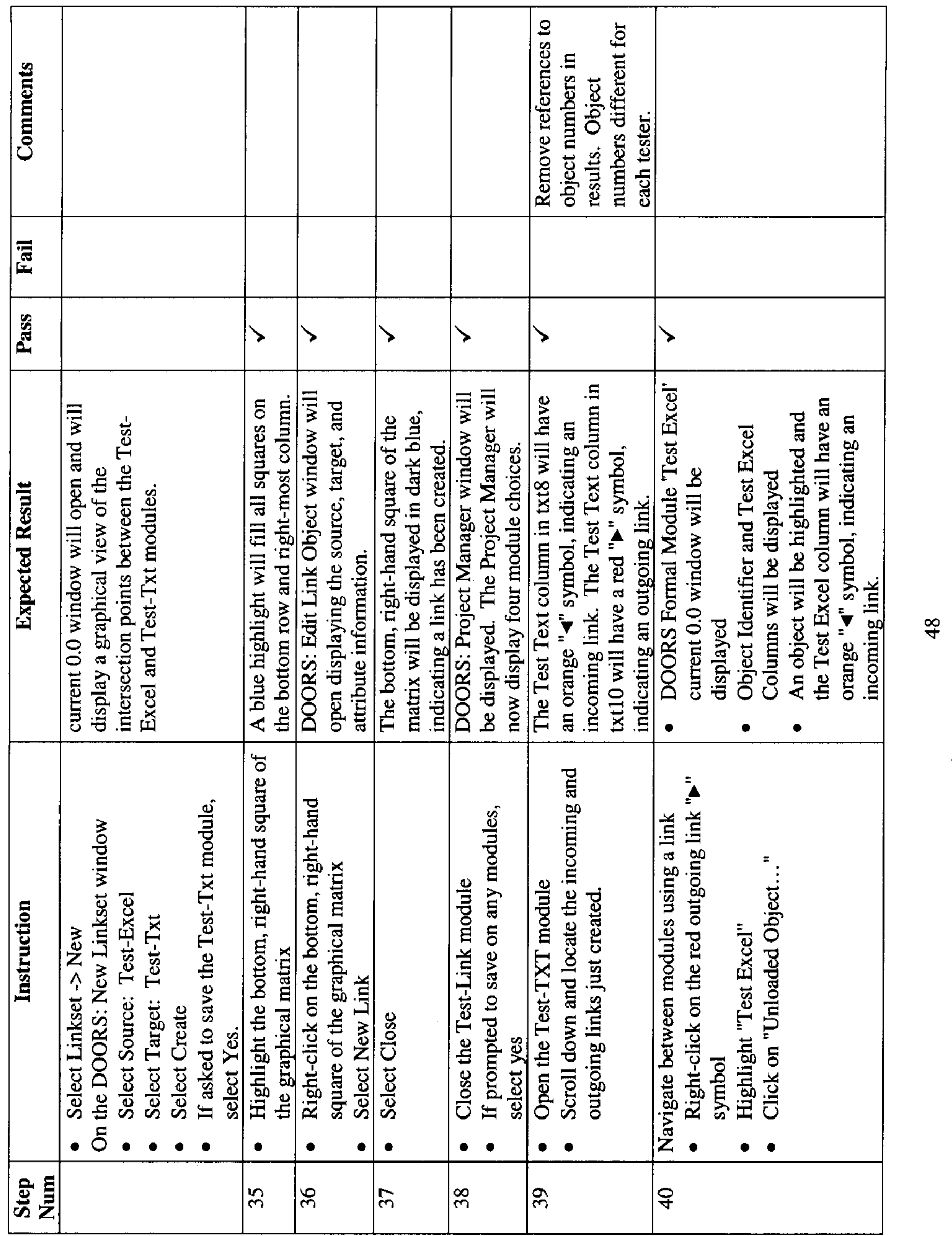




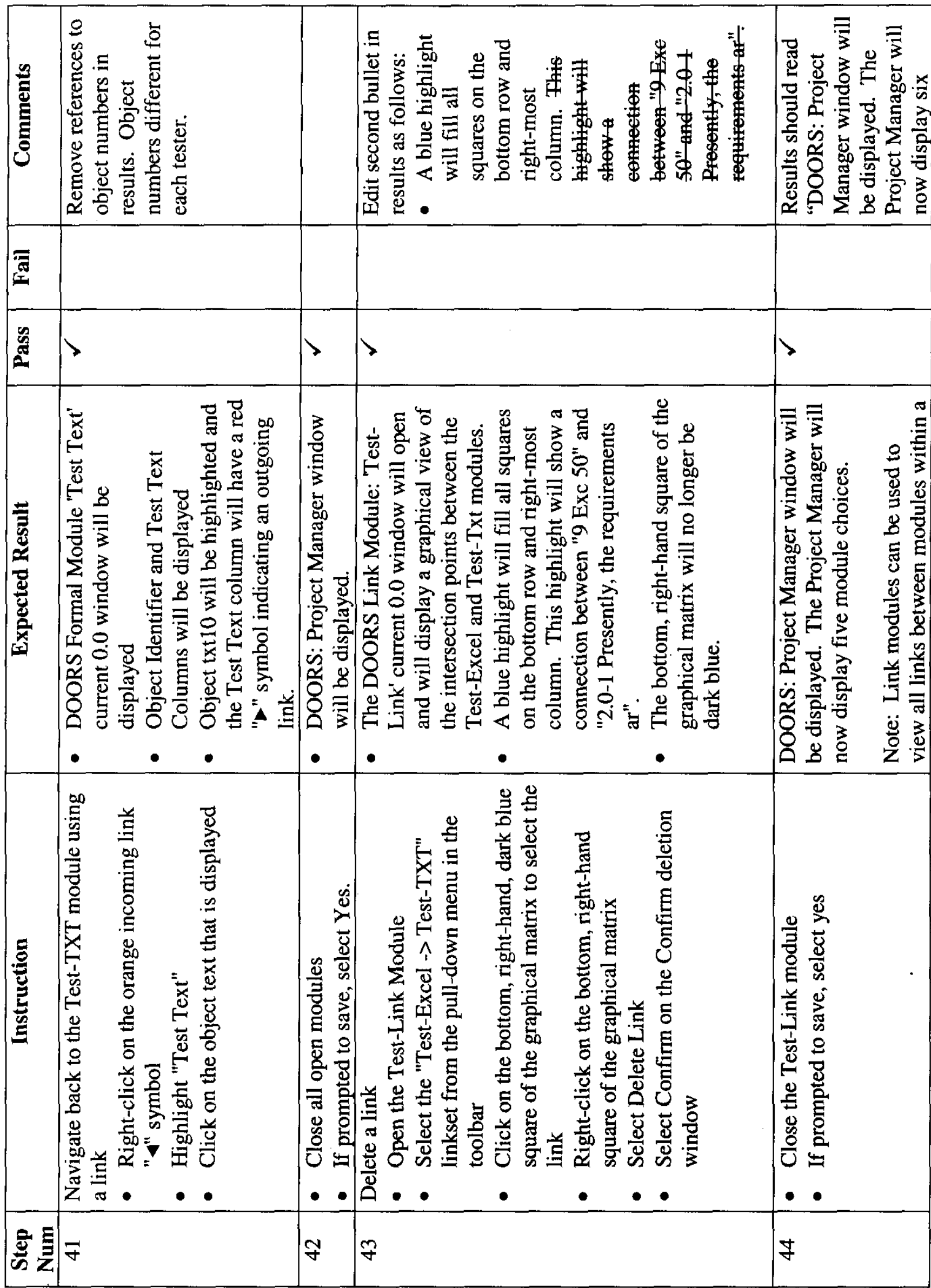


RPP-6251 REV. 0

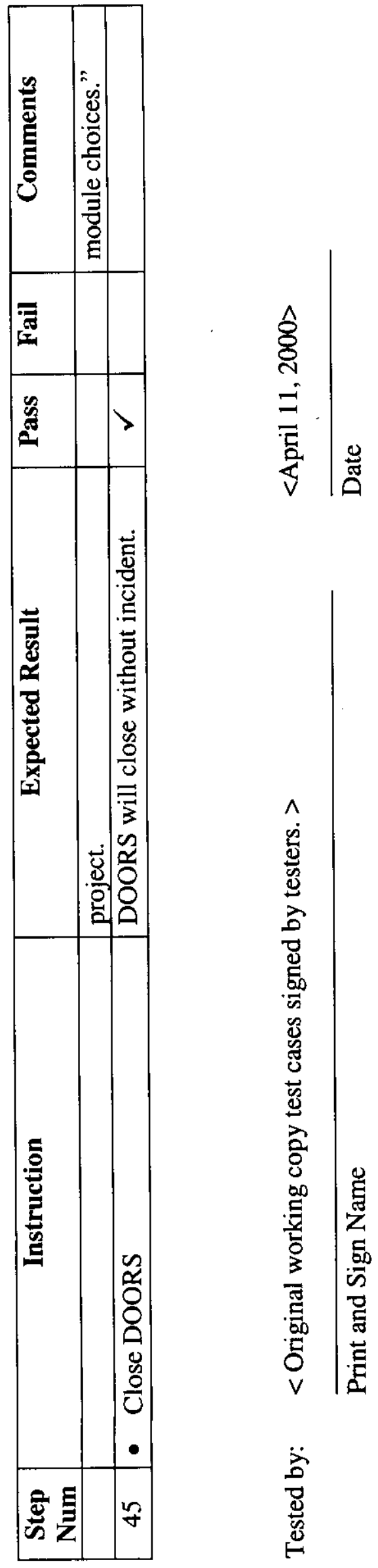




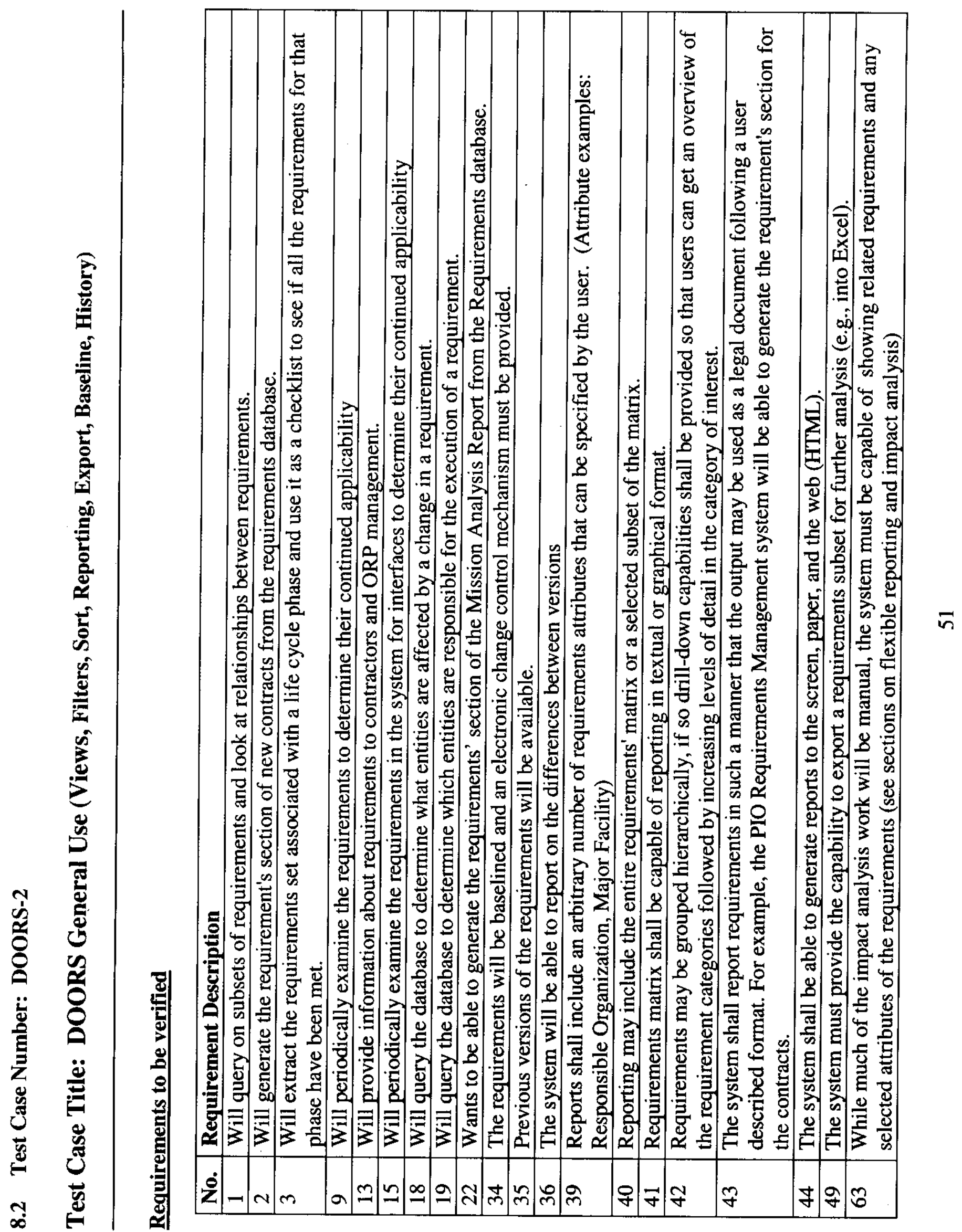



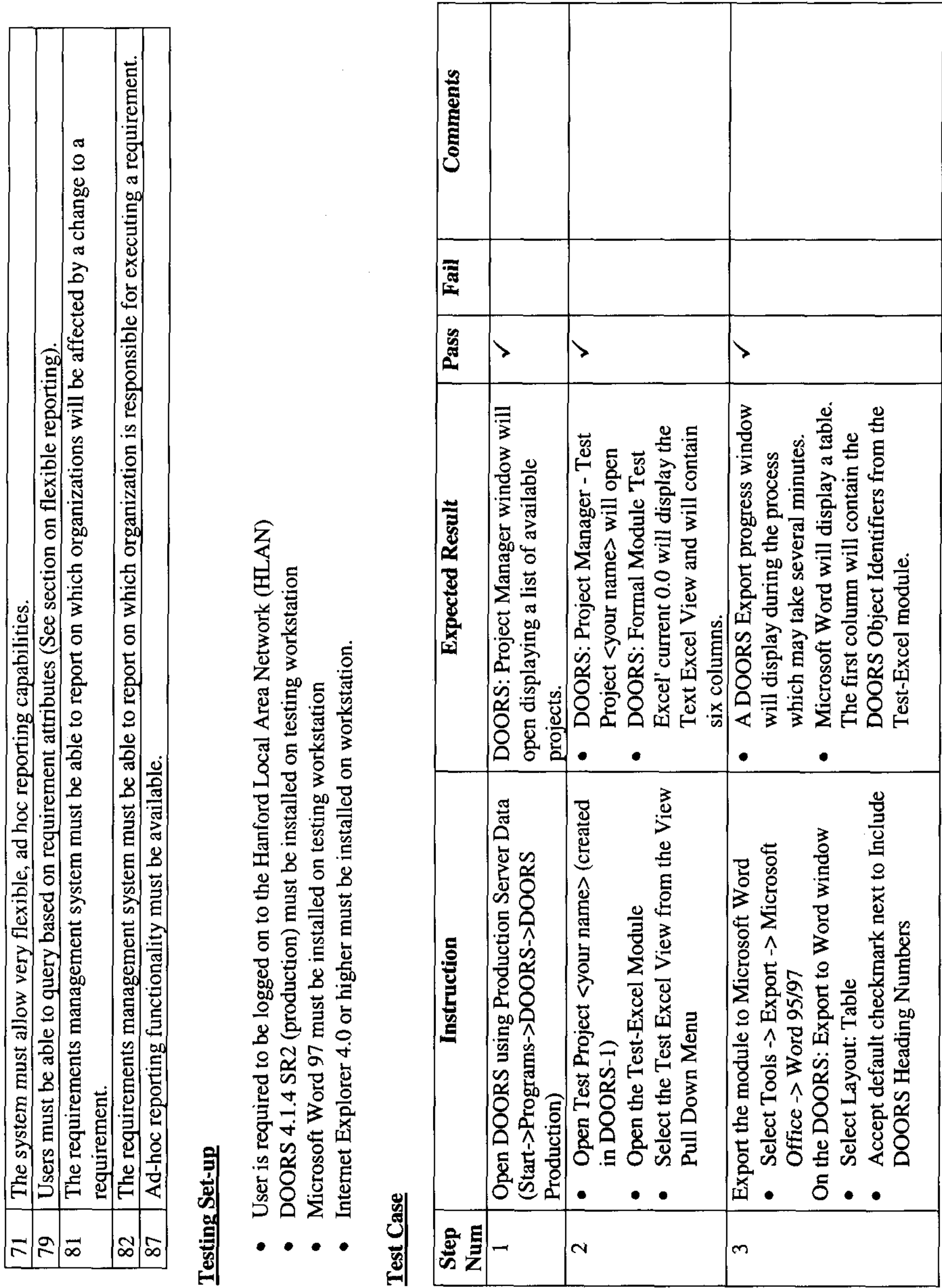
RPP-6251 REV. 0

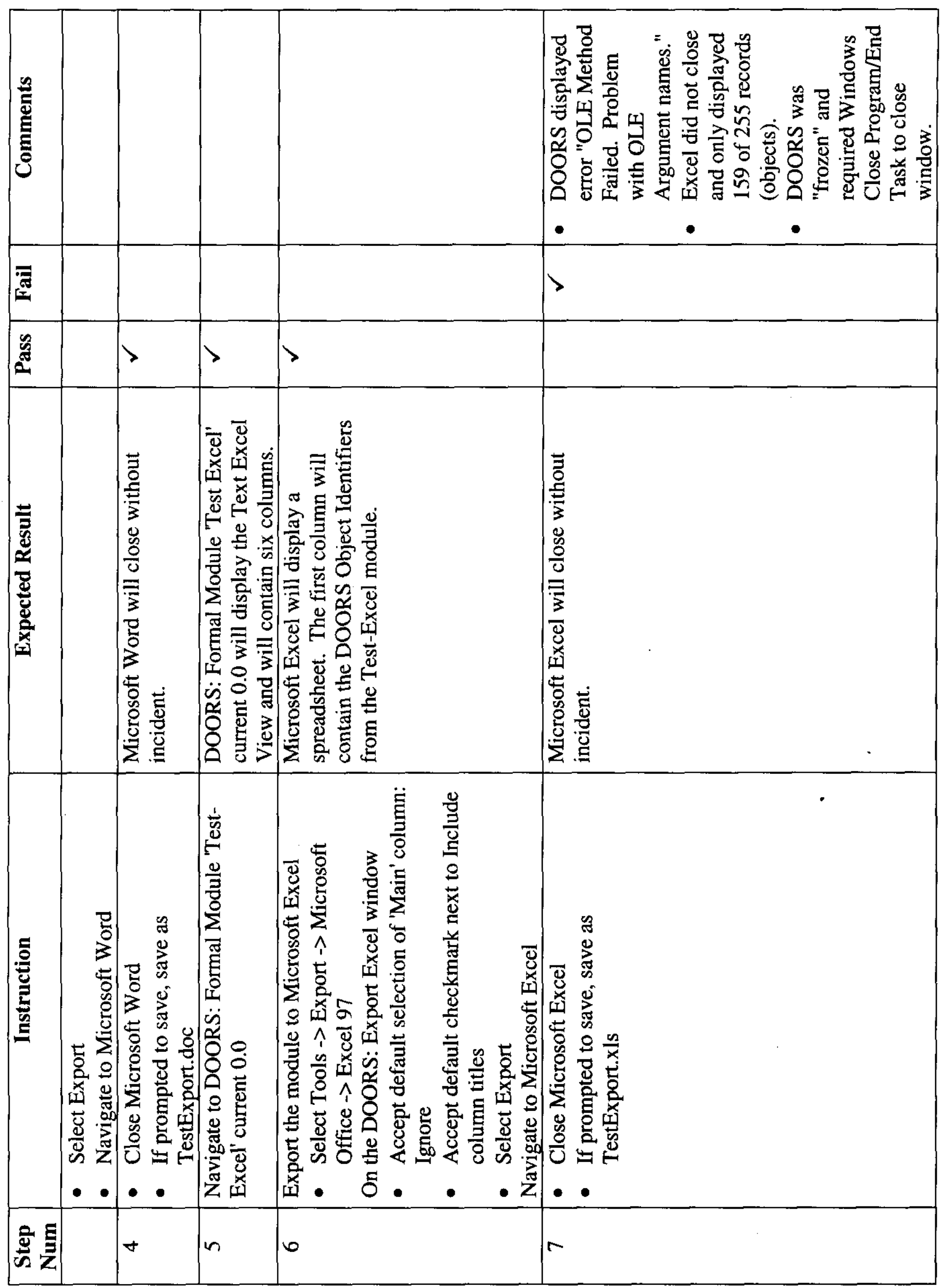


RPP-6251 REV. 0

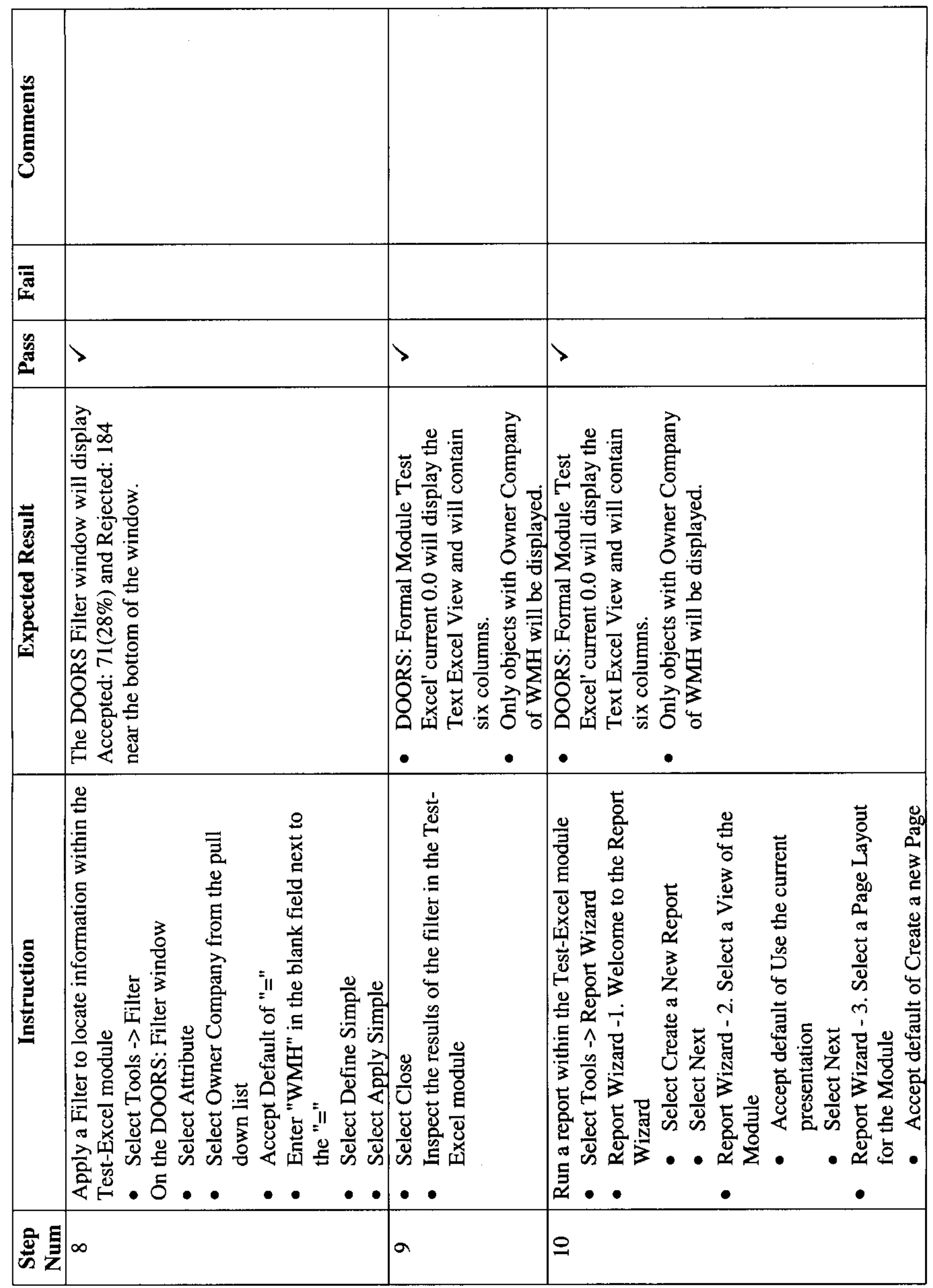


RPP-6251 REV. 0

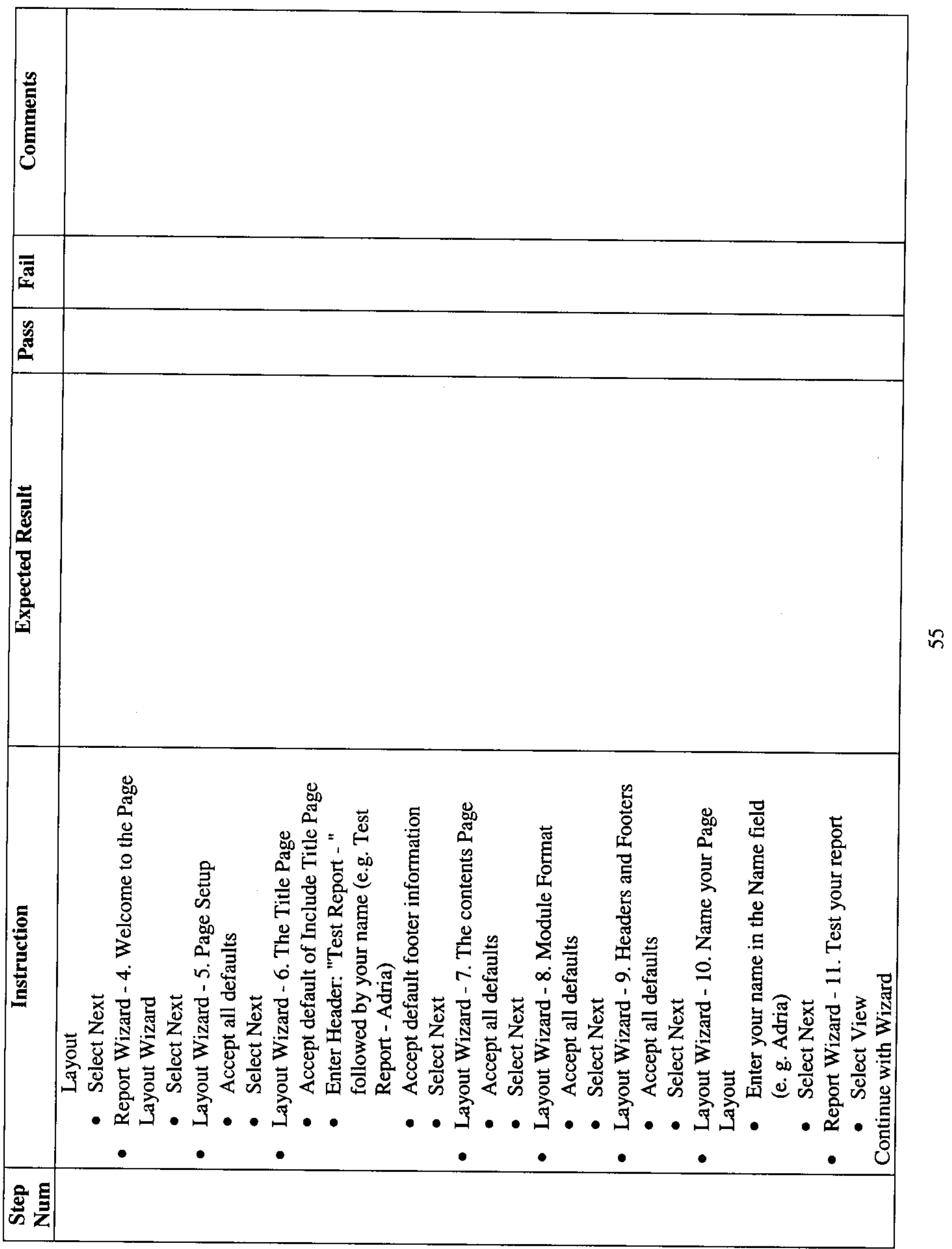


RPP-6251 REV. 0

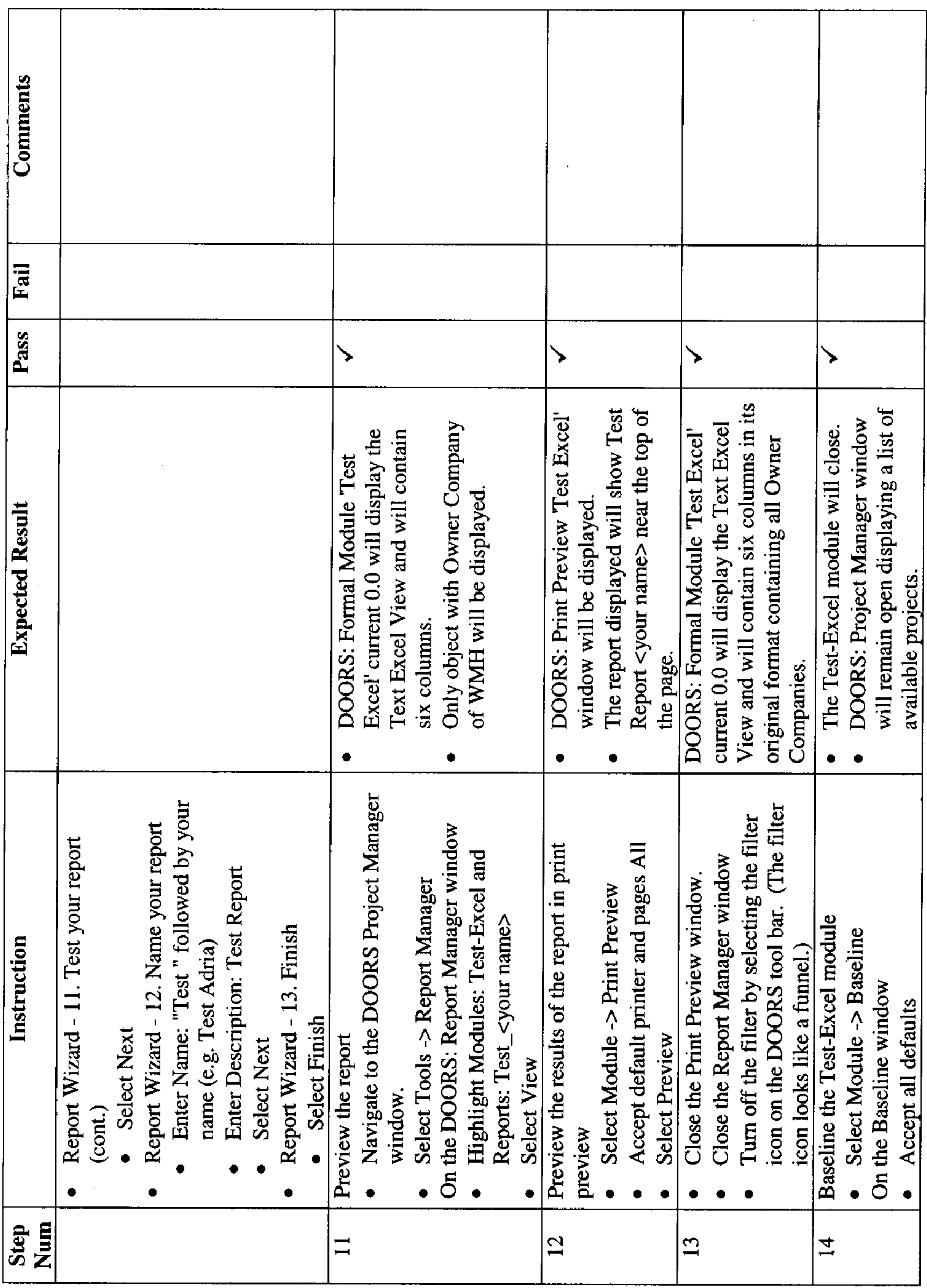


RPP-6251 REV. 0

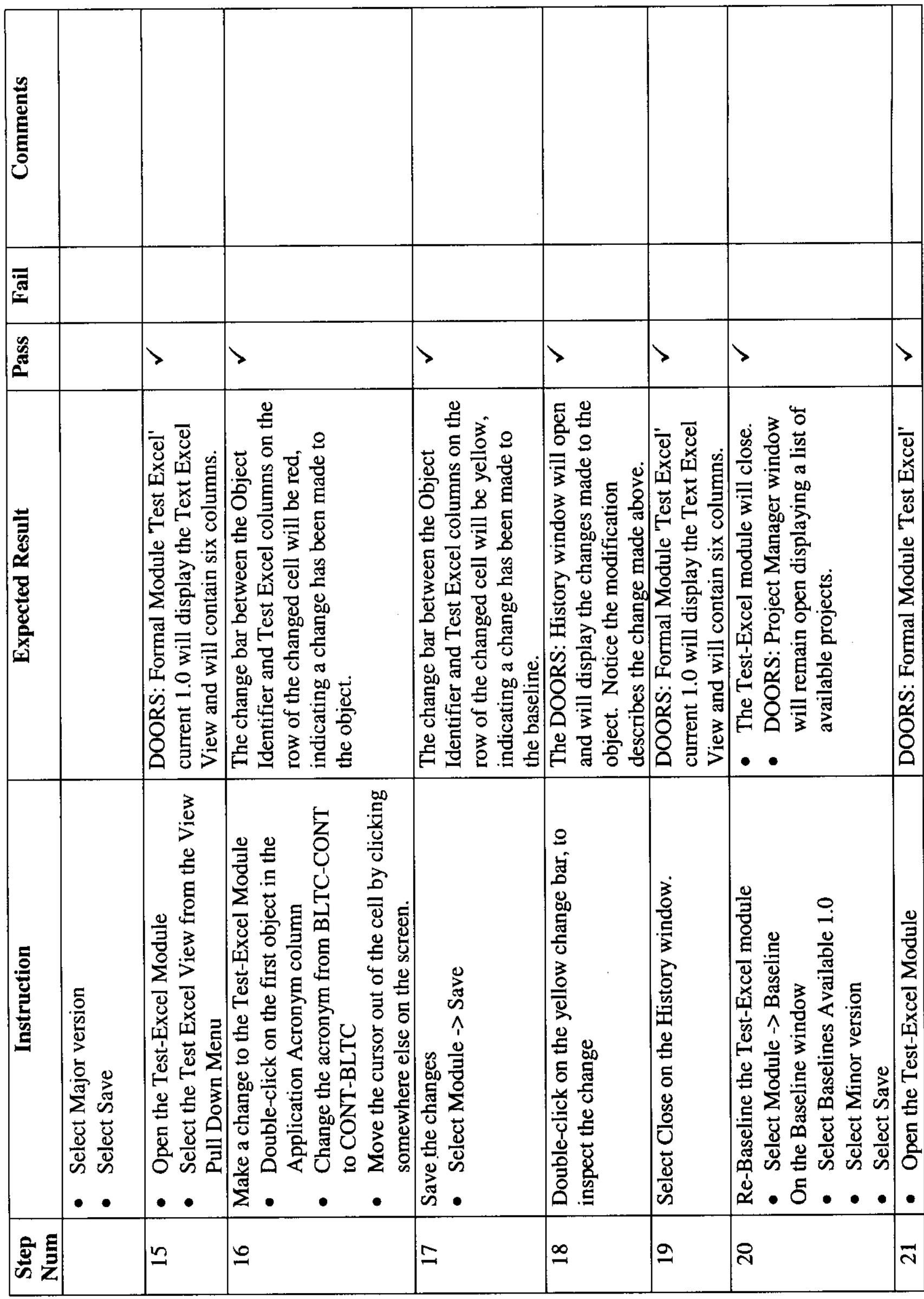


RPP-6251 REV. 0

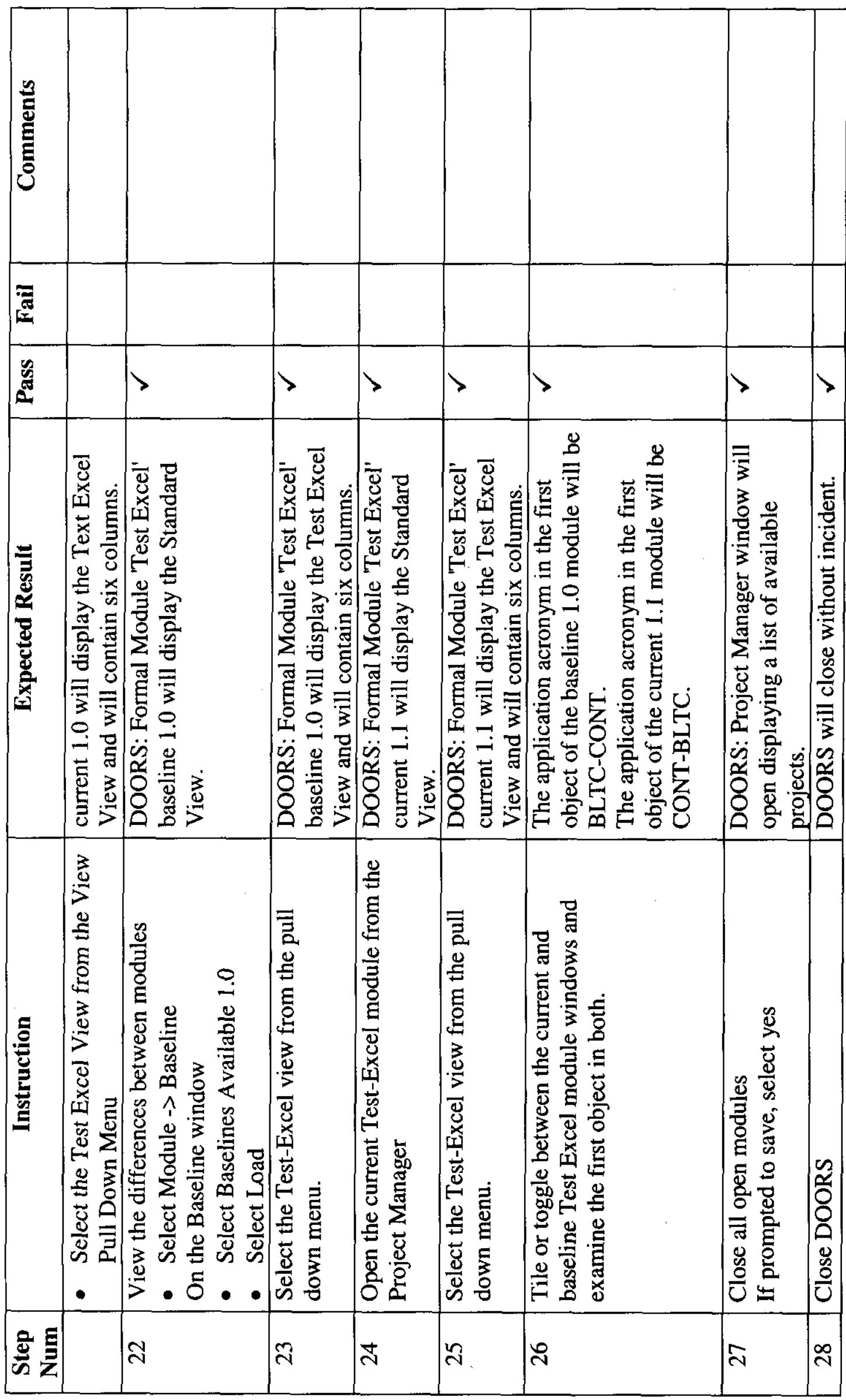
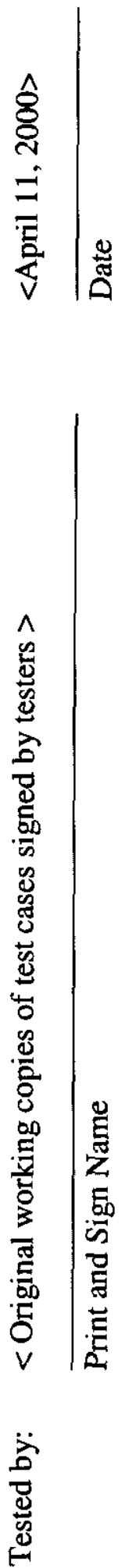
RPP-6251 REV. 0
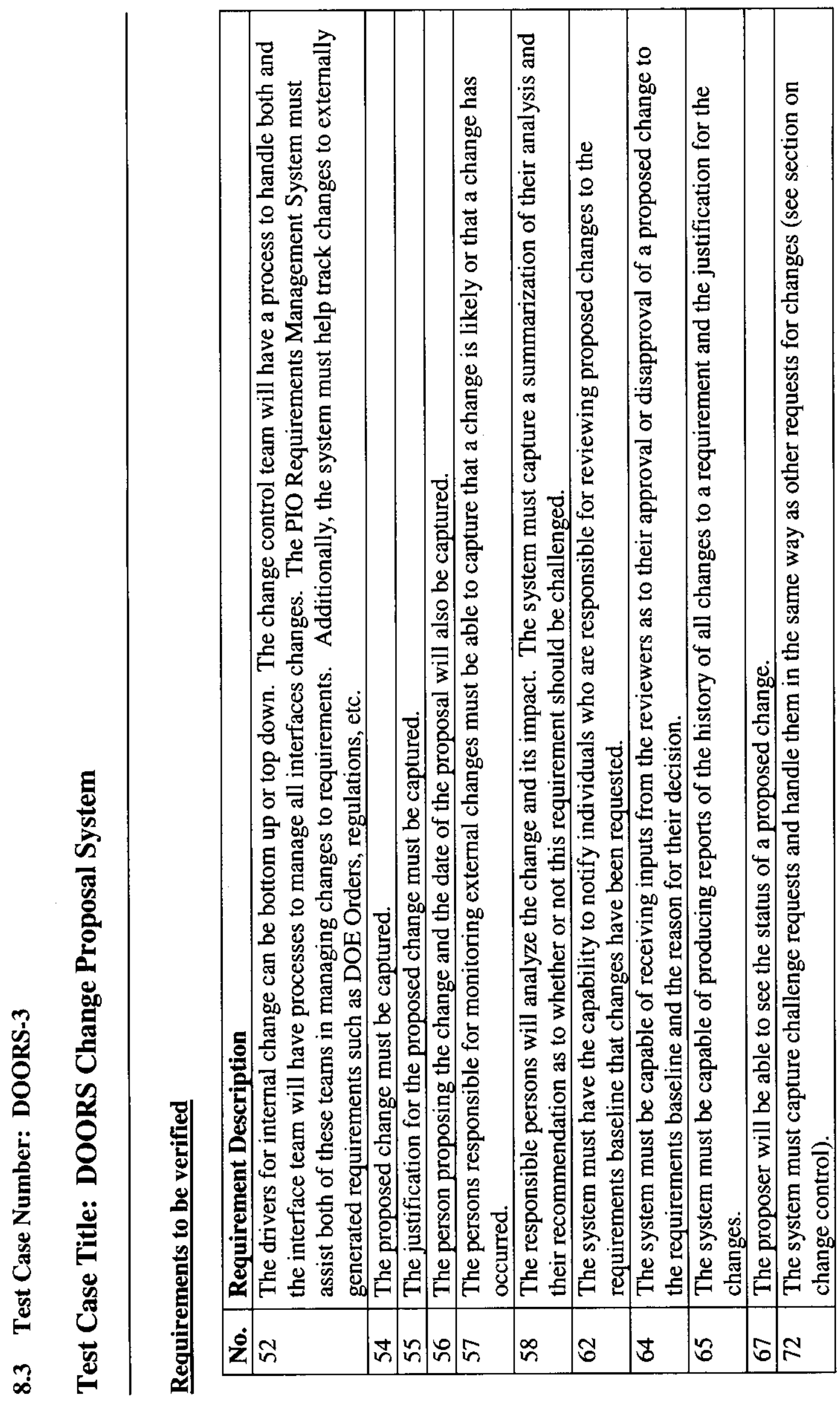

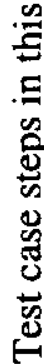

之्己

를

응

范

E

\&

¿

8 空

$\circ 5$

当

屯.

Z

농

8.

$\checkmark$

\%

$8=$

를

ช్․ㅀ

क

灾芯

สี

\&

定

品

范

ठ 8

o 5 . 
RPP-6251 REV. 0
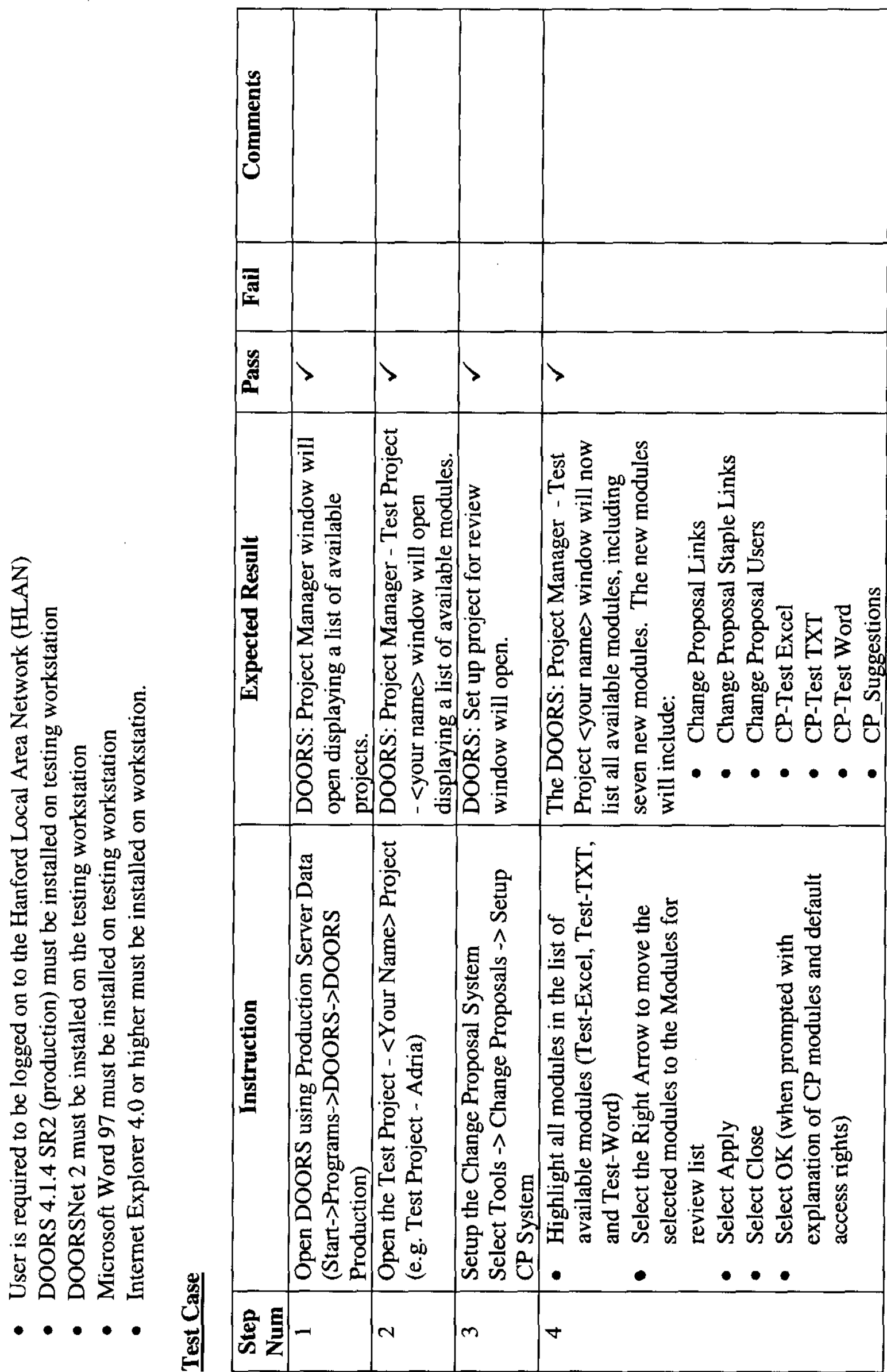

8 
RPP-6251 REV. 0

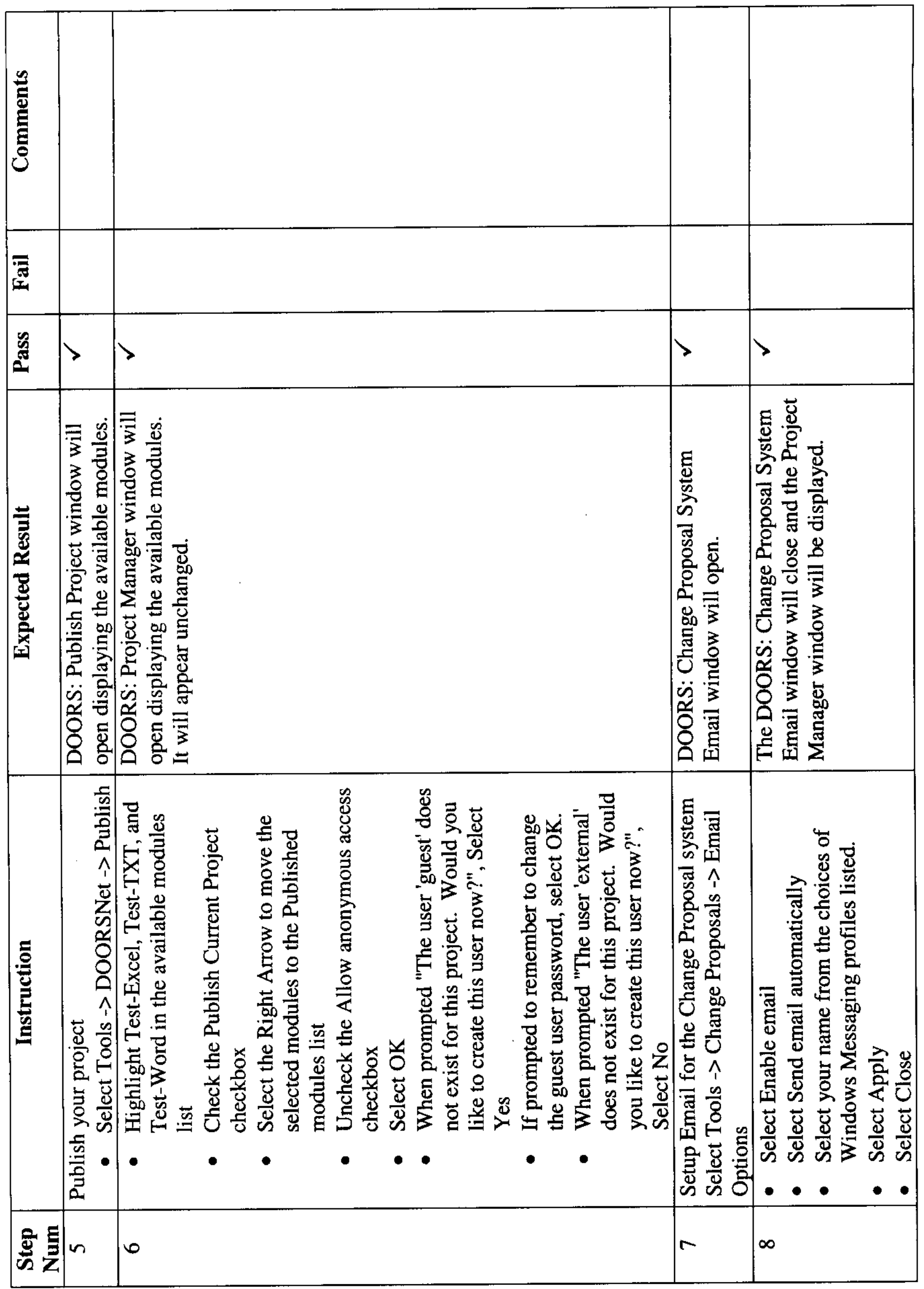




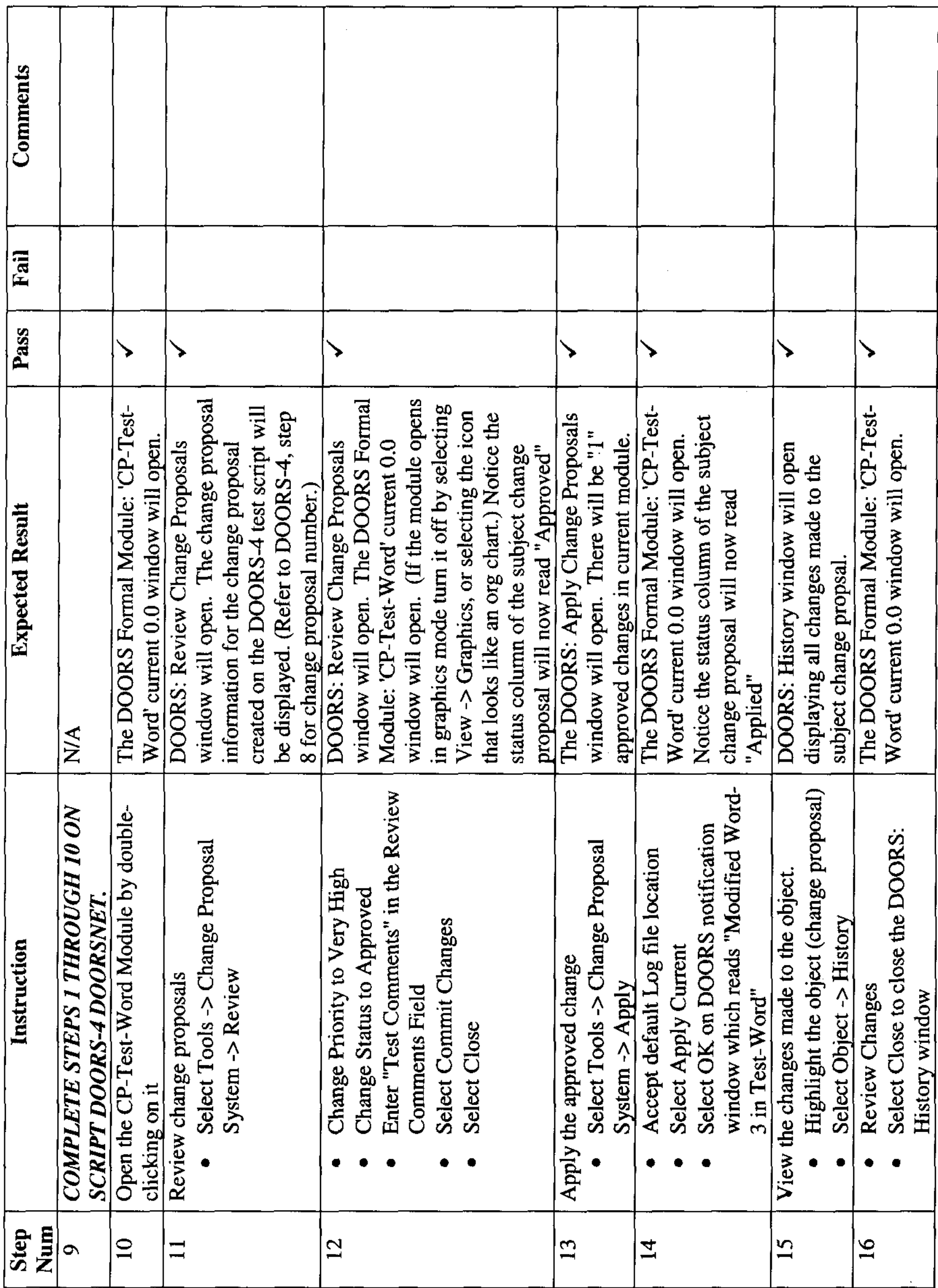


RPP-6251 REV. 0

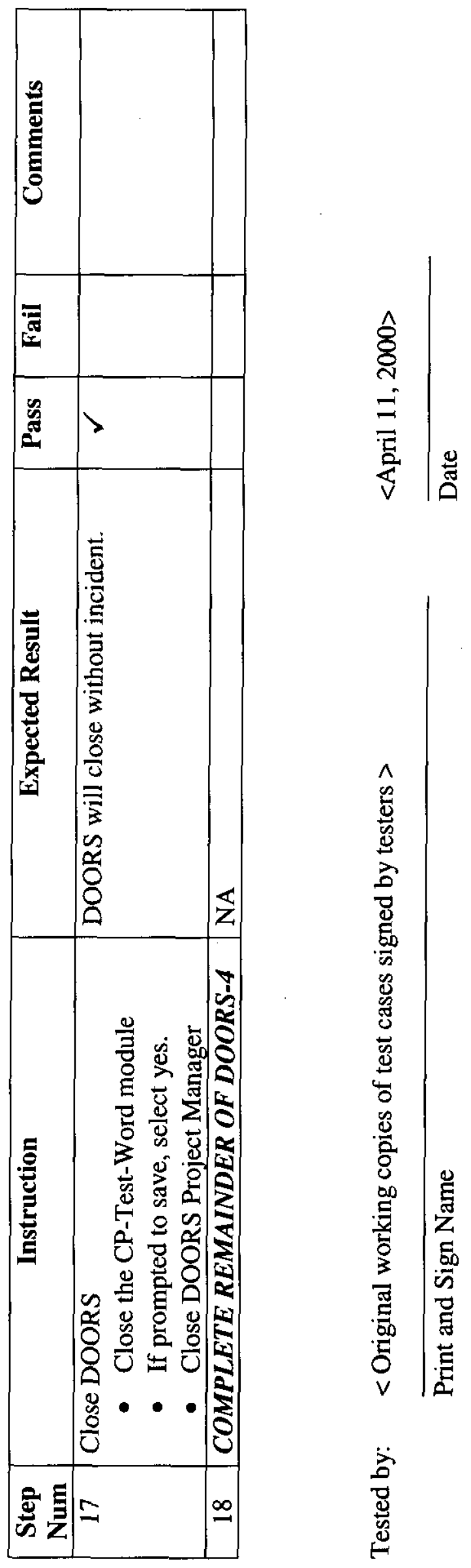


RPP-6251 REV. 0
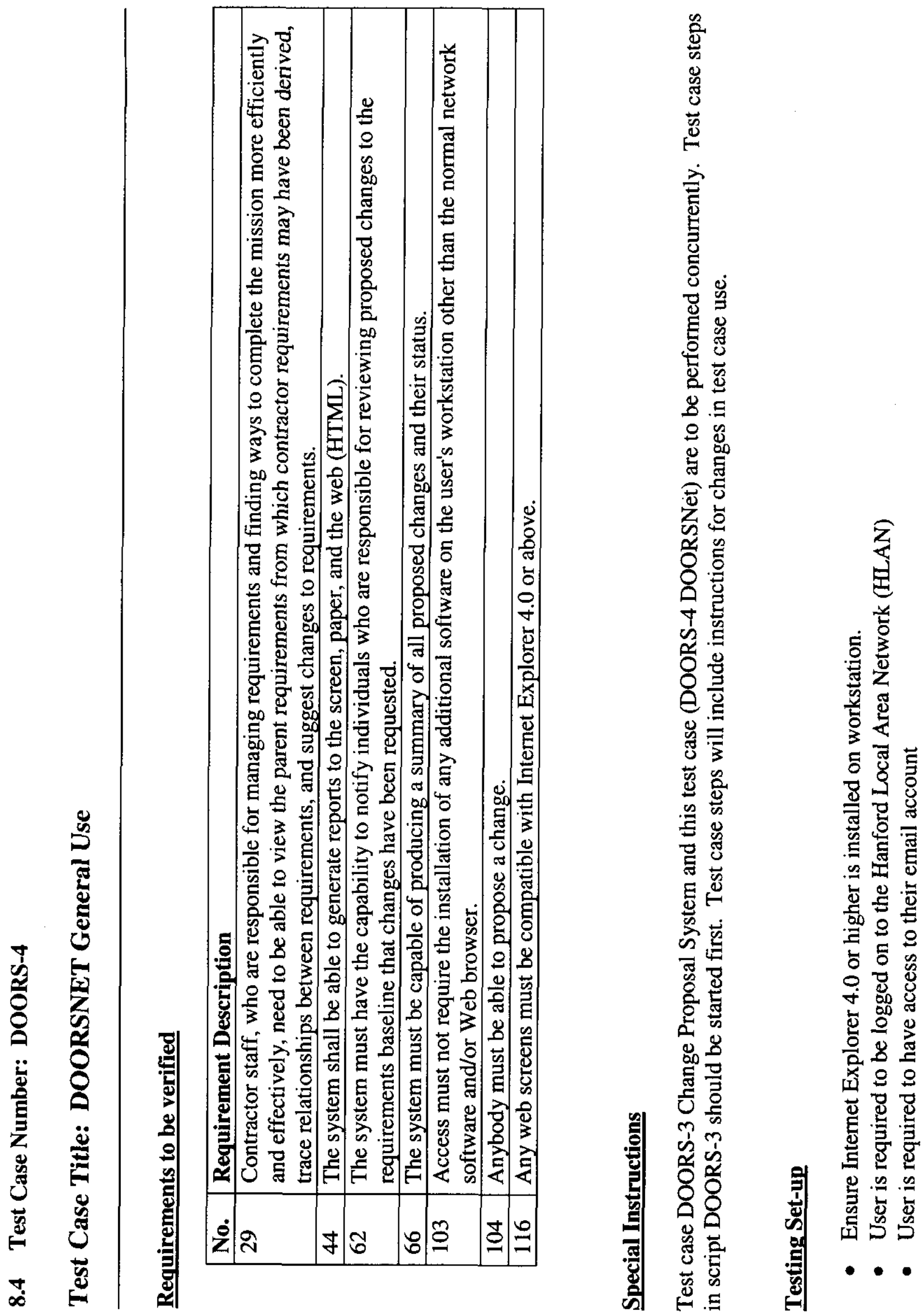
RPP-6251 REV. 0

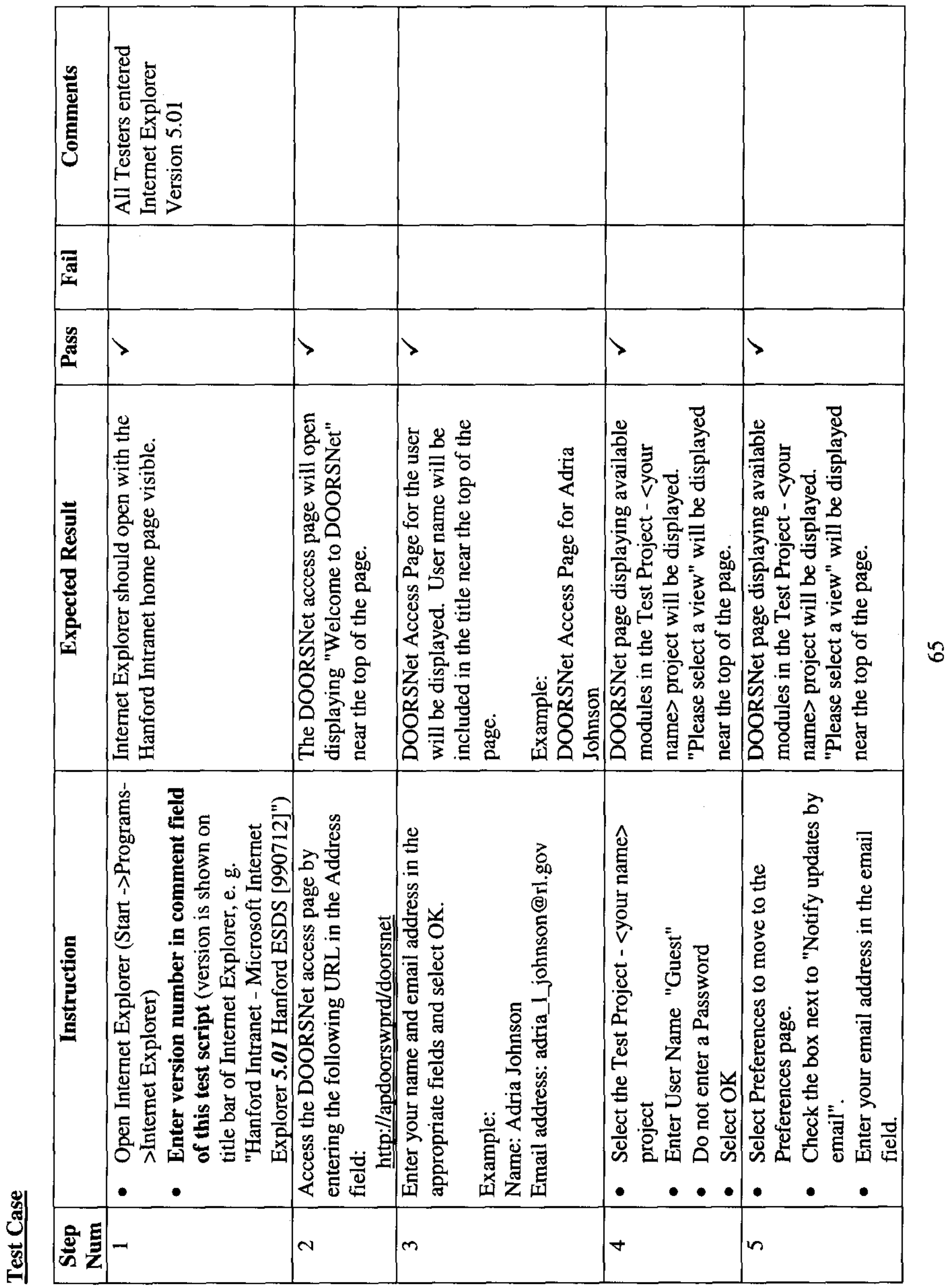


RPP-6251 REV. 0

\begin{tabular}{|c|c|c|c|c|c|c|}
\hline 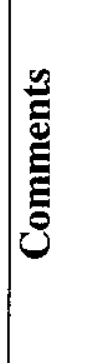 & & & & 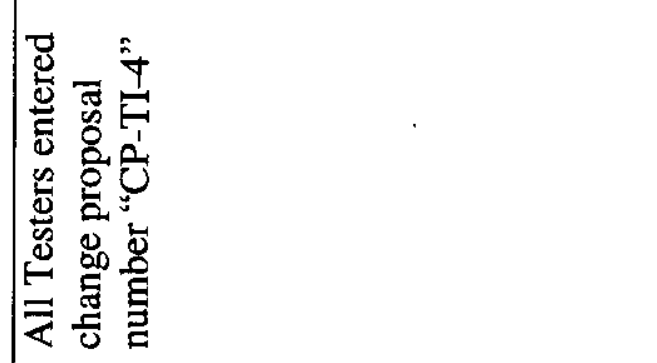 & & \\
\hline ت & & & & & & \\
\hline 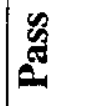 & & $>$ & $>$ & $>$ & $>$ & $>$ \\
\hline 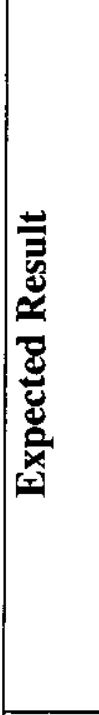 & & 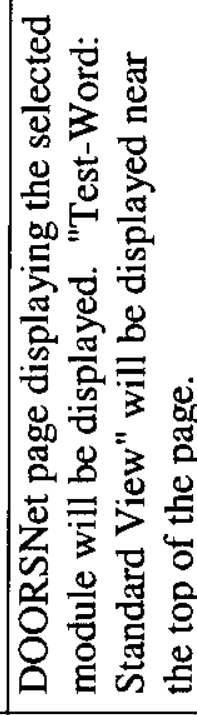 & 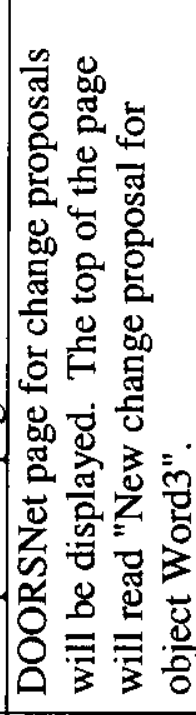 & 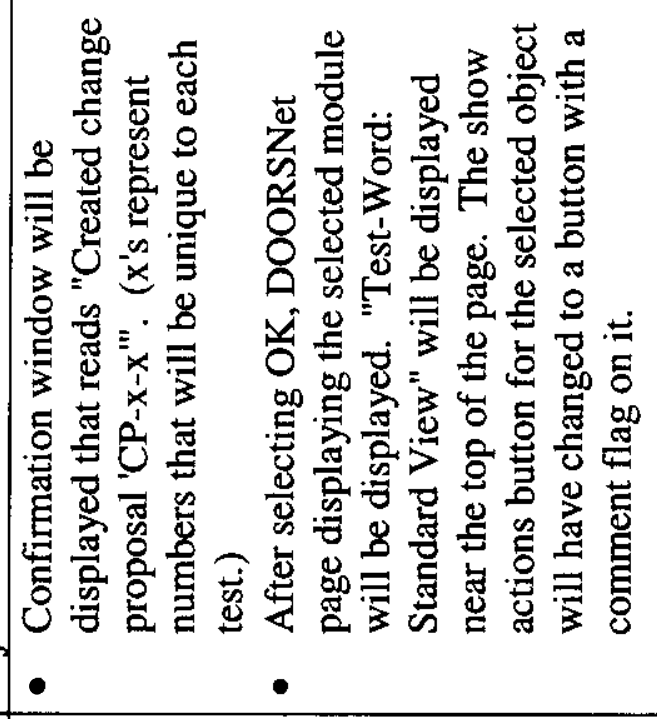 & 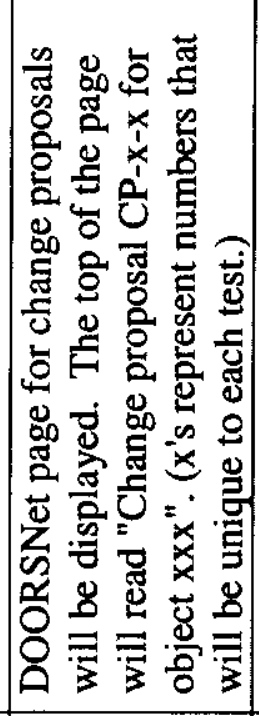 & 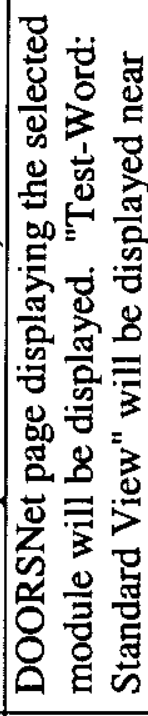 \\
\hline 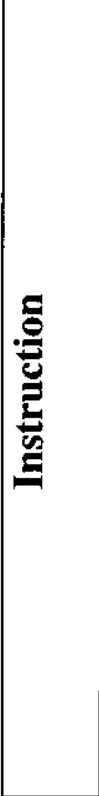 & 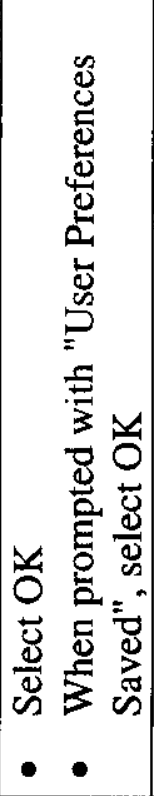 & 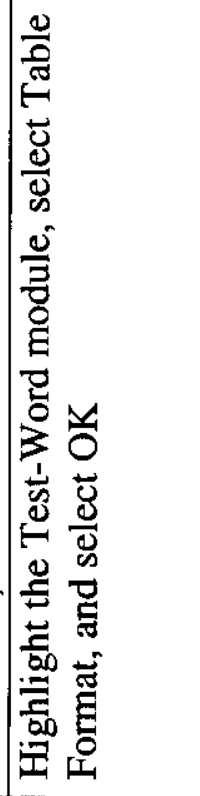 & 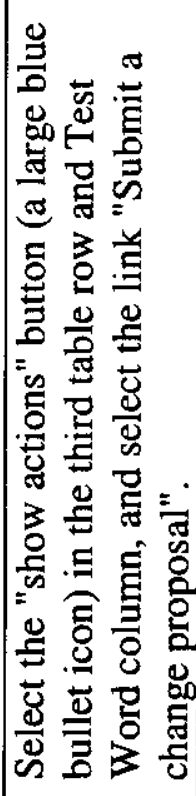 & 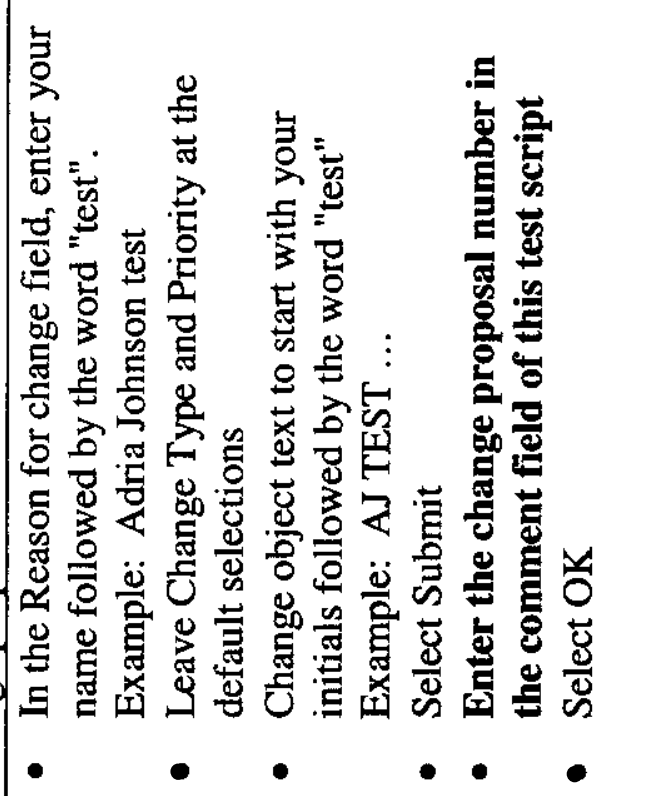 & 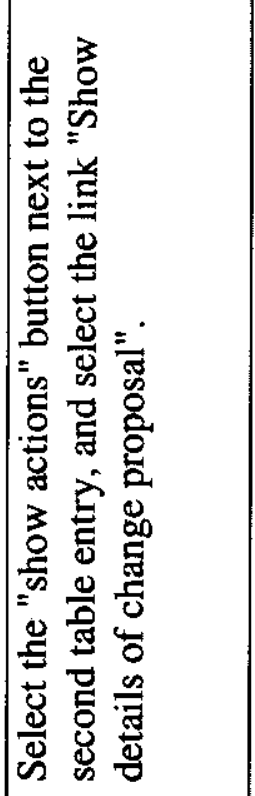 & 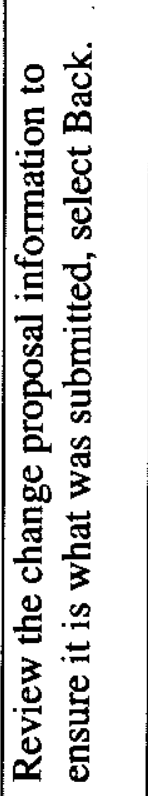 \\
\hline$\frac{0}{5}$ & & 10 & $r$ & $\infty$ & $a$ & 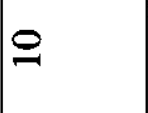 \\
\hline
\end{tabular}


RPP-6251 REV. 0

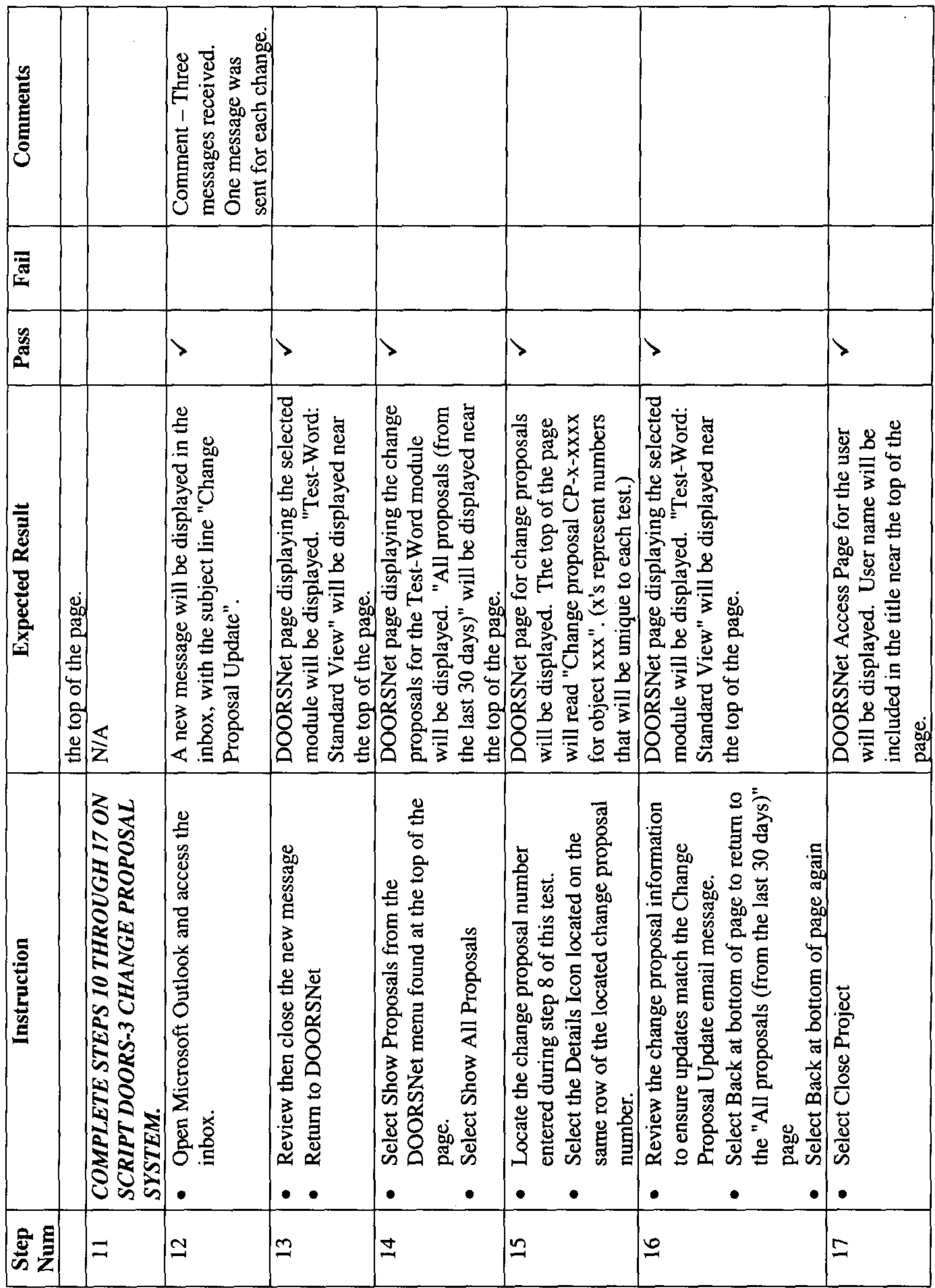


RPP-6251 REV. 0

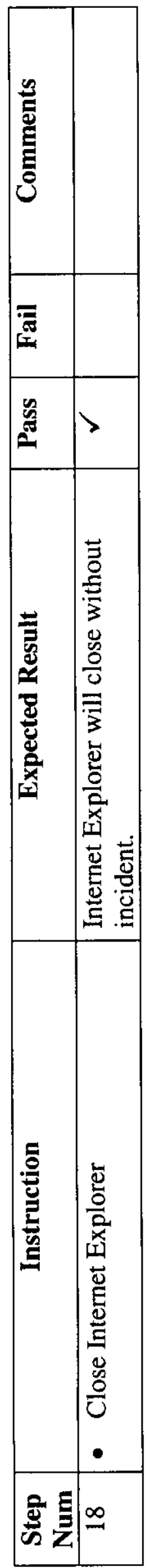

㫘

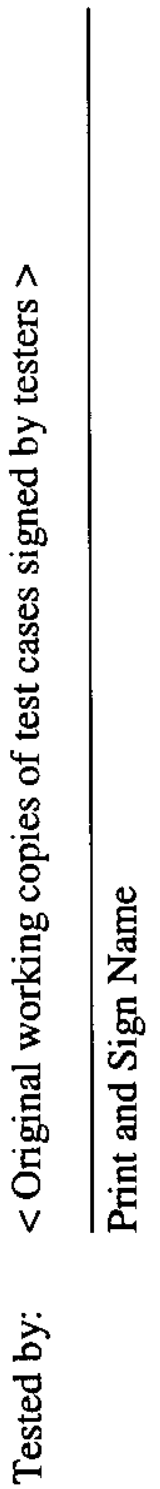


RPP-6251 REV. 0

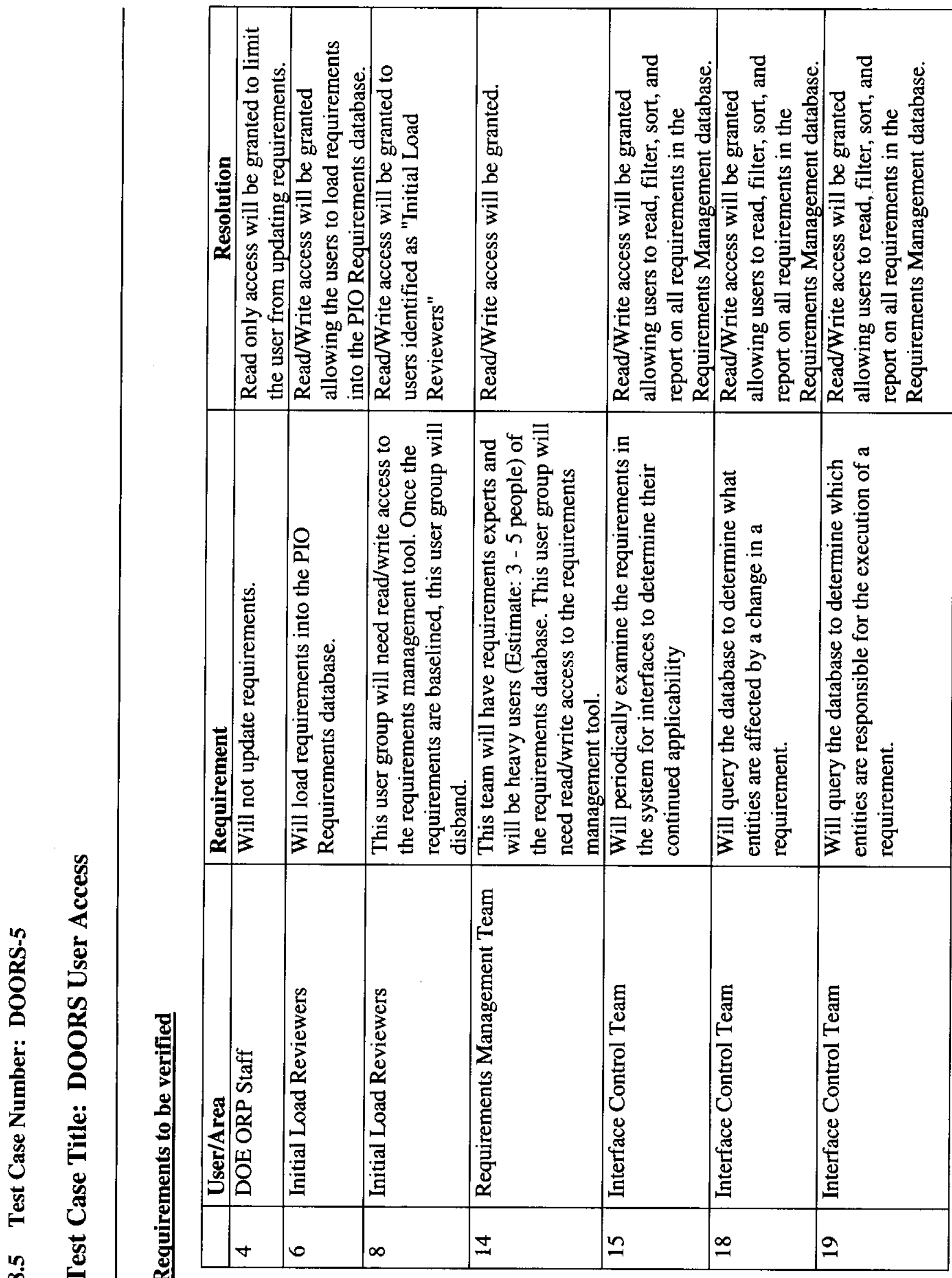




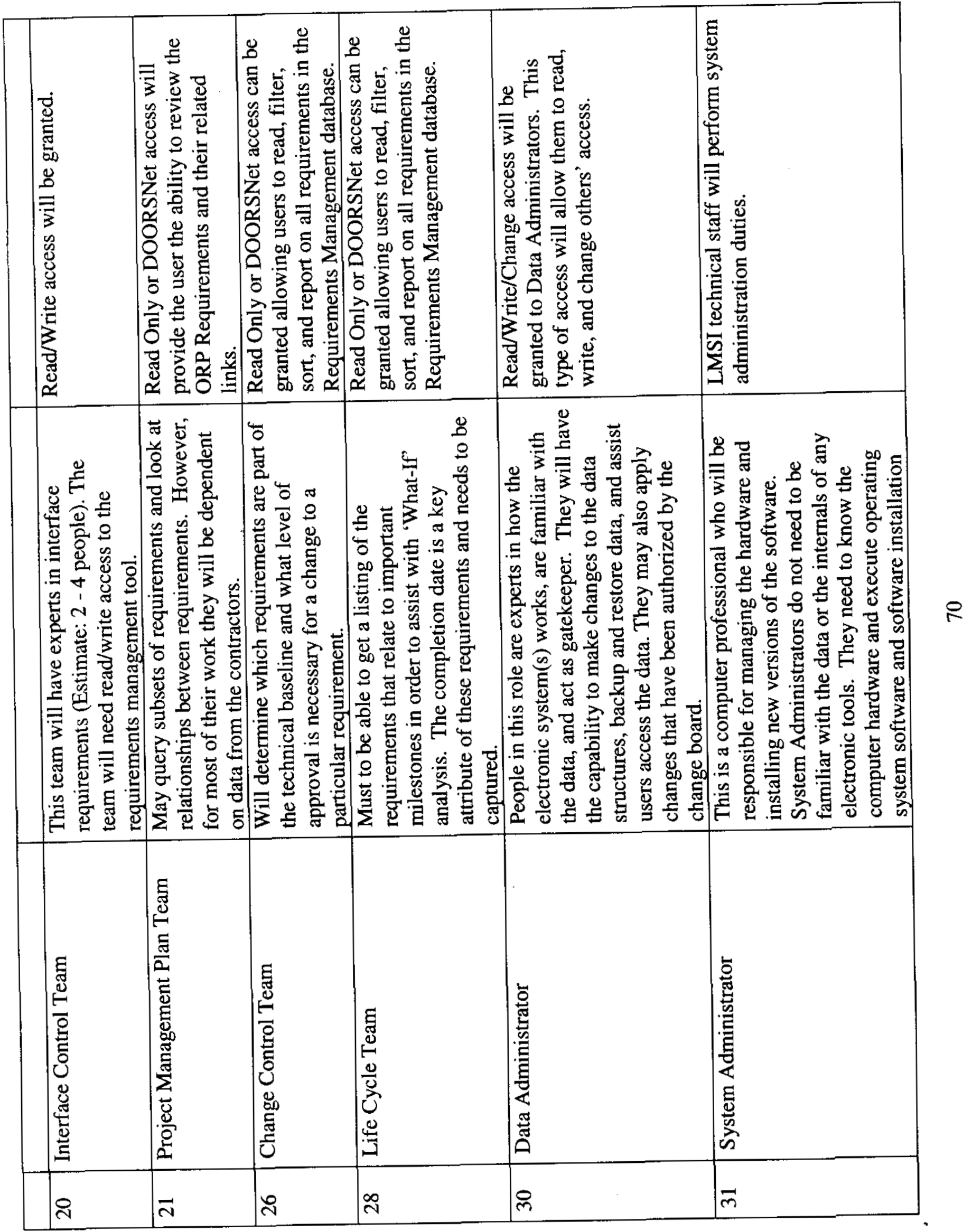


RPP-6251 REV. 0

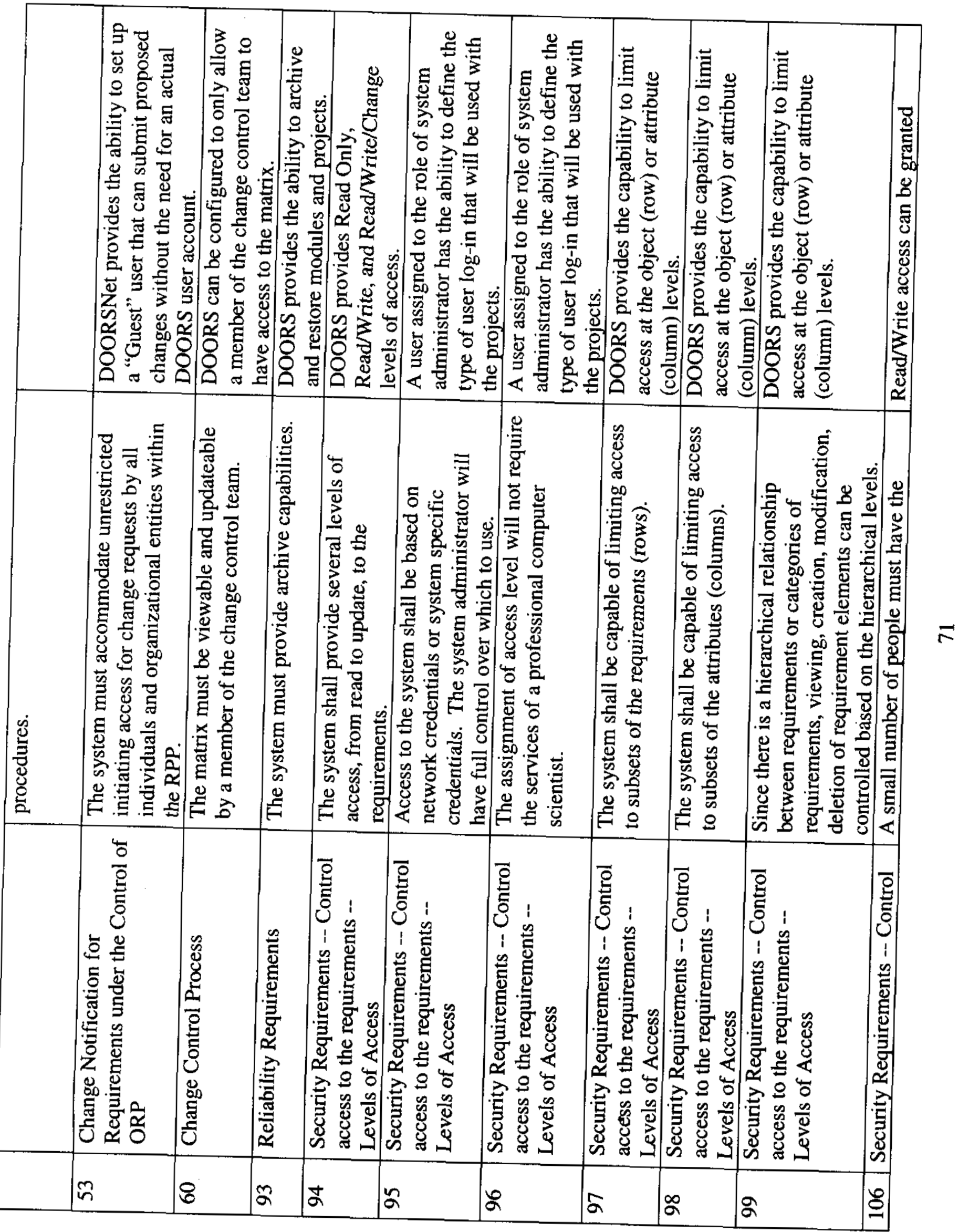


RPP-6251 REV. 0

\begin{tabular}{|c|c|c|}
\hline 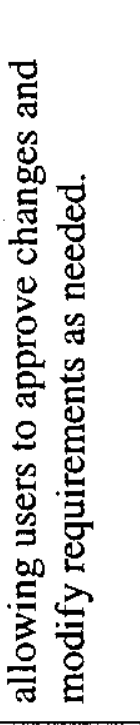 & 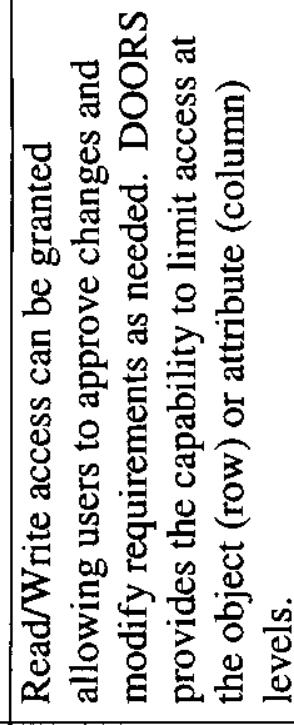 & 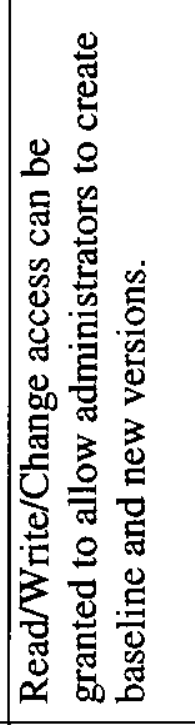 \\
\hline 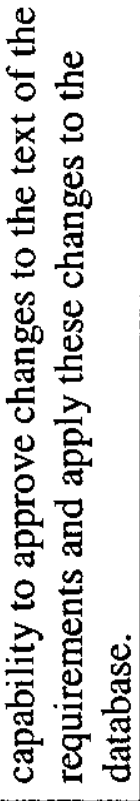 & 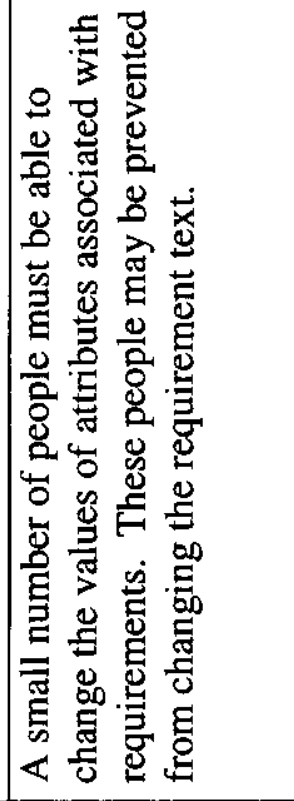 & 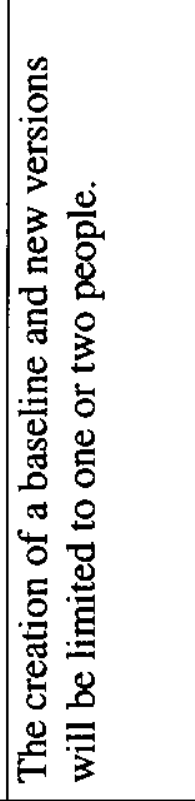 \\
\hline \multirow[t]{2}{*}{ 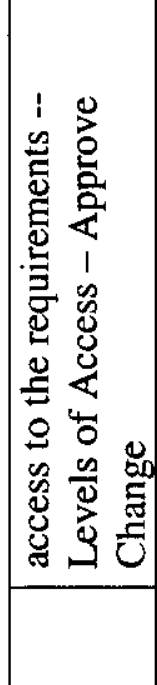 } & 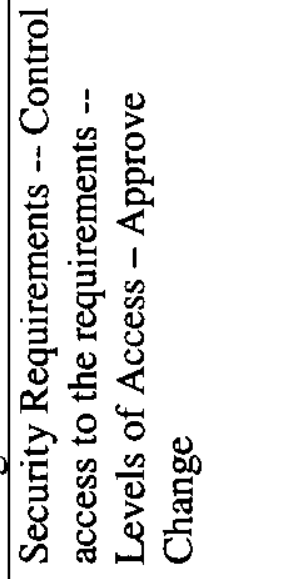 & 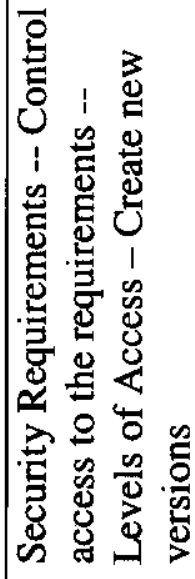 \\
\hline & 이 & $=$ \\
\hline
\end{tabular}

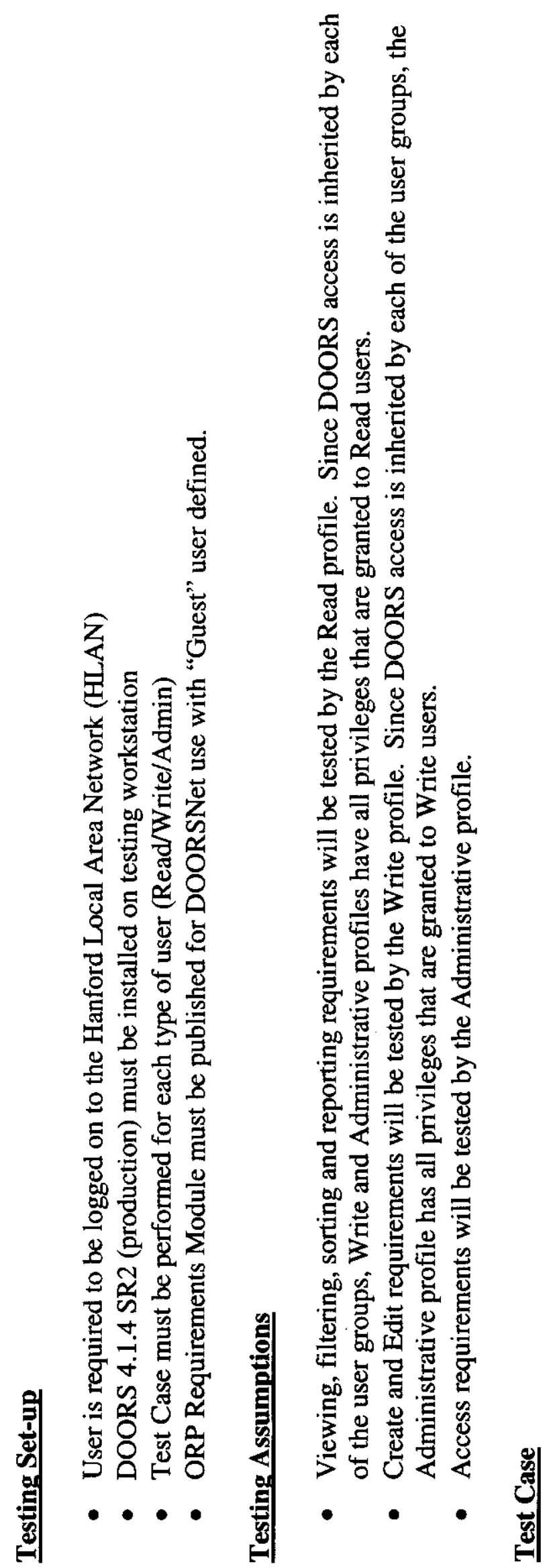




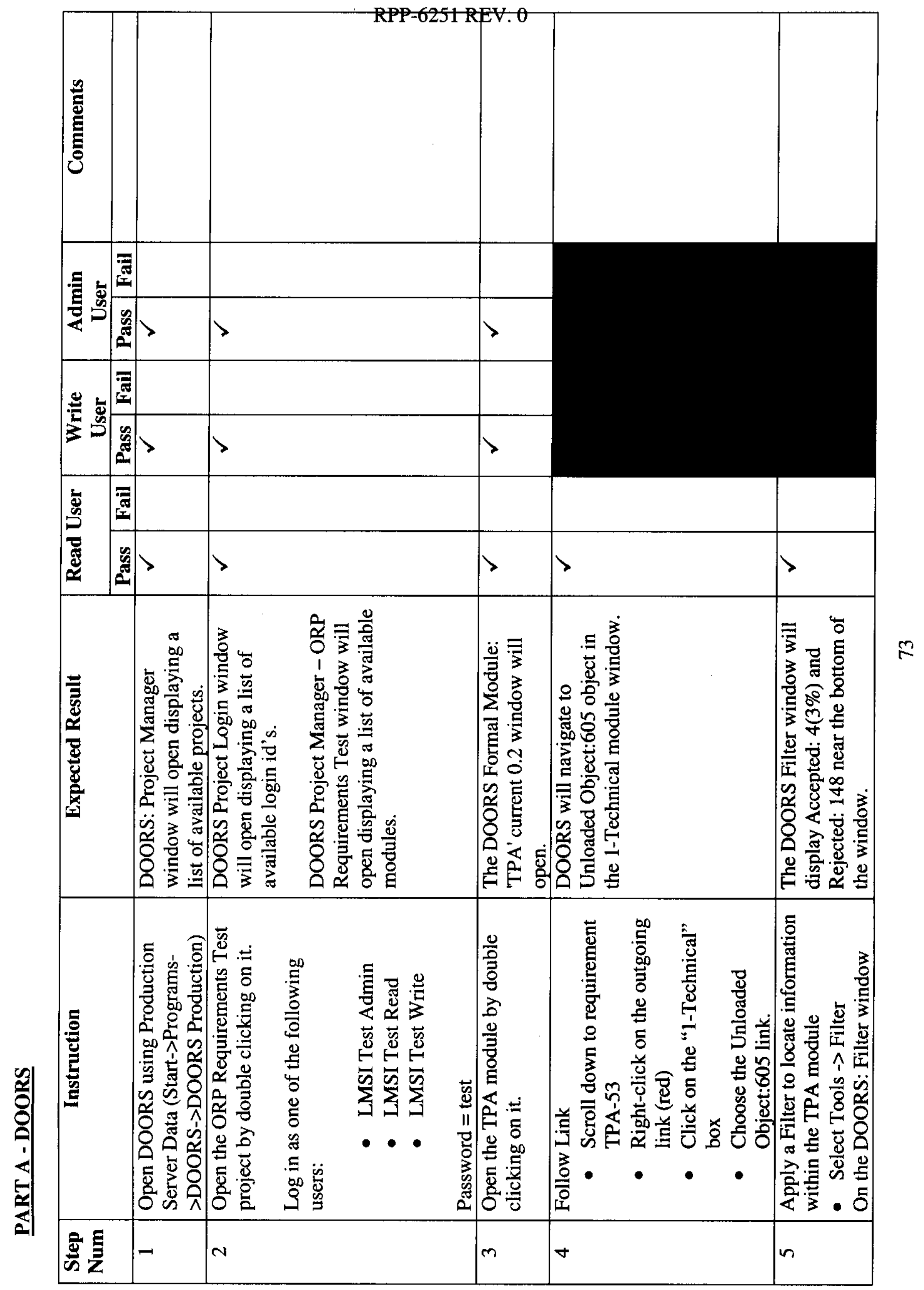




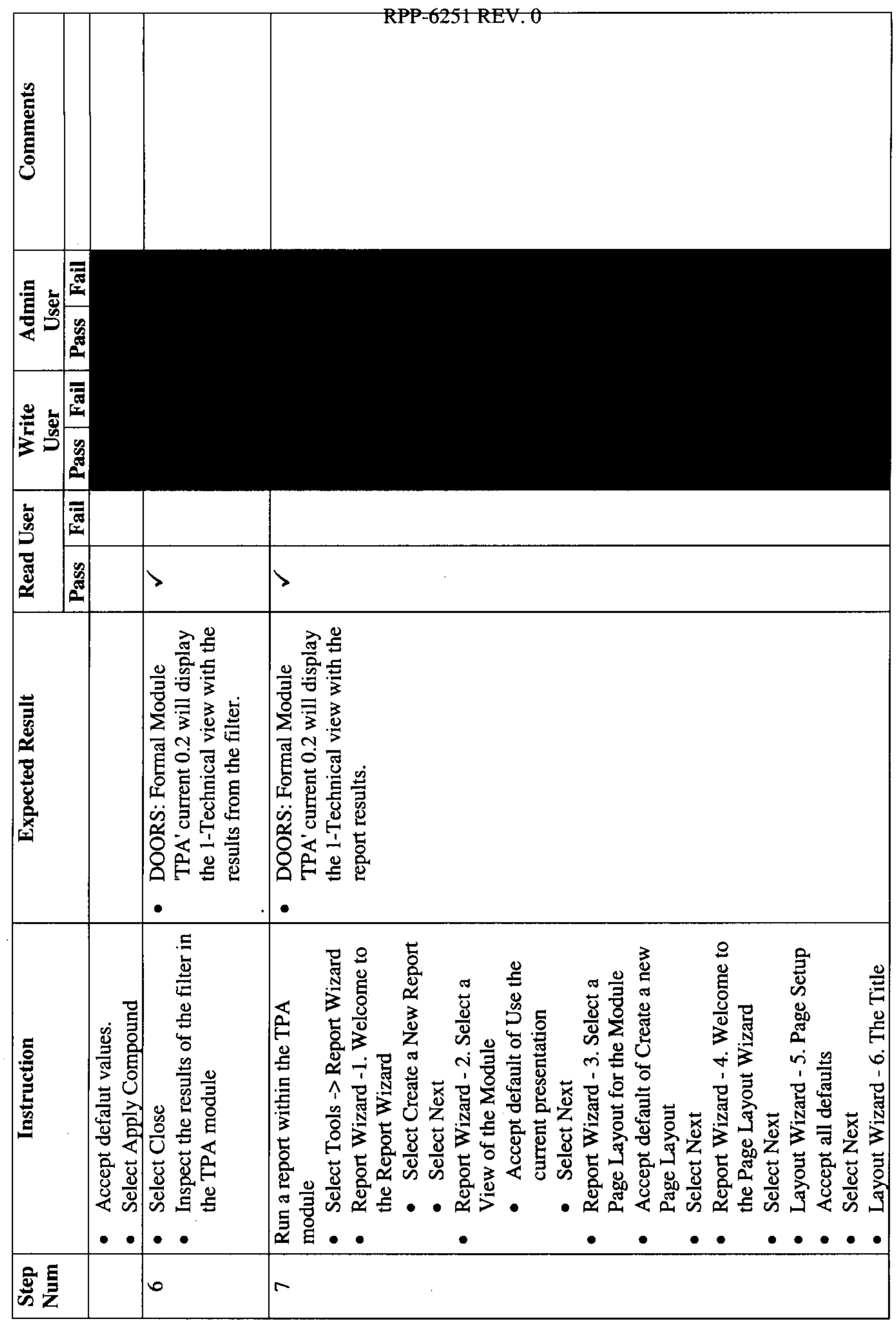




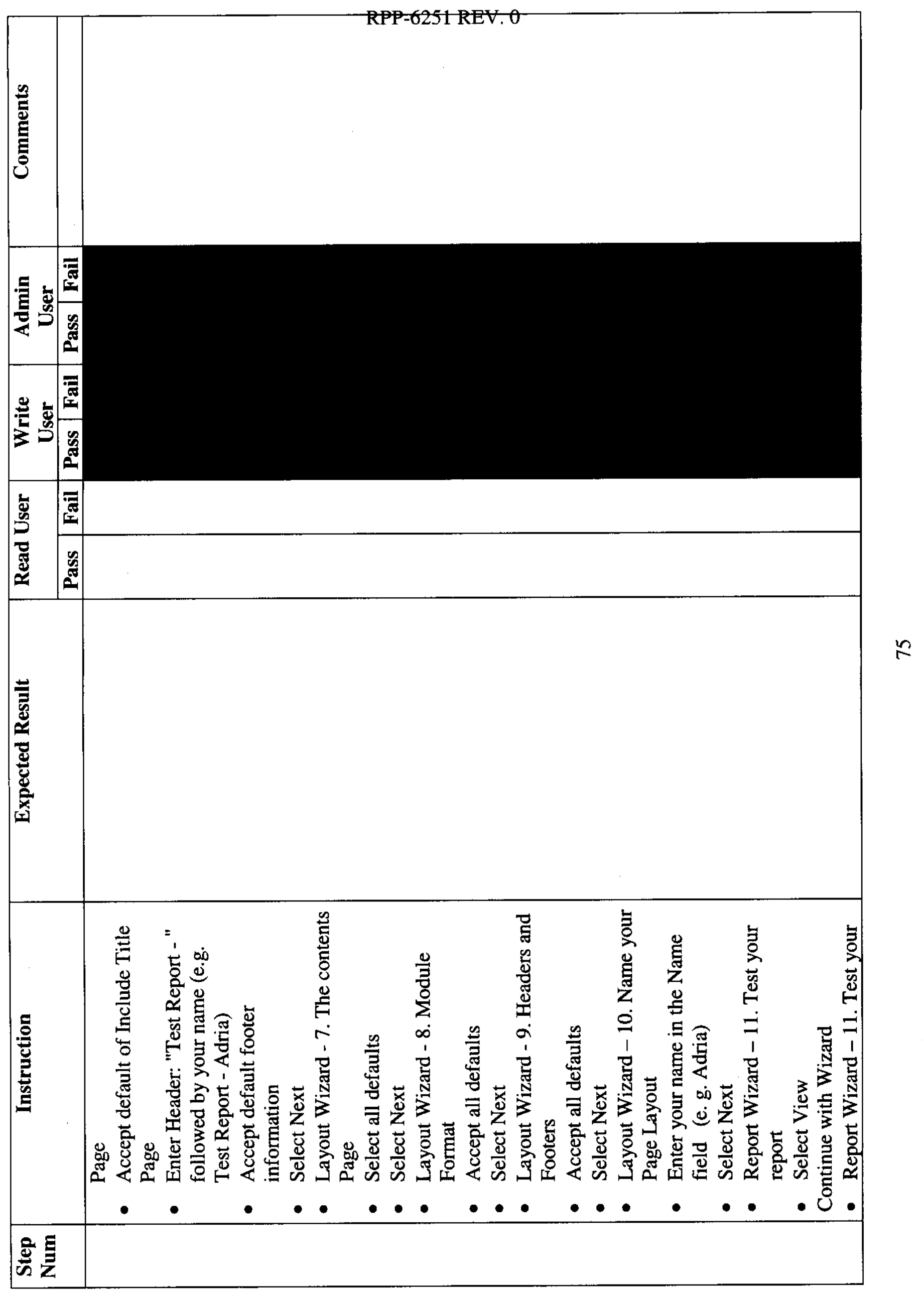




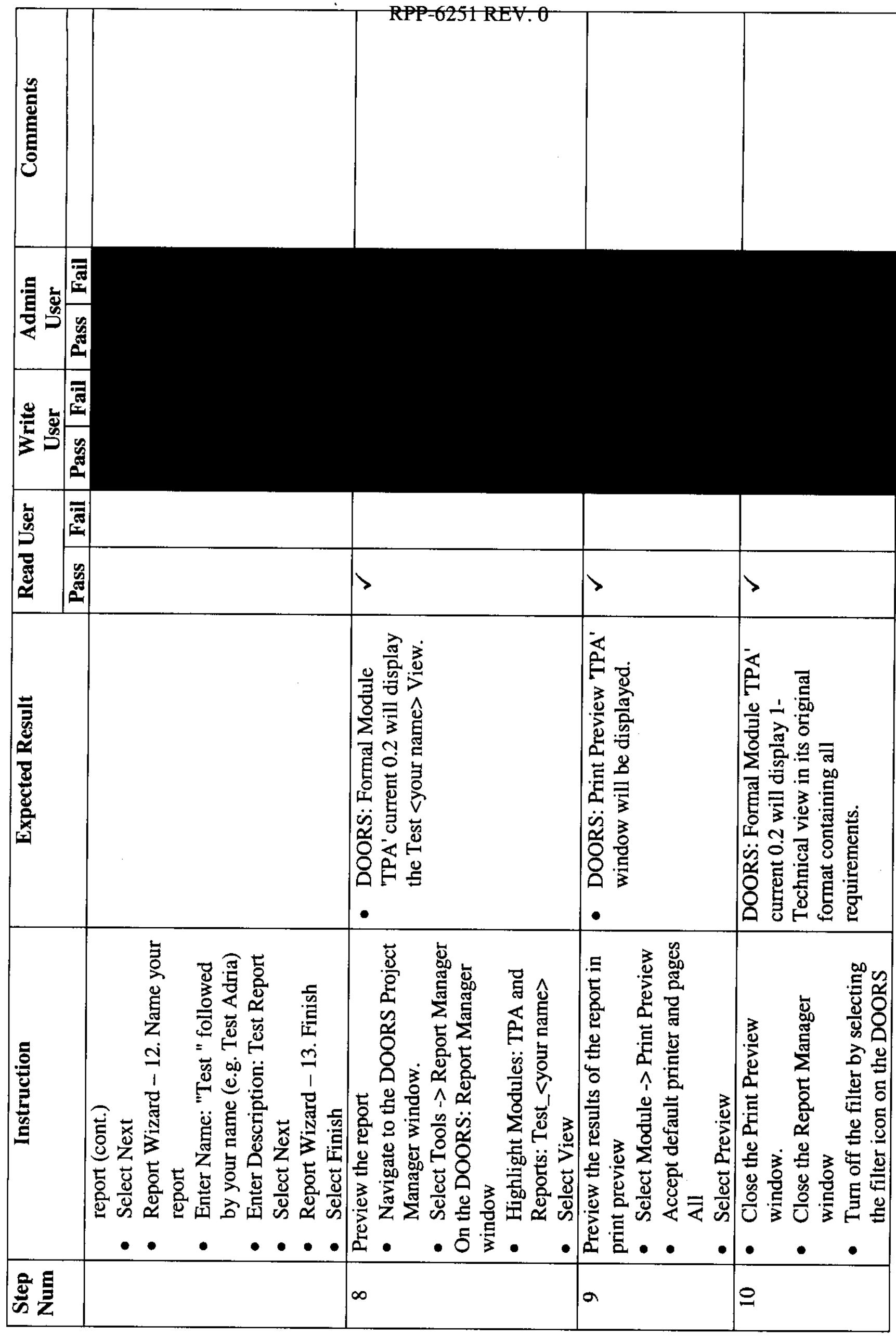




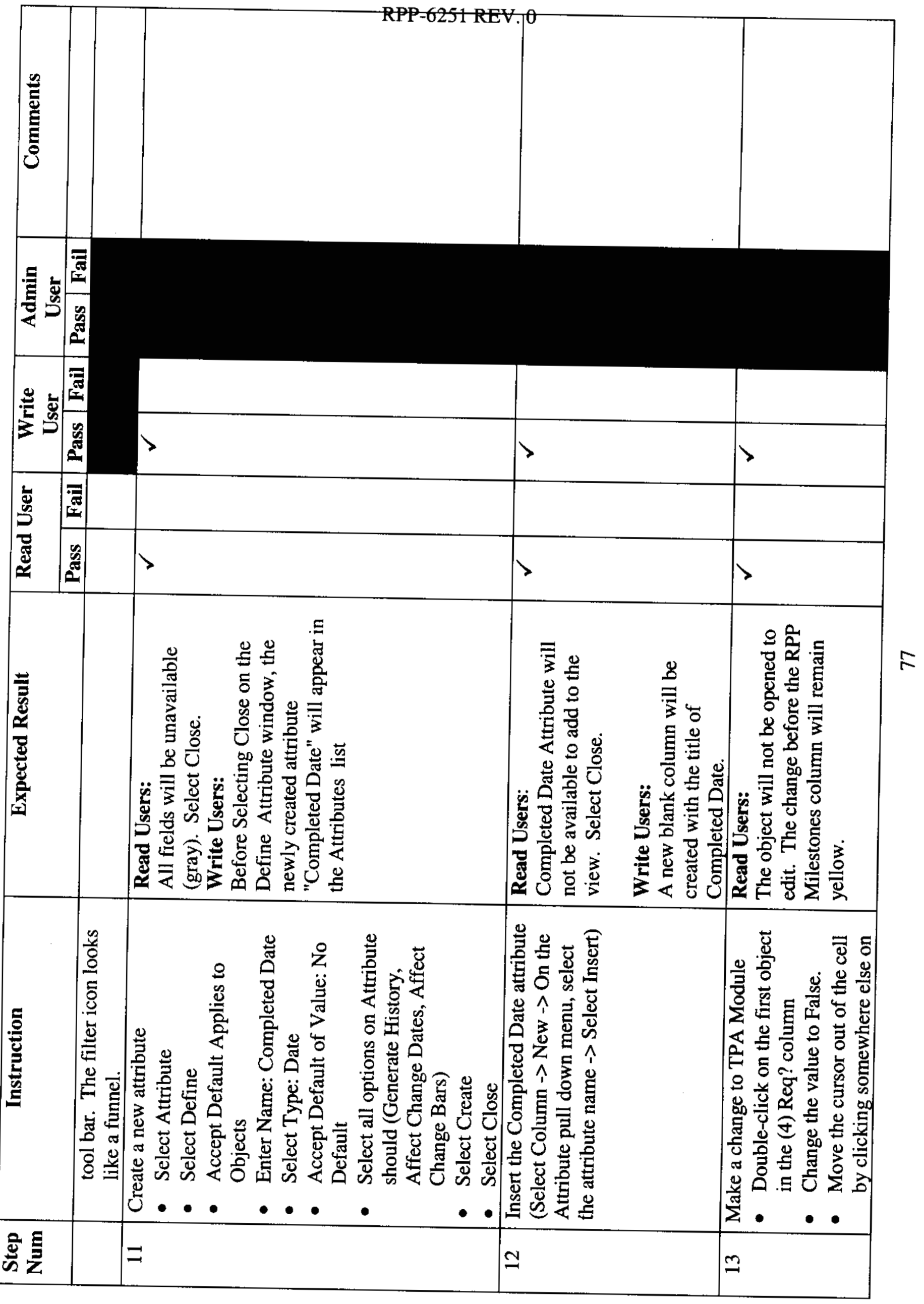




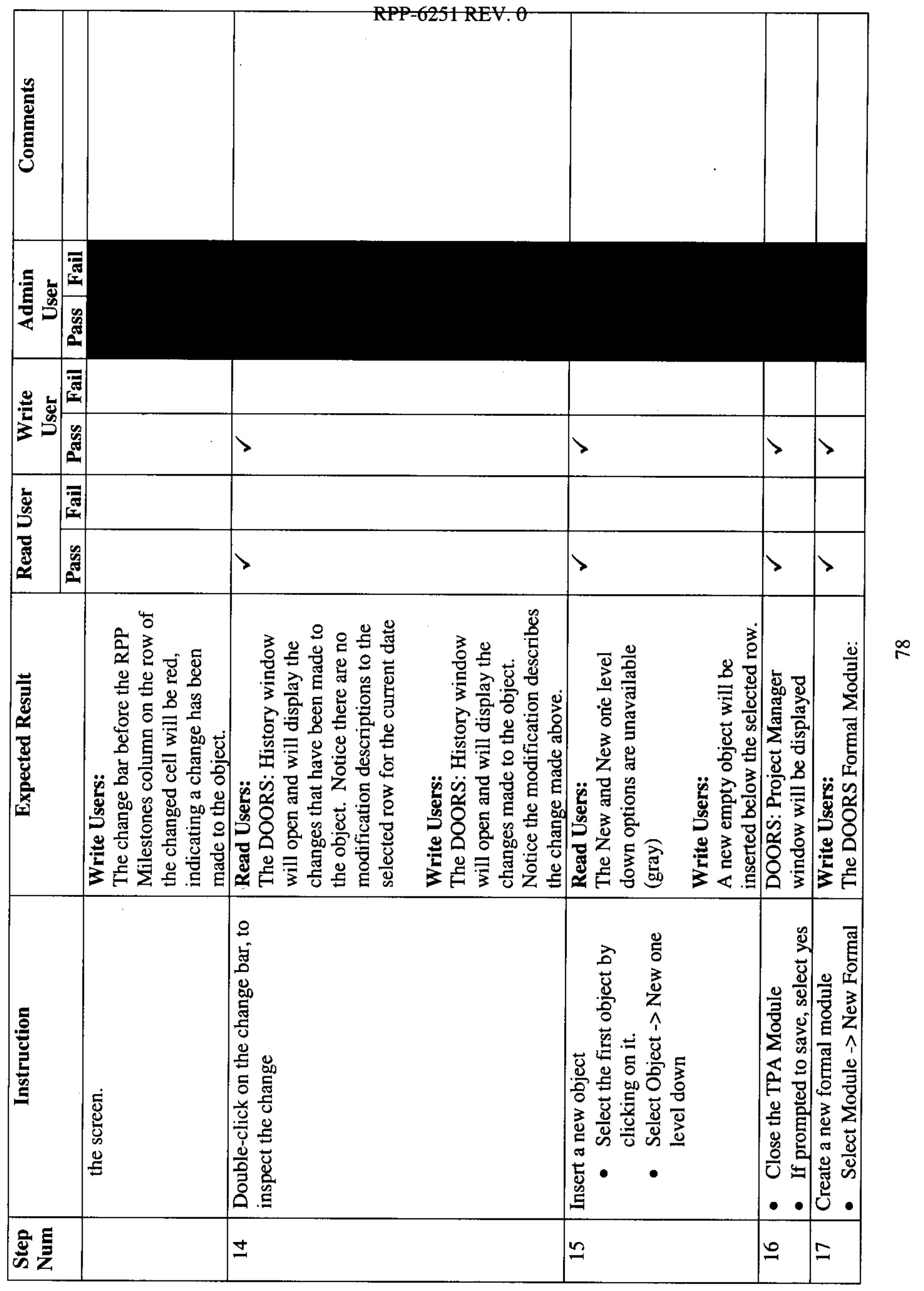




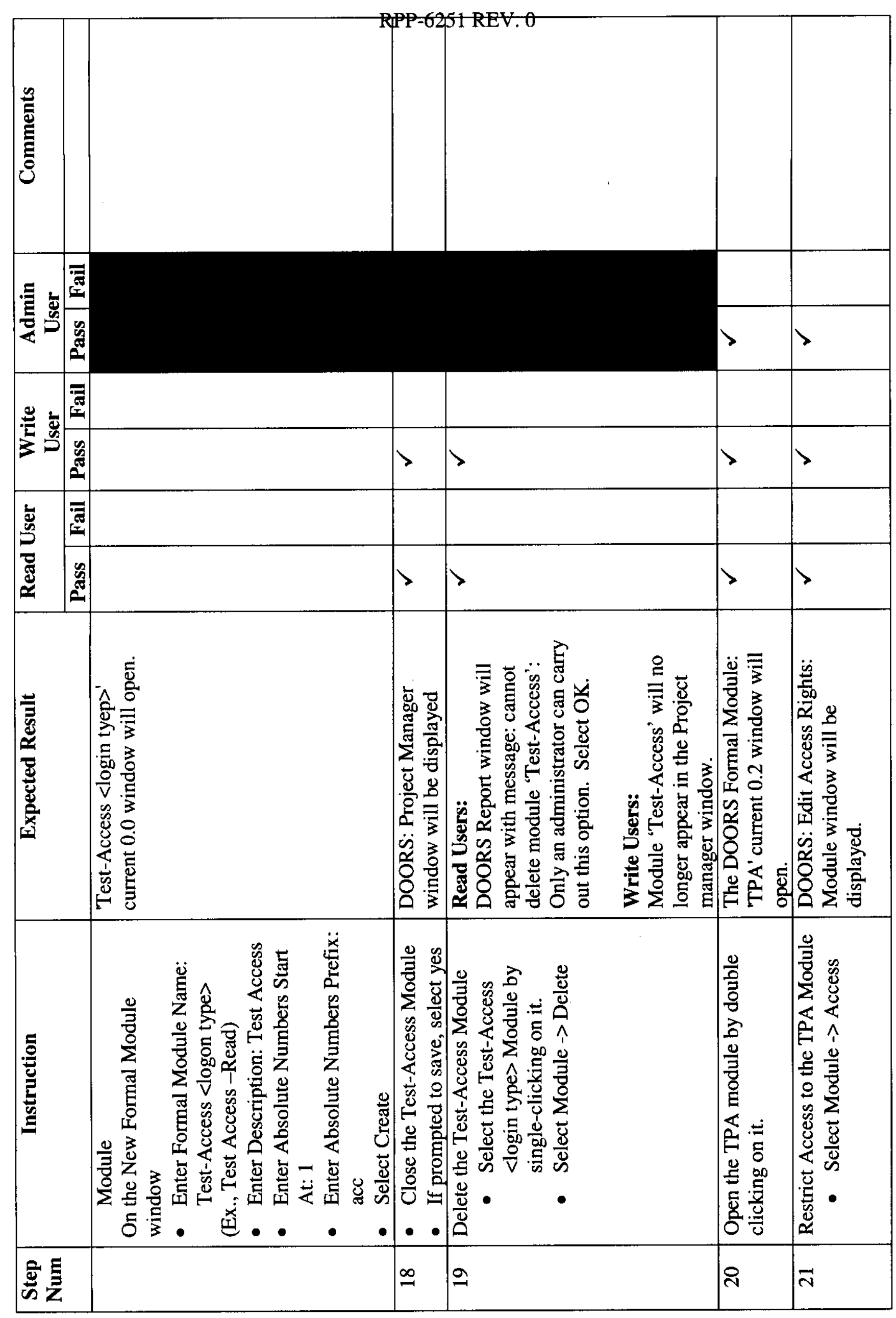




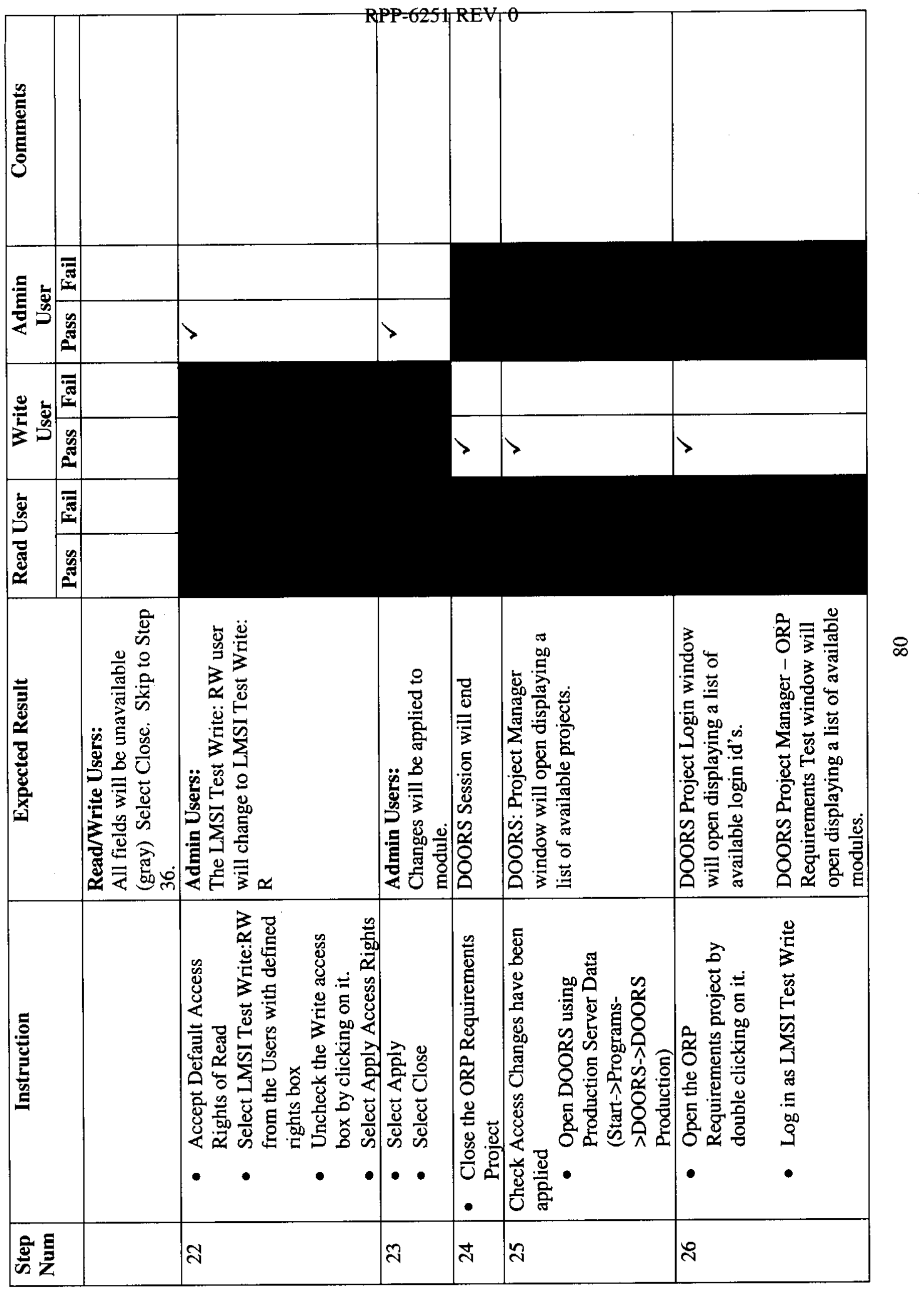




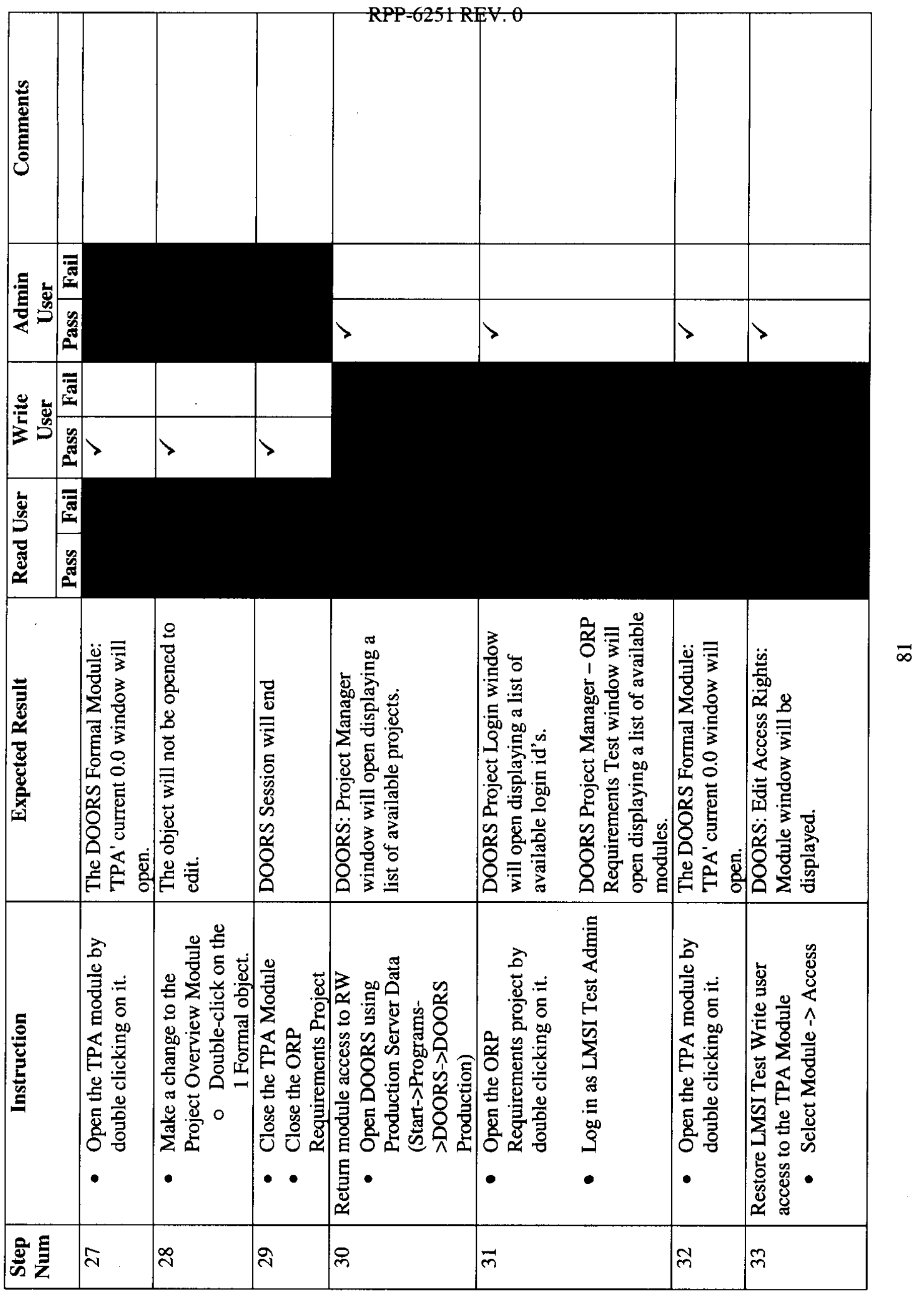




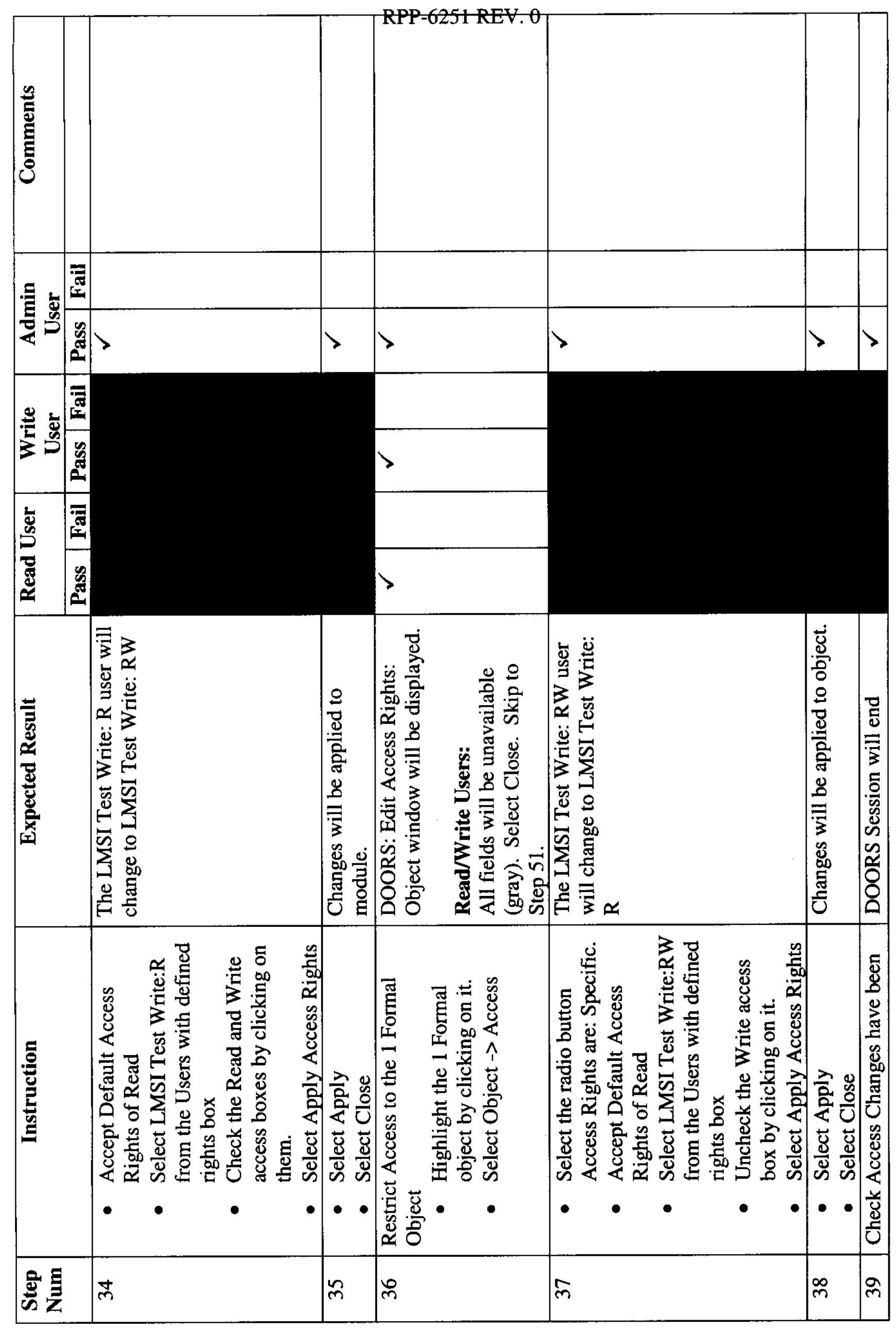




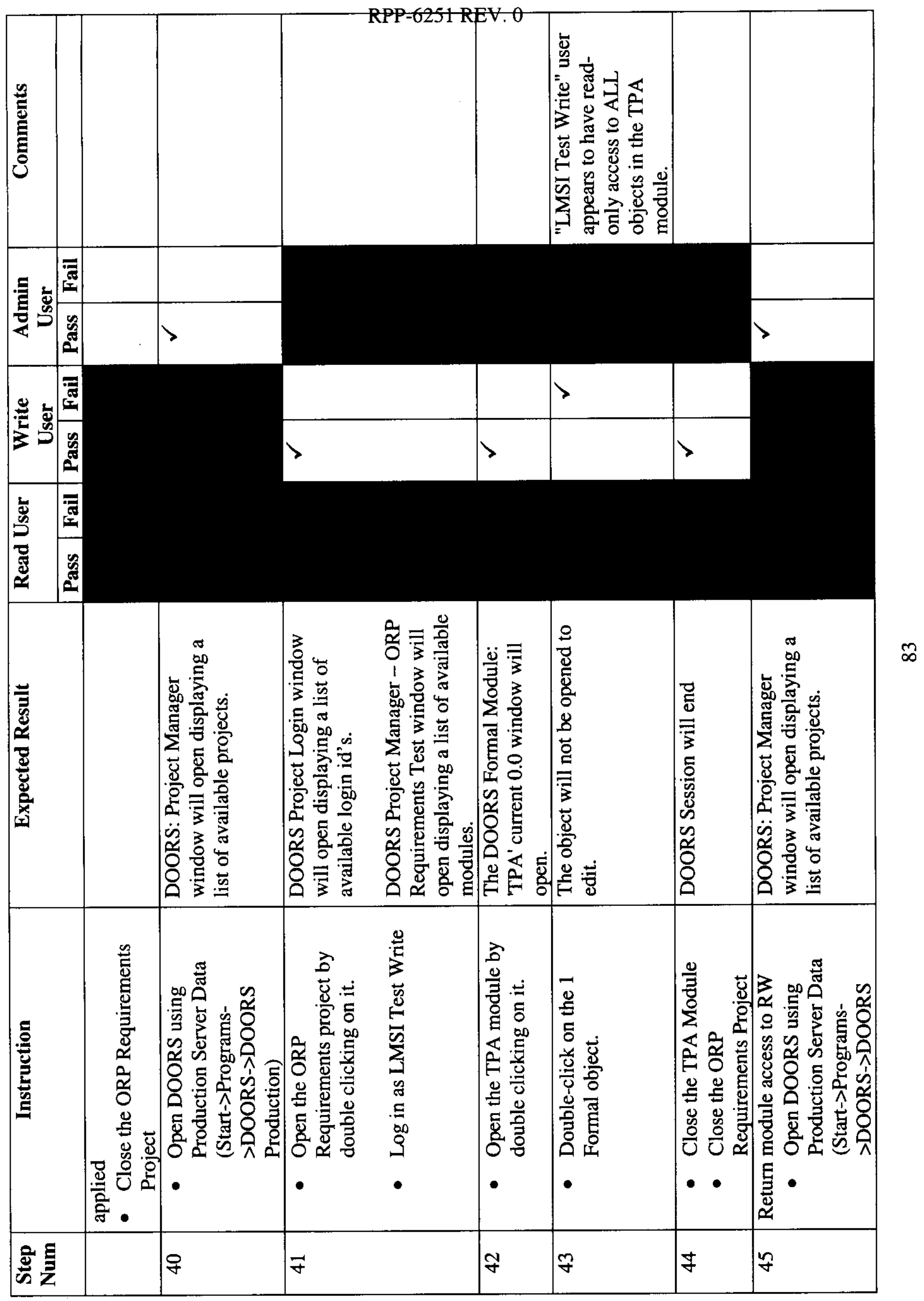




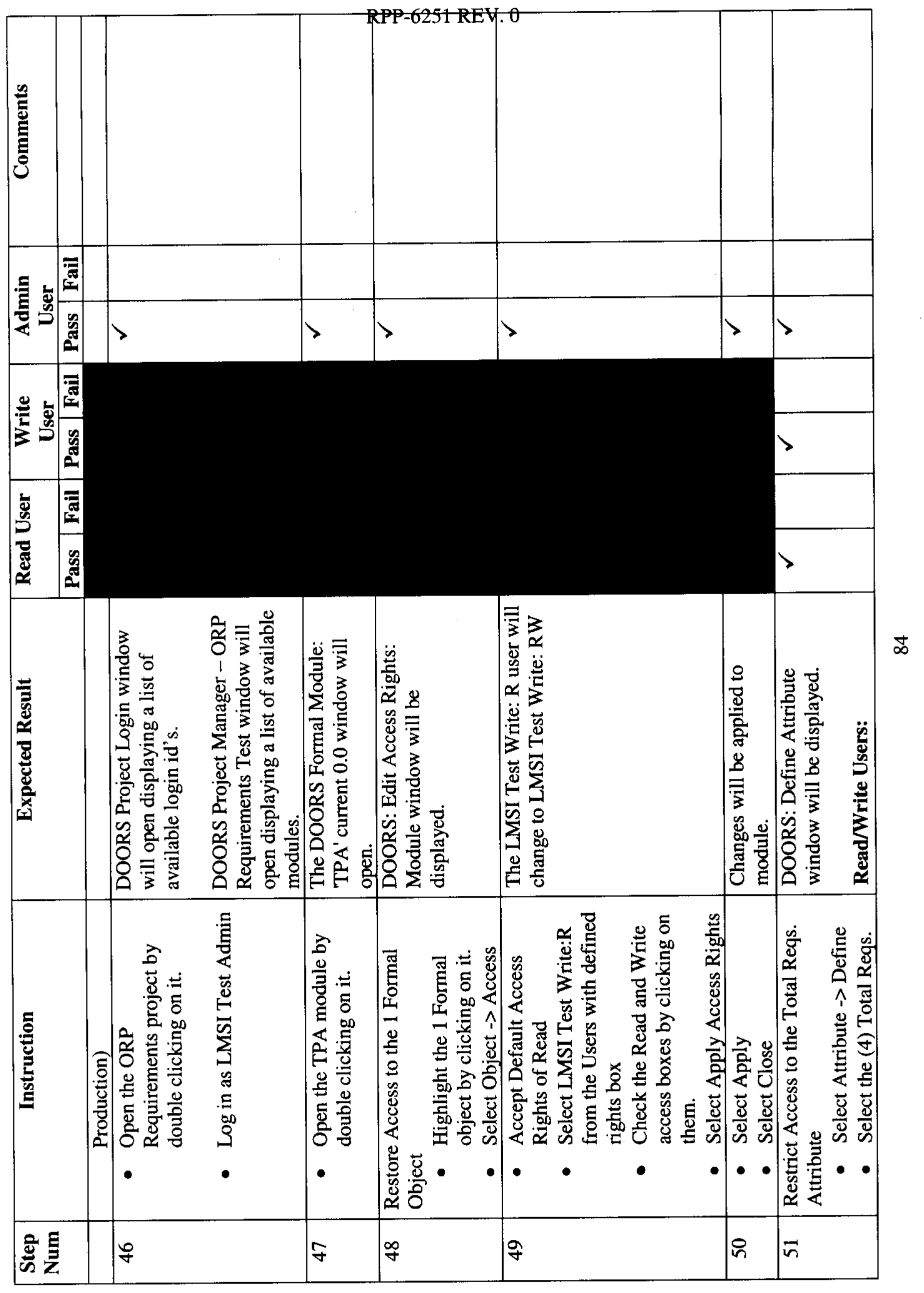




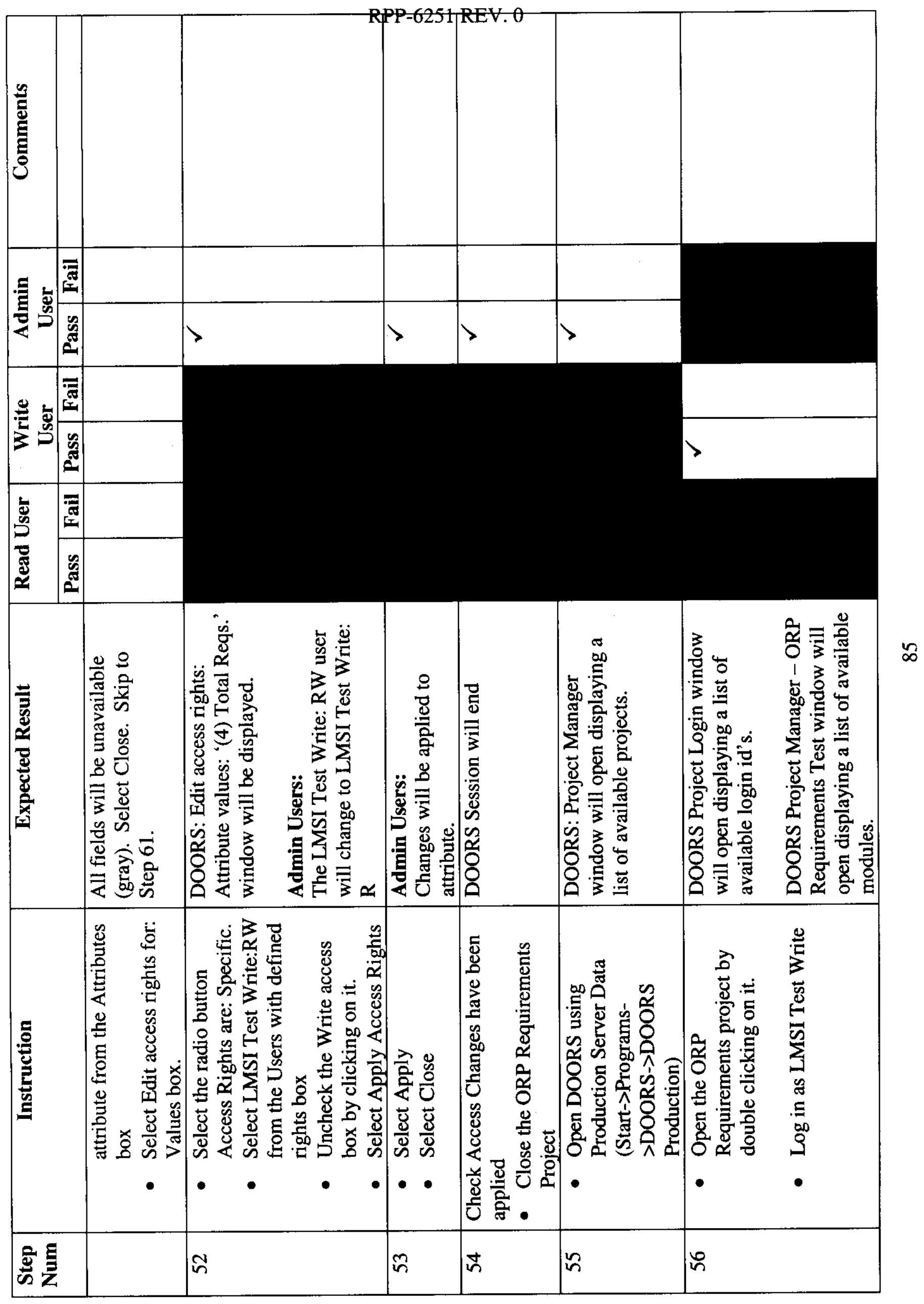




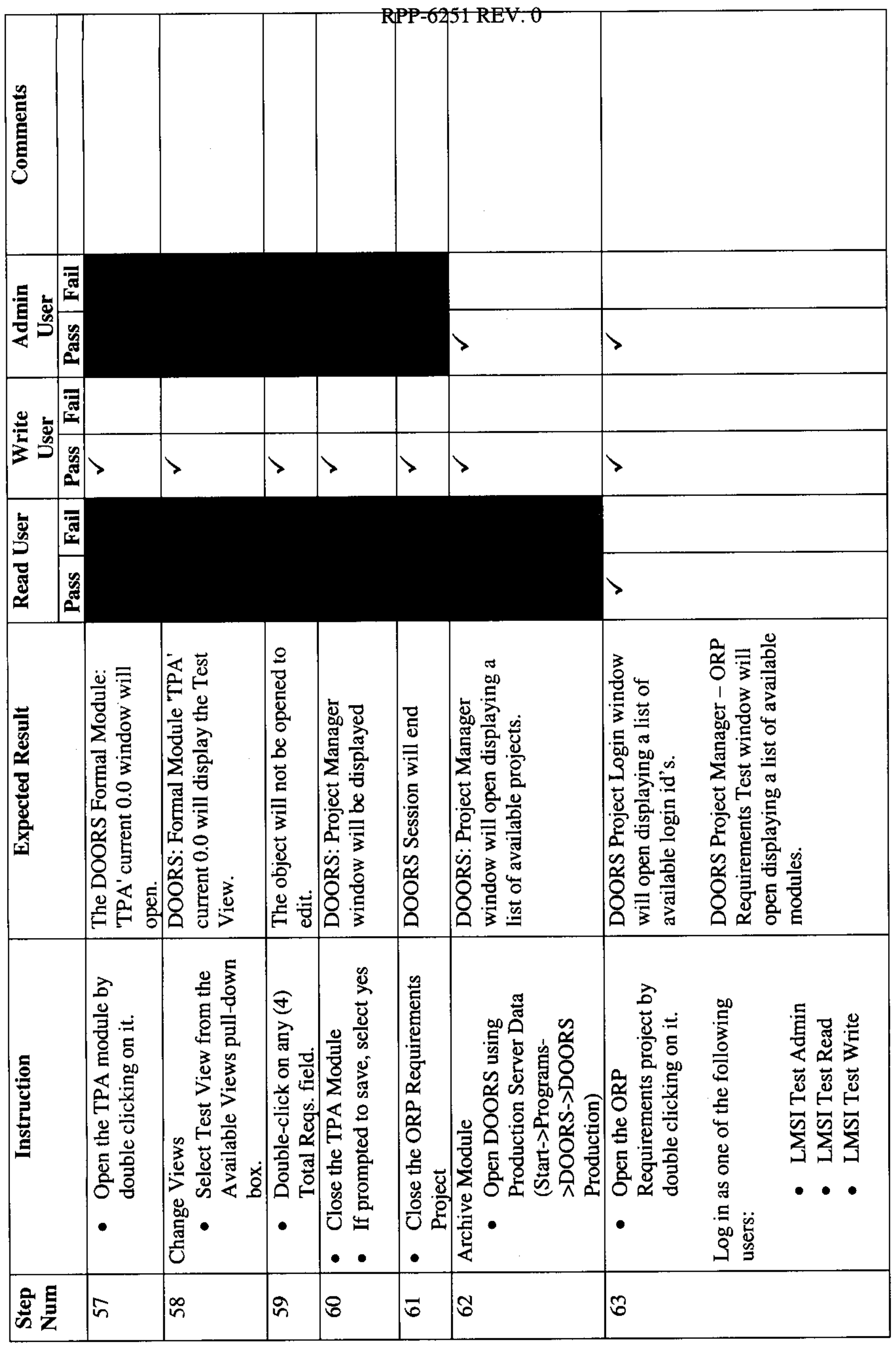




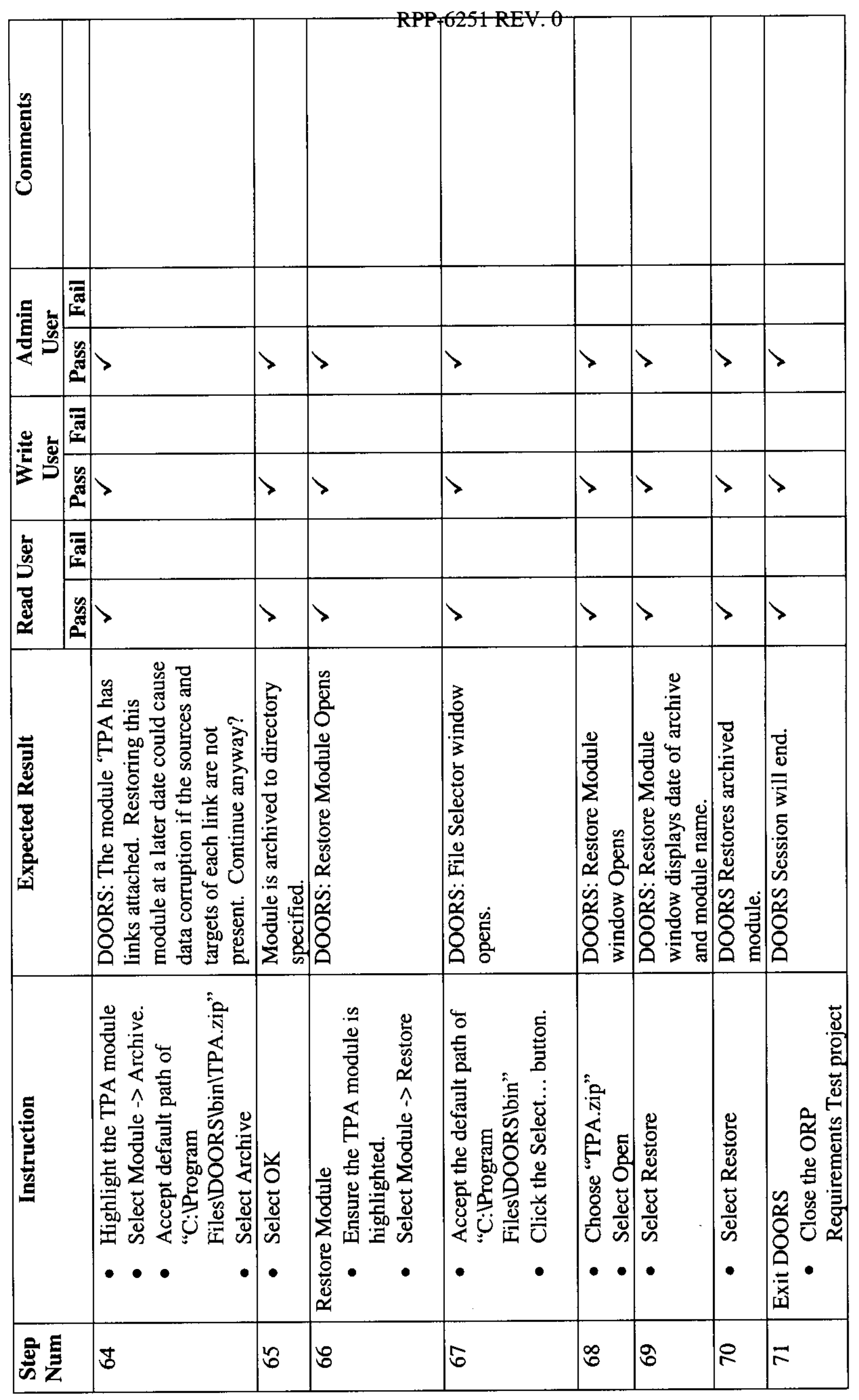




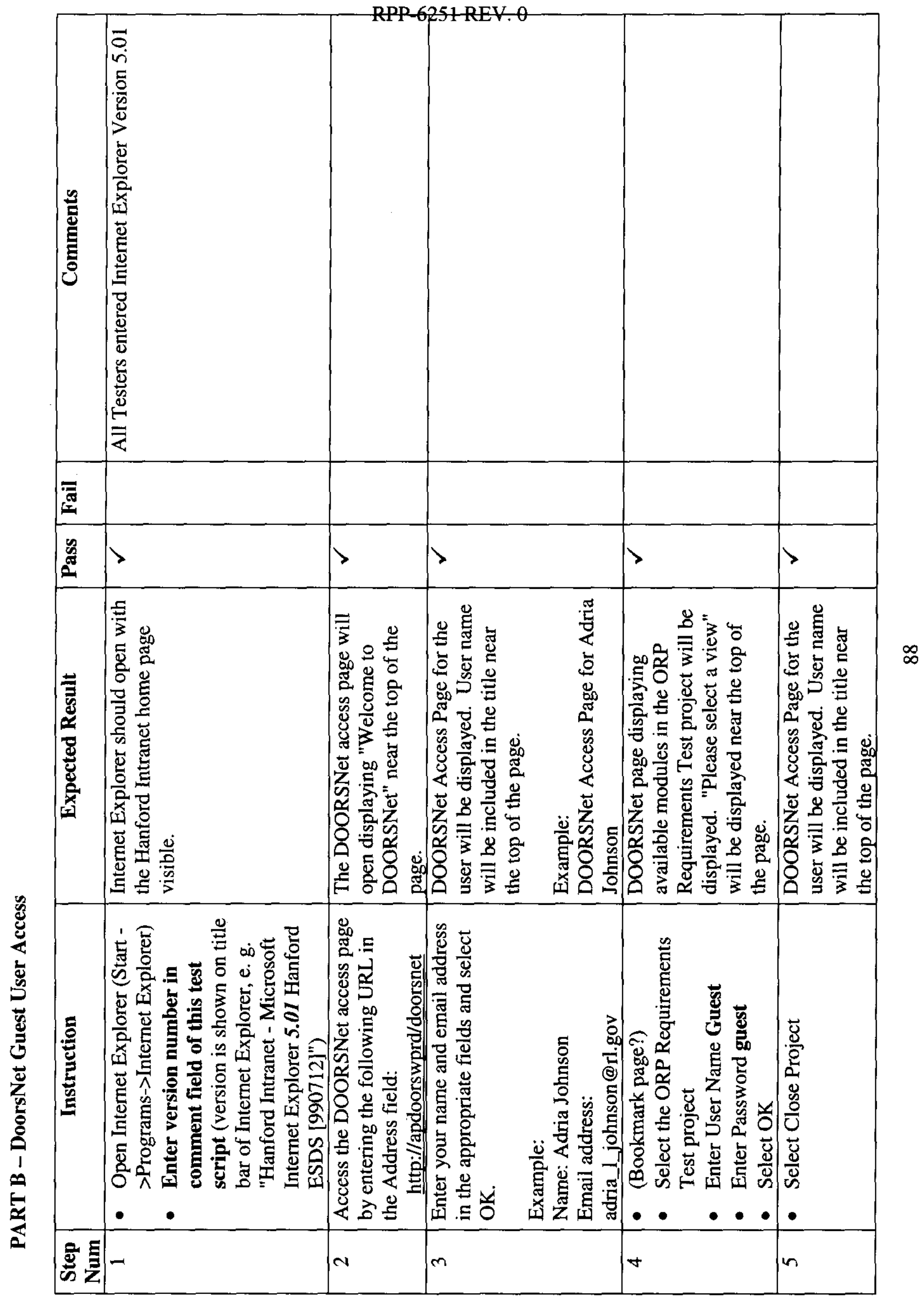




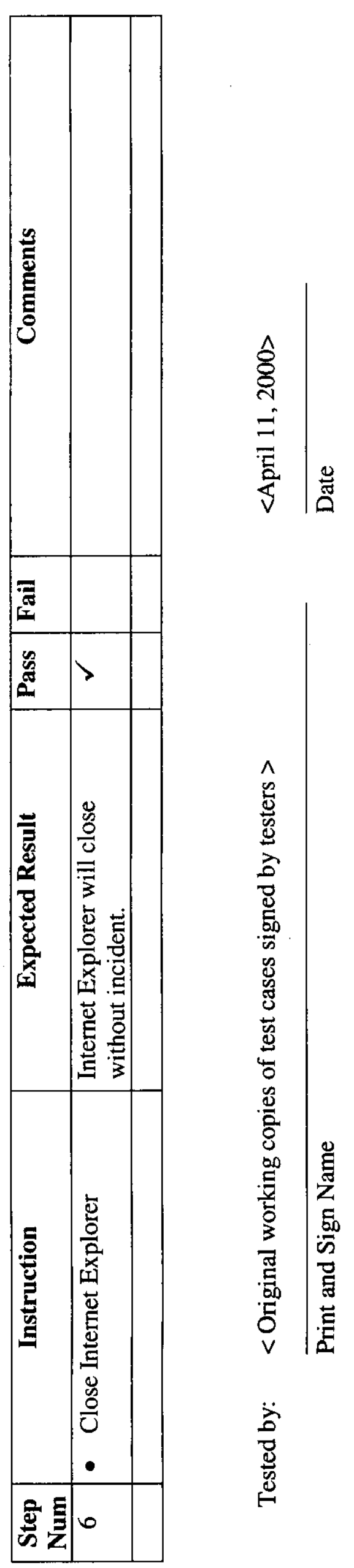

RPP-6251 REV. 0 

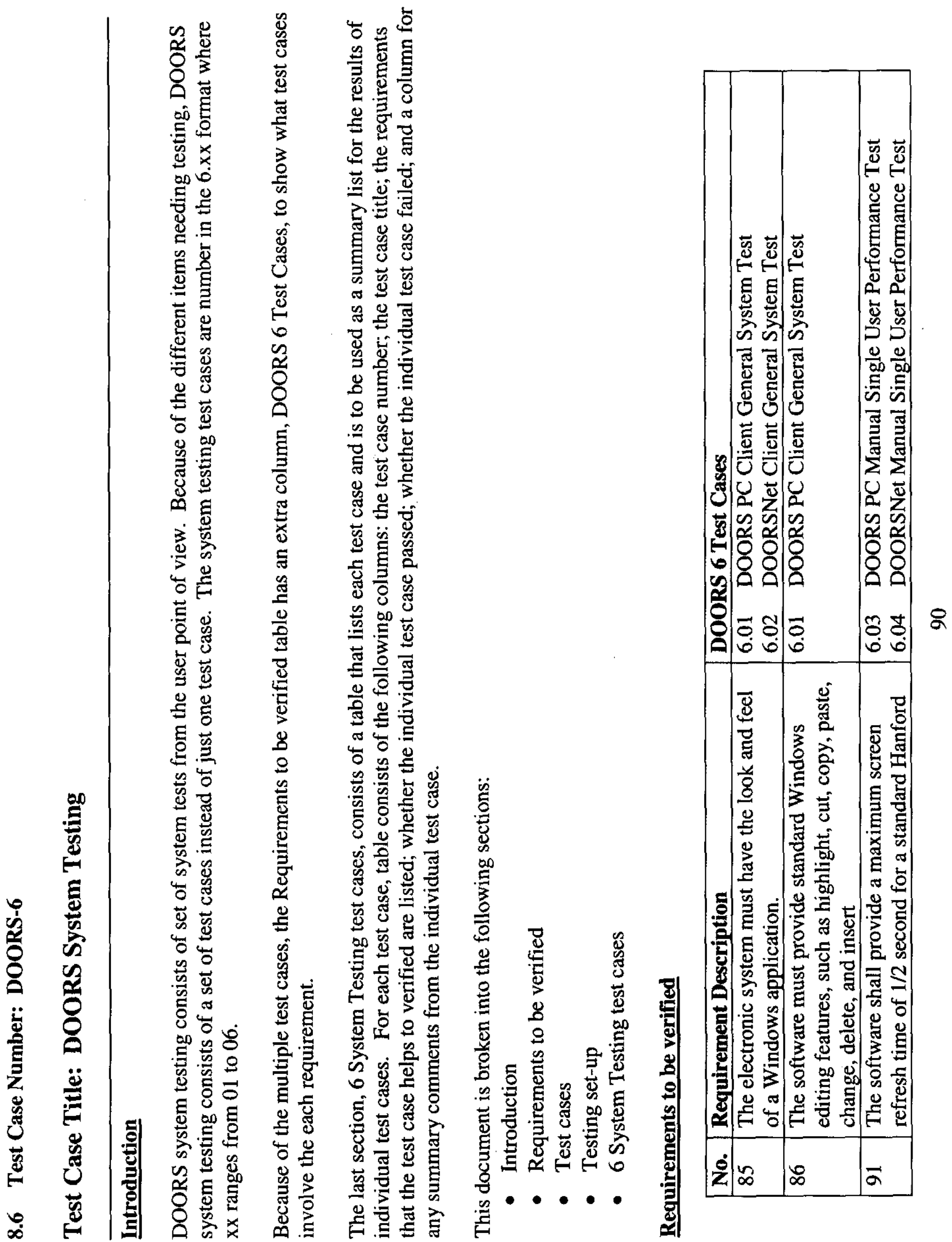
RPP-6251 REV. 0

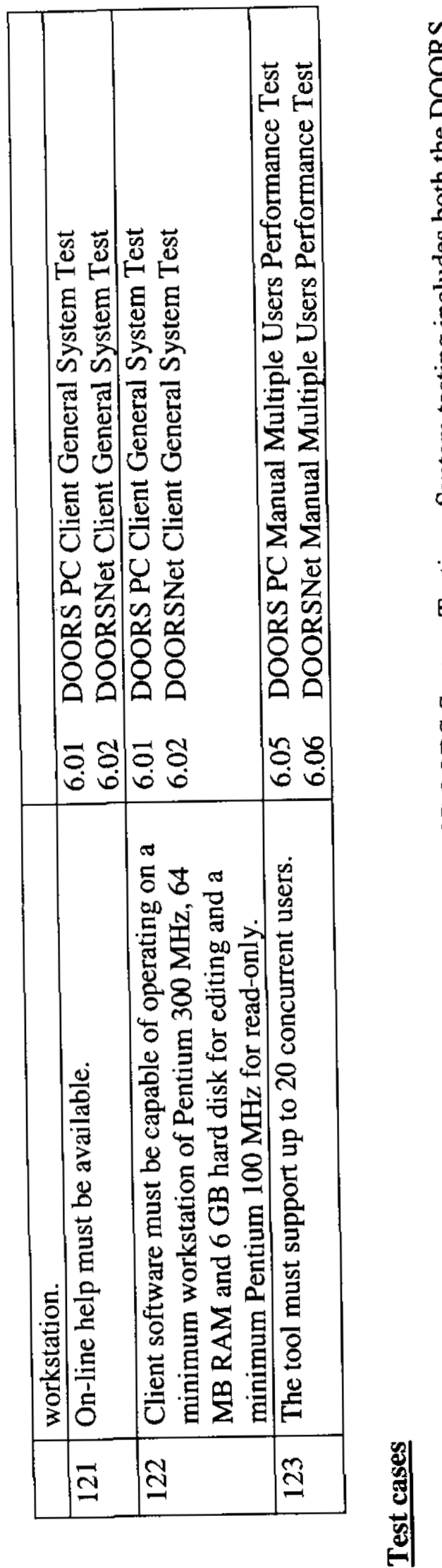

ป

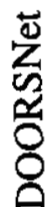

올

로

号棺

总紫玄

$+\stackrel{\overrightarrow{0}}{\mathscr{E}}$

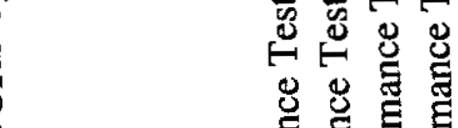

$\rightarrow$ 寻芯

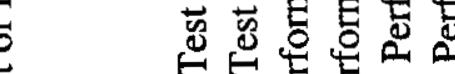

ह

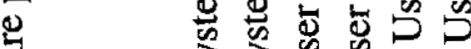

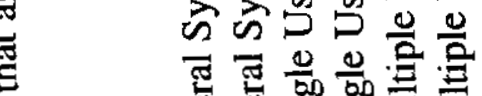

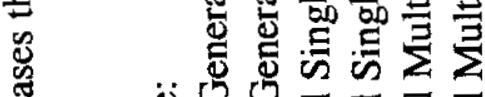

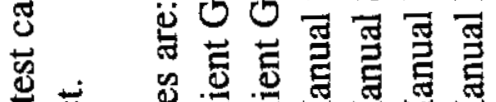

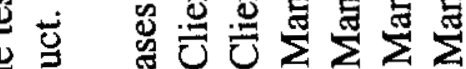

Ð $U \cup U$

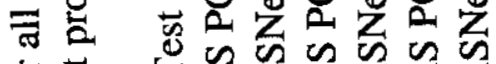

包

壳 至

잉

מ

ำํํㅇํㅇㅎํ

은

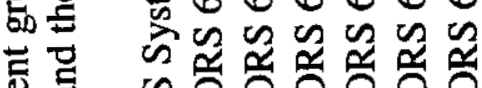

空

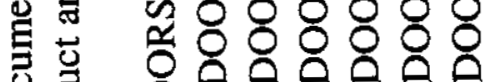

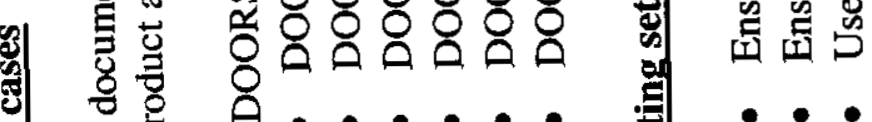

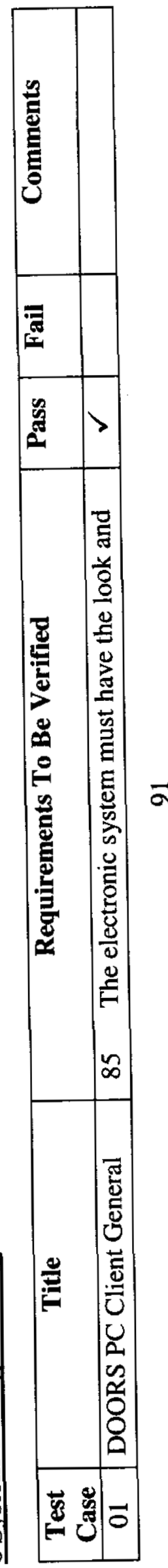


RPP-6251 REV. 0

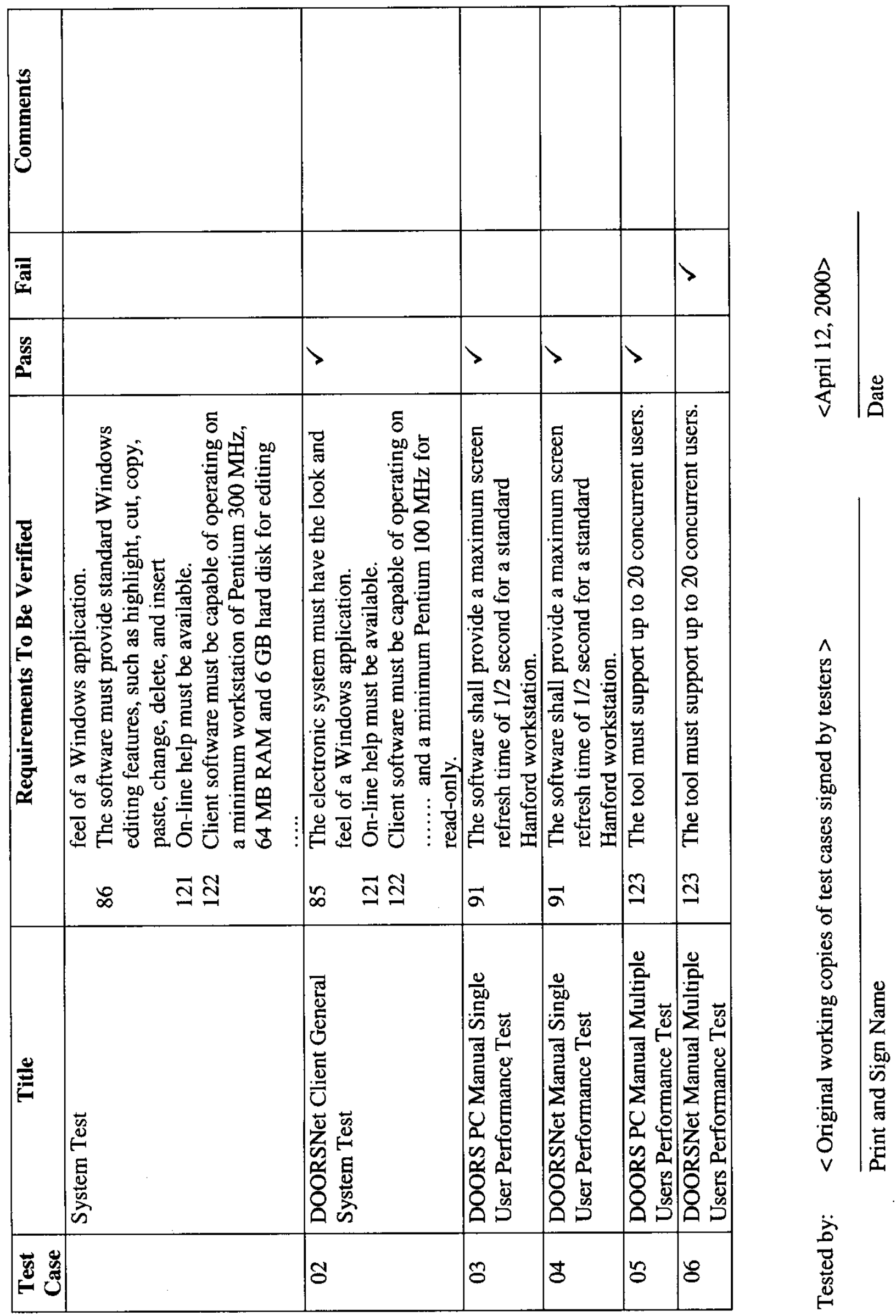



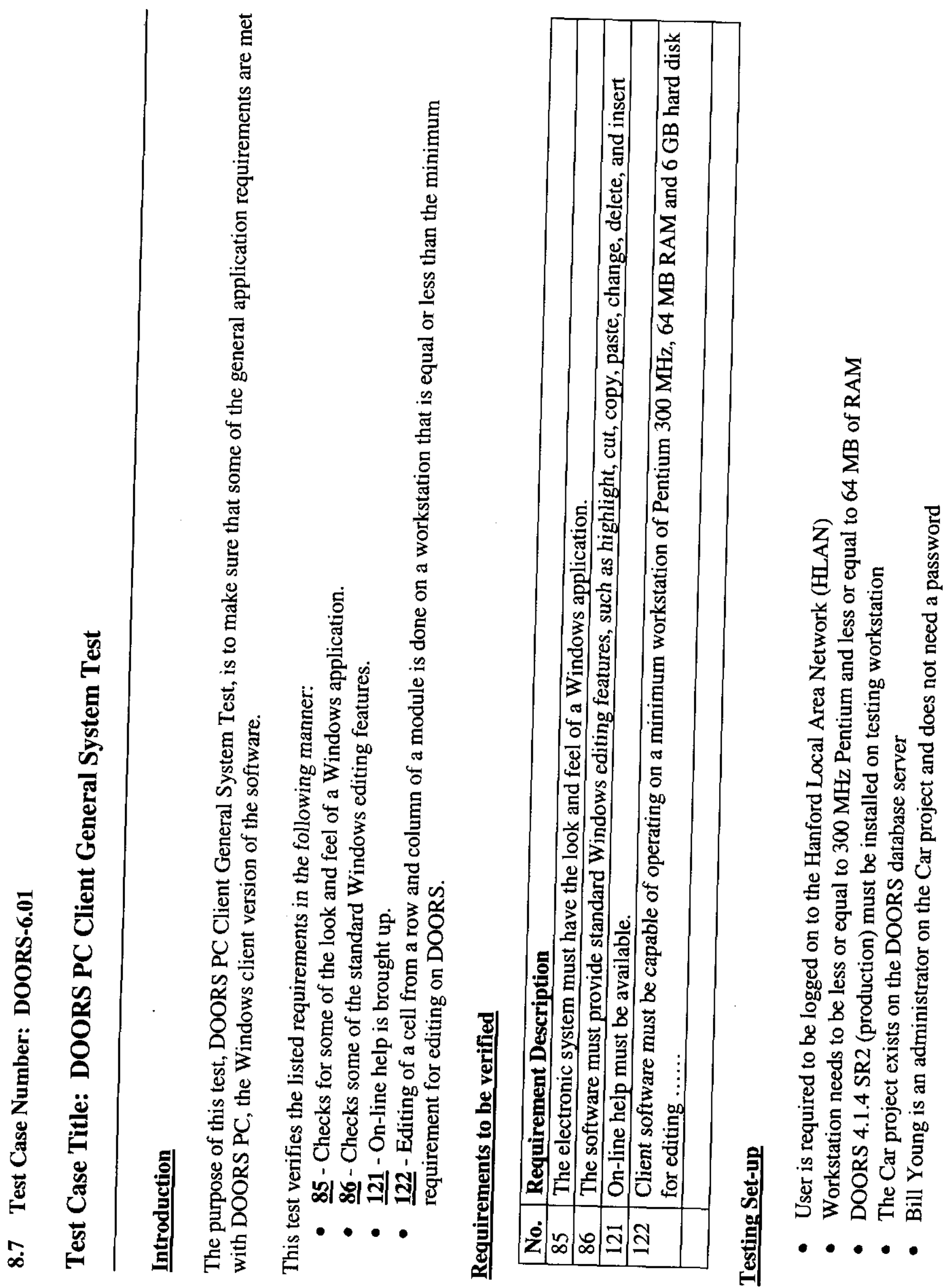
RPP-6251 REV. 0

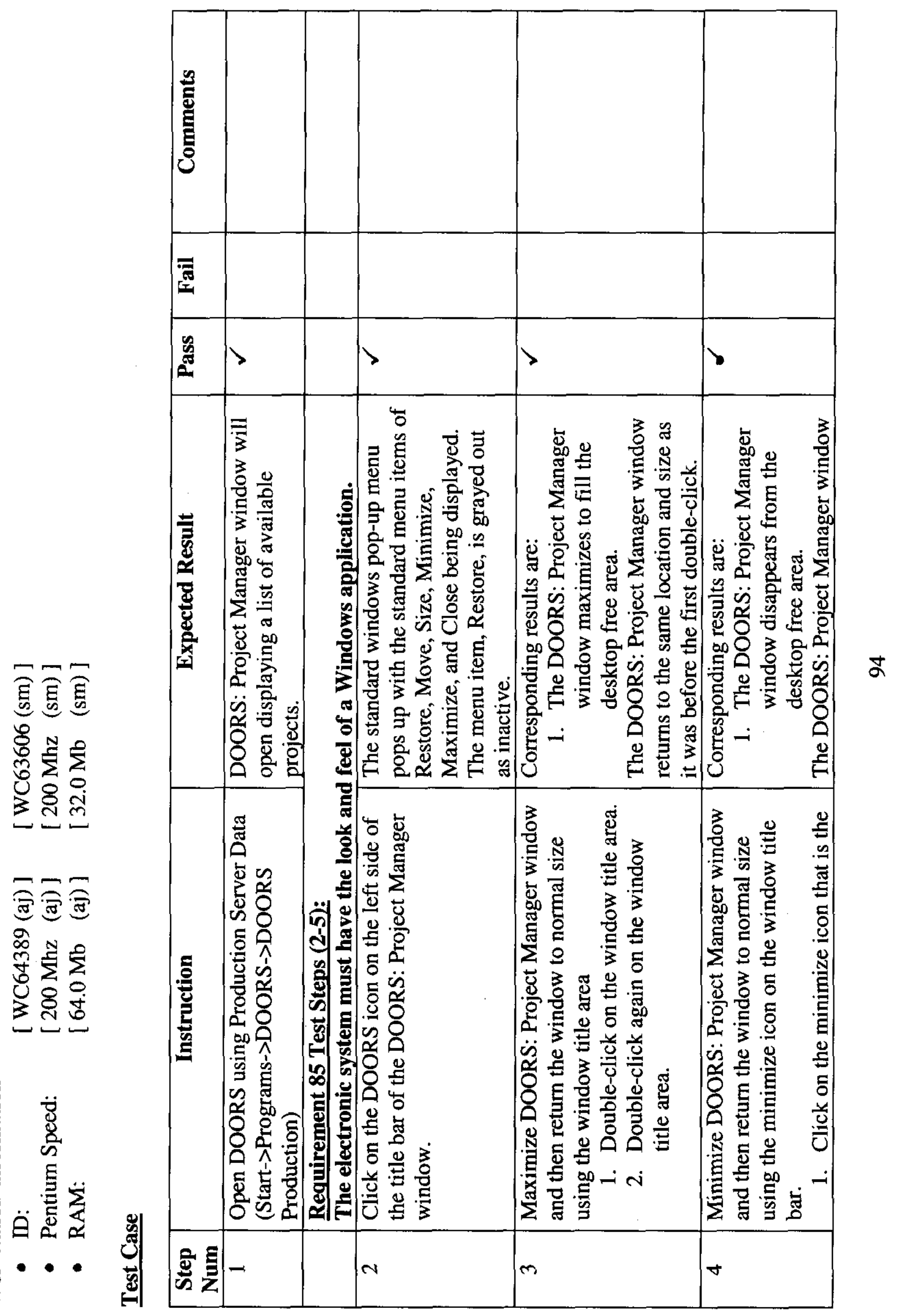




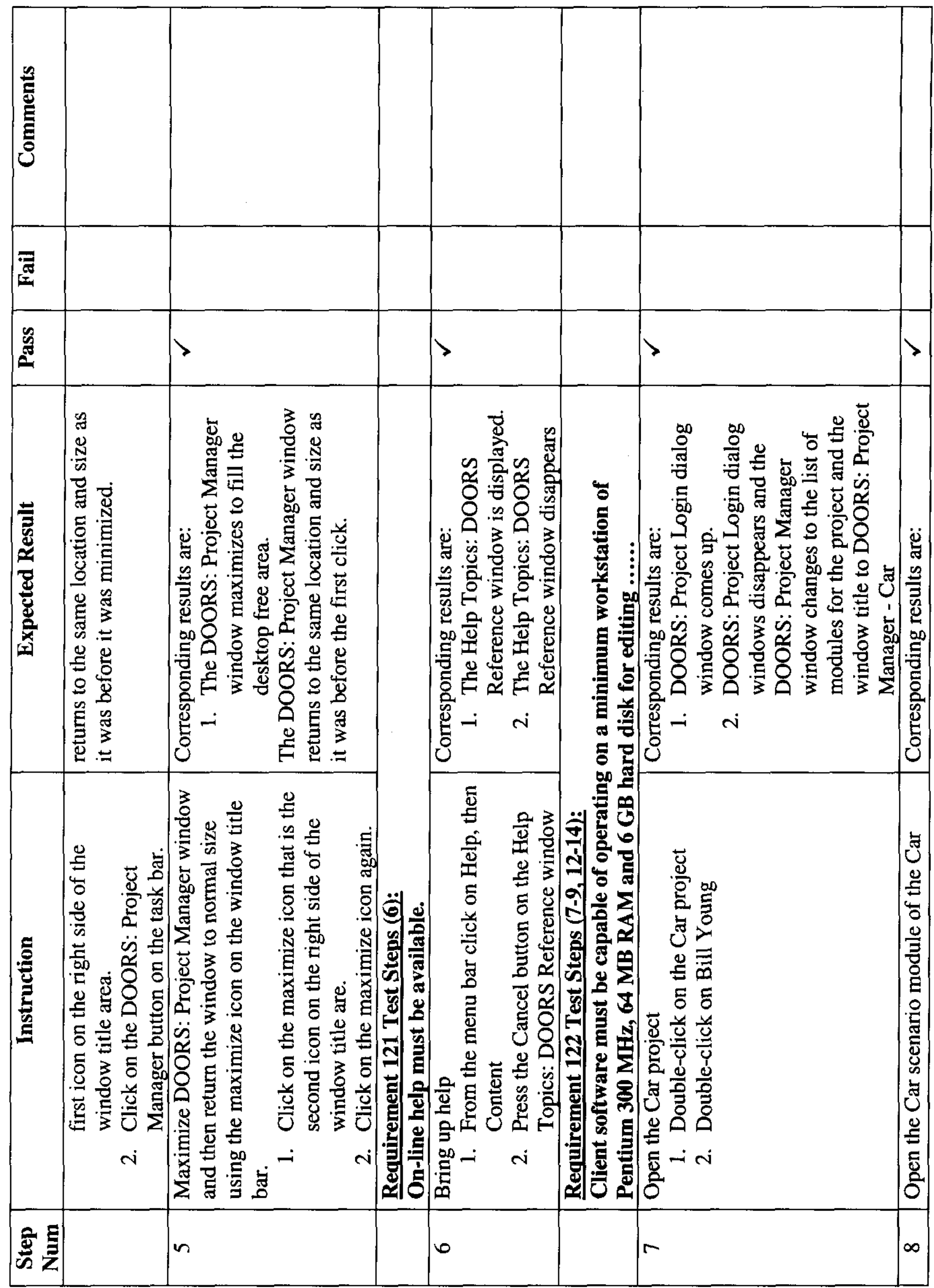


RPP-6251 REV. 0

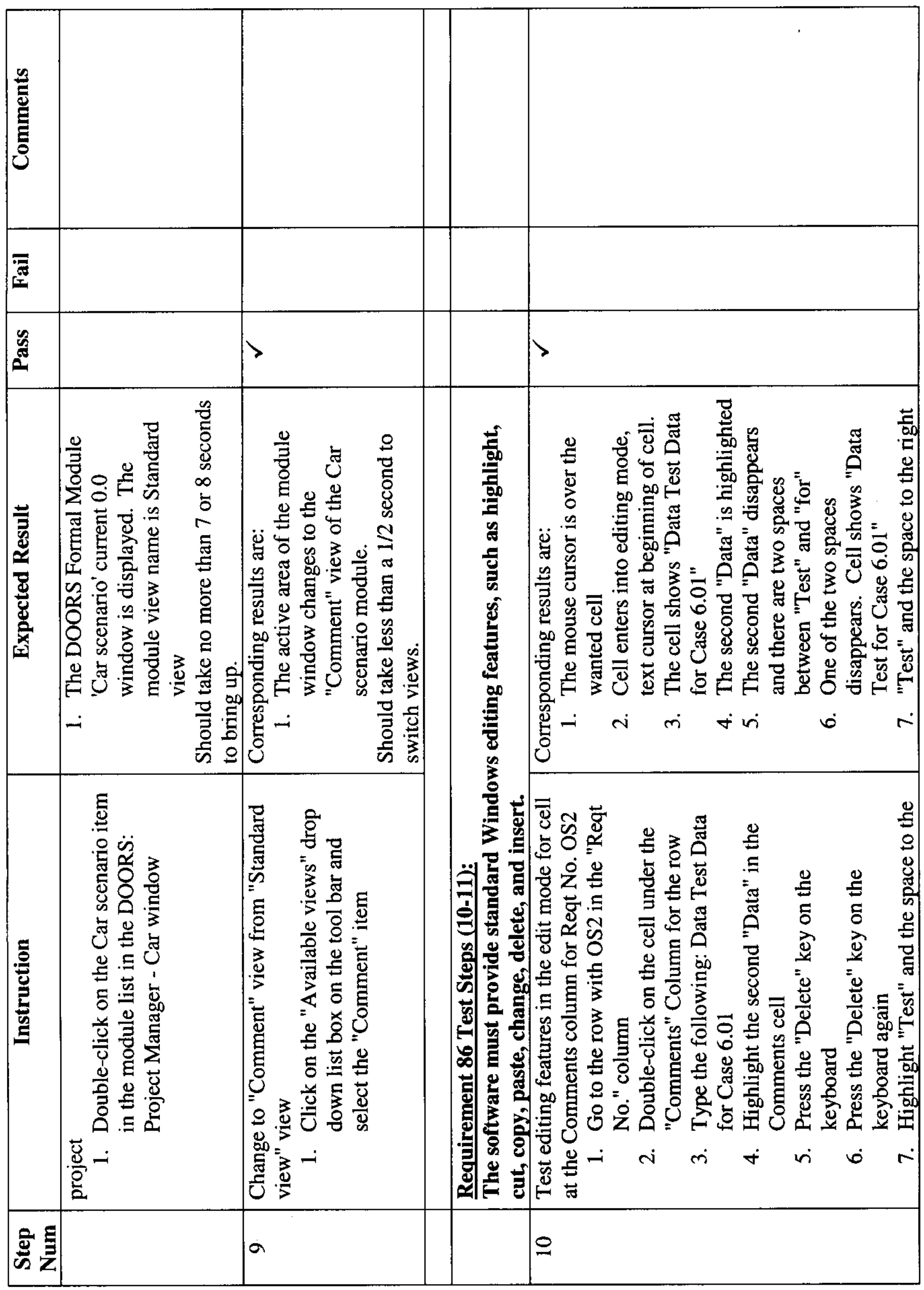


RPP-6251 REV. 0

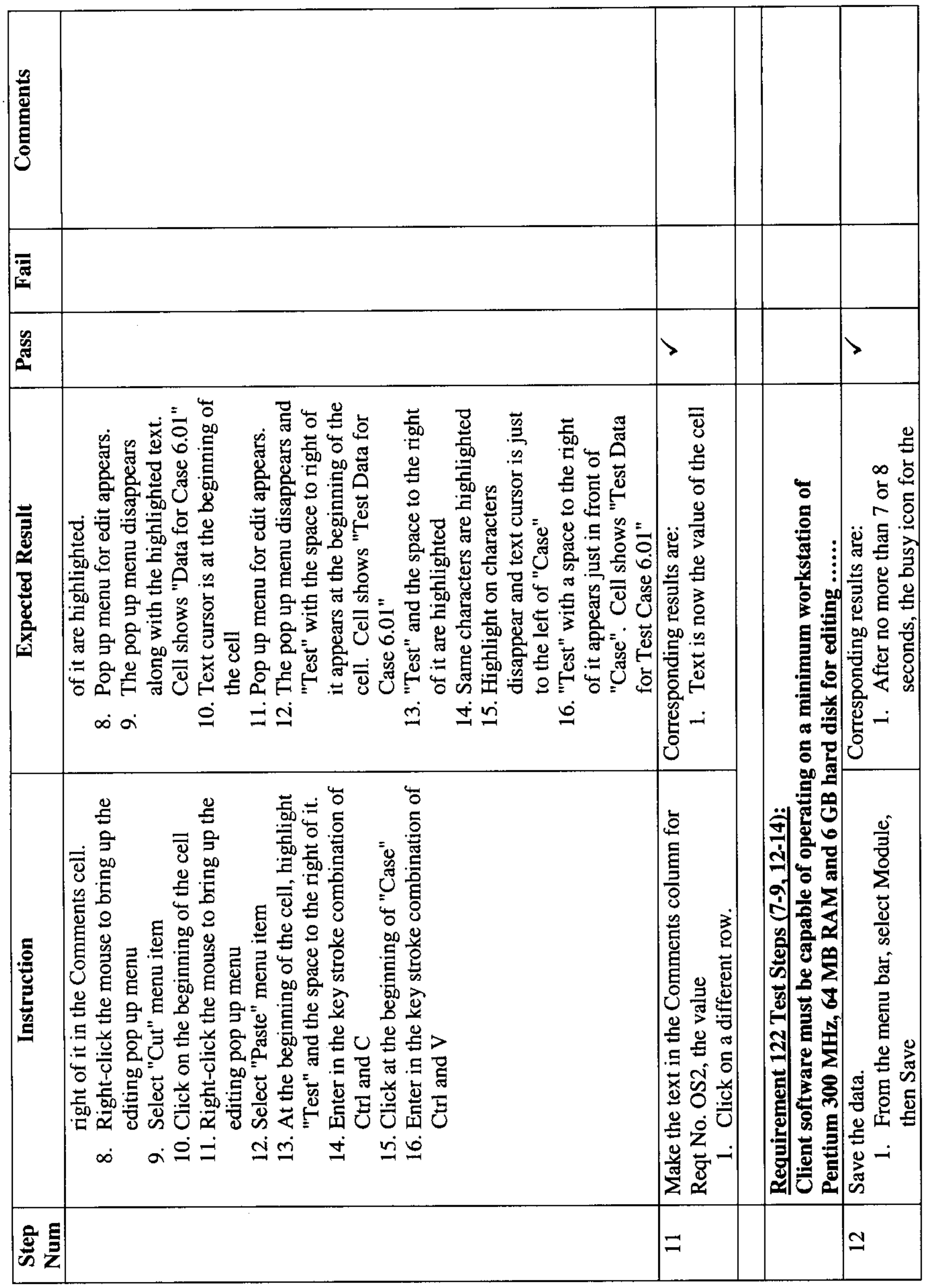


RPP-6251 REV. 0

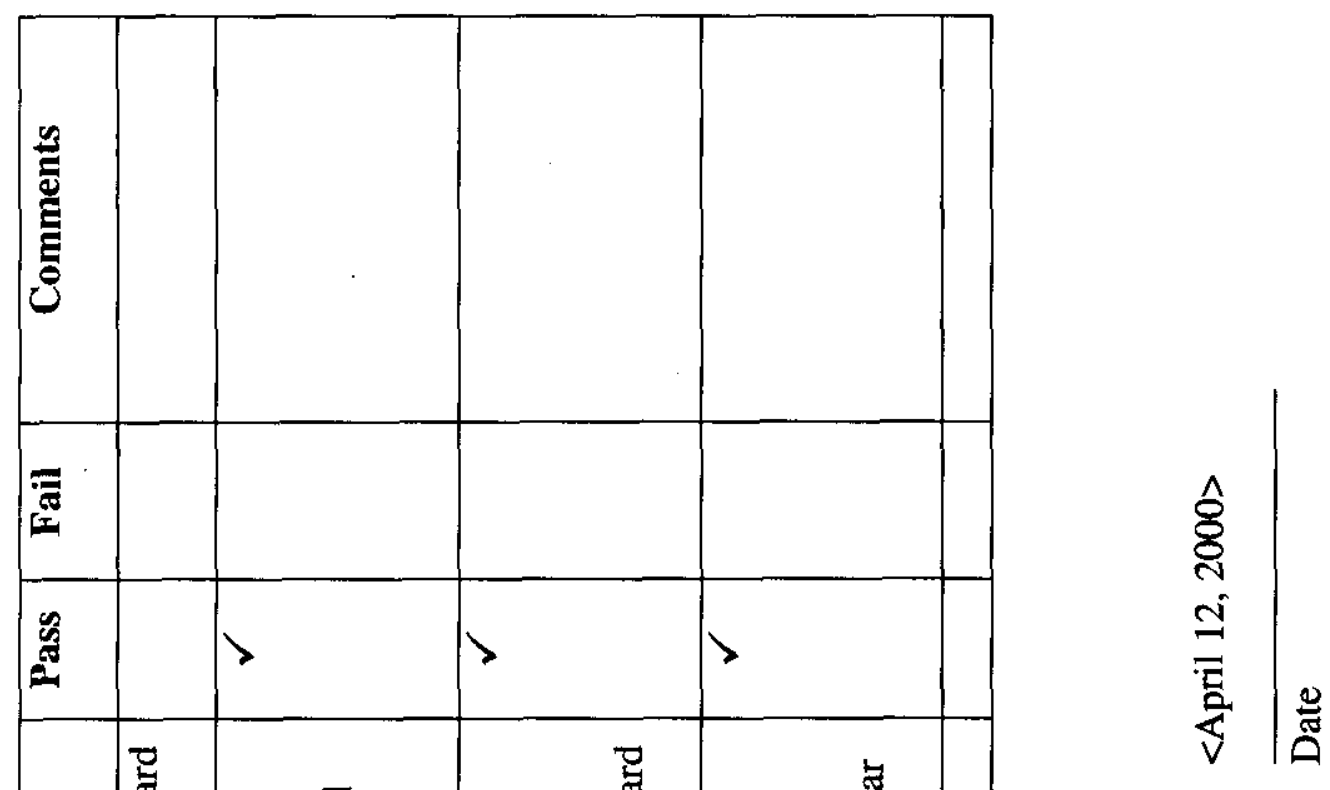


RPP-6251 REV. 0
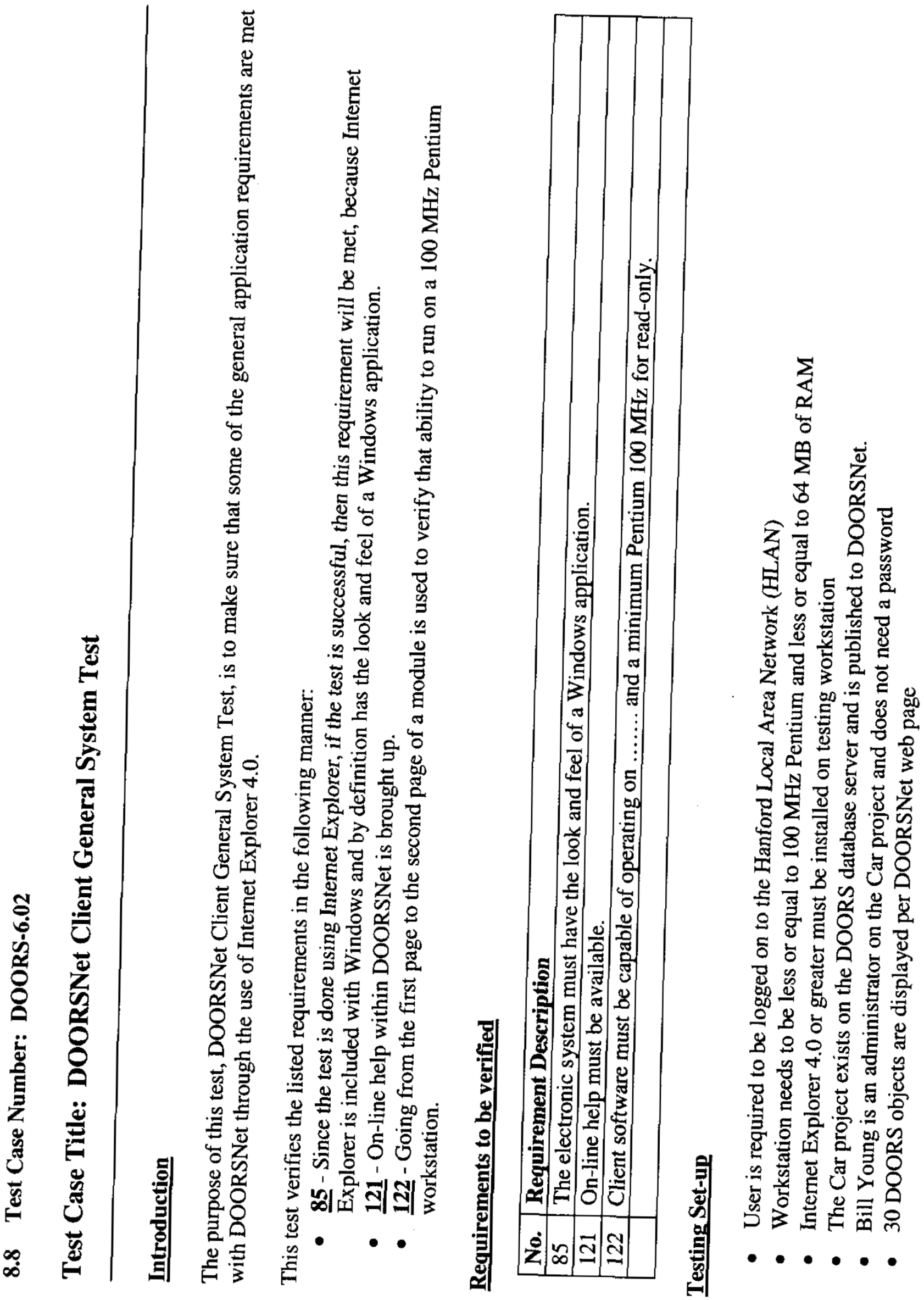
RPP-6251 REV. 0

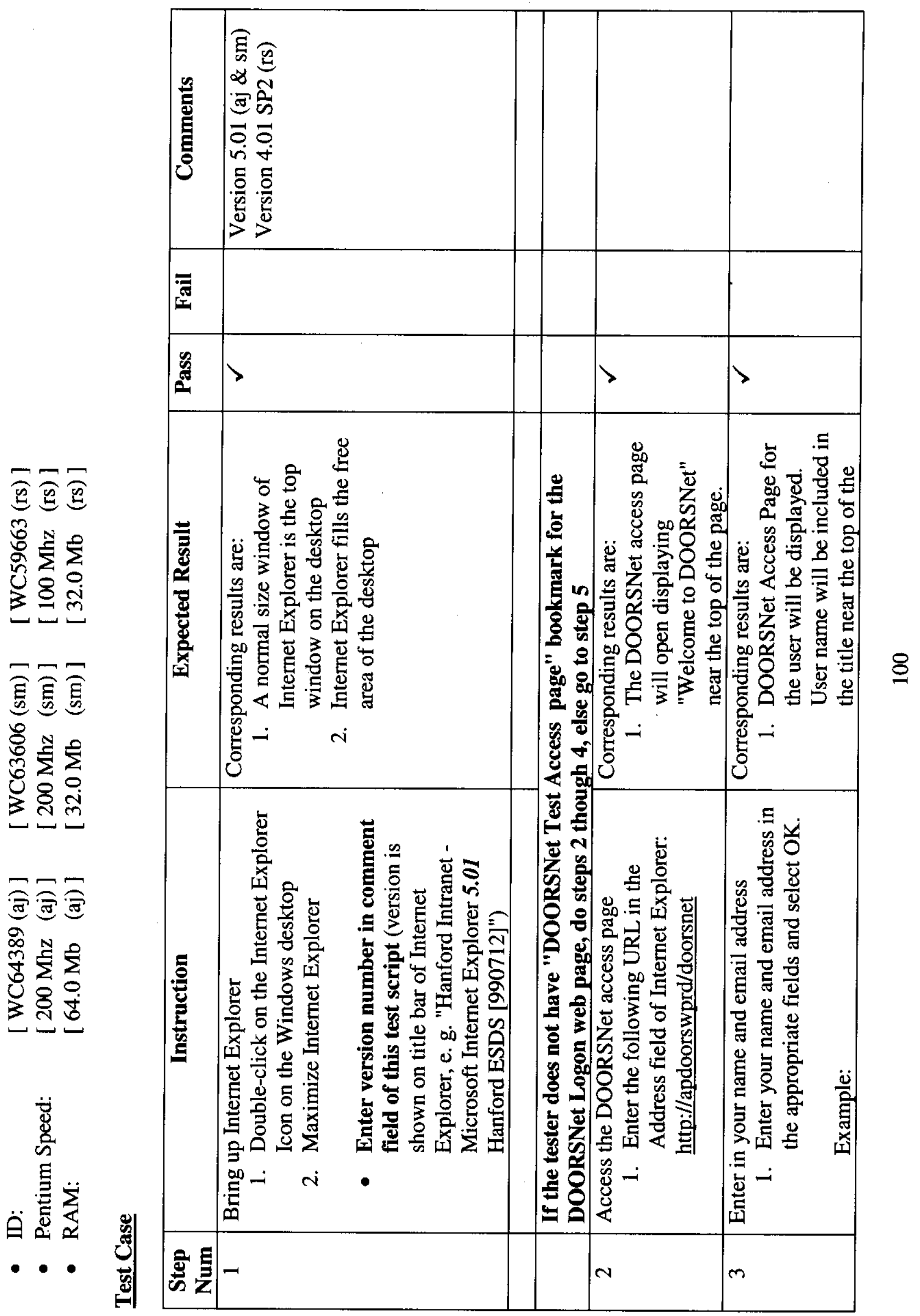




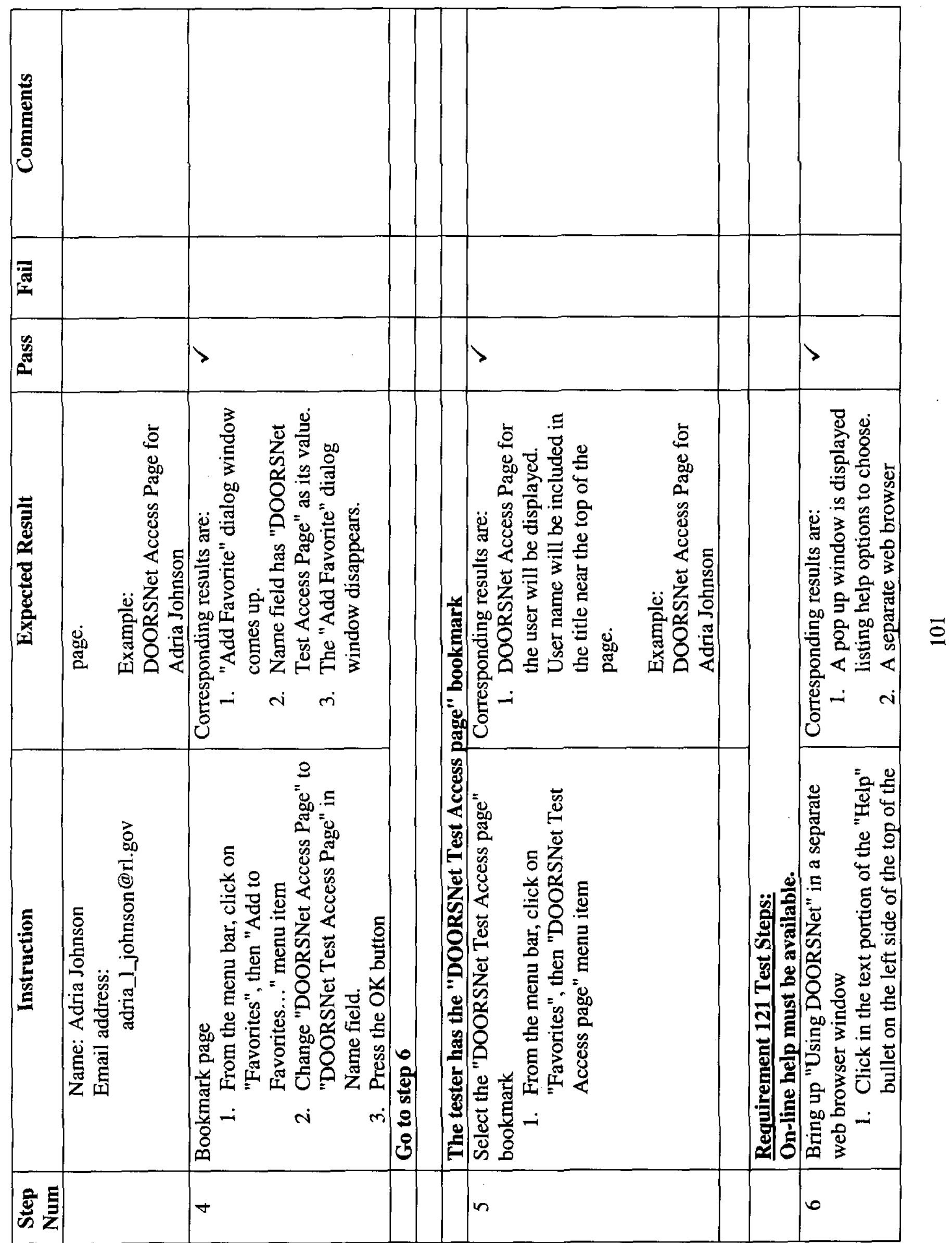


RPP-6251 REV. 0

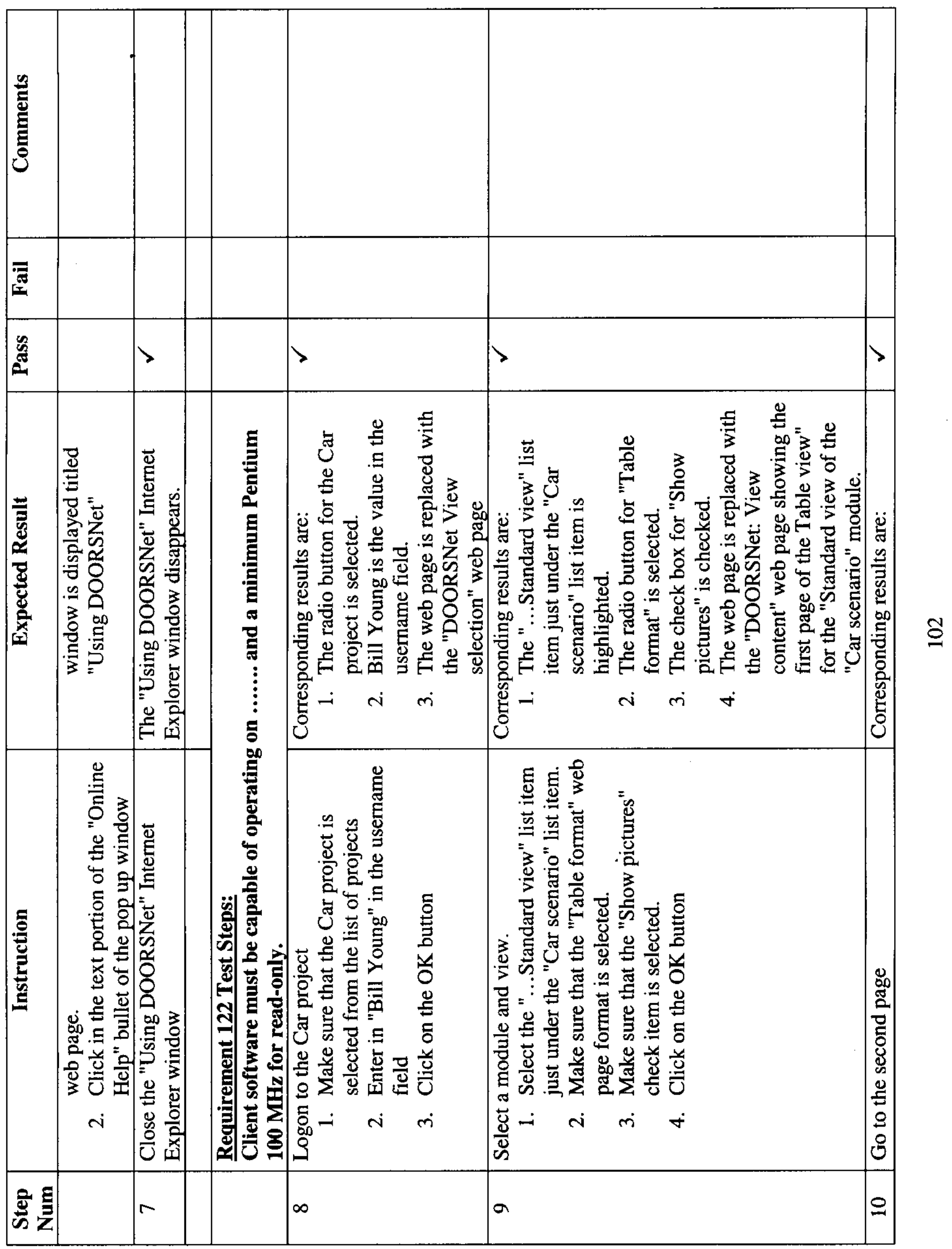


RPP-6251 REV. 0
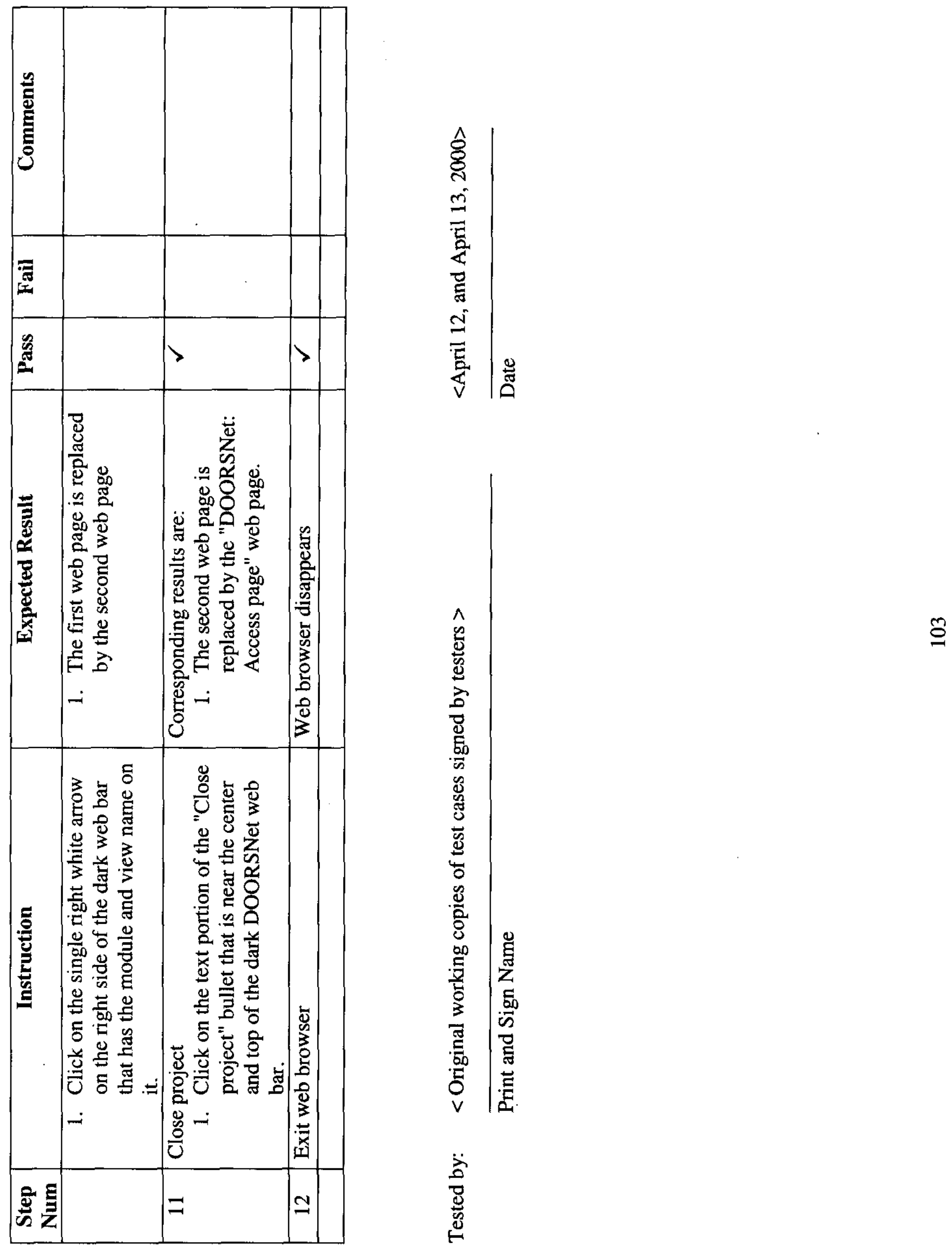


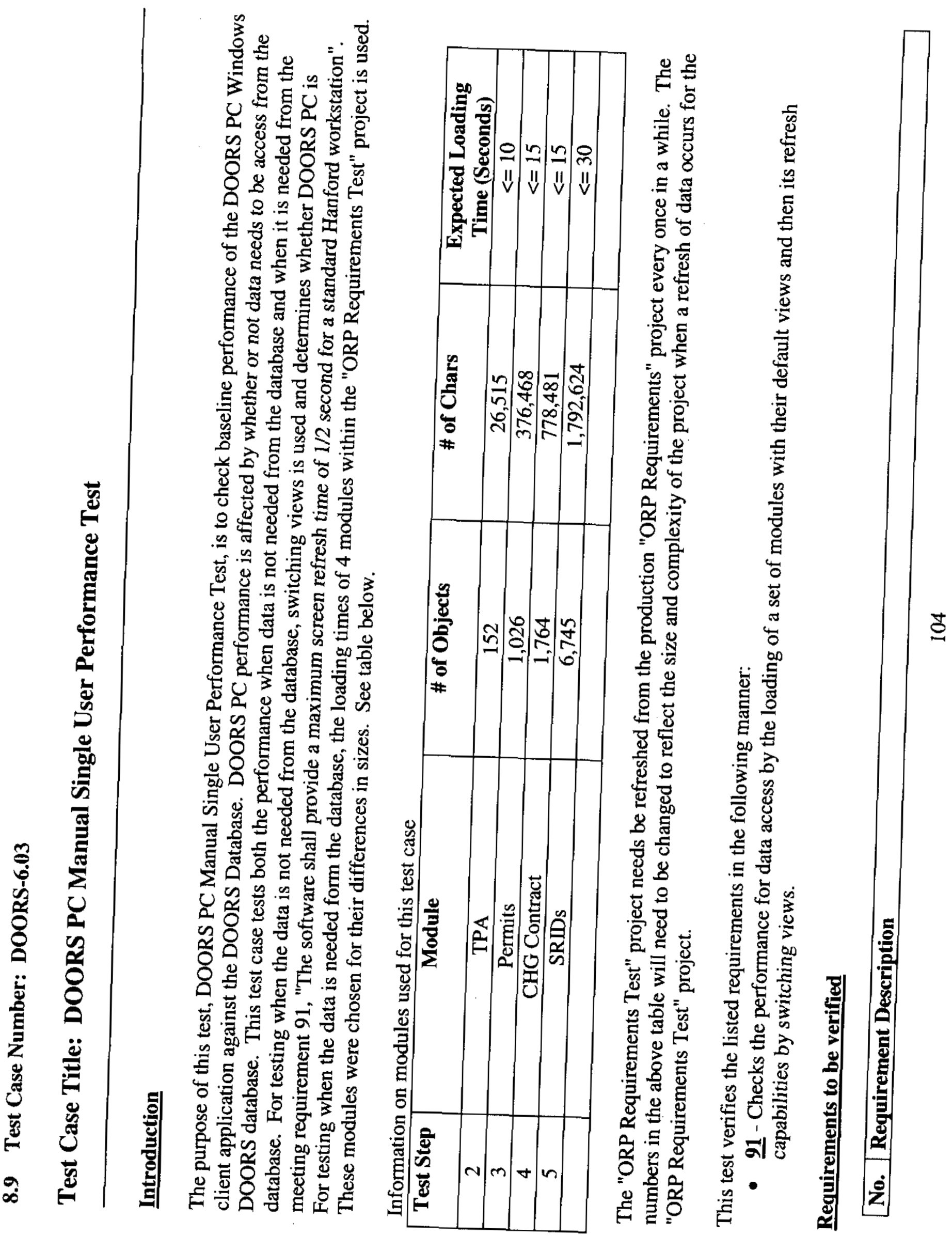




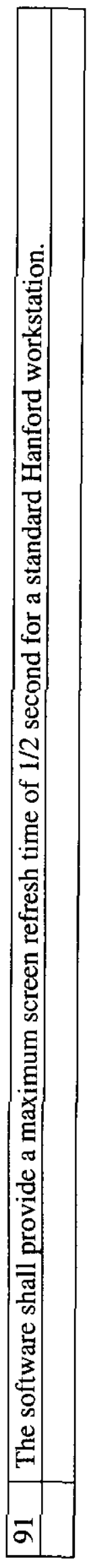

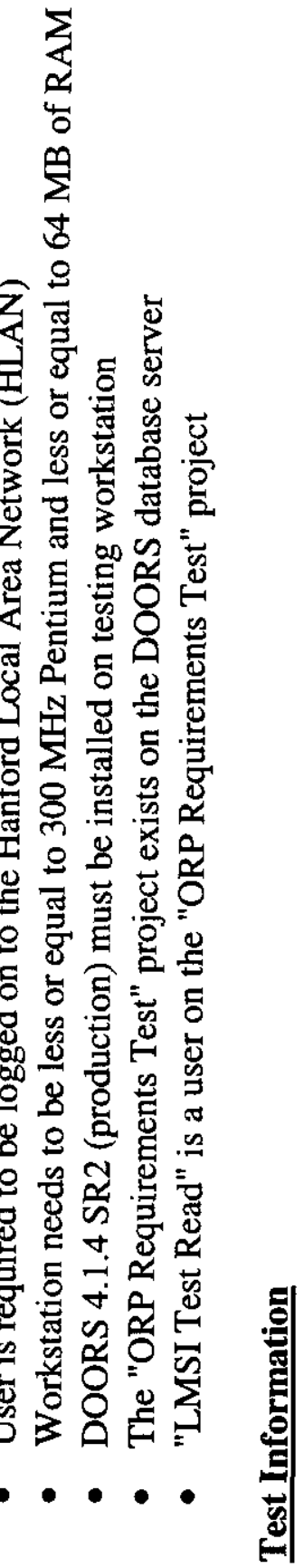
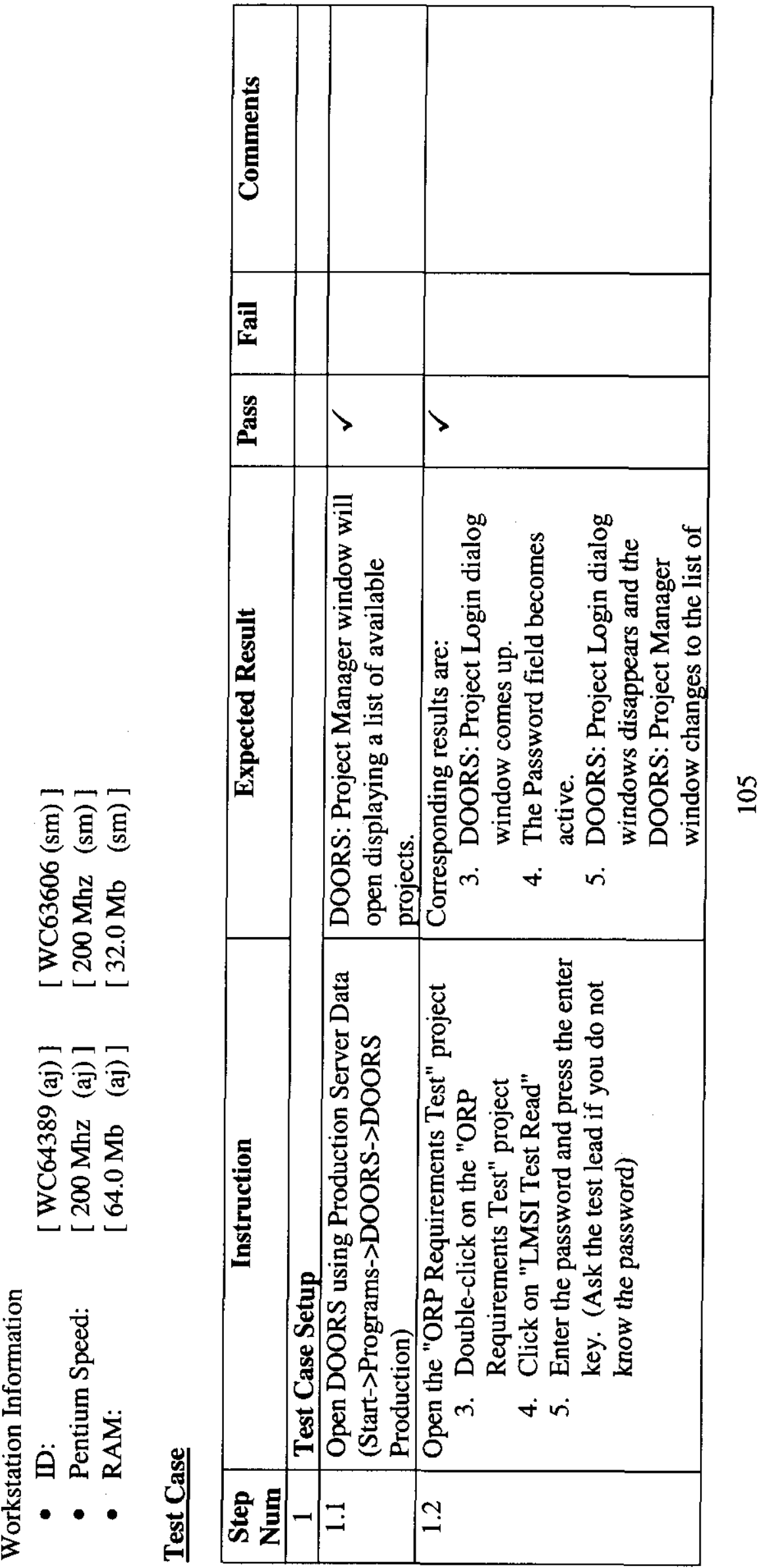
RPP-6251 REV. 0

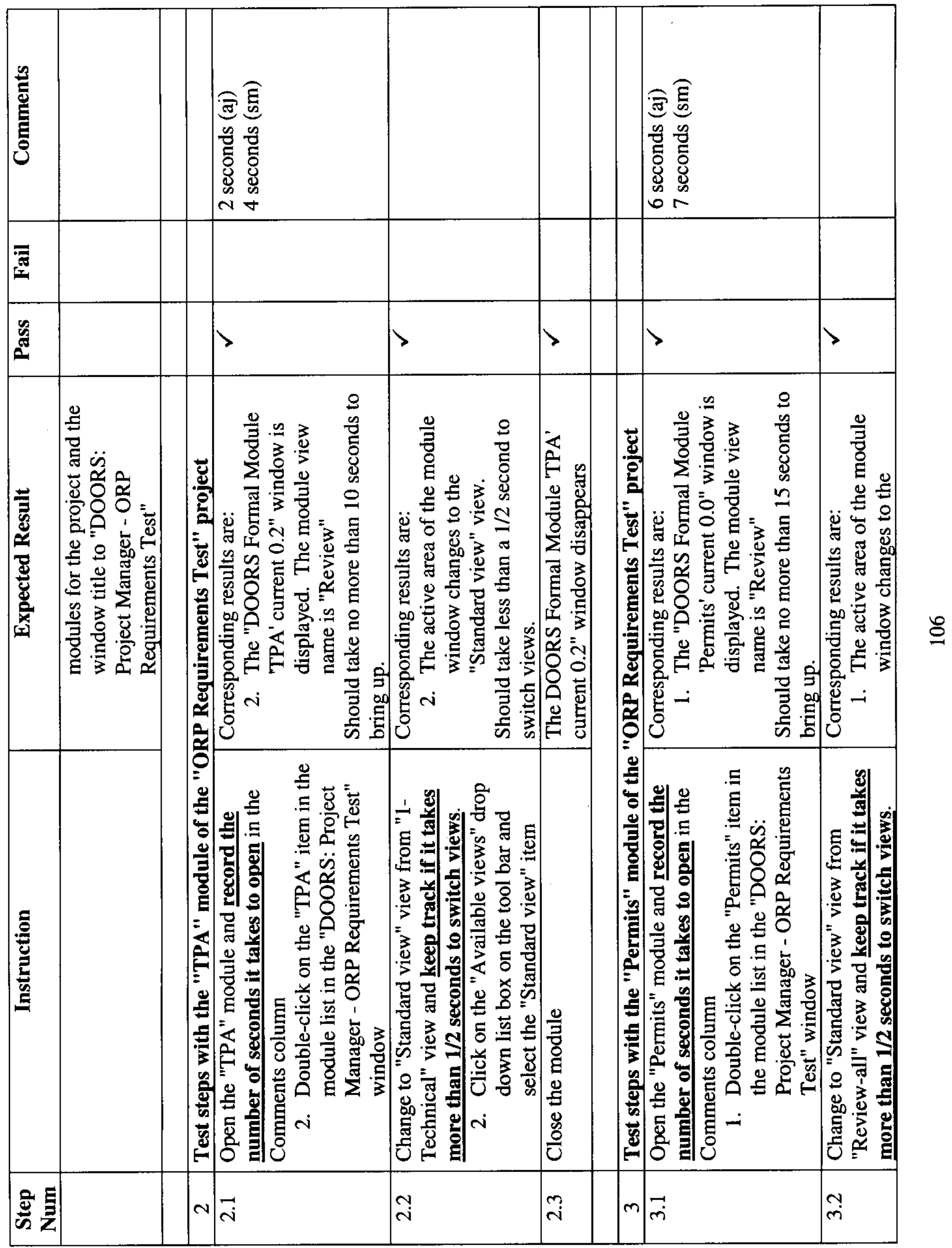


RPP-6251 REV. 0

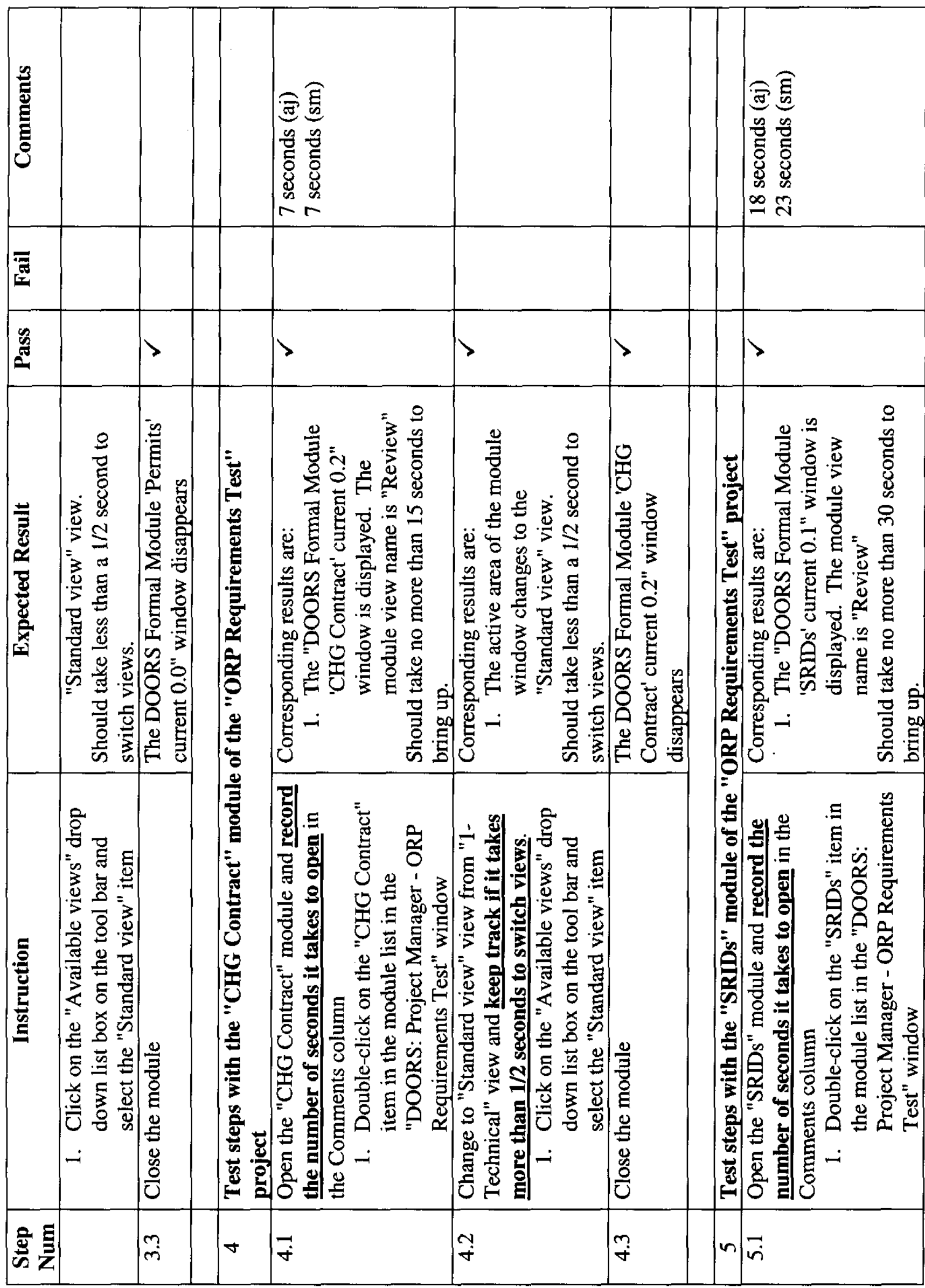


RPP-6251 REV. 0
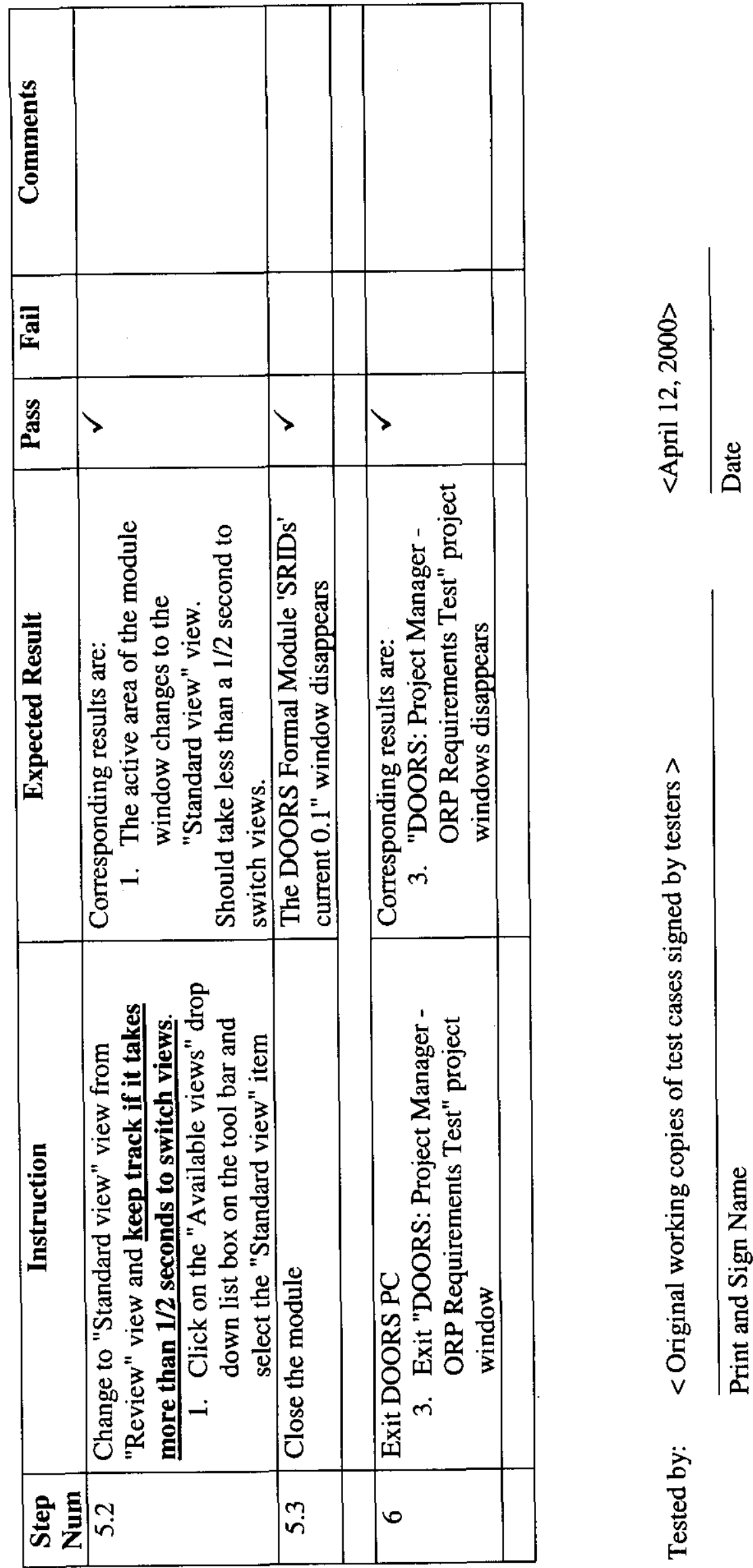


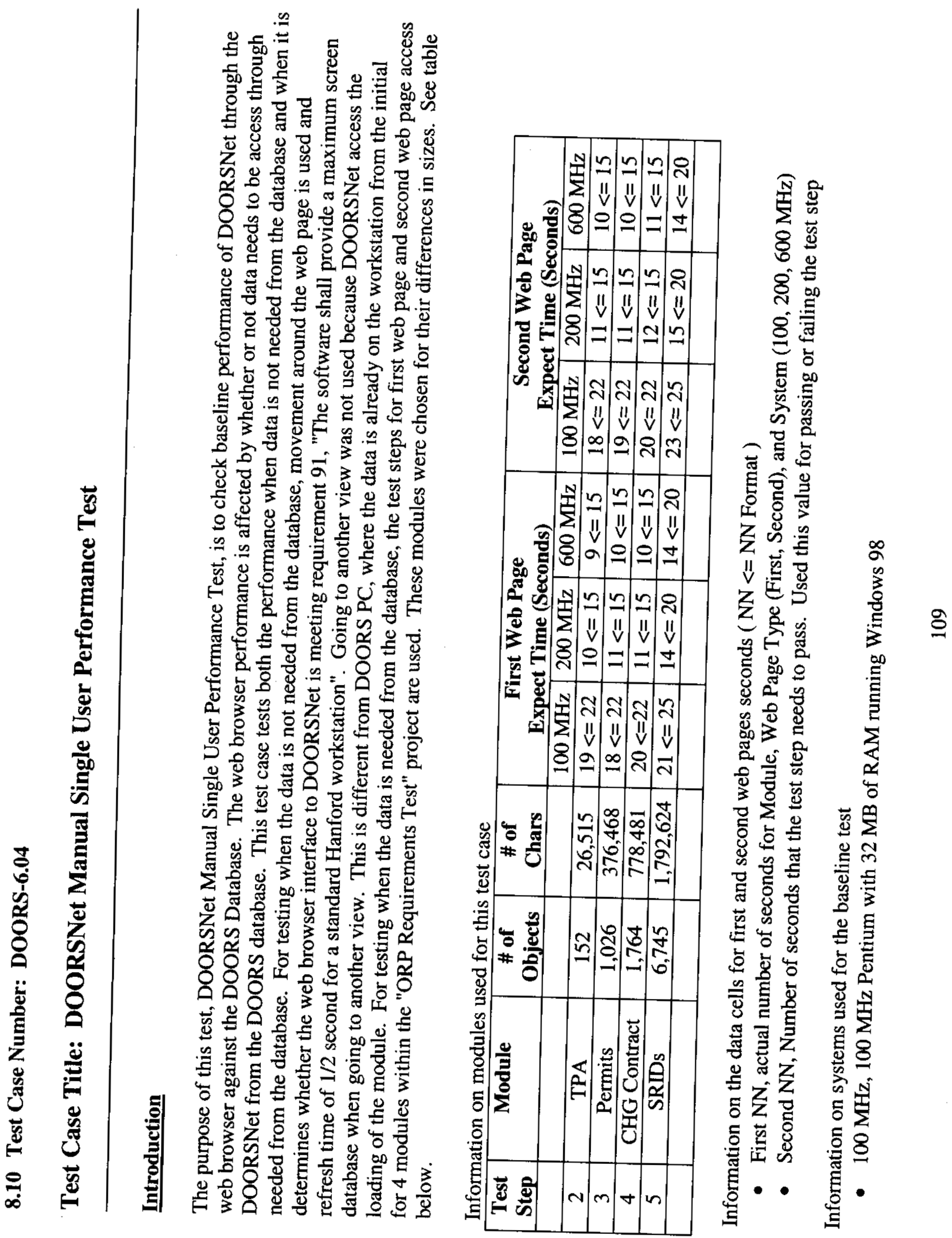




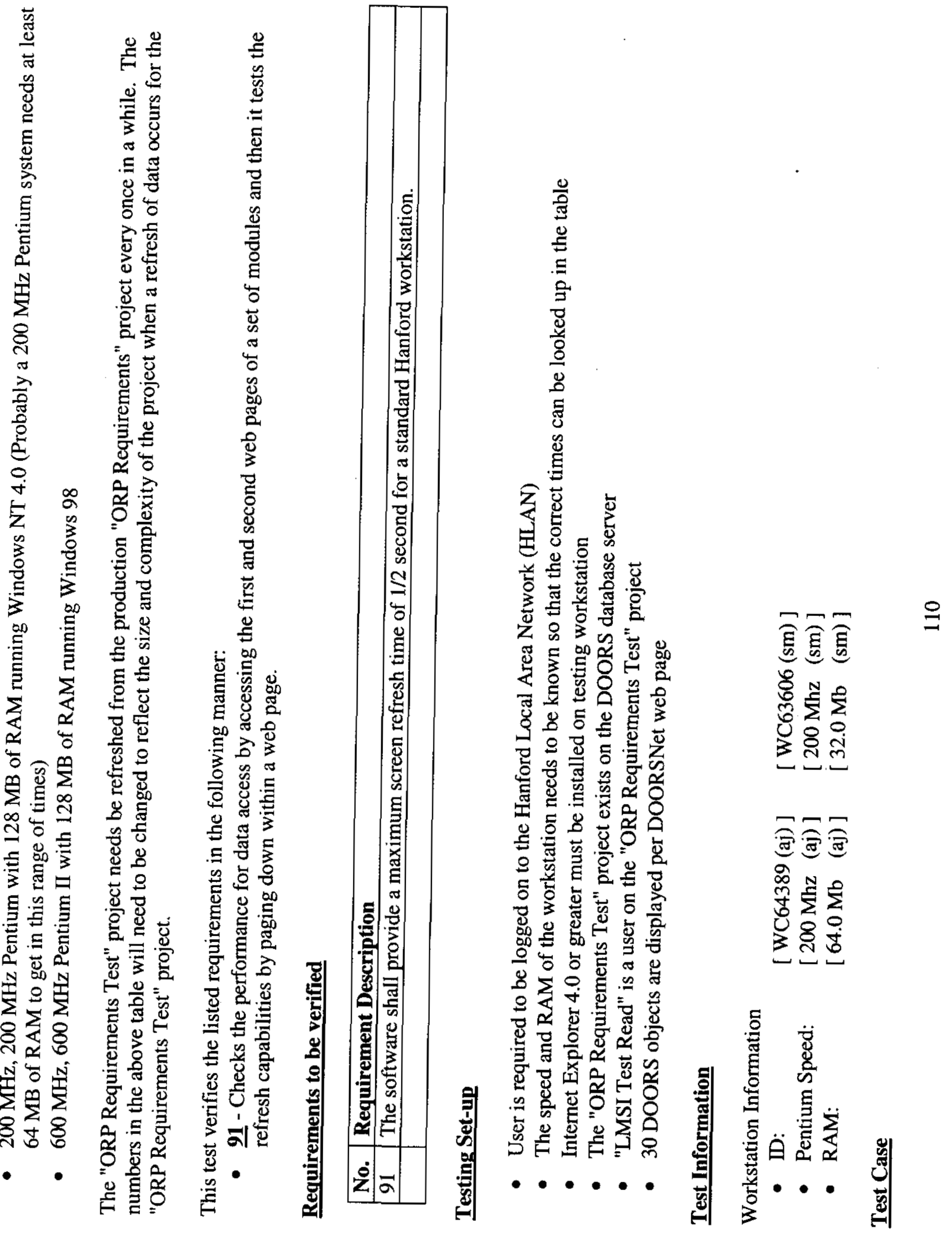


RPP-6251 REV. 0

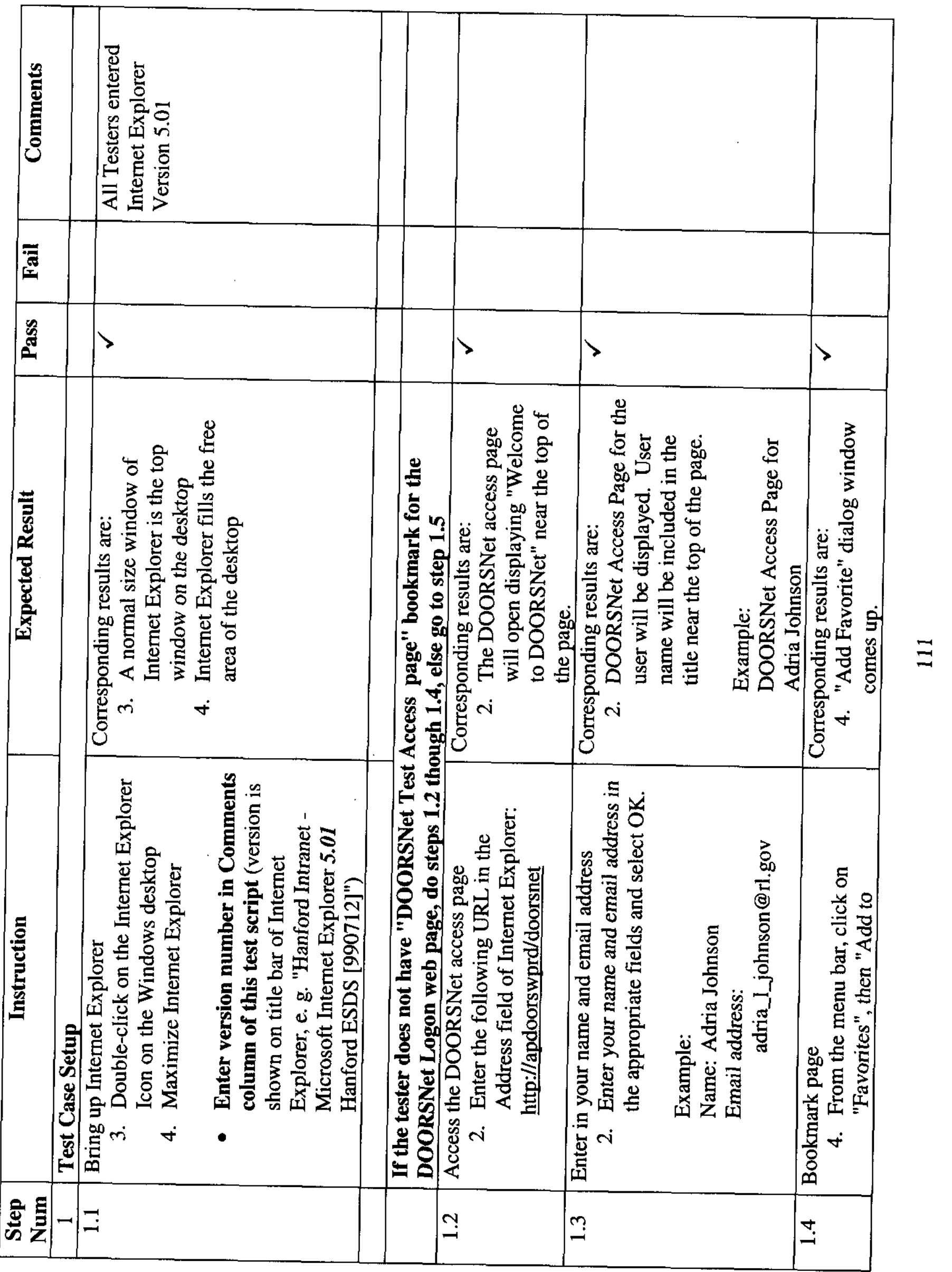


RPP-6251 REV. 0

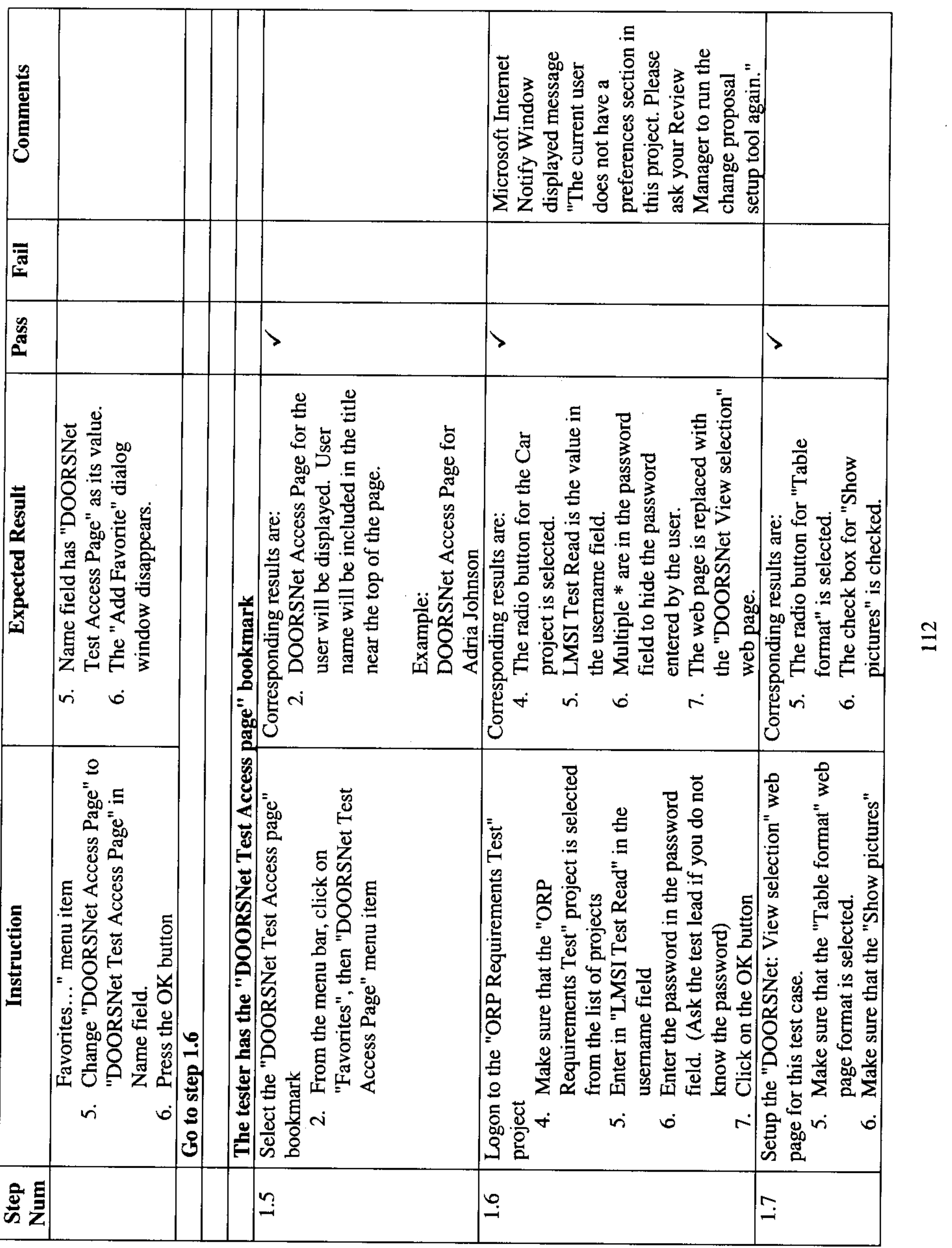


RPP-6251 REV. 0

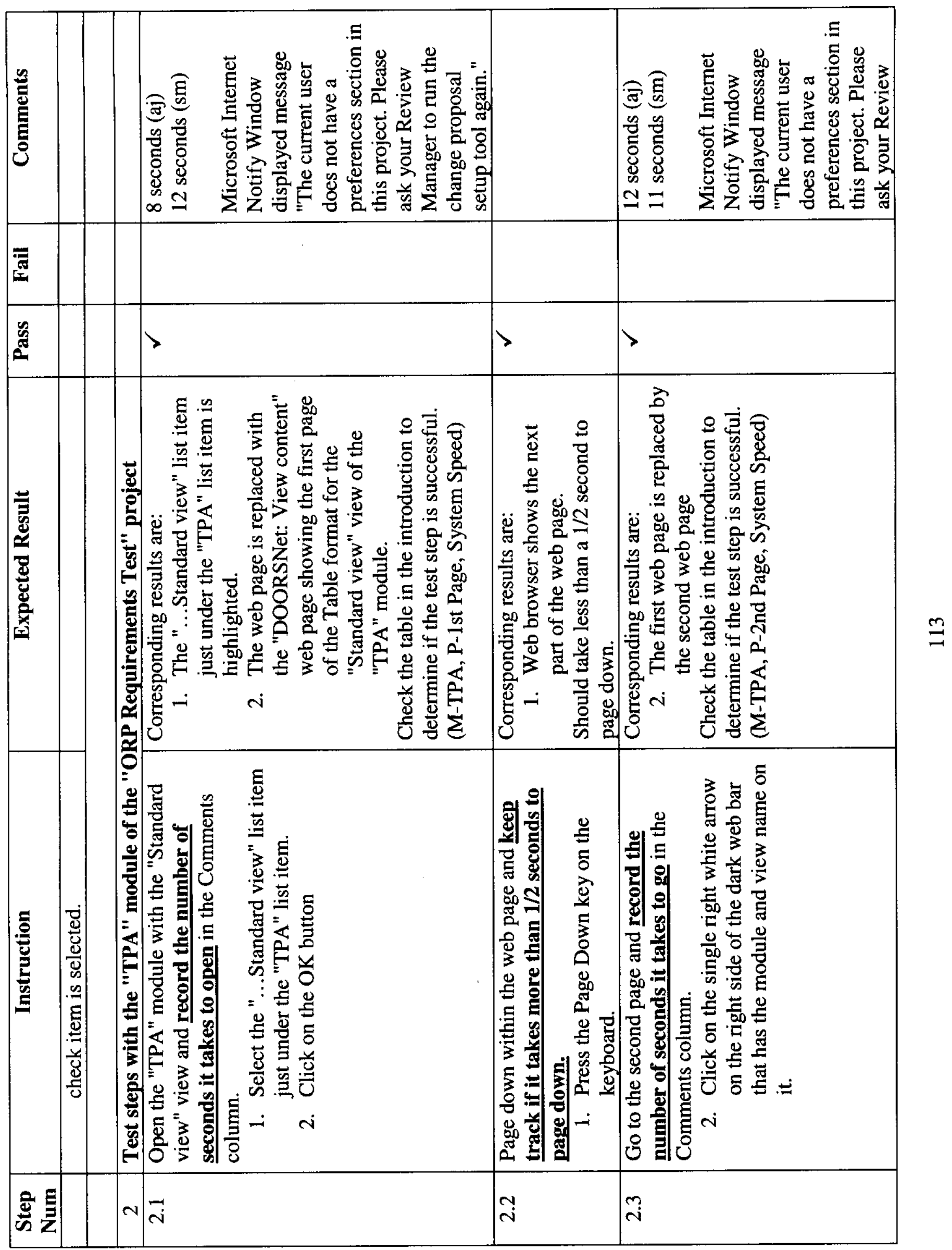




\section{RPP-6251 REV. 0}

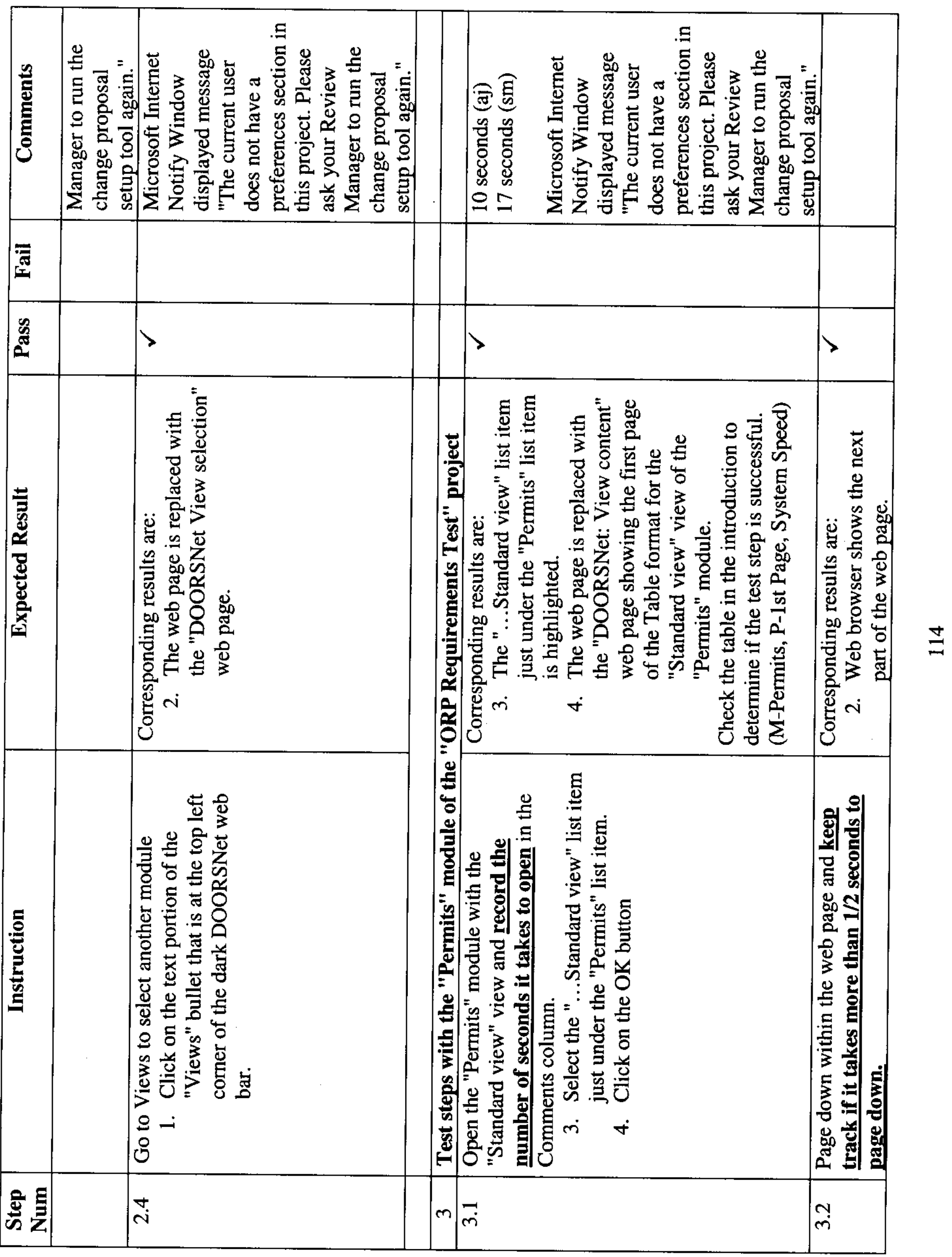


RPP-6251 REV. 0

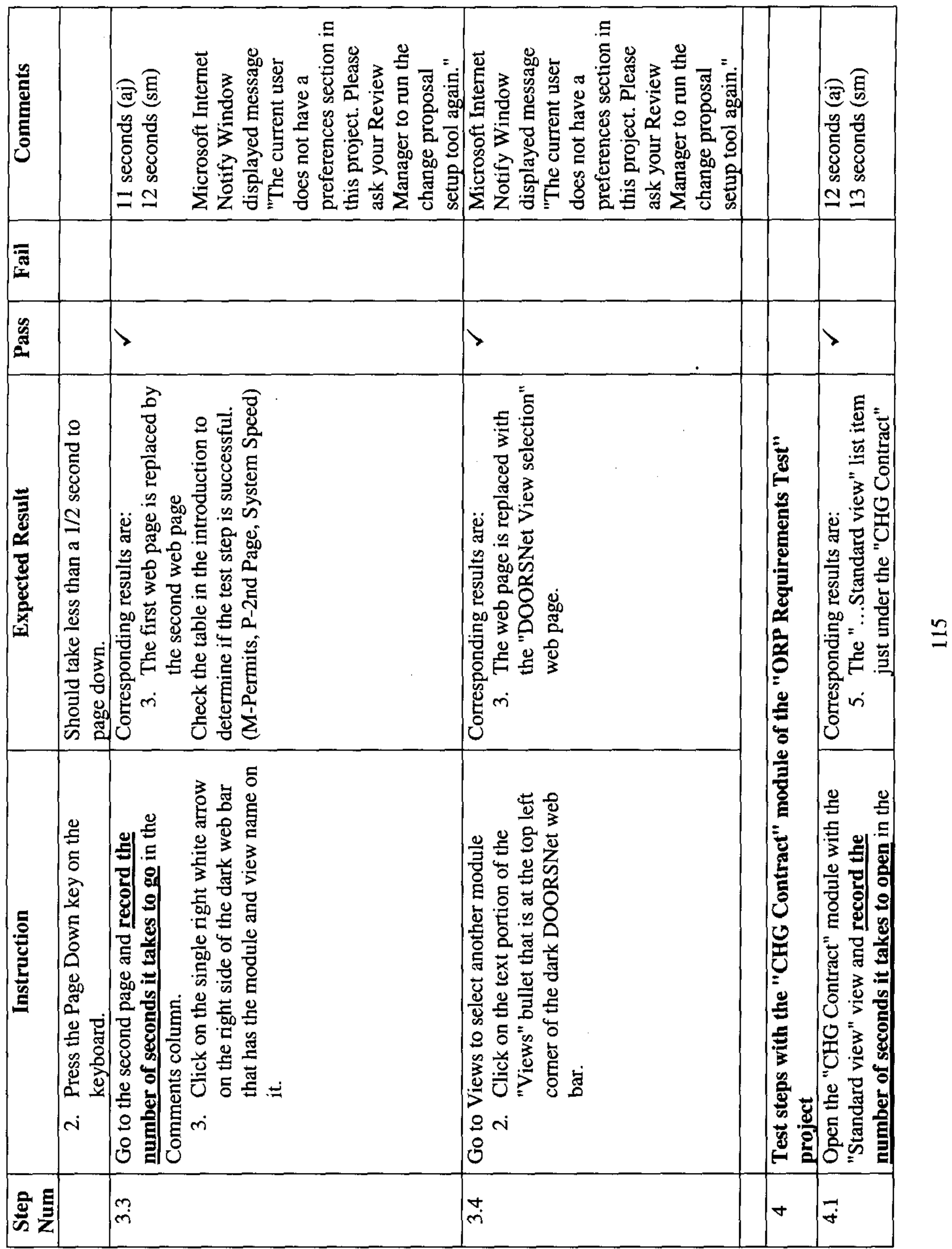




\section{RPP-6251 REV. 0}

\begin{tabular}{|c|c|c|c|c|}
\hline & 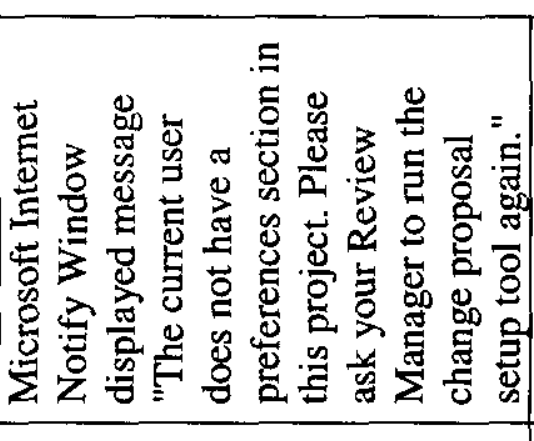 & & \multicolumn{2}{|c|}{ 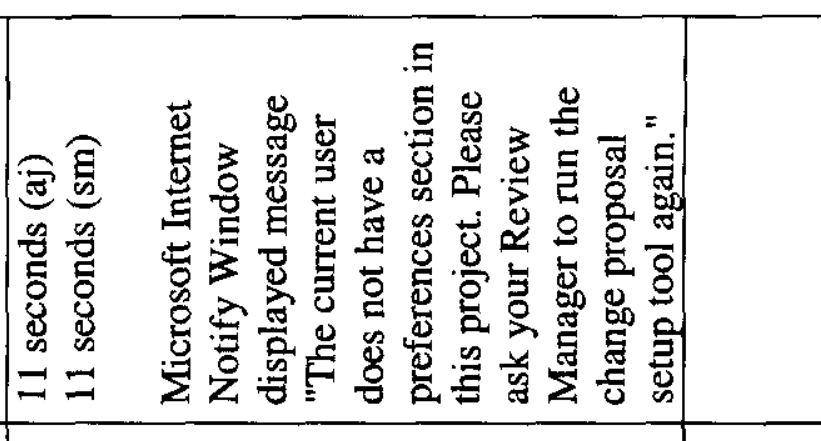 } \\
\hline $\bar{F}$ & & & & \\
\hline 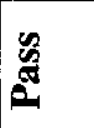 & & \rangle & $>$ & $>$ \\
\hline & 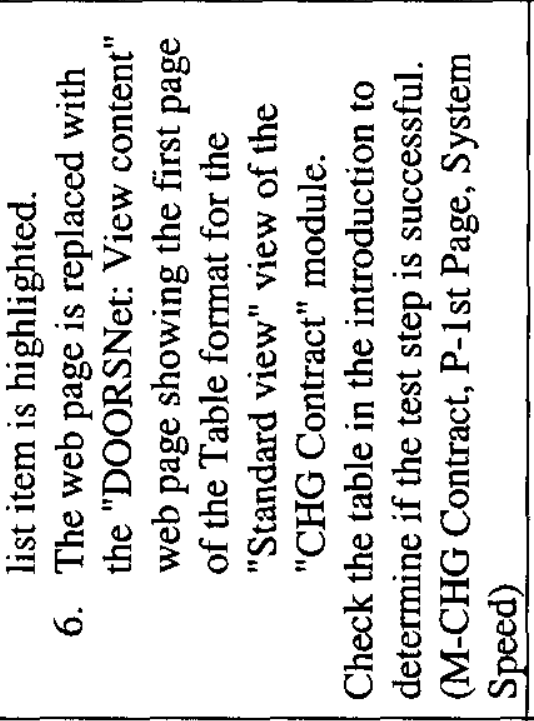 & 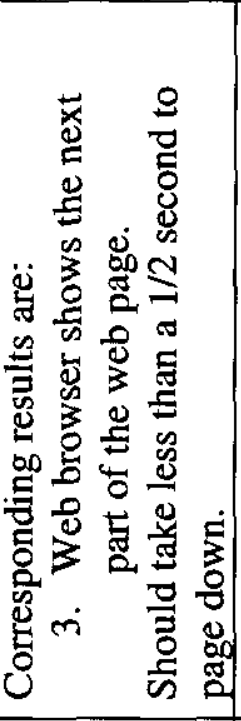 & 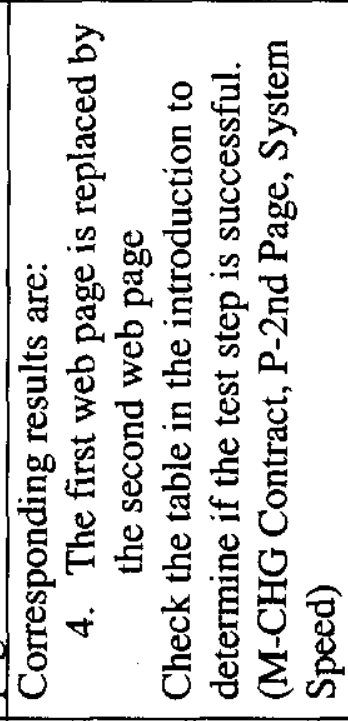 & 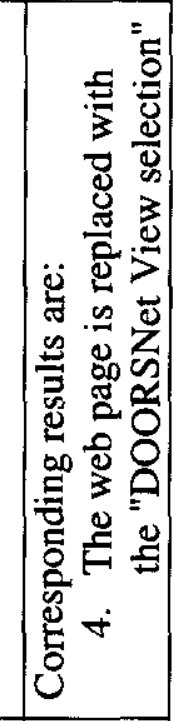 \\
\hline & 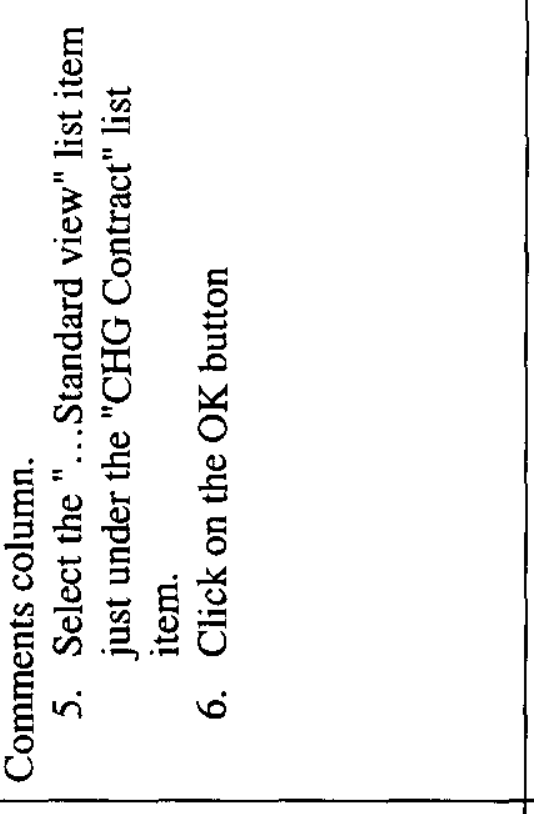 & 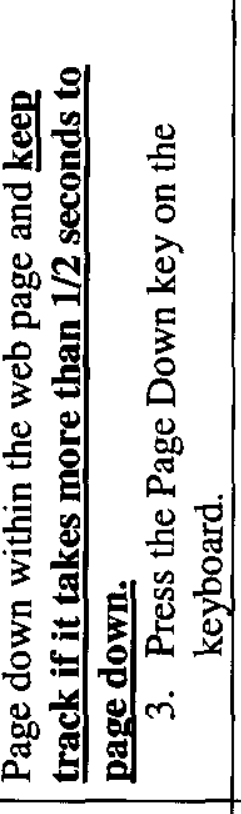 & 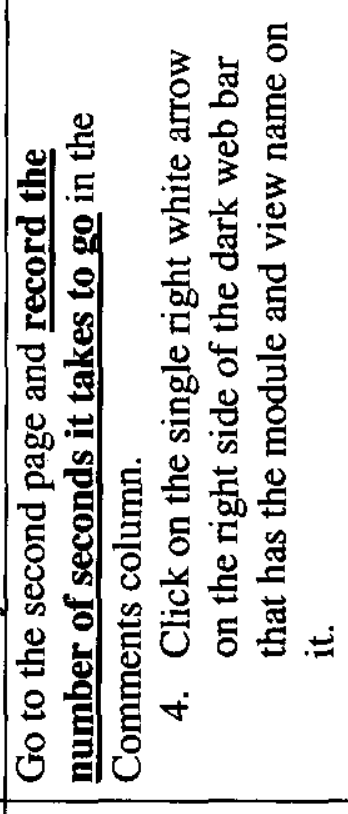 & 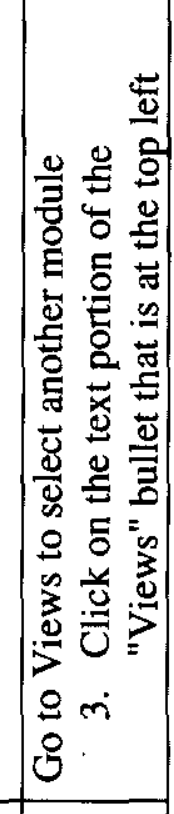 \\
\hline $\bar{z}$ & & + & $\stackrel{m}{*}$ & $\stackrel{+}{+}$ \\
\hline
\end{tabular}


RPP-6251 REV. 0

\begin{tabular}{|c|c|c|c|c|c|c|c|c|}
\hline 总 & & & 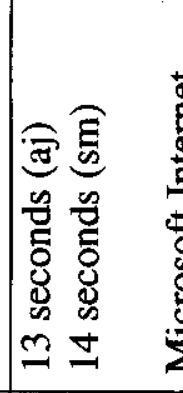 & 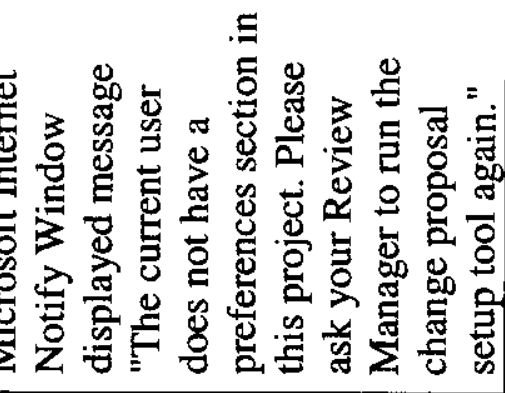 & & & 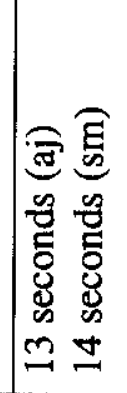 & 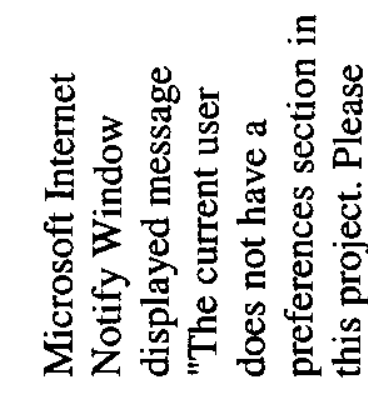 \\
\hline 疍 & & & & & & & & \\
\hline $\begin{array}{l}y \\
z \\
z\end{array}$ & & & $>$ & & $>$ & & $>$ & \\
\hline 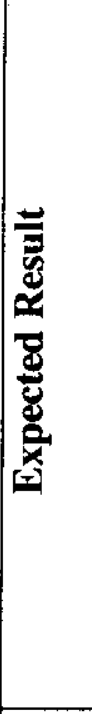 & 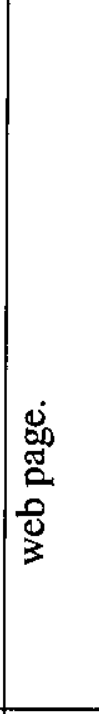 & 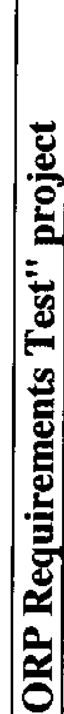 & 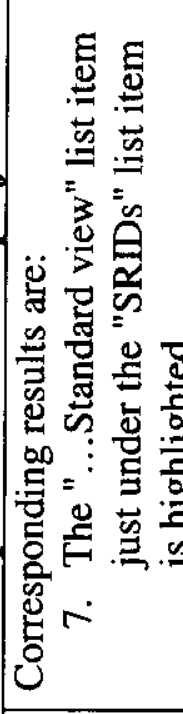 & 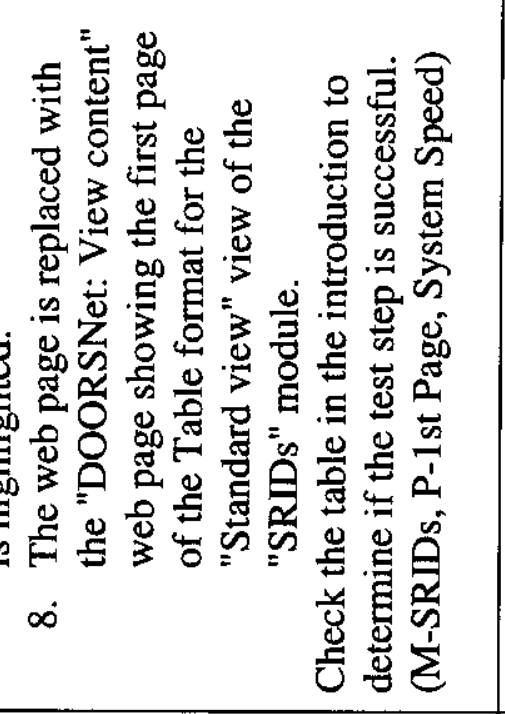 & 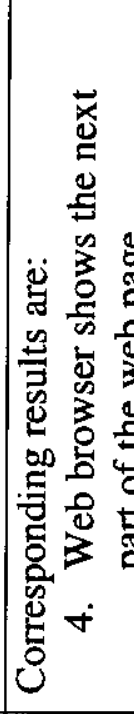 & 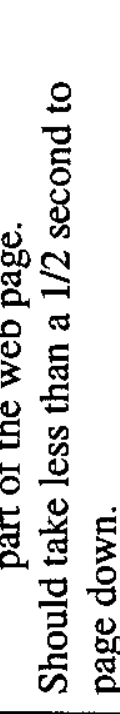 & 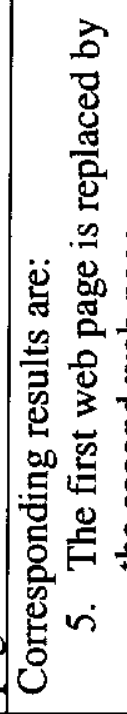 & 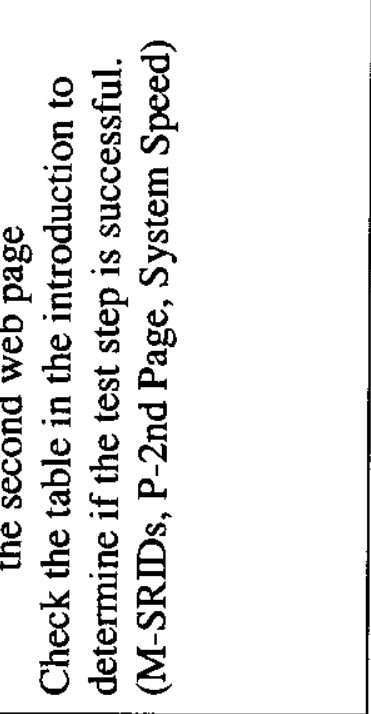 \\
\hline 总 & 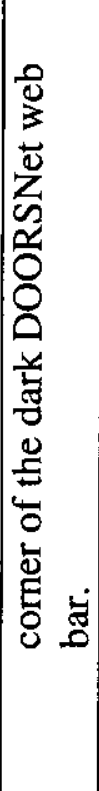 & 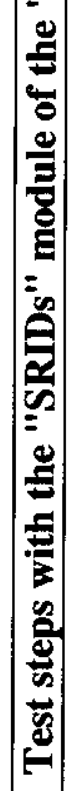 & 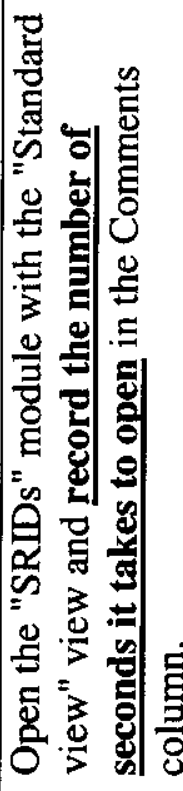 & 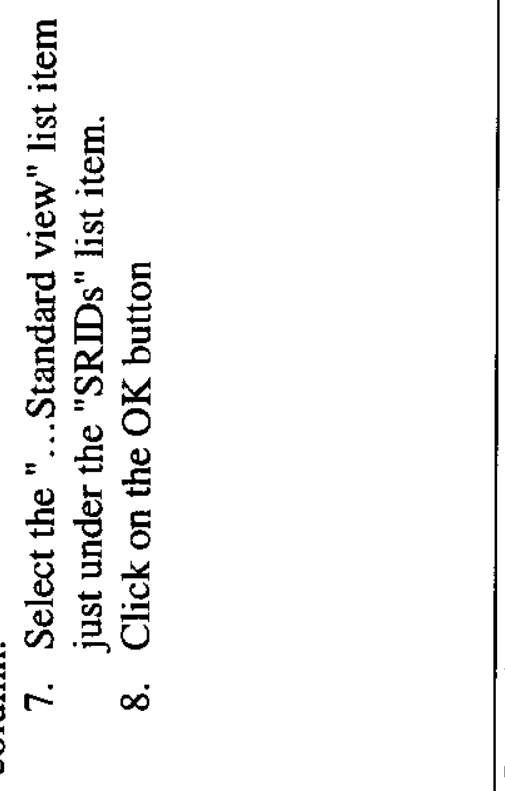 & 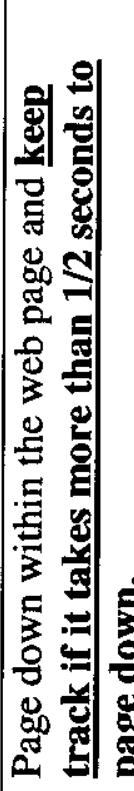 & 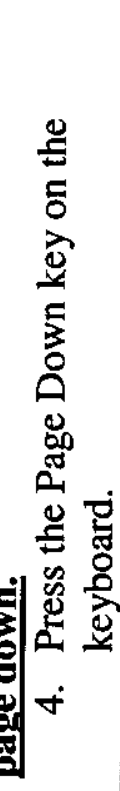 & 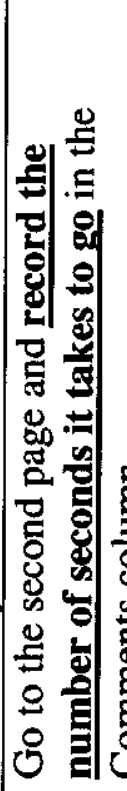 & 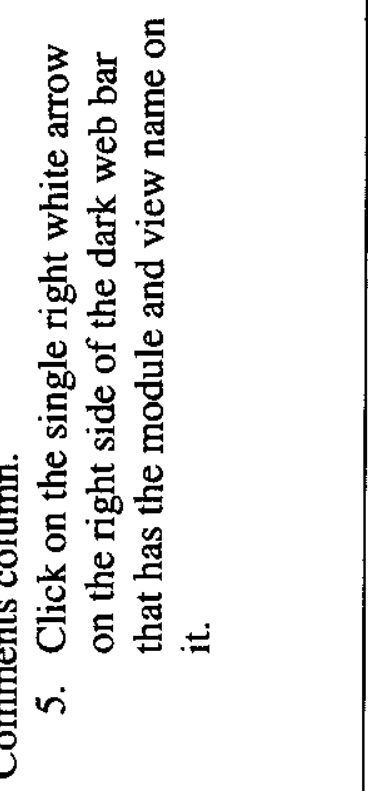 \\
\hline 竎焉 & & $n$ & $\vec{v}$ & & ִָ & & $m$ & \\
\hline
\end{tabular}


RPP-6251 REV. 0
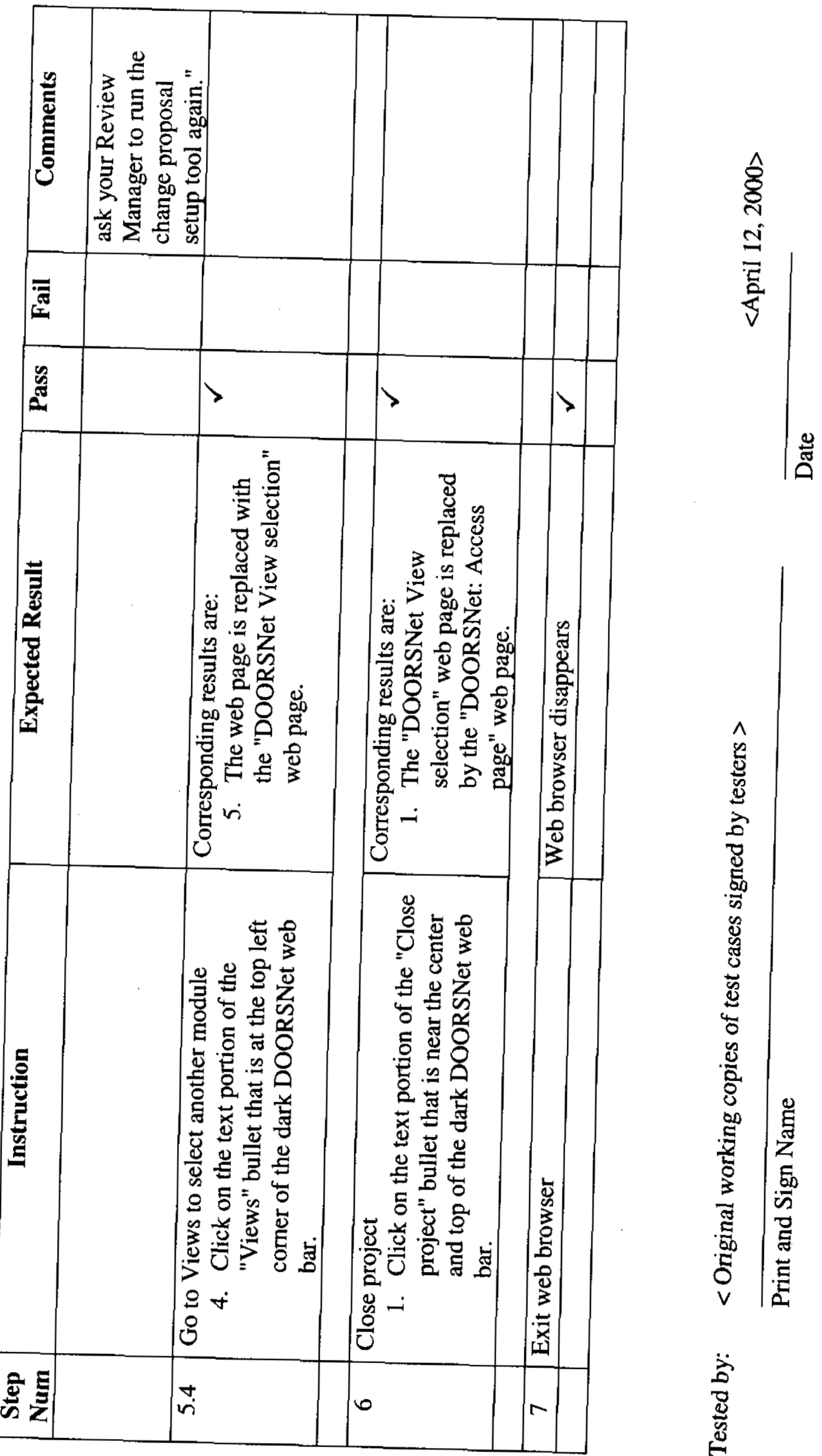
RPP-6251 REV. 0

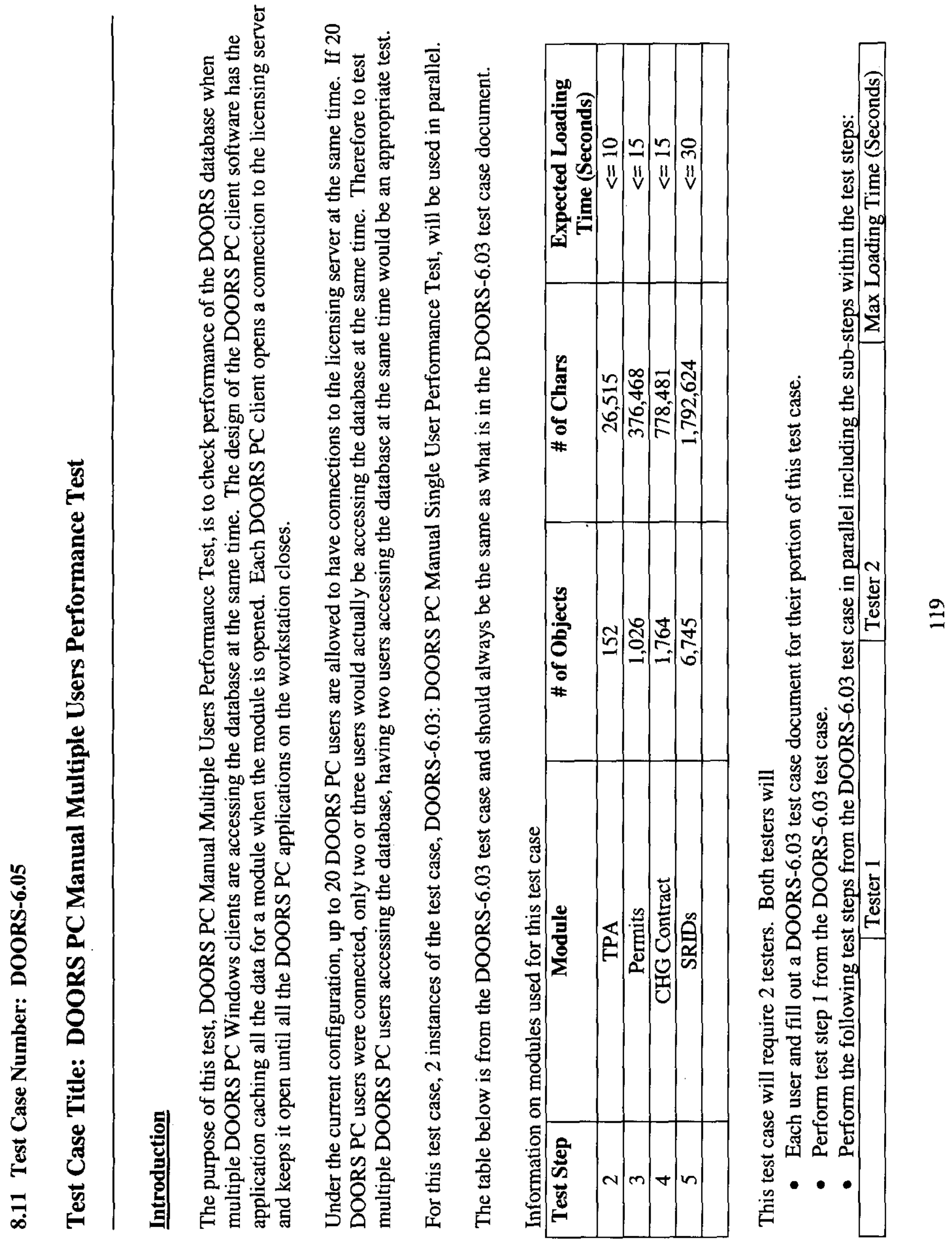




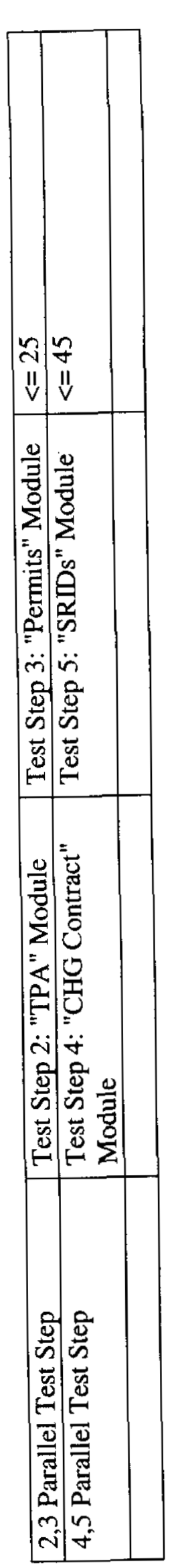

뭉
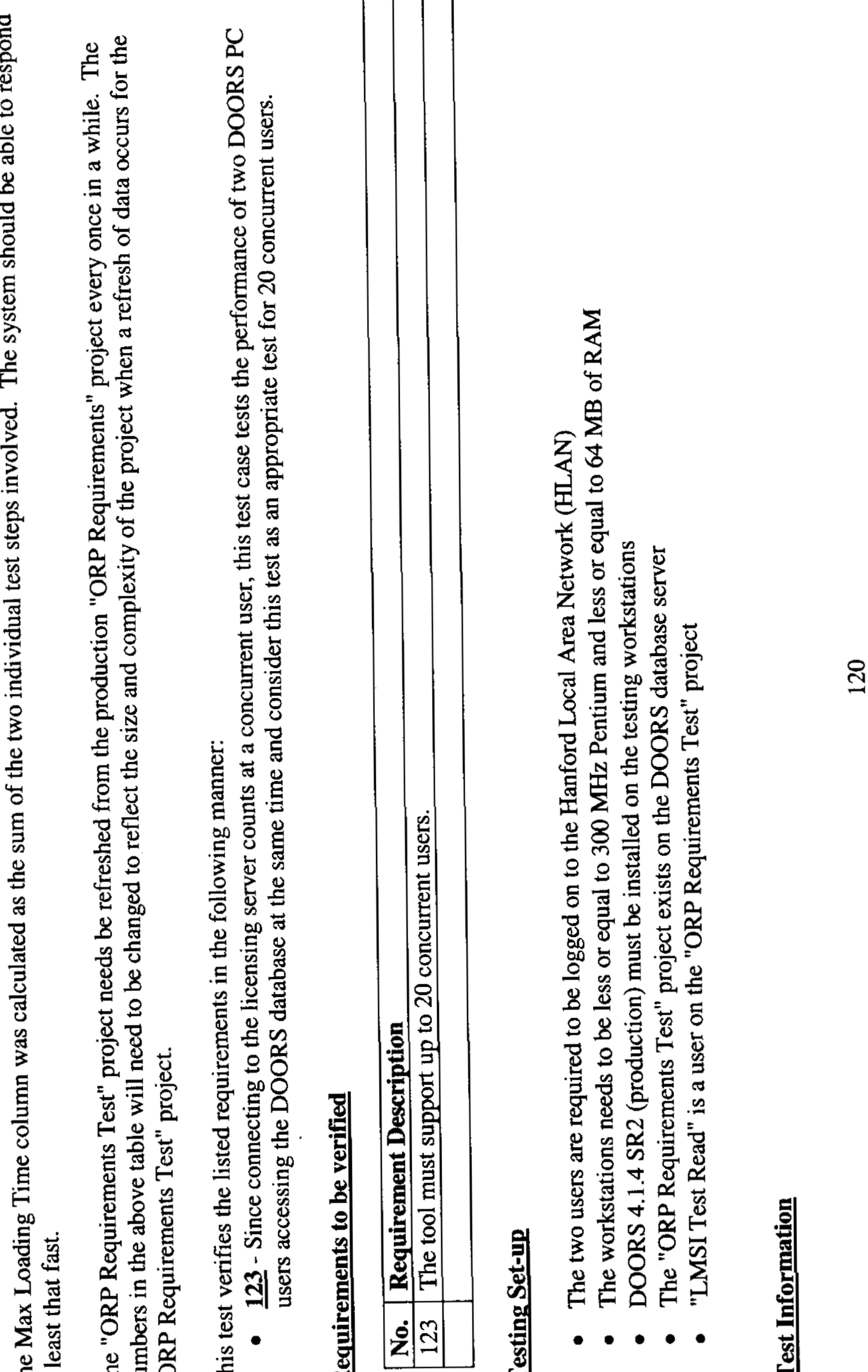

是苛的范

홍용

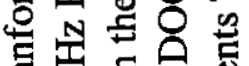

昰实。

옹현

○蛋芯

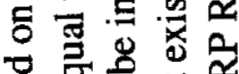

总

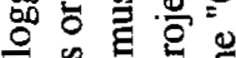

密吉至

용

马워용

骂。苛

马्ष

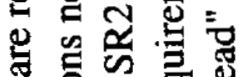

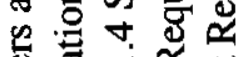

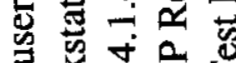

임

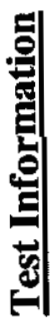


RPP-6251 REV. 0
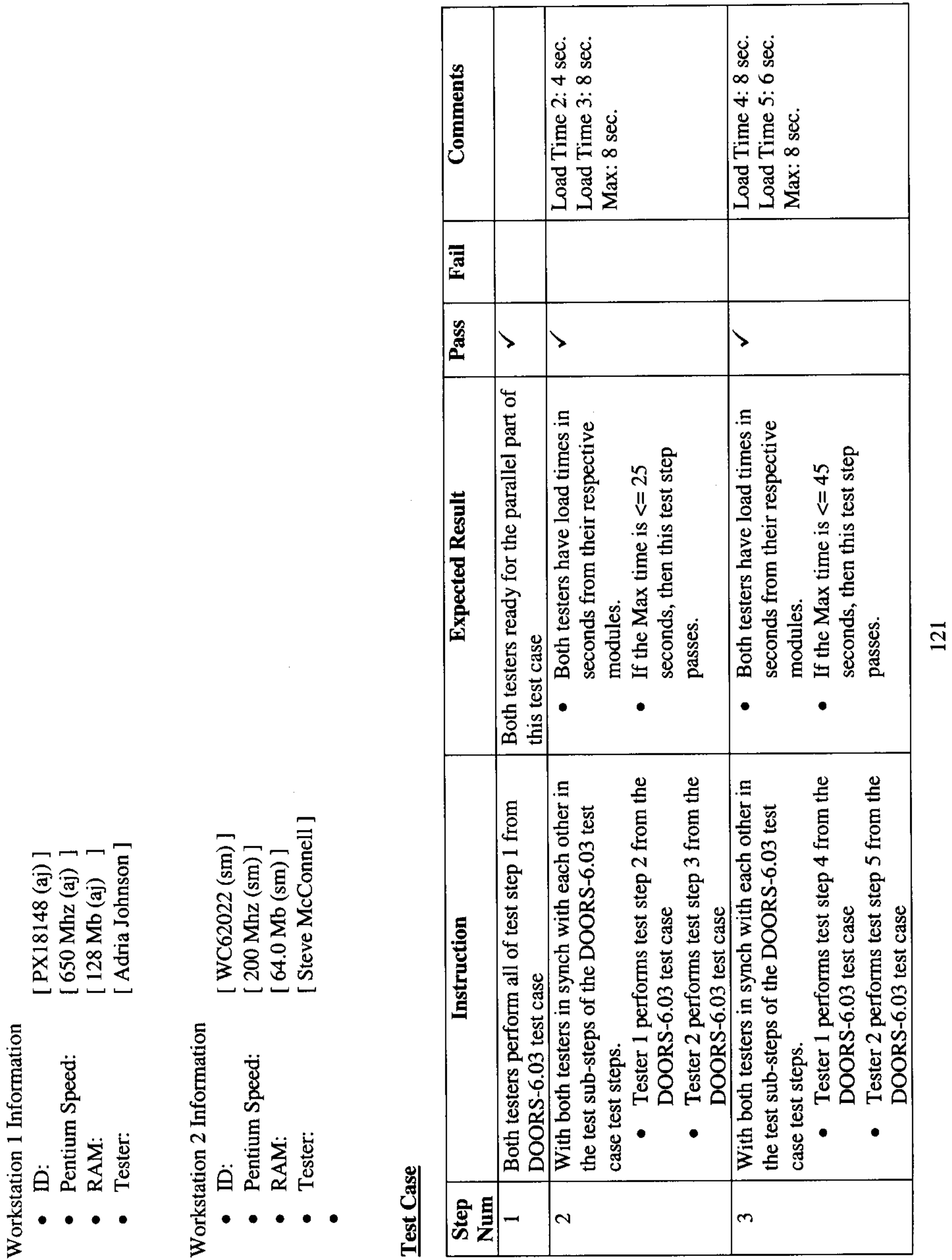
RPP-6251 REV. 0

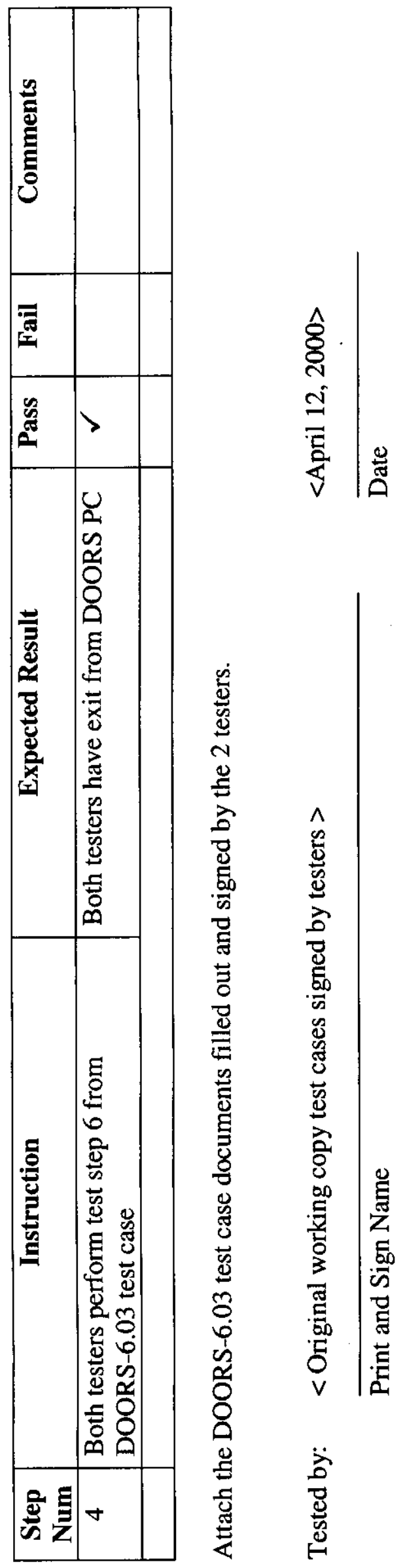


RPP-6251 REV. 0

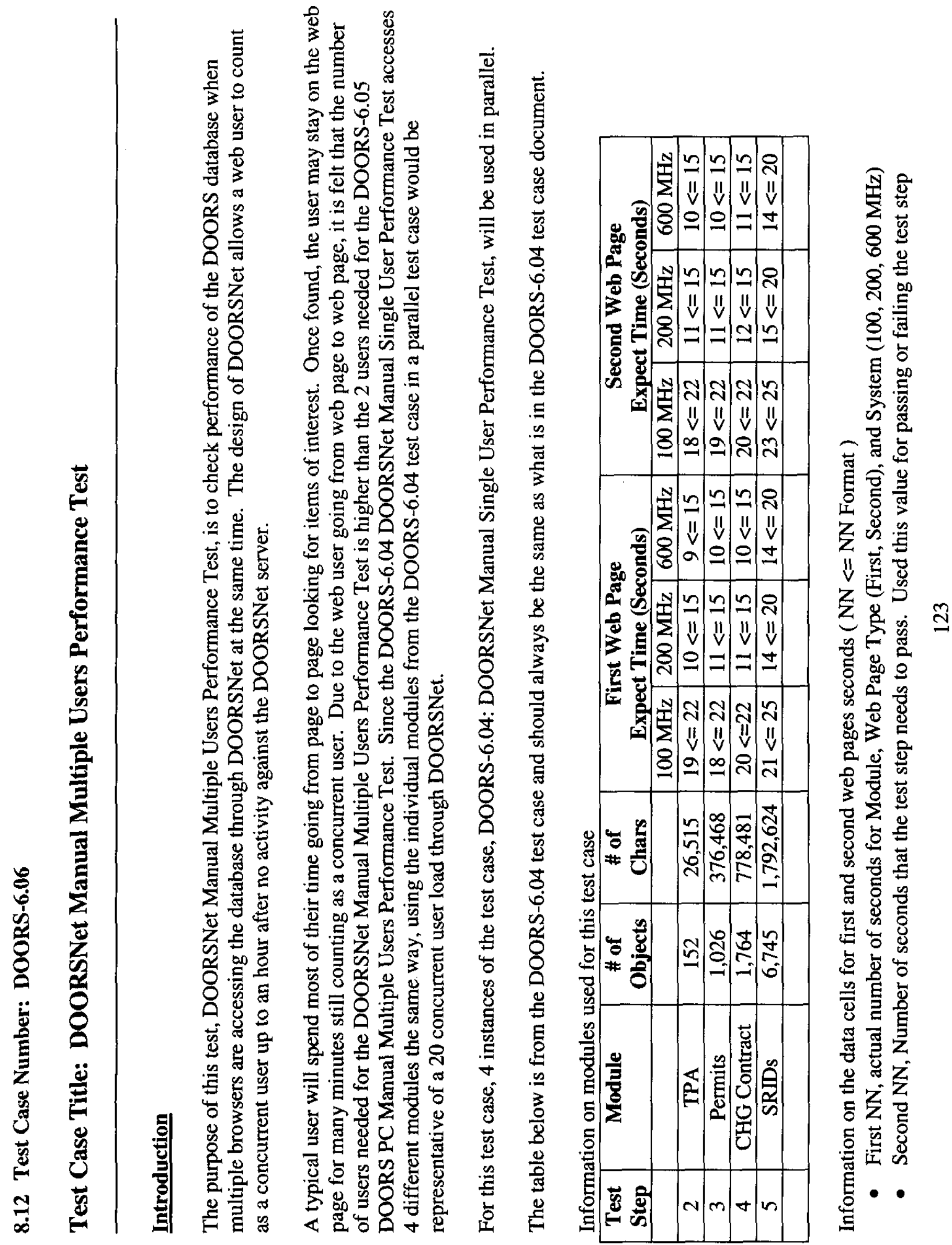



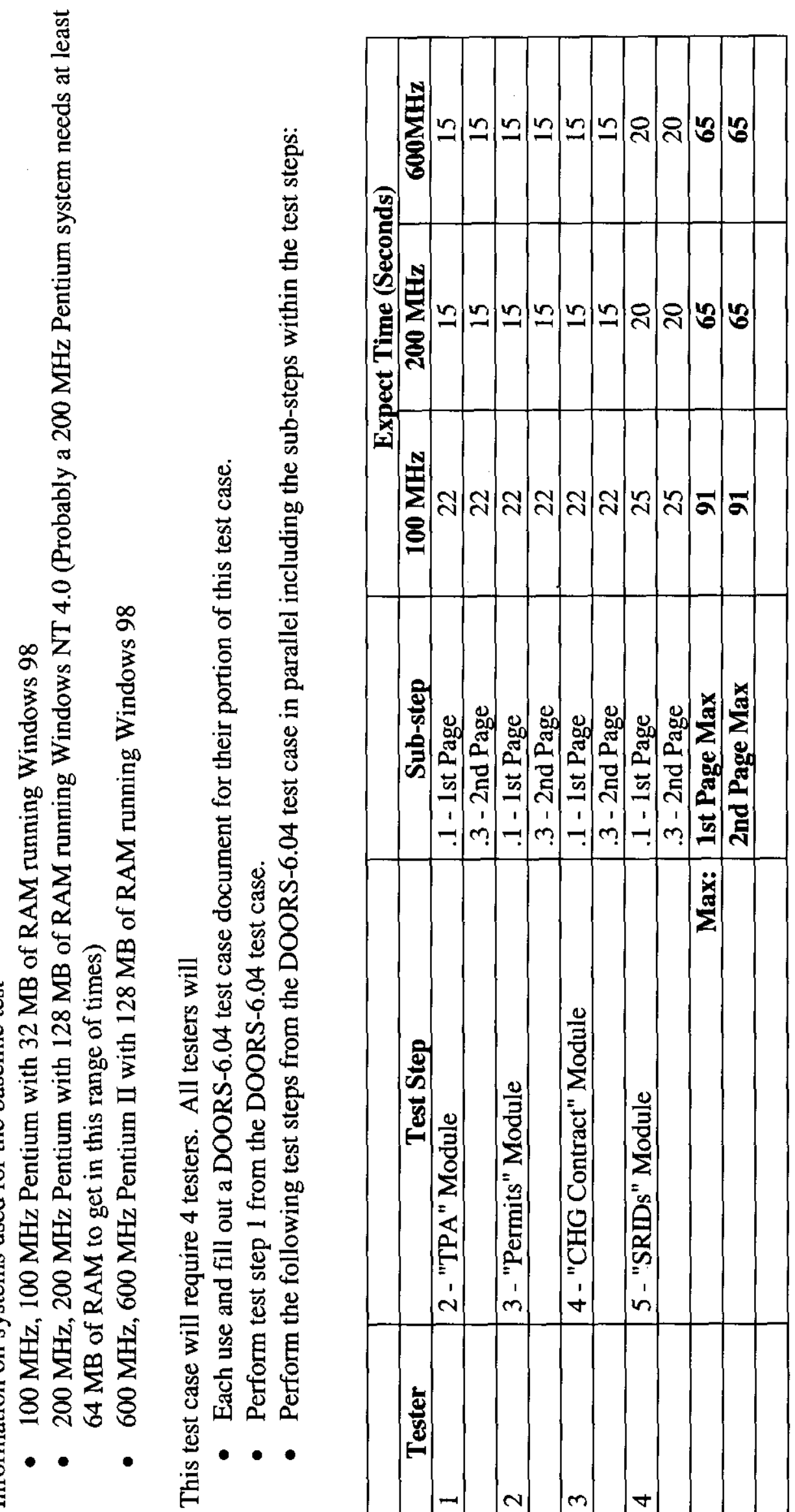

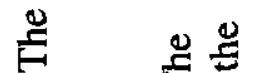

نं

充

. .5

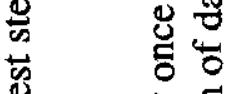

丞 究苋

5

要

क

ए

E

क 灵名

$\cong$ Ф

\% 客

品 월

品

०

总焉

需

पू

密 竎

淧

प

¿

ष

○े

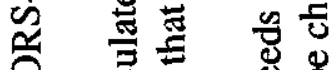

8 है 莺

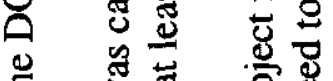

ङ 苛造

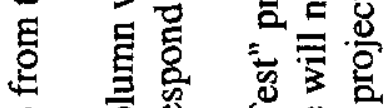

0 定

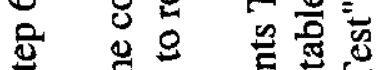

क

范

¿

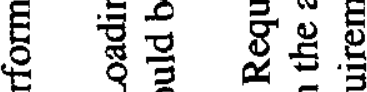

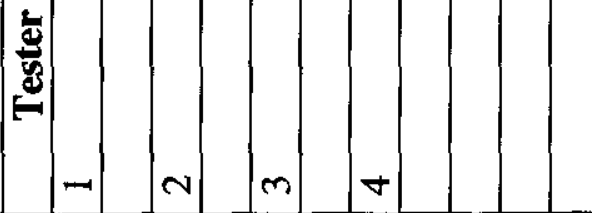

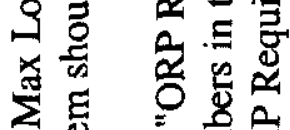

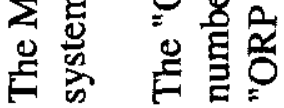


RPP-6251 REV. 0

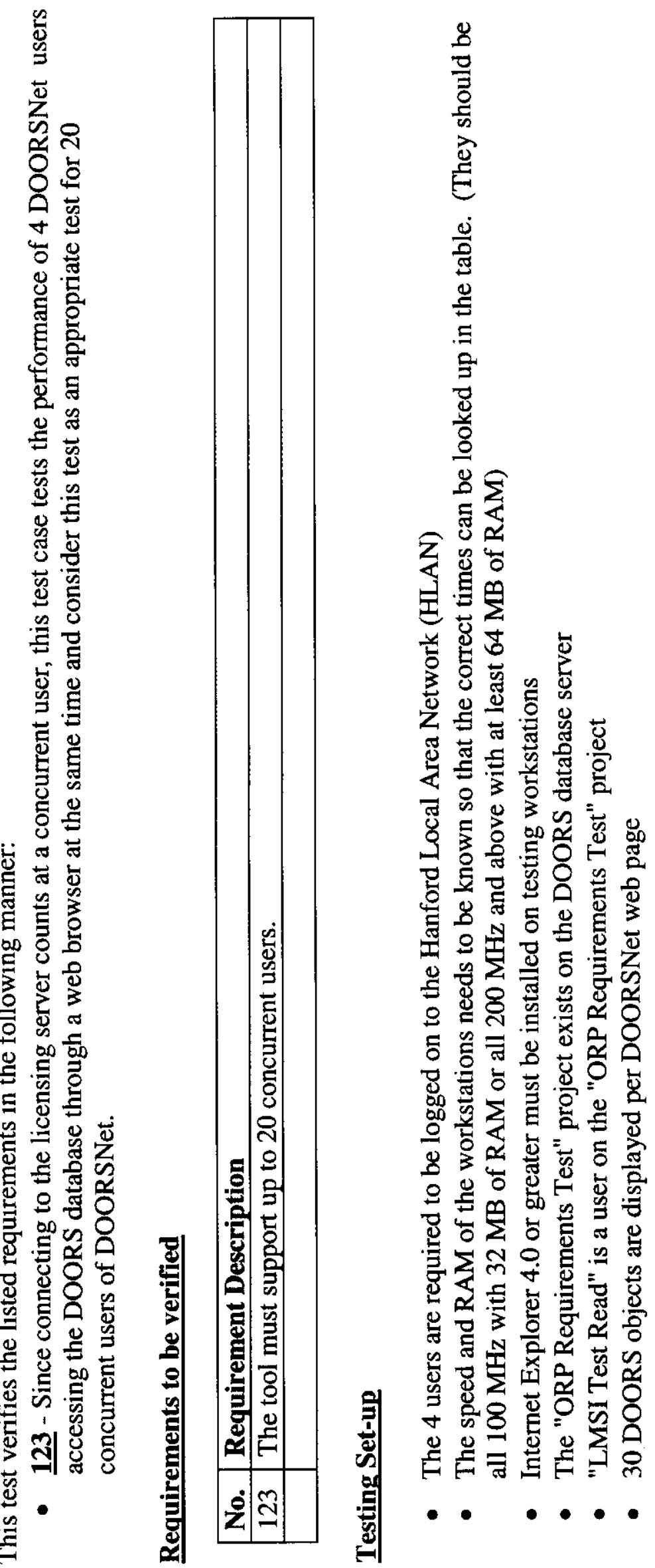

$\Xi$
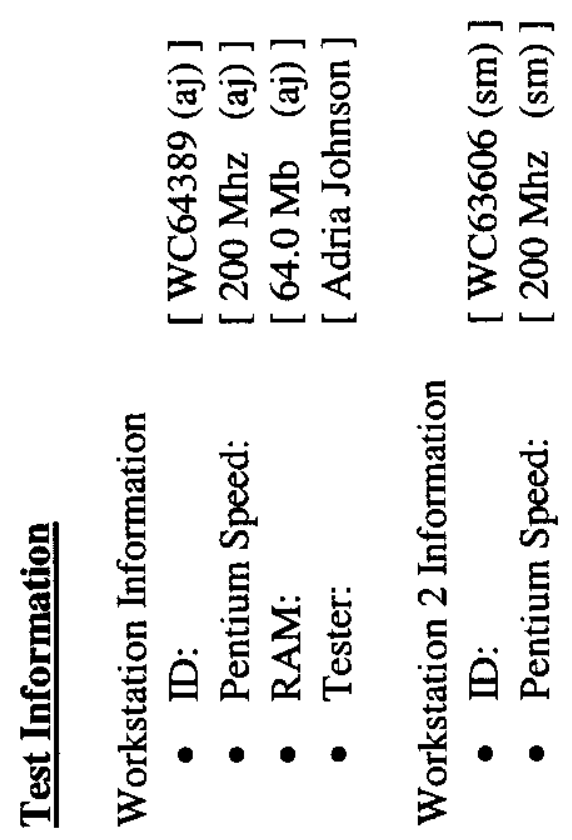

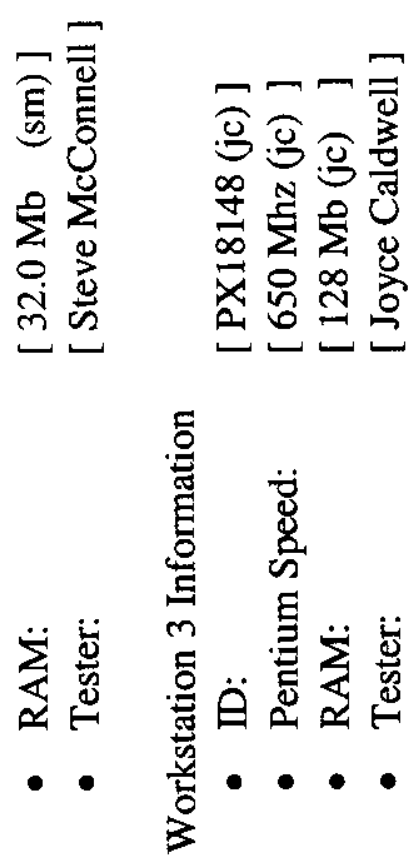
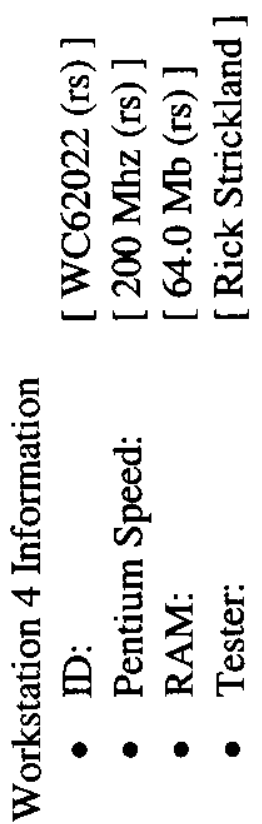

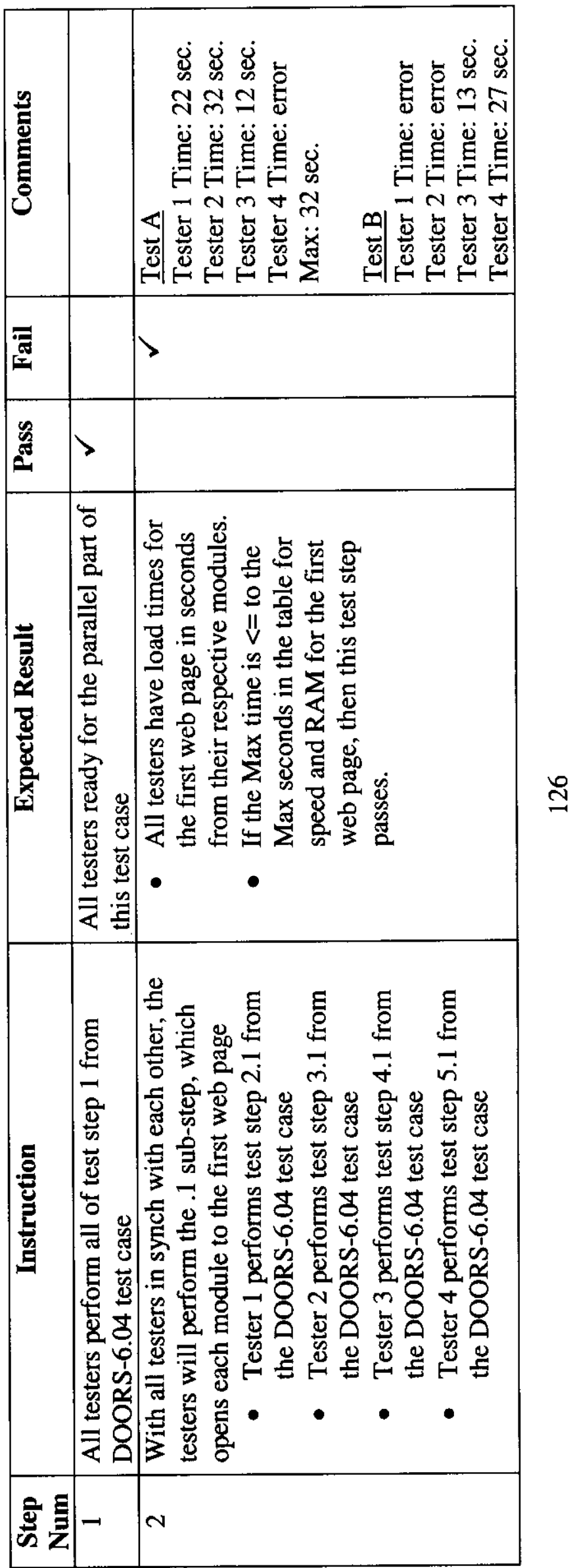


RPP-6251 REV. 0

\begin{tabular}{|c|c|c|c|}
\hline 总 & 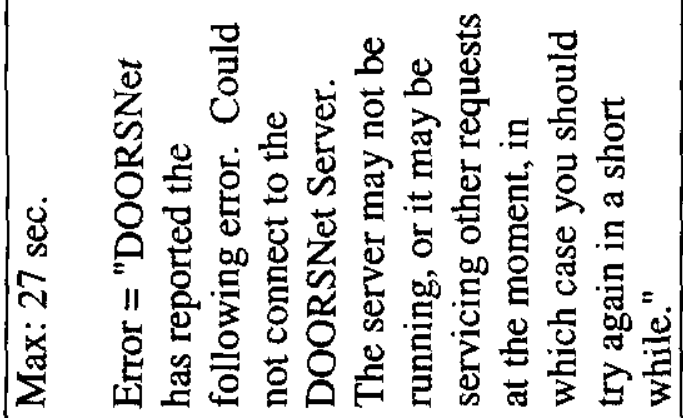 & 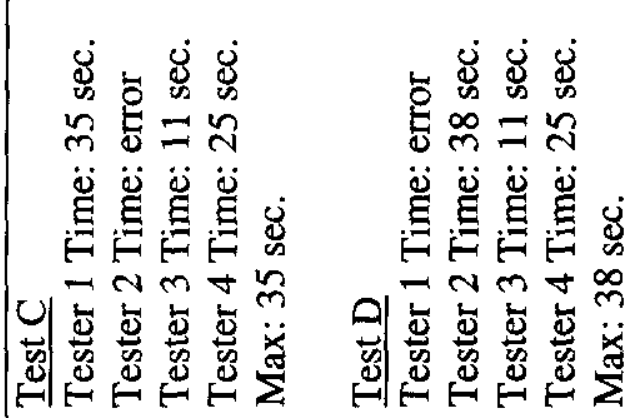 & 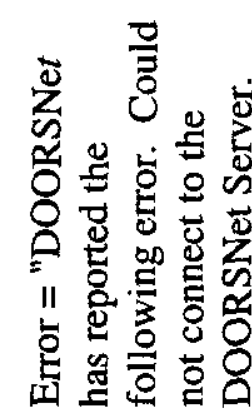 \\
\hline 疍 & & & \\
\hline 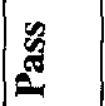 & & & \\
\hline 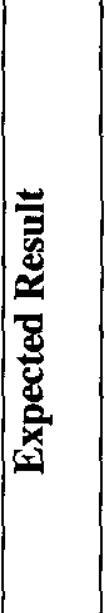 & & 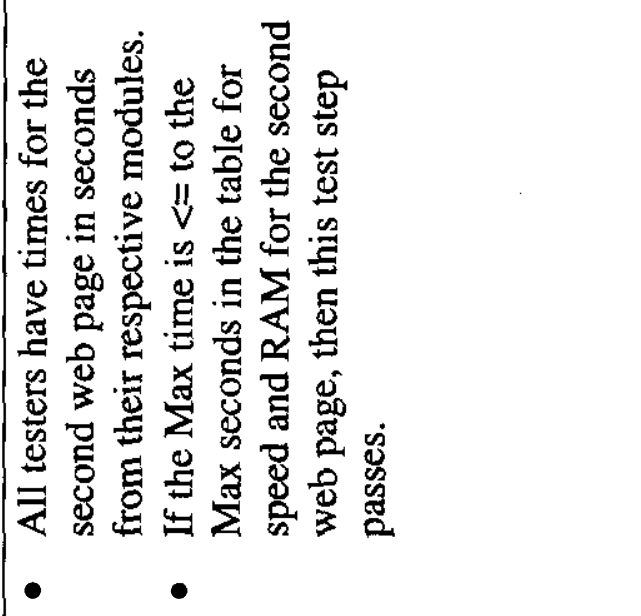 & \\
\hline 宫 & & 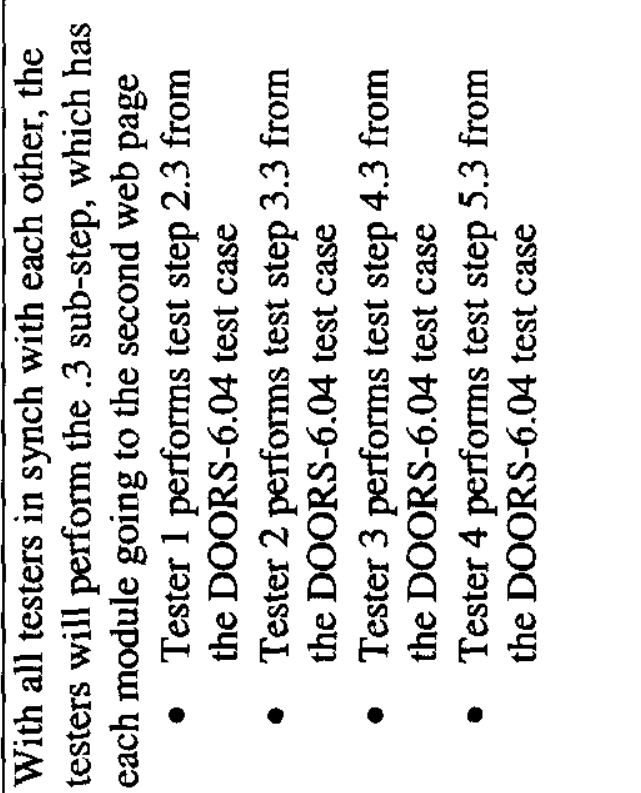 & \\
\hline 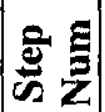 & & $m$ & \\
\hline
\end{tabular}


RPP-6251 REV. 0

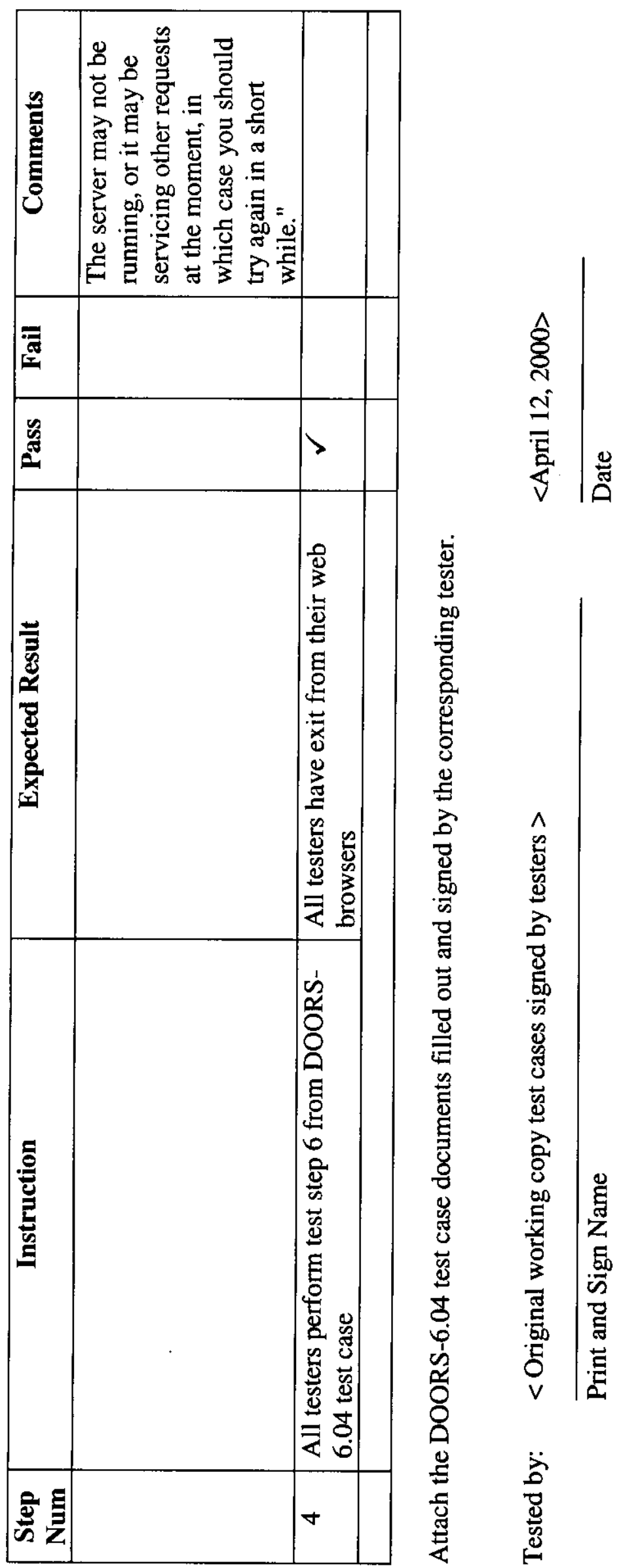




\section{Addendum 1 - Copyright Notice}

The following products are mentioned in this test plan:

- DOORS is a trademark of QSS, Limited, Mt. Arlington, New Jersey

- DOORSNet is the trademark of QSS, Limited, Mt. Arlington, New Jersey

- Excel is a trademark of Microsoft Corporation, Redmond, Washington.

- Internet Explorer is a trademark of Microsoft Corporation, Redmond, Washington.

- Outlook is a trademark of Microsoft Corporation, Redmond, Washington.

- Windows is a trademark of Microsoft Corporation, Redmond, Washington.

- Word is a trademark of Microsoft Corporation, Redmond, Washington. 